\title{
From Carbodiimides to Carbon Dioxide: Quantification of the Electrophilic Reactivities of Heteroallenes
}

\author{
Zhen Li, Robert J. Mayer, Armin R. Ofial*, and Herbert Mayr* \\ Department Chemie, Ludwig-Maximilians-Universität München \\ Butenandtstr. 5-13 (Haus F), 81377 München, Germany
}

Table of Contents

Content

page

1. General S2

2. Product Studies $\quad$ S4

3. Kinetics of the Reactions of the Heteroallenes 1 with Carbanions 2 and Enamines $3 \quad$ S19

4. NMR Kinetics of the Reactions of $1 \mathrm{~b}$ with $3 \mathrm{o}$ and 3p S28

5. Nucleophilic Reactivity of Carbanions 2 in $\mathrm{MeCN}$ S36

6. Determination of $\boldsymbol{E}$ for 1a and 1c S42

7. Procedure for Preparing $\mathrm{CO}_{2}$ Solutions in DMSO S43

$\begin{array}{ll}\text { 8. Computational Details } & \text { S47 }\end{array}$

$\begin{array}{ll}\text { 9. NMR Spectra } & \text { S75 }\end{array}$

10. References $\quad$ S106 


\section{General}

\subsection{Analytics}

${ }^{1} \mathrm{H}$ and ${ }^{13} \mathrm{C}$ NMR spectra were recorded in $\mathrm{CDCl}_{3}\left(\delta_{\mathrm{H}} 7.26, \delta_{\mathrm{C}} 77.16\right), \mathrm{CD}_{3} \mathrm{CN}\left(\delta_{\mathrm{H}} 1.94, \delta_{\mathrm{C}} 1.32\right.$, 118.26), or $d_{6}$-DMSO ( $\left.\delta_{\mathrm{H}} 2.50, \delta_{\mathrm{C}} 39.52\right)$ on 300,400 or $600 \mathrm{MHz}$ NMR spectrometers and are given in ppm. ${ }^{\mathrm{S}} 1$ The following abbreviations were used to designate chemical shift multiplicities: $\mathrm{s}=$ singlet, $\mathrm{d}=$ doublet, $\mathrm{t}=$ triplet, $\mathrm{q}=$ quartet, $\mathrm{m}=$ multiplet, $\mathrm{br}=$ broad, app $=$ apparent. The assignments of individual NMR signals were based on additional twodimensional NMR experiments (COSY, NOESY, HSQC, HMBC). HRMS and MS were recorded on a Finnigan MAT 95Q (EI) mass spectrometer or Thermo Finnigan LTQ FT Ultra (ESI). The melting points were recorded on a Büchi Melting Point B-540 device and are not corrected. Infrared (IR) spectra were recorded on a Perkin Elmer Spectrum BX-59343 instrument with a Smiths Detection DuraSampIIR Diamond ATR sensor for detection in the range $4500-600 \mathrm{~cm}^{-1}$.

\subsection{Chemicals}

DMSO (99.7\%, extra dry, over molecular sieves, AcroSeal) and acetonitrile (99.9\%, extra dry, over molecular sieves, AcroSeal) were purchased and used without further purification. Phenyl isothiocyanate 1c was purchased and then purified by vacuum distillation prior to use. Literature procedures were used to prepare $1 \mathbf{e},{ }^{\mathrm{S} 2}(\mathbf{2} \mathbf{a}-\mathbf{d})-\mathrm{H},{ }^{\mathrm{S} 3}(\mathbf{2} \mathbf{k}, \mathbf{s})-\mathrm{H}^{\mathrm{S} 4}, \mathbf{3 a}-\mathbf{d}^{\mathrm{S} 5}$ and $\mathbf{3 e}{ }^{\mathrm{S} 6}$ All other chemicals were purchased from commercial sources and used without purification.

Potassium 2-ethoxy-1-(4-cyanophenyl)-2-oxoethan-1-ide (2f-K). A solution of KHMDS (1.1 $\mathrm{g}, 5.5 \mathrm{mmol})$ in anhydrous THF $(25 \mathrm{~mL})$ was prepared. At room temperature a solution of $\mathbf{2 f -}$ H (945 mg, $5.0 \mathrm{mmol})$ in anhydrous THF $(25 \mathrm{~mL})$ was added dropwise, which caused the formation of a precipitate. The solvent was removed under vacuum and left a solid residue, which was subsequently suspended in dry ether. The suspension was filtered under argon atmosphere. The solid was washed by anhydrous diethyl ether $(5 \times 15 \mathrm{~mL})$ and then dried under vacuum to give $\mathbf{2 f - K}$ (908 $\mathrm{mg}, 80 \%$ ) as a yellow solid, which was stored in a glovebox under Ar atmosphere.

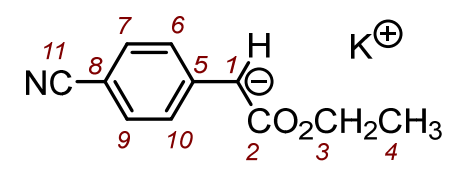


${ }^{1}$ H NMR (400 MHz, d6-DMSO): $\delta 7.82$ (d, $J=8.8 \mathrm{~Hz}, 1 \mathrm{H}, 6-\mathrm{H}$ or 10-H), $6.91(\mathrm{~d}, J=8.9 \mathrm{~Hz}$, $1 \mathrm{H}, 7-\mathrm{H}$ or 9-H), 6.81 (d, $J=8.5 \mathrm{~Hz}, 1 \mathrm{H}, 7-\mathrm{H}$ or 9-H), 6.18 (d, $J=8.5 \mathrm{~Hz}, 1 \mathrm{H}, 6-\mathrm{H}$ or 10-H), $4.01(\mathrm{~s}, 1 \mathrm{H}, 1-\mathrm{H}), 3.77$ (q, $J=7.1 \mathrm{~Hz}, 2 \mathrm{H}, 3-\mathrm{H}), 1.05$ (t, $J=7.1 \mathrm{~Hz}, 3 \mathrm{H}, 4-\mathrm{H}) .{ }^{13} \mathbf{C}$ NMR (101 MHz, $d_{6}$-DMSO): $\delta 166.5$ (C $\left.\mathrm{q}, \mathrm{C}-2\right), 151.3$ (C $\left.\mathrm{q}, \mathrm{C}-5\right), 131.1$ (C $\left.\mathrm{q}, \mathrm{C}-8\right), 130.3$ (CH, C-7 or C-9), 130.2 (CH, C-7 or C-9), 123.5 (C $\mathrm{C}, \mathrm{C}-11), 119.4$ (CH, C-6 or C-10), $117.4(\mathrm{CH}, \mathrm{C}-6$ or C-10), (88.4, unknown impurity,) $73.9(\mathrm{CH}, \mathrm{C}-1), 55.0\left(\mathrm{CH}_{2}, \mathrm{C}-3\right), 15.5\left(\mathrm{CH}_{3}, \mathrm{C}-4\right)$. HRMS (ESI $\left.{ }^{-}\right)$: $m / z$ calcd for $\left[\mathrm{C}_{11} \mathrm{H}_{10} \mathrm{NO}_{2}^{-}\right]\left(\mathbf{2 f}^{-}\right): 188.0717$, found: 188.0718. IR (ATR): $v$ 2975, 2218, 2200, $1733,1615,1543,1512,1477,1445,1416,1307,1170,1099,1059,974,890,836,742 \mathrm{~cm}^{-1}$.

Potassium 2-ethoxy-1-(4-nitrophenyl)-2-oxoethan-1-ide (2g-K). As described for the preparation of $\mathbf{2 f - K}, \mathbf{2 g}-\mathrm{K}$ was obtained from $\mathbf{2 g - H}$ (1.05 g, $5.0 \mathrm{mmol})$ and KHMDS (1.1 g, 5.5 mmol): yellow solid (988 mg, 80\%).

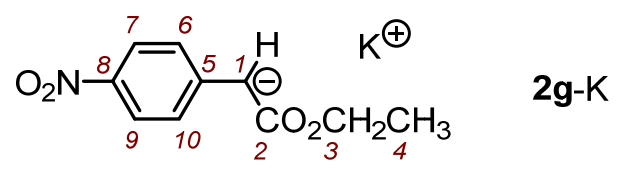

${ }^{1} \mathbf{H}$ NMR (400 MHz, d6-DMSO): $\delta 7.65(\mathrm{dd}, J=9.6 \mathrm{~Hz}, 2.0 \mathrm{~Hz}, 1 \mathrm{H}, 6-\mathrm{H}$ or 10-H), 7.47 (dd, $J$ $=9.7 \mathrm{~Hz}, 2.6 \mathrm{~Hz}, 1 \mathrm{H}, 7-\mathrm{H}$ or 9-H), $7.38(\mathrm{dd}, J=9.3 \mathrm{~Hz}, 2.5 \mathrm{~Hz}, 1 \mathrm{H}, 7-\mathrm{H}$ or 9-H), 6.14 (dd, $J$ $=9.3 \mathrm{~Hz}, 2.0 \mathrm{~Hz}, 1 \mathrm{H}, 6-\mathrm{H}$ or 10-H), $4.60(\mathrm{~s}, 1 \mathrm{H}, 1-\mathrm{H}), 3.89$ (q, $J=7.1 \mathrm{~Hz}, 2 \mathrm{H}, 3-\mathrm{H}), 1.12$ (t, $J=7.1 \mathrm{~Hz}, 3 \mathrm{H}, 4-\mathrm{H}) .{ }^{13} \mathrm{C}$ NMR (101 MHz, $d_{6}$-DMSO): $\delta 167.3\left(\mathrm{C}_{\mathrm{q}}, \mathrm{C}-2\right), 151.1\left(\mathrm{C}_{\mathrm{q}}, \mathrm{C}-5\right)$, $129.5\left(\mathrm{C}_{\mathrm{q}}, \mathrm{C}-8\right), 125.4(\mathrm{CH}, \mathrm{C}-7$ or C-9), 124.6 (CH, C-7 or C-9), $120.0(\mathrm{CH}, \mathrm{C}-6$ or C-10), $116.3\left(\mathrm{CH}, \mathrm{C}-6\right.$ or C-10), 87.1 (CH, C-1), $56.5\left(\mathrm{CH}_{2}, \mathrm{C}-3\right), 15.0\left(\mathrm{CH}_{3}, \mathrm{C}-4\right)$. HRMS (ESI $\left.{ }^{-}\right)$: $m / z$ calcd for $\left[\mathrm{C}_{10} \mathrm{H}_{10} \mathrm{NO}_{4}{ }^{-}\right]\left(\mathbf{2 g}^{-}\right): 208.0615$, found: 208.0616. IR (ATR): $v 2977,1646,1587$, $1549,1433,1352,1312,1248,1140,1093,1050,990,834,818,790,750,672 \mathrm{~cm}^{-1}$. 


\section{Product Studies}

Procedure A: To a solution of $\mathrm{KO} t \mathrm{Bu}(22 \mathrm{mg}, 0.20 \mathrm{mmol})$ in anhydrous acetonitrile $(1 \mathrm{~mL})$ at room temperature were added a solution of $2-\mathrm{H}(0.20 \mathrm{mmol})$ in anhydrous acetonitrile $(0.5 \mathrm{~mL})$ and then a solution of $1(0.20 \mathrm{mmol})$ in anhydrous acetonitrile $(0.5 \mathrm{~mL})$. After $4 \mathrm{~h}, 10 \mathrm{~mL}$ of saturated aq $\mathrm{NH}_{4} \mathrm{Cl}$ solution was added and the mixture was extracted with $\mathrm{CHCl}_{3}(3 \times 20 \mathrm{~mL})$. The organic phases were combined, washed with brine $(1 \times 30 \mathrm{~mL})$, dried with anhydrous $\mathrm{MgSO}_{4}$, and filtered. The solvent was evaporated under reduced pressure, and the residue was purified by column chromatography to give the product.

Procedure B: To a solution of $\mathbf{1}(0.20 \mathrm{mmol})$ and $\mathbf{2}-\mathrm{H}(0.20 \mathrm{mmol})$ in anhydrous $d_{6}$-DMSO $(0.6 \mathrm{~mL})$ at room temperature was added $\mathrm{NaOH}(8.0 \mathrm{mg}, 0.20 \mathrm{mmol})$. After $2 \mathrm{~h}$, the reaction mixture was analyzed by ${ }^{1} \mathrm{H}$ NMR spectroscopy without further workup.

Procedure C: To a solution of $\mathrm{KO} t \mathrm{Bu}(22 \mathrm{mg}, 0.20 \mathrm{mmol})$ in anhydrous DMSO $(1 \mathrm{~mL})$ at room temperature were added a solution of $2-\mathrm{H}(0.20 \mathrm{mmol})$ in anhydrous DMSO $(0.5 \mathrm{~mL})$ and then a solution of $1(0.20 \mathrm{mmol})$ in anhydrous DMSO $(0.5 \mathrm{~mL})$. After $4 \mathrm{~h}, 10 \mathrm{~mL}$ of aq $\mathrm{NH}_{4} \mathrm{OAc}$ solution ( $\mathrm{pH}$ ca 7) was added and the mixture was extracted with $\mathrm{CHCl}_{3}(3 \times 20 \mathrm{~mL})$. The organic phases were combined, washed with water $(2 \times 30 \mathrm{~mL})$ and brine $(1 \times 30 \mathrm{~mL})$, dried with anhydrous $\mathrm{MgSO}_{4}$, and filtered. The solvent was evaporated under reduced pressure, and the residue was purified by column chromatography to give the product. 


\subsection{Products of Reactions of Nucleophiles with PhNCO (1a)}

2-(4-Cyanophenyl)- $N$-phenyl-2-((trifluoromethyl)sulfonyl)acetamide (4ac) was obtained according to Procedure A from 1a $(24 \mathrm{mg}, 0.20 \mathrm{mmol})$ and $\mathbf{2 c}-\mathrm{H}(50 \mathrm{mg}, 0.20 \mathrm{mmol})$ : white solid (66 mg, 90\%), mp $164-168^{\circ} \mathrm{C}$.

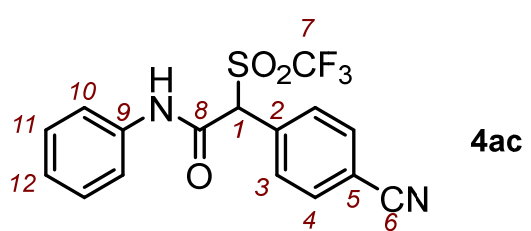

${ }^{1} \mathbf{H}$ NMR (400 MHz, CD 3 CN): $\delta 8.95$ (br s, $\left.1 \mathrm{H}, \mathrm{NH}\right), 7.93$ (d, $\left.J=8.6 \mathrm{~Hz}, 2 \mathrm{H}, 4-\mathrm{H}\right), 7.85$ (d, $J$ $=8.5 \mathrm{~Hz}, 2 \mathrm{H}, 3-\mathrm{H}), 7.52(\mathrm{~d}, J=7.5 \mathrm{~Hz}, 2 \mathrm{H}, 10-\mathrm{H}), 7.36(\mathrm{t}, J=8.0 \mathrm{~Hz}, 2 \mathrm{H}, 11-\mathrm{H}), 7.19(\mathrm{t}, J$ $=7.4 \mathrm{~Hz}, 1 \mathrm{H}, 12-\mathrm{H}), 5.79$ (s, $1 \mathrm{H}, 1-\mathrm{H}) .{ }^{13} \mathbf{C ~ N M R}\left(101 \mathrm{MHz}, \mathrm{CD}_{3} \mathrm{CN}\right): \delta 159.5$ (C-8), 138.1 (C-9), 133.8 (C-3), 132.5 (C-4), 131.7 (C-2), 130.1 (C-11), 126.5 (C-12), 121.1 (C-10), 120.7 (q, $\left.{ }^{1} J_{\mathrm{C}, \mathrm{F}}=330 \mathrm{~Hz}, \mathrm{C}-7\right), 118.9$ (C-5), 115.3 (C-6), 71.2 (C-1). HRMS (EI): $\mathrm{m} / z$ calcd for [ $\left.\mathrm{C}_{16} \mathrm{H}_{11} \mathrm{~F}_{3} \mathrm{~N}_{2} \mathrm{O}_{3} \mathrm{~S}^{++}\right]\left(\mathrm{M}^{+}\right): 368.0437$, found: 368.0447. IR (ATR): v 3308, 2959, 2239, 1665, $1600,1532,1502,1448,1367,1224,1193,1113,981,838,740,686 \mathrm{~cm}^{-1}$.

2-(4-Nitrophenyl)- $N$-phenyl-2-((trifluoromethyl)sulfonyl)acetamide (4ad) was obtained according to Procedure A from 1a (24 mg, $0.20 \mathrm{mmol})$ and $\mathbf{2 d}-\mathrm{H}$ (54 mg, $0.20 \mathrm{mmol})$ : yellow solid (66 mg, 85\%), mp $175-180^{\circ} \mathrm{C}$.

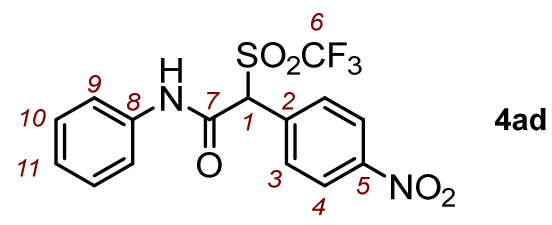

${ }^{1} \mathbf{H}$ NMR (400 MHz, CD $\left.3 \mathrm{CN}\right): \delta 8.99(\mathrm{~s}, 1 \mathrm{H}, \mathrm{NH}), 8.32(\mathrm{~d}, J=8.9 \mathrm{~Hz}, 2 \mathrm{H}, 4-\mathrm{H}), 8.02(\mathrm{~d}, J=$ $8.9 \mathrm{~Hz}, 2 \mathrm{H}, 3-\mathrm{H}), 7.54$ (d, J=7.5 Hz, $2 \mathrm{H}, 9-\mathrm{H}), 7.37$ (t, $J=8.4 \mathrm{~Hz}, 2 \mathrm{H}, 10-\mathrm{H}), 7.20$ (t, $J=$ $7.4 \mathrm{~Hz}, 1 \mathrm{H}, 11-\mathrm{H}), 5.87$ (s, $1 \mathrm{H}, 1-\mathrm{H}) .{ }^{13} \mathrm{C}$ NMR (101 MHz, CD $\left.3 \mathrm{CN}\right): \delta 159.4(\mathrm{C}-7), 150.4$ (C5), 138.1 (C-8), 133.5 (C-2), 133.0 (C-3), 130.1 (C-10), 126.5 (C-11), 124.9 (C-4), 120.7 (q, $\left.J_{\mathrm{C}, \mathrm{F}}=329 \mathrm{~Hz}, \mathrm{C}-6\right), 121.1$ (C-9), 70.9 (C-1). HRMS (EI): $\mathrm{m} / z$ calcd for $\left[\mathrm{C}_{15} \mathrm{H}_{11} \mathrm{~F}_{3} \mathrm{~N}_{2} \mathrm{O}_{5} \mathrm{~S}^{++}\right.$] $\left(\mathrm{M}^{+}\right)$: 388.0335, found: 388.0355. IR (ATR): $v$ 3318, 2954, 1664, 1601, 1524, 1494, 1448, $1367,1222,1211,1195,1110,978,870,854,740,688 \mathrm{~cm}^{-1}$. 
2-Cyano-2-(4-nitrophenyl)- $N$-phenylacetamide (4ai) was obtained according to Procedure A from $1 \mathbf{a}(24 \mathrm{mg}, 0.20 \mathrm{mmol})$ and $\mathbf{2 i}-\mathrm{H}(32 \mathrm{mg}, 0.20 \mathrm{mmol})$ : yellow solid (45 $\mathrm{mg}, 80 \%)$, mp $150-155^{\circ} \mathrm{C}$.

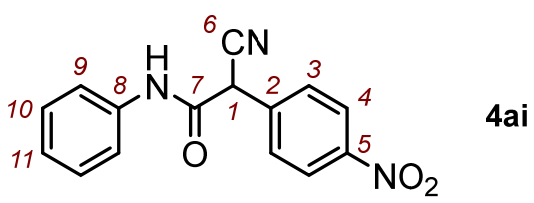

${ }^{1}$ H NMR (400 MHz, CD 3 CN): $\delta 8.81$ (br s, 1 H, NH), 8.27 (d, $\left.J=8.9 \mathrm{~Hz}, 2 \mathrm{H}, 4-\mathrm{H}\right), 7.79$ (d, $J$ $=8.8 \mathrm{~Hz}, 2 \mathrm{H}, 3-\mathrm{H}), 7.50(\mathrm{~d}, J=7.7 \mathrm{~Hz}, 2 \mathrm{H}, 9-\mathrm{H}), 7.34(\mathrm{t}, J=7.9 \mathrm{~Hz}, 2 \mathrm{H}, 10-\mathrm{H}), 7.15(\mathrm{t}, J=$ $7.4 \mathrm{~Hz}, 1 \mathrm{H}, 11-\mathrm{H}), 5.16$ (s, $1 \mathrm{H}, 1-\mathrm{H}) .{ }^{13} \mathrm{C}$ NMR (101 MHz, CD $\left.3 \mathrm{CN}\right): \delta 162.6$ (C-7), 149.3 (C5), 139.6 (C-2), 138.6 (C-8), 130.3 (C-3), 130.0 (C-10), 126.0 (C-11), 125.3 (C-4), 121.0 (C9), 117.3 (C-6), 45.7 (C-1). HRMS (EI): $m / z$ calcd for $\left[\mathrm{C}_{15} \mathrm{H}_{11} \mathrm{~N}_{3} \mathrm{O}_{3}{ }^{-+}\right]\left(\mathrm{M}^{++}\right)$: 281.0795, found: 281.0792. IR (ATR): v 3309, 2930, 2259, 1669, 1598, 1546, 1517, 1495, 1444, 1344, 1334, $1251,1110,943,878,863,844,751,730,688 \mathrm{~cm}^{-1}$.

2-(4-Nitrophenyl)- $N$-phenyl-2-(phenylsulfonyl)acetamide (4ak) was obtained according to Procedure A from 1a (24 mg, $0.20 \mathrm{mmol})$ and 2k-H (55 mg, $0.20 \mathrm{mmol})$ : pale yellow solid (71 mg, 90\%), mp 188-193 ${ }^{\circ} \mathrm{C}$.

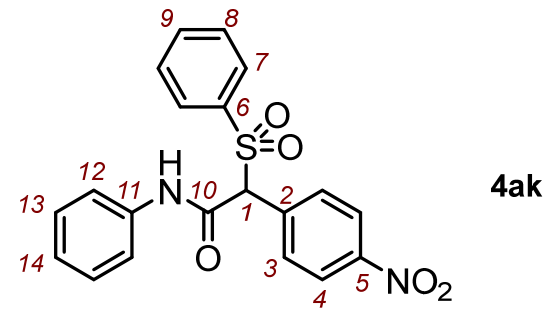

${ }^{1}$ H NMR (400 MHz, CD $\left.{ }_{3} \mathrm{CN}\right): \delta 8.75(\mathrm{~s}, 1 \mathrm{H}, \mathrm{NH}), 8.16(\mathrm{~d}, J=8.9 \mathrm{~Hz}, 2 \mathrm{H}, 4-\mathrm{H}), 7.76(\mathrm{~d}, J=$ $8.9 \mathrm{~Hz}, 2 \mathrm{H}, 3-\mathrm{H}), 7.69$ (m, 3 H, 7-H and 9-H), 7.58-7.49 (m, 2 H, 8-H), 7.46 (d, J=7.4 Hz, 2 H, 12-H), 7.41-7.29 (m, 2 H, 13-H), 7.15 (t, $J=7.4 \mathrm{~Hz}, 1 \mathrm{H}, 14-\mathrm{H}), 5.40$ (s, $1 \mathrm{H}, 1-\mathrm{H}) .{ }^{13} \mathbf{C}$ NMR (101 MHz, CD ${ }_{3} \mathrm{CN}$ ): $\delta 161.8$ (C-10), 149.6 (C-5), 138.5 (C-11), 137.7 (C-6), 136.7 (C2), 135.7 (C-9), 132.8 (C-3), 130.4 (C-7), 130.1 (C-8), 130.0 (C-13), 126.0 (C-14), 124.2 (C4), 120.9 (C-12), 75.4 (C-1). HRMS (EI): $m / z$ calcd for $\left[\mathrm{C}_{20} \mathrm{H}_{16} \mathrm{~N}_{2} \mathrm{O}_{5} \mathrm{~S}^{-+}\right]\left(\mathrm{M}^{++}\right): 396.0774$, found: 396.0773. IR (ATR): $v$ 3351, 2953, 1693, 1673, 1600, 1518, 1444, 1344, 1320, 1299, $1145,1080,975,876,847,747,726,683 \mathrm{~cm}^{-1}$. 


\subsection{Products of Reactions of Nucleophiles with TsNCO (1b)}

\section{3-Oxo-2,3-diphenyl- $N$-tosylpropanamide (5ba) via 2,3-diphenyl-3-(pyrrolidin-1-yl)-N-}

tosylacrylamide (4ba). To a solution of $1 \mathbf{b}(39 \mathrm{mg}, 0.20 \mathrm{mmol})$ in anhydrous $\mathrm{CD}_{3} \mathrm{CN}(0.5 \mathrm{~mL})$ at room temperature was added a solution of $3 \mathbf{a}(50 \mathrm{mg}, 0.20 \mathrm{mmol})$ in anhydrous $\mathrm{CD}_{3} \mathrm{CN}(0.5$ $\mathrm{mL}$ ). After $10 \mathrm{~min}$, the reaction mixture was investigated by ${ }^{1} \mathrm{H}$ NMR spectroscopy, which showed resonances that were tentatively assigned to the enamines $4 \mathbf{b a}$ (mixture of $E$-/Zisomers).

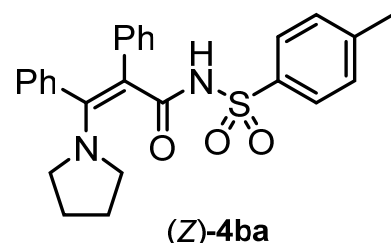

(Z)-4ba

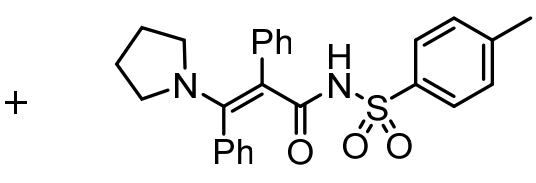

(E)-4ba

${ }^{1}$ H NMR (599 MHz, CD $\left.{ }_{3} \mathrm{CN}\right): \delta 8.03(\mathrm{~m}, 2 \mathrm{H}), 7.89(\mathrm{~d}, J=7.9 \mathrm{~Hz}, 2 \mathrm{H}), 7.59(\mathrm{~d}, J=8.0 \mathrm{~Hz}$, 2 H), 7.41 (m, 3 H), 7.38-7.29 (m, 8 H), 7.26 (m, 2 H), 7.19-7.09 (m, 6 H), 6.99 (m, 3 H), 6.86 (d, $J=7.5 \mathrm{~Hz}, 2 \mathrm{H}), 2.93$ (s, 4 H), 2.73 (s, 4 H), 2.46 (s, 3 H), 2.42 (s, 3 H), 1.78 (s, 4 H), 1.63 $(\mathrm{s}, 4 \mathrm{H})$.

After the ${ }^{1} \mathrm{H}$ NMR measurement, the 4 ba solution was hydrolyzed by adding $2 \%$ aq $\mathrm{HCl}$ (5 $\mathrm{mL})$, the mixture was extracted with $\mathrm{CHCl}_{3}(3 \times 20 \mathrm{~mL})$. The combined organic phase was washed with brine $(1 \times 30 \mathrm{~mL})$, dried with anhydrous $\mathrm{MgSO}_{4}$, and filtered. The solvent was evaporated under reduced pressure, and the residue was purified by column chromatography to give $\mathbf{5 b a}$ as pale yellow liquid (55 $\mathrm{mg}, 70 \%$ ).

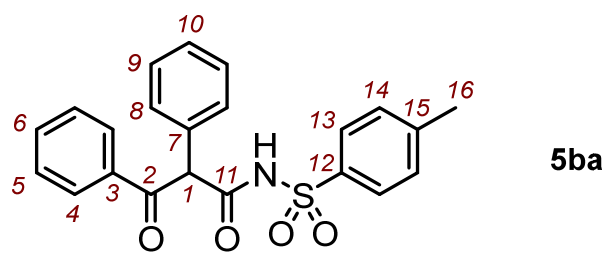

${ }^{1} \mathbf{H}$ NMR (599 MHz, d6-DMSO): $\delta 12.55$ (s, $\left.1 \mathrm{H}, \mathrm{NH}\right), 7.87$ (d, J=7.1 Hz, $\left.2 \mathrm{H}, 4-\mathrm{H}\right), 7.77$ (d, $J=8.3 \mathrm{~Hz}, 2 \mathrm{H}, 13-\mathrm{H}), 7.57$ (t, $J=7.4 \mathrm{~Hz}, 1 \mathrm{H}, 6-\mathrm{H}), 7.46-7.40$ (m, $4 \mathrm{H}, 5-\mathrm{H}$ and 14-H), 7.29$7.22(\mathrm{~m}, 5 \mathrm{H}, 8-\mathrm{H}, 9-\mathrm{H}$, and 10-H), 5.93 (s, $1 \mathrm{H}, 1-\mathrm{H}), 2.40$ (s, $3 \mathrm{H}, 16-\mathrm{H}) .{ }^{13} \mathbf{C}$ NMR $(151 \mathrm{MHz}$, $d_{6}$-DMSO): $\delta 193.5$ (C-2), 166.8 (C-11), 144.2 (C-15), 136.2 (C-12), 135.2 (C-3), 133.5 (C-6), 132.6 (C-7), 129.8 (C-8), 129.5 (C-14), 128.69 (C-5), 128.67 (C-4), 128.4 (C-9), 127.8 (C-10), 127.5 (C-13), 60.3 (C-1), 21.1 (C-16). HRMS (ESI $\left.{ }^{-}\right): m / z$ calcd for $\left[\mathrm{C}_{22} \mathrm{H}_{18} \mathrm{NO}_{4} \mathrm{~S}^{-}\right]\left(\mathrm{M}-\mathrm{H}^{+}\right)$: 392.0962, found: 392.0965. IR (ATR): $v$ 3236, 2925, 1717, 1678, 1596, 1448, 1341, 1169, $1085,889,830,813,761,698,658 \mathrm{~cm}^{-1}$. 
3,5-Dimethyl- $\boldsymbol{N}$-tosyl-1H-pyrrole-2-carboxamide (4bf). To a solution of $\mathbf{1 b}$ (59 $\mathrm{mg}, 0.30$ $\mathrm{mmol})$ in anhydrous acetonitrile $(1.0 \mathrm{~mL})$ at room temperature was added a solution of $\mathbf{3 f}$ (29 $\mathrm{mg}, 0.30 \mathrm{mmol})$ in anhydrous acetonitrile $(0.5 \mathrm{~mL})$. After $1 \mathrm{~min}$, the solvent was removed and the residue was purified by column chromatography to furnish $\mathbf{4 b f}$ as a white solid (79 $\mathrm{mg}$, $90 \%)$, mp $134-138^{\circ} \mathrm{C}$.

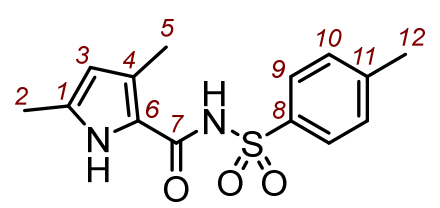

$4 b f$

${ }^{1}$ H NMR (400 MHz, CD $3 \mathrm{CN}$ ): $\delta 9.50$ (br s, $\left.1 \mathrm{H}, \mathrm{NH}\right), 8.82$ (br s, $\left.1 \mathrm{H}, \mathrm{NH}\right), 7.91$ (d, $J=8.4 \mathrm{~Hz}$, $2 \mathrm{H}, 9-\mathrm{H}), 7.37$ (d, J=7.8 Hz, 2 H, 10-H), 5.79 (m, 1 H, 3-H), 2.41 (s, $3 \mathrm{H}, 12-\mathrm{H}), 2.24$ (s, 3 H, 5-H), 2.17 (s, 3 H, 2-H). ${ }^{13}$ C NMR (101 MHz, CD $3 \mathrm{CN}$ ): $\delta 158.5$ (C-7), 145.8 (C-11), 138.1 (C8), 135.6 (C-1), 130.4 (C-10), 130.1 (C-4), 129.0 (C-9), 119.5 (C-6), 113.0 (C-3), 21.6 (C-12), 13.2 (C-5), 12.9 (C-2). HRMS (EI): $m / z$ calcd for $\left[\mathrm{C}_{14} \mathrm{H}_{16} \mathrm{~N}_{2} \mathrm{O}_{3} \mathrm{~S}^{++}\right]\left(\mathrm{M}^{+}\right)$: 292.0876, found: 292.0875. IR (ATR) v 3281, 1613, 1467, 1305, 1164, 1056, 968, 884, 802, 756, $660 \mathrm{~cm}^{-1}$.

\section{(E)-5-(4-(Dimethylamino)benzylidene)- $\mathrm{N}$-tosylcyclopenta-1,3-diene-1-carboxamide (4bh).}

To a solution of $\mathbf{1 b}(59 \mathrm{mg}, 0.30 \mathrm{mmol})$ in anhydrous acetonitrile $(1.0 \mathrm{~mL})$ at room temperature was added a solution of $\mathbf{3 h}(59 \mathrm{mg}, 0.30 \mathrm{mmol})$ in anhydrous acetonitrile $(0.5 \mathrm{~mL})$. After 11 $\mathrm{min}$, the solvent was removed, and the residue was purified by column chromatography to give a 4:1-mixture of $\mathbf{4 b h}$ and an unknown product: red liquid (106 $\mathrm{mg}$ ). Only the resonances of the major product $\mathbf{4 b h}$ (approx. $70 \%$ yield) are listed below.

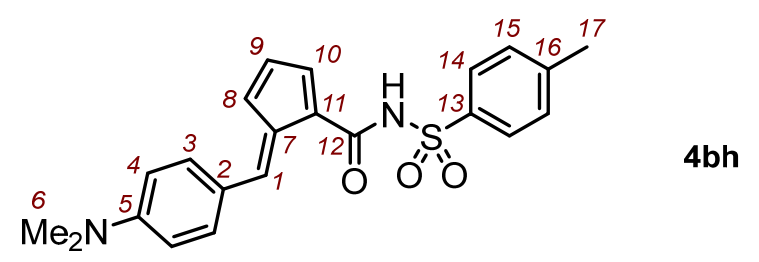

${ }^{1} \mathbf{H}$ NMR (400 MHz, $\left.\mathrm{CDCl}_{3}\right): \delta 8.92(\mathrm{~s}, 1 \mathrm{H}, \mathrm{NH}), 8.35(\mathrm{~s}, 1 \mathrm{H}, 1-\mathrm{H}), 8.03(\mathrm{~d}, J=8.1 \mathrm{~Hz}, 2 \mathrm{H}$, 14-H), 7.55 (d, $J=8.7 \mathrm{~Hz}, 2 \mathrm{H}, 3-\mathrm{H}), 7.32$ (d, $J=8.0 \mathrm{~Hz}, 2 \mathrm{H}, 15-\mathrm{H}), 7.13$ (s, $1 \mathrm{H}, 10-\mathrm{H}), 7.08$ (d, $J=5.3 \mathrm{~Hz}, 1 \mathrm{H}, 8-\mathrm{H}), 6.65$ (d, $J=8.7 \mathrm{~Hz}, 2 \mathrm{H}, 4-\mathrm{H}), 6.48-6.46$ (m, $1 \mathrm{H}, 9-\mathrm{H}), 3.04$ (s, $6 \mathrm{H}$, 6-H), 2.40 (s, 3 H, 17-H). ${ }^{13}$ C NMR (101 MHz, $\left.\mathrm{CDCl}_{3}\right): \delta 161.7$ (C-12), 152.2 (C-5), 147.7 (C1), 144.6 (C-16), 136.4 (C-13), 135.7 (C-7), 135.2 (C-10), 134.6 (C-3), 129.6 (C-15), 128.7 (C9 and C-8), 128.4 (C-14), 125.1 (C-11), 124.3 (C-2), 111.9 (C-4), 40.1 (C-6), 21.7 (C-17). HRMS (ESI $\left.{ }^{-}\right): m / z$ calcd for $\left[\mathrm{C}_{22} \mathrm{H}_{21} \mathrm{~N}_{2} \mathrm{O}_{3} \mathrm{~S}^{-}\right]\left(\mathrm{M}-\mathrm{H}^{+}\right): 393.1278$, found: 393.1280. 
1-Methyl- $\boldsymbol{N}$-tosyl-1H-pyrrole-2-carboxamide (4bj). To a solution of $\mathbf{1 b}$ (59 $\mathrm{mg}, 0.30 \mathrm{mmol}$ ) in anhydrous dichloromethane $(1.0 \mathrm{~mL})$ at room temperature was added a solution of $\mathbf{3 j} \mathbf{j}(24 \mathrm{mg}$, $0.30 \mathrm{mmol})$ in anhydrous dichloromethane $(0.5 \mathrm{~mL})$. After $9.5 \mathrm{~h}$, the solvent was evaporated, and the residue was purified by column chromatography to furnish $\mathbf{4 b j}$ as a white solid $(78 \mathrm{mg}$, 93\%), mp $168-172{ }^{\circ} \mathrm{C}$ (lit.: $\mathrm{mp} 167-169^{\circ} \mathrm{C}^{\mathrm{S} 7}$ ).

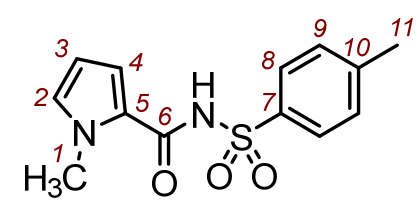

4bj

${ }^{1} \mathbf{H}$ NMR (400 MHz, CDCl 3$): \delta 9.13(\mathrm{~s}, 1 \mathrm{H}, \mathrm{NH}), 8.01$ (d, $\left.J=8.4 \mathrm{~Hz}, 2 \mathrm{H}, 8-\mathrm{H}\right), 7.33$ (d, $J=$ $7.9 \mathrm{~Hz}, 2 \mathrm{H}, 9-\mathrm{H}), 6.91$ (dd, $J=4.2 \mathrm{~Hz}, 1.7 \mathrm{~Hz}, 1 \mathrm{H}, 4-\mathrm{H}), 6.77$ (t, $J=2.1 \mathrm{~Hz}, 1 \mathrm{H}, 2-\mathrm{H}), 6.06$ (dd, $J=4.2,2.5 \mathrm{~Hz}, 1 \mathrm{H}, 3-\mathrm{H}), 3.82$ (s, $3 \mathrm{H}, 1-\mathrm{H}), 2.43$ (s, $3 \mathrm{H}, 11-\mathrm{H}) .{ }^{13} \mathbf{C}$ NMR $(101 \mathrm{MHz}$, $\mathrm{CDCl}_{3}$ ): $\delta 157.8$ (C-6), 144.9 (C-10), 136.3 (C-7), 131.3 (C-2), 129.7 (C-9), 128.4 (C-8), 122.4 (C-5), 116.4 (C-4), 108.5 (C-3), 37.2 (C-1), 21.8 (C-11). HRMS (EI): m/z calcd for $\left[\mathrm{C}_{13} \mathrm{H}_{14} \mathrm{~N}_{2} \mathrm{O}_{3} \mathrm{~S}^{++}\right]\left(\mathrm{M}^{+}\right): 278.0720$, found: 278.0722. IR (ATR): $v 3287,1673,1594,1531,1413$, $1342,1160,1069,1061,1010,875,813,733,657 \mathrm{~cm}^{-1}$.

4-Ethoxy-1-tosylazetidin-2-one (4bo). To a solution of $\mathbf{1 b}(99 \mathbf{m g}, 0.50 \mathrm{mmol}$ ) in anhydrous dichloromethane $(1.0 \mathrm{~mL})$ at room temperature was added a solution of $\mathbf{3 0}(36 \mathrm{mg}, 0.50 \mathrm{mmol})$ in anhydrous dichloromethane $(1.0 \mathrm{~mL})$. After $2 \mathrm{~h}$, the solvent was evaporated, and the residue was recrystallized from $\mathrm{Et}_{2} \mathrm{O}$ to give 4 bo as a white solid (128 mg, 95\%), mp $77-82^{\circ} \mathrm{C}$ (lit.: $\left.\operatorname{mp} 56-63{ }^{\circ} \mathrm{C}^{\mathrm{S} 8}\right)$.

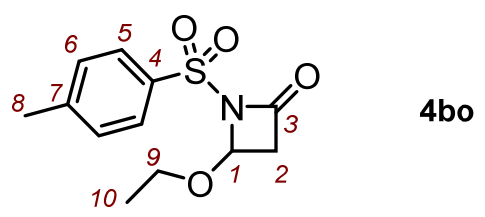

${ }^{1} \mathbf{H}$ NMR (400 MHz, $\left.\mathrm{CD}_{2} \mathrm{Cl}_{2}\right): \delta 7.85(\mathrm{~d}, J=8.5 \mathrm{~Hz}, 2 \mathrm{H}, 5-\mathrm{H}), 7.39(\mathrm{~d}, J=8.1 \mathrm{~Hz}, 2 \mathrm{H}, 6-\mathrm{H})$, $5.50(\mathrm{dd}, J=4.9 \mathrm{~Hz}, 2.2 \mathrm{~Hz}, 1 \mathrm{H}, 1-\mathrm{H}), 3.96$ (dq, $J=9.4 \mathrm{~Hz}, 7.1 \mathrm{~Hz}, 1 \mathrm{H}, 9-\mathrm{H}), 3.70$ (dq, $J=$ 9.4, 7.0 Hz, 1 H, 9-H), 3.19 (dd, $J=16.2 \mathrm{~Hz}, 4.9 \mathrm{~Hz}, 1 \mathrm{H}, 2-\mathrm{H}), 2.89$ (dd, $J=16.2 \mathrm{~Hz}, 2.2 \mathrm{~Hz}$, $1 \mathrm{H}, 2-\mathrm{H}), 2.45$ (s, $3 \mathrm{H}, 8-\mathrm{H}), 1.20$ (t, $J=7.1 \mathrm{~Hz}, 3 \mathrm{H}, 10-\mathrm{H}) .{ }^{13} \mathbf{C} \mathbf{N M R}\left(101 \mathrm{MHz}, \mathrm{CD}_{2} \mathrm{Cl}_{2}\right): \delta$ 163.3 (C-3), 146.1 (C-7), 136.7 (C-4), 130.5 (C-6), 127.8 (C-5), 84.8 (C-1), 67.1 (C-9), 45.3 (C-2), $22.0(\mathrm{C}-8), 15.4(\mathrm{C}-10)$. HRMS $\left(\mathrm{ESI}^{+}\right): \mathrm{m} / z$ calcd for $\left[\mathrm{C}_{12} \mathrm{H}_{16} \mathrm{NO}_{4} \mathrm{~S}^{+}\right]\left(\mathrm{M}+\mathrm{H}^{+}\right): 270.0795$, found: 270.0793. IR (ATR): $v$ 3272, 2978, 1795, 1723, 1597, 1443, 1354, 1273, 1163, 1113 , $1083,961,813,705,674 \mathrm{~cm}^{-1}$. 
3,3-Dimethyl-1-tosyl-4-(trimethylsiloxy)azetidin-2-one (4bp). To a solution of $\mathbf{1 b}$ (99 mg, $0.50 \mathrm{mmol})$ in anhydrous dichloromethane $(1.0 \mathrm{~mL})$ at room temperature was added a solution of $3 \mathbf{p}$ (72 $\mathrm{mg}, 0.50 \mathrm{mmol})$ in anhydrous dichloromethane $(1.0 \mathrm{~mL})$. After $6 \mathrm{~h}$, the solvent was evaporated, and the residue was recrystallized from $\mathrm{Et}_{2} \mathrm{O}$ to give $4 \mathbf{b p}$ as a white solid (153 $\mathrm{mg}$, 90\%), mp 89-93 ${ }^{\circ} \mathrm{C}$.

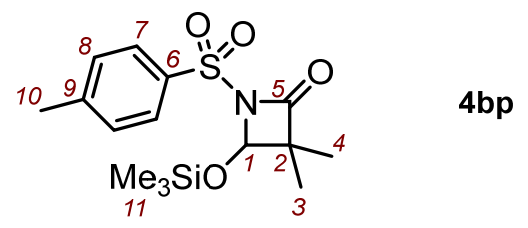

${ }^{1} \mathbf{H}$ NMR (400 MHz, $\left.\mathrm{CD}_{2} \mathrm{Cl}_{2}\right): \delta 7.82(\mathrm{~d}, J=8.4 \mathrm{~Hz}, 2 \mathrm{H}, 7-\mathrm{H}), 7.37(\mathrm{~d}, J=7.9 \mathrm{~Hz}, 2 \mathrm{H}, 8-\mathrm{H})$, 5.29 (s, 1 H, 1-H), 2.45 (s, 3 H, 10-H), 1.17 (s, 3 H, 3-H or 4-H), 1.08 (s, 3 H, 3-H or 4-H), 0.22 (s, 9 H, 11-H). ${ }^{13}$ C NMR (101 MHz, $\mathrm{CD}_{2} \mathrm{Cl}_{2}$ ): $\delta 170.2$ (C-5), 145.8 (C-9), 137.1 (C-6), 130.4 (C-8), 127.7 (C-7), 86.7 (C-1), 56.5 (C-2), 22.0 (C-10), 20.0 (C-3 or C-4), 16.3 (C-3 or C-4), 0.0 (C-11). HRMS $\left(\mathrm{ESI}^{+}\right): m / z$ calcd for $\left[\mathrm{C}_{15} \mathrm{H}_{27} \mathrm{~N}_{2} \mathrm{O}_{4} \mathrm{SSi}^{+}\right]\left(\mathrm{M}+\mathrm{NH}_{4}^{+}\right): 359.1455$, found: 359.1457. IR (ATR): v 3357, 3260, 2955, 1785, 1596, 1463, 1403, 1358, 1295, 1254, 1189 , $1165,1139,1116,1083,1041,873,847,814,746,666 \mathrm{~cm}^{-1}$.

\subsection{Products of Reactions of Nucleophiles with PhNCS (1c)}

Sodium 2,2-dicyano-1-(phenylamino)ethene-1-thiolate $\left(4 \mathrm{cn}^{-} \mathrm{Na}^{+}\right)$was obtained according to Procedure B from 1c (24 mg, $0.20 \mathrm{mmol})$ and $\mathbf{2 n}-\mathrm{H}(13 \mathrm{mg}, 0.20 \mathrm{mmol})$.<smiles>N#CC(C#N)=C([O-])Nc1ccccc1</smiles>

\section{$4 \mathrm{cn}^{-} \mathrm{Na}^{+}$}

${ }^{1}$ H NMR (400 MHz, d6-DMSO): $\delta 8.89$ (s, $\left.1 \mathrm{H}\right), 7.49$ (d, $J=7.4$ Hz, $\left.2 \mathrm{H}\right), 7.24-7.20$ (m, $2 \mathrm{H}$ ), $7.01(\mathrm{t}, J=7.4 \mathrm{~Hz}, 1 \mathrm{H})$. HRMS $\left(\mathrm{ESI}^{-}\right): \mathrm{m} / z$ calcd for $\left[\mathrm{C}_{10} \mathrm{H}_{6} \mathrm{~N}_{3} \mathrm{~S}^{-}\right]\left(\mathbf{4} \mathbf{c n}^{-}\right): 200.0288$, found: 200.0288 .

Sodium (Z)-2-(ethoxycarbonyl)-3-oxo-1-(phenylamino)but-1-ene-1-thiolate $\left(4 \mathrm{co}^{-} \mathrm{Na}^{+}\right)$was obtained according to Procedure B from $1 \mathrm{c}(24 \mathrm{mg}, 0.20 \mathrm{mmol})$ and $2 \mathrm{o}-\mathrm{H}$ (26 mg, $0.20 \mathrm{mmol})$.

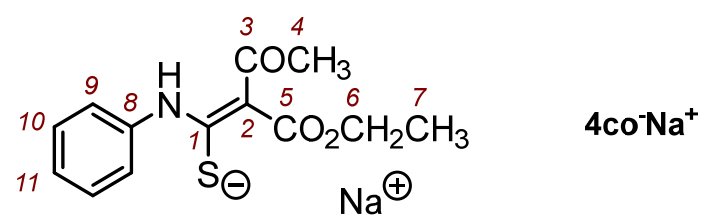

${ }^{1} \mathbf{H}$ NMR (400 MHz, d6-DMSO): $\delta 14.44$ (s, $\left.1 \mathrm{H}, \mathrm{NH}\right), 7.79$ (d, $\left.J=7.4 \mathrm{~Hz}, 2 \mathrm{H}, 9-\mathrm{H}\right), 7.22$ (t, $J=8.4 \mathrm{~Hz}, 2 \mathrm{H}, 10-\mathrm{H}), 6.96(\mathrm{t}, J=7.3 \mathrm{~Hz}, 1 \mathrm{H}, 11-\mathrm{H}), 4.02(\mathrm{q}, J=7.1 \mathrm{~Hz}, 2 \mathrm{H}, 6-\mathrm{H}), 1.77$ (s, $3 \mathrm{H}, 4-\mathrm{H}), 1.21$ (t, $J=7.1 \mathrm{~Hz}, 3 \mathrm{H}, 7-\mathrm{H}) .{ }^{13} \mathbf{C}$ NMR (101 MHz, $d_{6}$-DMSO): $\delta 184.4$ (C-1), 178.6 
(C-3), 172.1 (C-5), 142.0 (C-8), 127.9 (C-10), 122.3 (C-11), 122.1 (C-9), 112.3 (C-2), 59.2 (C6), 26.5 (C-4), 14.1 (C-7). HRMS (ESI $\left.{ }^{-}\right): m / z$ calcd for $\left[\mathrm{C}_{13} \mathrm{H}_{14} \mathrm{NO}_{3} \mathrm{~S}^{-}\right]\left(\mathbf{4} \mathbf{c o}^{-}\right): 264.0700$, found: 264.0701 .

Sodium 2-acetyl-3-oxo-1-(phenylamino)but-1-ene-1-thiolate $\left(4 \mathrm{cp}^{-} \mathrm{Na}^{+}\right)$was obtained according to Procedure B from 1c (24 mg, $0.20 \mathrm{mmol})$ and 2p-H (20 mg, $0.20 \mathrm{mmol})$.

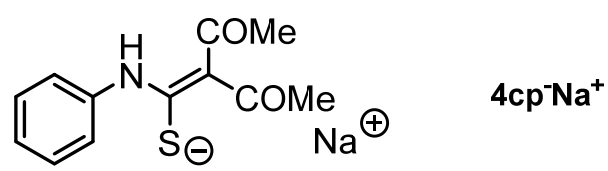

${ }^{1}$ H NMR (400 MHz, $d_{6}$-DMSO): $\delta 14.60$ (s, $\left.1 \mathrm{H}\right), 7.77$ (d, $\left.J=7.3 \mathrm{~Hz}, 2 \mathrm{H}\right), 7.25-7.21$ (m, 2 $\mathrm{H}), 6.97(\mathrm{t}, J=7.3 \mathrm{~Hz}, 1 \mathrm{H}), 2.11(\mathrm{~s}, 6 \mathrm{H})$. HRMS $\left(\mathrm{ESI}^{-}\right): \mathrm{m} / z$ calcd for $\left[\mathrm{C}_{12} \mathrm{H}_{12} \mathrm{NO}_{2} \mathrm{~S}^{-}\right]\left(\mathbf{4} \mathbf{c p}^{-}\right)$: 234.0594, found: 234.0595 .

2-((Methylthio)(phenylamino)methylene)malononitrile (6cn): To a solution of $1 \mathrm{c}$ (54 mg, $0.40 \mathrm{mmol})$ and $\mathbf{2 n}-\mathrm{H}(27 \mathrm{mg}, 0.41 \mathrm{mmol})$ in anhydrous DMSO $(3 \mathrm{~mL})$ at room temperature was added $\mathrm{NaOH}(16 \mathrm{mg}, 0.40 \mathrm{mmol})$. After $2 \mathrm{~h}$, a solution of iodomethane $(57 \mathrm{mg}, 0.40 \mathrm{mmol})$ in anhydrous DMSO (1 mL) was added at room temperature. After $2 \mathrm{~h}, 20 \mathrm{~mL}$ of saturated $\mathrm{NH}_{4} \mathrm{Cl}$ solution was added and the mixture was extracted with EtOAc $(3 \times 20 \mathrm{~mL})$. The combined organic phase was washed with water $(2 \times 30 \mathrm{~mL})$ and brine $(1 \times 30 \mathrm{~mL})$, dried with anhydrous $\mathrm{MgSO}_{4}$, and filtered. The solvent was evaporated under reduced pressure, and the residue was purified by column chromatography to give $6 \mathbf{c n}$ as a white solid (69 $\mathrm{mg}, 80 \%$ ), $\mathrm{mp}$ 173-178 ${ }^{\circ} \mathrm{C}$ (lit.: mp $169{ }^{\circ} \mathrm{C}$ from ethanol ${ }^{\mathrm{S} 9}$ ).

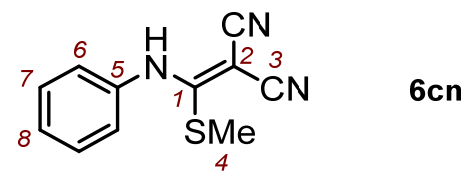

${ }^{1}$ H NMR (400 MHz, d6-DMSO): $\delta 10.54$ (s, $\left.1 \mathrm{H}, \mathrm{NH}\right), 7.42$ (t, $\left.J=7.7 \mathrm{~Hz}, 2 \mathrm{H}, 7-\mathrm{H}\right), 7.30$ (d, $J=8.4 \mathrm{~Hz}, 2 \mathrm{H}, 6-\mathrm{H}), 7.27$ (t, $J=7.2 \mathrm{~Hz}, 1 \mathrm{H}, 8-\mathrm{H}), 2.52$ (s, $3 \mathrm{H}, 4-\mathrm{H}) .{ }^{13} \mathbf{C}$ NMR $(101 \mathrm{MHz}$, $d_{6 \text {-DMSO): } \delta} 171.5$ (C-1), 138.3 (C-5), 129.2 (C-7), 126.6 (C-8), 123.9 (C-6), 52.9 (C-2), 15.8 (C-4) (C-3 is missing). HRMS (EI): $m / z$ calcd for $\left[\mathrm{C}_{11} \mathrm{H}_{9} \mathrm{~N}_{3} \mathrm{~S}^{++}\right]\left(\mathrm{M}^{++}\right)$: 215.0512, found: 215.0504. IR (ATR): v 3285, 2194, 2179, 1594, 1520, 1489, 1448, 1425, 1262, 965, 757, 699 $\mathrm{cm}^{-1}$. 


\subsection{Products of Reactions of Nucleophiles with $p$-Nitrophenylisothiocyanate (1d)}

Sodium 2,2-dicyano-1-((4-nitrophenyl)amino)ethene-1-thiolate $\left(4 \mathrm{dn}^{-} \mathrm{Na}^{+}\right)$was obtained according to Procedure B from $\mathbf{1 d}(36 \mathrm{mg}, 0.20 \mathrm{mmol})$ and $\mathbf{2 n}-\mathrm{H}(13 \mathrm{mg}, 0.20 \mathrm{mmol})$.

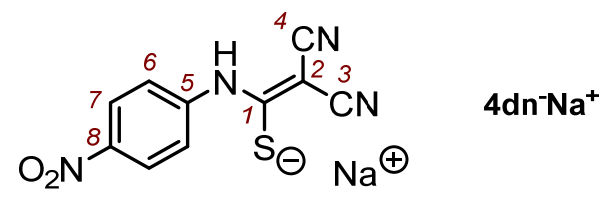

${ }^{1}$ H NMR (400 MHz, d6-DMSO): $\delta 9.65$ (s, 1 H, NH), 8.09 (d, $J=9.3 \mathrm{~Hz}, 2 \mathrm{H}, 7$-H), 7.82 (d, $J$ $=9.3 \mathrm{~Hz}, 2 \mathrm{H}, 6-\mathrm{H}) .{ }^{13} \mathrm{C}$ NMR (101 MHz, $d_{6}$-DMSO): $\delta 188.7$ (C-1), 148.3 (C-5), 141.1 (C-8), 123.8 (C-7), 121.2 (C-6), 121.1 (C-3 or C-4), 119.1 (C-3 or C-4), 55.9 (C-2). HRMS (ESI'): $m / z$ calcd for $\left[\mathrm{C}_{10} \mathrm{H}_{5} \mathrm{~N}_{4} \mathrm{O}_{2} \mathrm{~S}^{-}\right]\left(\mathbf{4 d n}^{-}\right): 245.0139$, found: 245.0137.

Sodium (Z)-2-(ethoxycarbonyl)-1-((4-nitrophenyl)amino)-3-oxobut-1-ene-1-thiolate (4do$\mathbf{N a}^{+}$) was obtained according to Procedure B from 1d (36 mg, $\left.0.20 \mathrm{mmol}\right)$ and $\mathbf{2 o}-\mathrm{H}(26 \mathrm{mg}$, $0.20 \mathrm{mmol})$.

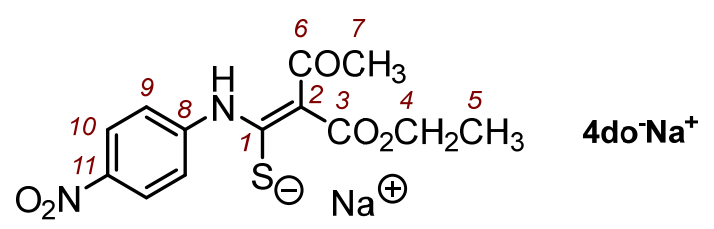

${ }^{1}$ H NMR (400 MHz, $d_{6}$-DMSO): $\delta 15.00$ (s, 1 H, NH), 8.22 (d, $J=9.4$ Hz, 2 H, 10-H), 8.11 (d, $J=9.4 \mathrm{~Hz}, 2 \mathrm{H}, 9-\mathrm{H}), 4.05$ (q, $J=7.1 \mathrm{~Hz}, 2 \mathrm{H}, 4-\mathrm{H}), 1.81$ (s, $3 \mathrm{H}, 7-\mathrm{H}), 1.21$ (t, $J=7.1 \mathrm{~Hz}, 3$ H, 5-H). ${ }^{13}$ C NMR (101 MHz, d6-DMSO): $\delta 183.8$ (C-1), 180.8 (C-6), 171.4 (C-3), 148.8 (C11), 140.6 (C-8), 124.4 (C-9), 119.9 (C-10), 114.6 (C-2), 59.4 (C-4), 26.7 (C-7), 14.0 (C-5). HRMS (ESI $\left.{ }^{-}\right): m / z$ calcd for $\left[\mathrm{C}_{13} \mathrm{H}_{13} \mathrm{~N}_{2} \mathrm{O}_{5} \mathrm{~S}^{-}\right]\left(\mathbf{4 d o}^{-}\right)$: 309.0551, found: 309.0554 .

Sodium 2-acetyl-1-((4-nitrophenyl)amino)-3-oxobut-1-ene-1-thiolate $\quad\left(4 \mathrm{dp}^{-} \mathrm{Na}^{+}\right)$was obtained according to Procedure B from 1d (36 mg, $0.20 \mathrm{mmol})$ and 2p-H (20 mg, $0.20 \mathrm{mmol})$.<smiles>CC(=O)C(C(=O)Nc1ccc([N+](=O)[O-])cc1)=C([O-])[N+]#[N+]</smiles>

${ }^{1}$ H NMR (400 MHz, d6-DMSO): $\delta 15.14$ (s, $\left.1 \mathrm{H}, \mathrm{NH}\right), 8.20$ (d, $\left.J=9.3 \mathrm{~Hz}, 2 \mathrm{H}, 7-\mathrm{H}\right), 8.11$ (d, $J=9.3 \mathrm{~Hz}, 2 \mathrm{H}, 6-\mathrm{H}), 2.11$ (s, $6 \mathrm{H}, 4-\mathrm{H}) .{ }^{13} \mathbf{C}$ NMR (101 MHz, d6-DMSO): $\delta 203.5$ (C-3), $\delta$ 183.8 (C-1), 148.7 (C-8), 140.7 (C-5), 124.3 (C-6), 121.9 (C-2), 120.1 (C7), 30.0 (C-4). HRMS $\left(\mathrm{ESI}^{-}\right): \mathrm{m} / z$ calcd for $\left[\mathrm{C}_{12} \mathrm{H}_{11} \mathrm{~N}_{2} \mathrm{O}_{4} \mathrm{~S}\right]^{-}\left(\mathbf{4} \mathbf{d p}^{-}\right): 279.0445$, found: 279.0444 . 


\subsection{Products of Reactions of Nucleophiles with $\mathrm{PhN}=\mathrm{C}=\mathrm{NPh}(1 \mathrm{e})$}

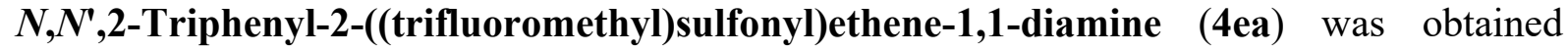
according to Procedure $\mathrm{C}$ from $1 \mathrm{e}(39 \mathrm{mg}, 0.20 \mathrm{mmol})$ and $\mathbf{2 a}-\mathrm{H}(45 \mathrm{mg}, 0.20 \mathrm{mmol})$ : white solid (79 mg, 94\%), mp 129-134 ${ }^{\circ} \mathrm{C}$.

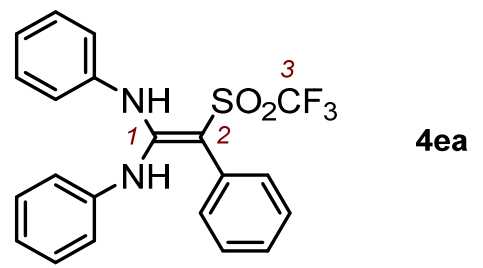

${ }^{1}$ H NMR (400 MHz, $d_{6}$-DMSO): $\delta 9.13$ (s, 2 H, NH), 7.17-7.15 (m, 4 H), 7.12-7.04 (m, 5 H), 7.01-6.98 (m, $4 \mathrm{H}), 6.87$ (t, $J=7.3 \mathrm{~Hz}, 2 \mathrm{H}) .{ }^{13} \mathbf{C}$ NMR (101 MHz, $d_{6}$-DMSO): $\delta 155.5$ (C-1), $139.1(\mathrm{C}), 133.2(\mathrm{C}), 131.8(\mathrm{CH}), 128.6(\mathrm{CH}), 127.8(\mathrm{CH}), 126.0(\mathrm{CH}), 123.6(\mathrm{CH}), 120.78(\mathrm{q}$, $\left.J_{\mathrm{C}, \mathrm{F}}=330 \mathrm{~Hz}, \mathrm{C}-3\right), 120.75(\mathrm{CH}), 81.5\left(\mathrm{q}, J_{\mathrm{C}, \mathrm{F}}=2.0 \mathrm{~Hz}, \mathrm{C}-2\right)$. HRMS (EI): $m / z$ calcd for $\left[\mathrm{C}_{21} \mathrm{H}_{17} \mathrm{~F}_{3} \mathrm{~N}_{2} \mathrm{O}_{2} \mathrm{~S}^{++}\right]\left(\mathrm{M}^{++}\right): 418.0957$, found: 418.0955. IR (ATR): v 3390, 3346, 1598, 1576, $1495,1440,1378,1313,1195,1183,1169,1115,951,751,689,660 \mathrm{~cm}^{-1}$.

Ethyl 2-(4-cyanophenyl)-3,3-bis(phenylamino)acrylate (4ef) was obtained in analogy to Procedure $\mathrm{C}$ but independently synthesized $\mathbf{2 f}-\mathrm{K}$ was used rather than generation by $\mathrm{KO} t \mathrm{Bu}$ with $\mathbf{2 e - H}$. The product was obtained from 1 e $(39 \mathrm{mg}, 0.20 \mathrm{mmol})$ and $\mathbf{2 f}-\mathrm{K}(45 \mathrm{mg}, 0.20 \mathrm{mmol})$ : pale yellow solid (57 mg, 74\%), mp 150-155 ${ }^{\circ} \mathrm{C}$.

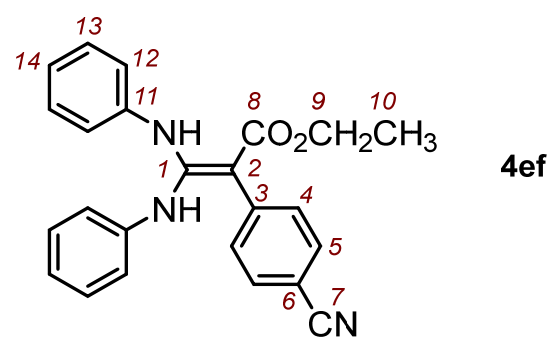

${ }^{1}$ H NMR (400 MHz, d6-DMSO): $\delta 9.08$ (s, 2 H, NH), 7.52 (d, $J=8.4$ Hz, 2 H, 5-H), 7.31 (d, $J$ $=8.4 \mathrm{~Hz}, 2 \mathrm{H}, 4-\mathrm{H}), 7.09$ (t, $J=7.6 \mathrm{~Hz}, 4 \mathrm{H}, 13-\mathrm{H}), 6.97$ (d, $J=7.5 \mathrm{~Hz}, 4 \mathrm{H}, 12-\mathrm{H}), 6.79$ (t, $J$ $=7.3 \mathrm{~Hz}, 2 \mathrm{H}, 14-\mathrm{H}), 3.92(\mathrm{q}, J=7.1 \mathrm{~Hz}, 2 \mathrm{H}, 9-\mathrm{H}), 1.03$ (t, $J=7.1 \mathrm{~Hz}, 3 \mathrm{H}, 10-\mathrm{H}) .{ }^{13} \mathbf{C}$ NMR (101 MHz, d6-DMSO): $\delta 168.3$ (C-8), 152.0 (C-1), 144.1 (C-3), 141.1 (C-11), 131.4 (C-4), 131.1 (C-5), 128.7 (C-13), 121.8 (C-14), 119.6 (C-7), 118.8 (C-12), 106.1 (C-6), 90.4 (C-2), 58.8 (C-9), 14.3 (C-10). HRMS (EI): $m / z$ calcd for $\left[\mathrm{C}_{24} \mathrm{H}_{21} \mathrm{~N}_{3} \mathrm{O}_{2}{ }^{++}\right]\left(\mathrm{M}^{+}\right): 383.1628$, found: 383.1627. IR (ATR): v 3336, 2229, 1628, 1612, 1596, 1579, 1497, 1460, 1432, 1371, 1226, $1202,1058,749,698 \mathrm{~cm}^{-1}$. 
4-(1-Cyano-2,2-bis(phenylamino)vinyl)benzonitrile (4eh) was obtained according to Procedure C from 1e (39 mg, $0.20 \mathrm{mmol})$ and $\mathbf{2 h}-\mathrm{H}$ (28 mg, $0.20 \mathrm{mmol})$ : yellow solid (57 mg, $85 \%), \operatorname{mp} 235-240{ }^{\circ} \mathrm{C}$.

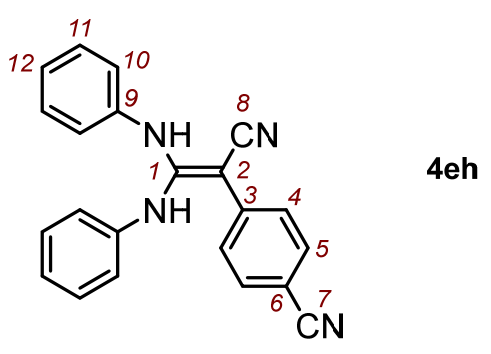

${ }^{1} \mathbf{H}$ NMR (400 MHz, d6-DMSO): $\delta 9.47$ (s, $\left.2 \mathrm{H}, \mathrm{NH}\right), 7.57$ (d, $J=8.5 \mathrm{~Hz}, 2 \mathrm{H}, 5$-H), 7.33 (d, $J$ $=8.5 \mathrm{~Hz}, 2 \mathrm{H}, 4-\mathrm{H}$ ), 7.17 (br s, $4 \mathrm{H}, 11-\mathrm{H}), 7.03$ (br s, $4 \mathrm{H}, 10-\mathrm{H}), 6.88$ (br s, $2 \mathrm{H}, 12-\mathrm{H}) .{ }^{13} \mathbf{C}$ NMR (101 MHz, $d_{6}$-DMSO): $\delta 151.5$ (C-1), 141.5 (C-3), 140.4 (C-9), 132.1 (C-5), 129.0 (C11), 125.5 (C-4), 122.4 (C-12), 121.5 (C-8), 119.4 (C-7), 118.8 (C-10), 104.9 (C-6), 70.4 (C2). HRMS (EI): $m / z$ calcd for $\left[\mathrm{C}_{22} \mathrm{H}_{16} \mathrm{~N}_{4}{ }^{+}\right]\left(\mathrm{M}^{++}\right): 336.1369$, found: 336.1378 . IR (ATR): $v$ $3308,3050,2224,2191,1611,1594,1569,1528,1496,1480,1362,1249,1178,844,762,739$, $689 \mathrm{~cm}^{-1}$.

Diethyl 2-(bis(phenylamino)methylene)malonate (4em) was obtained according to Procedure C from 1e (39 mg, $0.20 \mathrm{mmol})$ and $\mathbf{2 m}-\mathrm{H}(32 \mathrm{mg}, 0.20 \mathrm{mmol})$ : white solid $(64 \mathrm{mg}$, 90\%), mp 171-173 ${ }^{\circ} \mathrm{C}$ (lit.: mp $169.9{ }^{\circ} \mathrm{C}^{\mathrm{S} 10 \mathrm{a}}$; mp $167{ }^{\circ} \mathrm{C}^{\mathrm{S} 10 \mathrm{~b}}$ ). Spectroscopic data agree with those reported in the literature. ${ }^{\text {s10a }}$

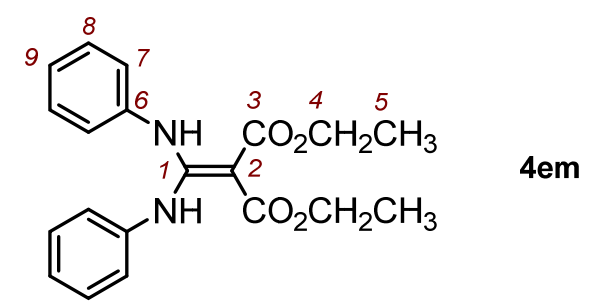

${ }^{1}$ H NMR (400 MHz, $\left.\mathrm{CDCl}_{3}\right): \delta 11.24$ (s, $\left.2 \mathrm{H}, \mathrm{NH}\right), 6.99$ (d, $\left.J=7.3 \mathrm{~Hz}, 4 \mathrm{H}, 8-\mathrm{H}\right), 6.90$ (d, $J=$ $7.4 \mathrm{~Hz}, 4 \mathrm{H}, 7-\mathrm{H}), 6.82$ (t, $J=7.3 \mathrm{~Hz}, 2 \mathrm{H}, 9-\mathrm{H}), 4.24$ (q, $J=7.1 \mathrm{~Hz}, 4 \mathrm{H}, 4-\mathrm{H}), 1.34$ (t, $J=7.1$ $\mathrm{Hz}, 6 \mathrm{H}, 5-\mathrm{H}) .{ }^{13} \mathbf{C}$ NMR (101 MHz, $\left.\mathrm{CDCl}_{3}\right): \delta 171.5$ (C-3), 159.0 (C-1), 138.3 (C-6), 128.6 (C-8), 124.3 (C-9), 122.4 (C-7), 80.1 (C-2), 60.3 (C-4), 14.5 (C-5). HRMS (EI): $m / z$ calcd for $\left[\mathrm{C}_{20} \mathrm{H}_{22} \mathrm{~N}_{2} \mathrm{O}_{4}{ }^{++}\right]\left(\mathrm{M}^{+}\right): 354.1574$, found: 354.1578. IR (ATR): v 2978, 1626, 1585, 1512, 1438 , $1398,1373,1342,1223,1176,1086,1066,1024,748,704 \mathrm{~cm}^{-1}$.

Ethyl 2-(bis(phenylamino)methylene)-3-oxobutanoate (4eo) was obtained according to Procedure C from 1e (39 mg, $0.20 \mathrm{mmol})$ and $\mathbf{2 0}-\mathrm{H}(26 \mathrm{mg}, 0.20 \mathrm{mmol})$ : white solid (52 $\mathrm{mg}$, 80\%), mp $107-112{ }^{\circ} \mathrm{C}$ (lit.: $\mathrm{mp} 107-108^{\circ} \mathrm{C}^{\mathrm{S} 11}$; mp $109^{\circ} \mathrm{C}^{\mathrm{S} 10 \mathrm{~b}}$ ). 


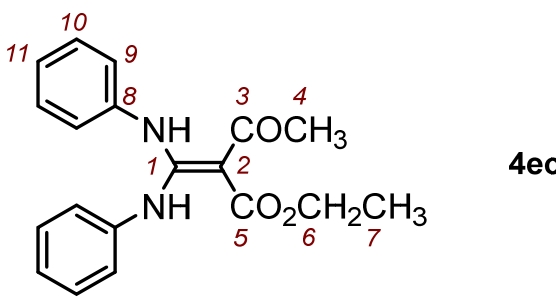

${ }^{1}$ H NMR (400 MHz, d6-DMSO): $\delta 11.95$ (s, 2 H, NH), 7.07 (t, J= 7.7 Hz, 4 H, 10-H), 6.99 (d, $J=7.9 \mathrm{~Hz}, 4 \mathrm{H}, 9-\mathrm{H}), 6.88$ (t, $J=7.3 \mathrm{~Hz}, 2 \mathrm{H}, 11-\mathrm{H}), 4.08$ (q, $J=7.1 \mathrm{~Hz}, 2 \mathrm{H}, 6-\mathrm{H}), 2.35$ (s, 3 H, 4-H), 1.21 (t, $J=7.1 \mathrm{~Hz}, 3 \mathrm{H}, 7-\mathrm{H}) .{ }^{13} \mathrm{C}$ NMR (101 MHz, $d_{6}$-DMSO): $\delta 196.2$ (C-3), 170.3 (C-5), 158.0 (C-1), 137.7 (C-8), 128.6 (C-10), 124.4 (C-11), 122.0 (C-9), 91.4 (C-2), 59.7 (C6), 31.5 (C-4), 14.2 (C-7). HRMS (EI): $m / z$ calcd for $\left[\mathrm{C}_{19} \mathrm{H}_{20} \mathrm{~N}_{2} \mathrm{O}_{3}{ }^{+{ }^{+}}\right]\left(\mathrm{M}^{+}\right)$: 324.1468 , found: 324.1471. IR (ATR): v 2976, 1623, 1572, 1516, 1333, 1247, 1225, 1170, 1086, 1076, 1018, $971,786,758,748,690 \mathrm{~cm}^{-1}$.

\subsection{Products of Reactions of Nucleophiles with $\mathrm{CS}_{2}(1 \mathrm{~g})$}

Potassium 2-(4-cyanophenyl)-3-ethoxy-3-oxopropanedithioate $\left(\operatorname{tgf}^{-} \mathrm{K}^{+}\right)$was obtained in analogy to Procedure B but with independently synthesized $\mathbf{2 f - K}$ (instead of the combination $\mathrm{NaOH}+\mathbf{2 f}-\mathrm{H})$. The product was obtained from $\mathbf{1 g}(15 \mathrm{mg}, 0.20 \mathrm{mmol})$ and $\mathbf{2 f}-\mathrm{K}(45 \mathrm{mg}, 0.20$ mmol).

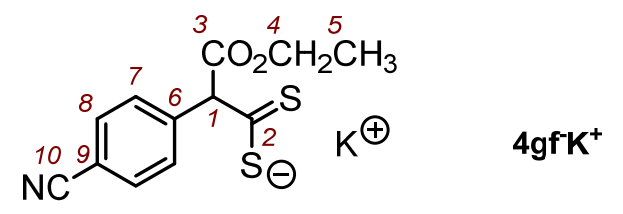

${ }^{1}$ H NMR (400 MHz, d6-DMSO): $\delta 7.77$ (d, $J=8.5$ Hz, 2 H, 7-H), 7.69 (d, $J=8.6$ Hz, 2 H, 8H), 5.42 (s, 1 H, 1-H), 4.06-3.99 (m, 2 H, 4-H), 1.16 (t, J = 7.1 Hz, 3 H, 5-H). ${ }^{13}$ C NMR (101 MHz, d6-DMSO): $\delta 192.7$ (C-2), 168.6 (C-3), 144.0 (C-6), 131.0 (C-8), 130.1 (C-7), 119.2 (C10), 109.1 (C-9), 79.0 (C-1), 59.9 (C-4), 14.0 (C-5).

Potassium 2-(4-nitrophenyl)-3-ethoxy-3-oxopropanedithioate $\left(4 \mathrm{gg}^{-} \mathrm{K}^{+}\right)$was obtained in analogy to Procedure $\mathrm{C}$ but independently synthesized $\mathbf{2 g}-\mathrm{K}$ was used rather than generation in situ by $\mathrm{NaOH}$ with $\mathbf{2 g}-\mathrm{H}$. The product was obtained from $\mathbf{1 g}$ (15 mg, $0.20 \mathrm{mmol}$ ) and $\mathbf{2 g}-\mathrm{K}$ (49 $\mathrm{mg}, 0.20 \mathrm{mmol})$.<smiles>CCOC(=O)C(=S)C(=S)OCC</smiles>

${ }^{1} \mathbf{H}$ NMR (400 MHz, $d_{6}$-DMSO): $\delta 8.12(\mathrm{~d}, J=8.4 \mathrm{~Hz}, 2 \mathrm{H}), 7.85(\mathrm{~d}, J=8.4 \mathrm{~Hz}, 2 \mathrm{H}), 5.50$ (s, $1 \mathrm{H}), 4.04(\mathrm{~m}, 2 \mathrm{H}), 1.17$ (t, $J=7.0 \mathrm{~Hz}, 3 \mathrm{H})$. 
Sodium (E)-2-cyano-1-mercapto-2-(4-nitrophenyl)ethene-1-thiolate (4gi-Na ${ }^{+}$was obtained according to Procedure B from $1 \mathrm{~g}$ (15 mg, $0.20 \mathrm{mmol})$ and $\mathbf{2 i}-\mathrm{H}$ (32 mg, $0.20 \mathrm{mmol})$.<smiles>N#CC(C([O-])=S)c1ccc([N+](=O)[O-])cc1</smiles>

$4 \mathrm{gi}^{-} \mathrm{Na}^{+}$

${ }^{1}$ H NMR (400 MHz, d6-DMSO): $\delta 8.52(\mathrm{~d}, J=9.3 \mathrm{~Hz}, 2 \mathrm{H}), 7.98(\mathrm{~d}, J=9.3 \mathrm{~Hz}, 2 \mathrm{H}), 5.44$ (br s, $1 \mathrm{H})$.

((1H-Inden-1-ylidene)methylene)bis(methylsulfane) (6gj) and methyl 1(bis(methylthio)methylene)-1H-indene-3-carbodithioate (7gj). To a solution of $\mathrm{KO} t \mathrm{Bu}$ (56 $\mathrm{mg}, 0.50 \mathrm{mmol})$ in anhydrous DMSO $(1 \mathrm{~mL})$ at room temperature were added a solution of $\mathbf{2} \mathbf{j}$ $\mathrm{H}(58 \mathrm{mg}, 0.50 \mathrm{mmol})$ in anhydrous DMSO $(0.5 \mathrm{~mL})$ and then a solution of $1 \mathrm{~g}(76 \mathrm{mg}, 1.0$ $\mathrm{mmol})$ in anhydrous DMSO $(0.5 \mathrm{~mL})$. After $20 \mathrm{~min}$, a solution of iodomethane $(284 \mathrm{mg}, 2.00$ mmol) in $0.5 \mathrm{~mL}$ anhydrous DMSO was added and the resulting solution stirred at room temperature for $30 \mathrm{~min}$. Afterwards, $20 \mathrm{~mL}$ of saturated $\mathrm{NH}_{4} \mathrm{Cl}$ solution was added and the mixture was extracted with EtOAc $(3 \times 30 \mathrm{~mL})$. The combined organic phase was washed with water $(2 \times 30 \mathrm{~mL})$ and brine $(1 \times 30 \mathrm{~mL})$, dried with anhydrous $\mathrm{MgSO}_{4}$, and filtered. The solvent was evaporated under reduced pressure, and the residue was purified by column chromatography to furnish $\mathbf{7 g j}$ as a red viscous liquid (84 $\mathrm{mg}, 54 \%)$ and $\mathbf{6 g j}{ }^{\mathrm{S12}}$ as a yellow viscous liquid (33 mg, 30\%).
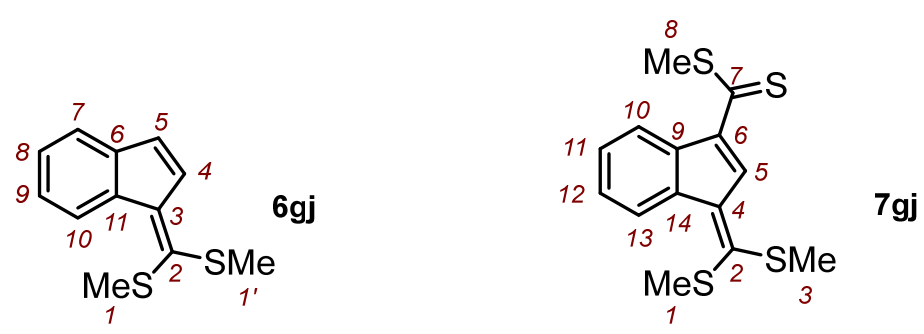

6gj: ${ }^{1} \mathbf{H}$ NMR (400 MHz, $\left.\mathrm{CDCl}_{3}\right): \delta 8.59-8.51$ (m, $\left.1 \mathrm{H}, 7-\mathrm{H}\right), 7.38-7.31$ (m, $\left.1 \mathrm{H}, 10-\mathrm{H}\right), 7.30$ 7.24 (m, 2 H, 8-H and 9-H), 7.17 (d, $J=4.5$ Hz, 1 H, 5-H), 6.82 (d, J=5.4 Hz, 1 H, 4-H), 2.63 (s, 3 H, 1-H or $\left.1^{6}-\mathrm{H}\right), 2.55$ (s, $3 \mathrm{H}, 1-\mathrm{H}$ or $\left.1^{6}-\mathrm{H}\right) .{ }^{13} \mathbf{C ~ N M R}\left(101 \mathrm{MHz}, \mathrm{CDCl}_{3}\right)$ : $\delta 144.8$ (C-6), 144.4 (C-2), 143.3 (C-3), 134.7 (C-11), 129.9 (C-4), 129.1 (C-5), 127.2 (C-9), 125.4 (C-8), 125.2 (7), $121.2(\mathrm{C}-10), 18.7\left(\mathrm{C}-1\right.$ or $\left.^{\mathrm{C}}-1^{\circ}\right), 18.0\left(\mathrm{C}-1\right.$ or $\left.\mathrm{C}-1^{\circ}\right)$. HRMS (EI): $m / z$ calcd for $\left[\mathrm{C}_{12} \mathrm{H}_{12} \mathrm{~S}^{++}\right]\left(\mathrm{M}^{++}\right): 220.0375$, found: 220.0379. IR (ATR): $v$ 3057, 2918, 1650, 1530, 1442, $1361,1309,1187,1106,1074,1020,961,898,790,749,738,721 \mathrm{~cm}^{-1}$.

7gj: ${ }^{1} \mathbf{H}$ NMR (400 MHz, $\left.\mathrm{CDCl}_{3}\right): \delta 8.44(\mathrm{dd}, J=5.7 \mathrm{~Hz}, 3.2 \mathrm{~Hz}, 1 \mathrm{H}, 11-\mathrm{H}$ or 12-H), 8.23 (dd, $J=5.1 \mathrm{~Hz}, 3.3 \mathrm{~Hz}, 1 \mathrm{H}, 11-\mathrm{H}$ or 12-H), 7.83 (s, $1 \mathrm{H}, 5-\mathrm{H}), 7.29-7.27$ (m, $2 \mathrm{H}, 10-\mathrm{H}$ and 13-H), 
2.75 (s, 3 H, 8-H), 2.65 (s, 3 H, 1-H or 3-H), 2.57 (s, 3 H, 1-H or 3-H). ${ }^{13}$ C NMR (101 MHz, $\left.\mathrm{CDCl}_{3}\right): \delta 221.2(\mathrm{C}-7), 154.0(\mathrm{C}-2), 144.7,141.3,140.0,135.1,129.4(\mathrm{C}-5), 127.1$ (C-10 or C13), 126.2 (C-10 or C-13), 124.7 (C-11 or C-12), 122.9 (C-11 or C-12), 19.6 (C-1 or C-3), 19.1 (C-2), 18.8 (C-1 or C-3). HRMS (EI): $m / z$ calcd for $\left[\mathrm{C}_{14} \mathrm{H}_{14} \mathrm{~S}_{4}{ }^{+}\right]\left(\mathrm{M}^{+}\right)$: 309.9973, found: 309.9974. IR (ATR): v 2916, 1493, 1454, 1432, 1306, 1238, 1192, 1132, 1108, 1077, 1020, $908,795,749 \mathrm{~cm}^{-1}$.

\subsection{Products of Reactions of Nucleophiles with $\mathrm{CO}_{2}(1 \mathrm{j})$}

$\mathbf{1 H}$-Indene-3-carboxylic acid (4hj). To a solution of $\mathrm{KO} t \mathrm{Bu}(190 \mathrm{mg}, 1.70 \mathrm{mmol})$ in anhydrous DMSO (3 mL) at room temperature was added a solution of $\mathbf{2} \mathbf{j}-\mathrm{H}(197 \mathrm{mg}, 1.70$ mmol) in anhydrous DMSO $(2 \mathrm{~mL})$. Afterwards, excess solid $\mathrm{CO}_{2}$ was added separately $(3 \times)$. After $30 \mathrm{~min}, 30 \mathrm{~mL}$ of $2 \mathrm{M} \mathrm{HCl}$ solution was added and the mixture was extracted with EtOAc $(3 \times 30 \mathrm{~mL})$. The combined organic phase was washed with water $(2 \times 30 \mathrm{~mL})$, brine $(1 \times 30$ $\mathrm{mL}$ ), dried with anhydrous $\mathrm{MgSO}_{4}$, and filtered. The solvent was evaporated under reduced pressure, and the residue was purified by column chromatography to give $\mathbf{4 h \mathbf { j }}$ as a white solid (218 mg, 80\%), mp $163-168{ }^{\circ} \mathrm{C}$ (lit.: $\mathrm{mp} 161-162{ }^{\circ} \mathrm{C}^{\mathrm{S} 13}$ ). Spectroscopic data agree with those reported in the literature. ${ }^{\mathrm{S} 13}$

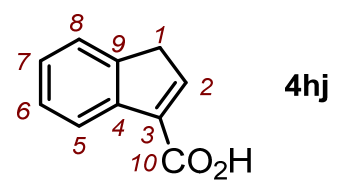

${ }^{1} \mathbf{H}$ NMR (400 MHz, $\mathrm{CDCl}_{3}$ ): $\delta 11.44$ (br s, $\left.1 \mathrm{H}, \mathrm{COOH}\right), 8.11$ (d, J=7.6 Hz, $\left.1 \mathrm{H}, 8-\mathrm{H}\right), 7.66$ (t, $J=2.0 \mathrm{~Hz}, 1 \mathrm{H}, 2-\mathrm{H}), 7.51$ (d, $J=7.4 \mathrm{~Hz}, 1 \mathrm{H}, 5-\mathrm{H}), 7.40$ (t, $J=7.6 \mathrm{~Hz}, 1 \mathrm{H}, 7-\mathrm{H}), 7.30$ (t, $J=7.4 \mathrm{~Hz}, 1 \mathrm{H}, 6-\mathrm{H}), 3.59$ (d, $J=2.0 \mathrm{~Hz}, 2 \mathrm{H}, 1-\mathrm{H}) .{ }^{13} \mathbf{C}$ NMR $\left(101 \mathrm{MHz}, \mathrm{CDCl}_{3}\right): \delta 169.8$ (C-10), 147.3 (C-2), 143.5 (C-3), 140.5 (C-4), 135.9 (C-9), 126.9 (C-7), 125.9 (C-6), 124.0 (C5), 122.7 (C-8), 38.8 (C-1). HRMS (EI): $\mathrm{m} / z$ calcd for $\left[\mathrm{C}_{10} \mathrm{H}_{8} \mathrm{O}_{2}{ }^{-+}\right]\left(\mathrm{M}^{+}\right): 160.0519$, found: 160.0522. IR (ATR): $v$ 3041, 2889, 2779, 2602, 1679, 1567, 1464, 1427, 1372, 1258, 1220, $1017,964,905,767,733,707 \mathrm{~cm}^{-1}$. 
Chart S1. Carbanions Not Reacting with $\mathrm{CO}_{2}$ in DMSO (with $N$ and $s \mathrm{~N}$ from $\operatorname{ref}^{\mathrm{S} 14}$ and $\mathrm{p} K \mathrm{aH}$ in DMSO from $\mathrm{ref}^{\mathrm{S} 15}$ )<smiles>O=C(O[Sb]c1ccccc1)OC(F)(F)F</smiles>

$2 a$

$\mathrm{p} K_{\mathrm{aH}} 14.6$

$N=18.67 s_{N}=0.68$<smiles>CCOC(=O)Cc1ccncc1</smiles>

$N=23.27 s_{N}=0.70$

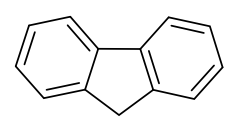

$\mathrm{p} K_{\mathrm{aH}} 22.6$<smiles>CCOC(=O)Cc1ccccc1</smiles>

$2 e$ $N=27.54 s_{N}=0.57$<smiles>N#Cc1ccc(C[N+](=O)[O-])cc1</smiles>

$\mathrm{p} K_{\mathrm{aH}} 9.31$ $N=16.96 s_{N}=0.73$<smiles>CCOC(=O)Cc1ccc(C#N)cc1</smiles>

$2 f$<smiles>N#CCc1ccc([N+](=O)[O-])cc1</smiles>
$\mathrm{p} K_{\mathrm{aH}} 12.3$

$N=23.64 s_{N}=0.65 \quad N=19.67 s_{N}=0.68$<smiles>N#Cc1ccc(CS(=O)(=O)c2ccccc2)cc1</smiles><smiles>O=C(Cc1ccccc1)c1ccccc1</smiles>

$N=22.60 s_{N}=0.57$ $N=23.15 s_{N}=0.60$

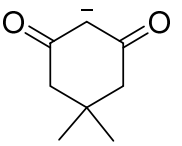

$\mathrm{p} K_{\mathrm{aH}} 11.2$ $N=16.27 s_{N}=0.77$<smiles>CCOC(=O)CP(=O)(c1ccccc1)c1ccccc1</smiles>

$\mathrm{p} K_{\mathrm{aH}} 18.9$ $N=19.20 s_{N}=0.69$<smiles>N#CCP(=O)(c1ccccc1)c1ccccc1</smiles>

$\mathrm{p} K_{\mathrm{aH}} 16.9$ $N=18.69 s_{N}=0.72$<smiles>C1=C(c2ccccc2)Cc2ccccc21</smiles>

$\mathrm{p} K_{\mathrm{aH}} 19.37$ 


\section{Kinetics of the Reactions of the Heteroallenes 1 with Carbanions 2 and Enamines 3}

The rates of all investigated reactions were determined spectrophotometrically (UV-Vis) by following the disappearance of the UV/Vis absorptions of the nucleophiles $\mathbf{2}, \mathbf{3}$, or the increase of the UV/Vis absorptions of the products 4 . The temperature of the solutions during all kinetics studies was kept constant $\left(20.0 \pm 0.1^{\circ} \mathrm{C}\right)$ by using a circulating bath thermostat. The kinetics investigations were performed with a high excess of the one component over the other to achieve pseudo-first-order kinetics. The kinetics were followed by using a conventional UV/Vis diode array spectrophotometer (slow kinetics, $\tau_{1 / 2}>10 \mathrm{~s}$ ) or a commercial stopped-flow spectrophotometer system (fast kinetics, $\tau_{1 / 2}<10 \mathrm{~s}$ ). Rate constants $k_{\mathrm{obs}}\left(\mathrm{s}^{-1}\right)$ were obtained by fitting the single exponential decay function $A_{\mathrm{t}}=A_{0} \exp \left(-k_{\mathrm{obs}} t\right)+C$ (exponential decrease) or the single exponential increase function $A_{\mathrm{t}}=A_{\infty}\left[1-\exp \left(-k_{\mathrm{obs}} t\right)\right]+C$ (exponential increase) to the observed time-dependent absorbance (in the case of the stopped-flow kinetics, at least 5 kinetic runs for each electrophile or nucleophile concentration were averaged). Second-order rate constants $k_{2}\left(\mathrm{M}^{-1} \mathrm{~s}^{-1}\right)$ were derived from the slopes of the linear correlations of $k_{\mathrm{obs}}$ with electrophile concentrations. 
Table S1. Kinetics of the reactions of $\mathbf{1 a}$ with $\mathbf{2 b}$ in $\mathrm{CH}_{3} \mathrm{CN}$ at $20^{\circ} \mathrm{C}$ (deprotonated with $1.00-1.05$ equiv. $\mathrm{KO} t \mathrm{Bu}$, conventional $\mathrm{UV}-\mathrm{Vis}$ spectrometer, $\lambda=320 \mathrm{~nm}$ ).

\begin{tabular}{ccc}
{$[\mathbf{2 b}] / \mathbf{M}$} & {$[\mathbf{1 a}] / \mathbf{M}$} & $\boldsymbol{k}_{\mathbf{o b s}} / \mathbf{s}^{\mathbf{- 1}}$ \\
$5.00 \times 10^{-5}$ & $4.59 \times 10^{-4}$ & $9.23 \times 10^{-3}$ \\
$5.00 \times 10^{-5}$ & $6.93 \times 10^{-4}$ & $1.44 \times 10^{-2}$ \\
$5.00 \times 10^{-5}$ & $9.04 \times 10^{-4}$ & $2.08 \times 10^{-2}$ \\
$5.00 \times 10^{-5}$ & $1.13 \times 10^{-3}$ & $2.54 \times 10^{-2}$ \\
$5.00 \times 10^{-5}$ & $1.34 \times 10^{-3}$ & $3.11 \times 10^{-2}$ \\
\hline \multicolumn{3}{c}{$\left.\boldsymbol{k}_{\mathbf{2}}=\mathbf{( 2 . 4 9} \pm \mathbf{0 . 0 8}\right) \times \mathbf{1 0}^{\mathbf{1}} \mathbf{M}^{-\mathbf{1}} \mathbf{s}^{\mathbf{1}}$}
\end{tabular}

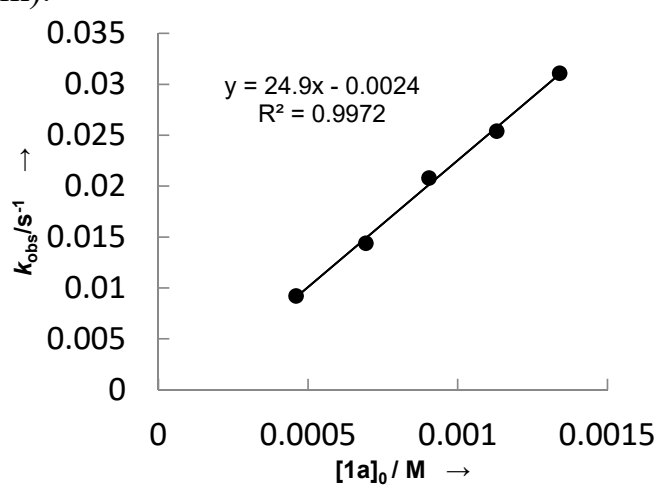

Table S2. Kinetics of the reactions of $\mathbf{1 a}$ with $\mathbf{2} \mathbf{c}$ in $\mathrm{CH}_{3} \mathrm{CN}$ at $20^{\circ} \mathrm{C}$ (deprotonated with $1.00-1.05$ equiv. $\mathrm{KO} t \mathrm{Bu}$, conventional $\mathrm{UV}-\mathrm{Vis}$ spectrometer, $\lambda=344 \mathrm{~nm}$ ).

\begin{tabular}{|c|c|c|}
\hline$[2 c] / M$ & {$[\mathbf{1 a}] / \mathbf{M}$} & $k_{\text {obs }} / \mathbf{s}^{-1}$ \\
\hline $5.00 \times 10^{-5}$ & $5.43 \times 10^{-4}$ & $1.14 \times 10^{-3}$ \\
\hline $5.00 \times 10^{-5}$ & $8.20 \times 10^{-4}$ & $1.86 \times 10^{-3}$ \\
\hline $5.00 \times 10^{-5}$ & $1.07 \times 10^{-3}$ & $2.53 \times 10^{-3}$ \\
\hline $5.00 \times 10^{-5}$ & $1.34 \times 10^{-3}$ & $3.25 \times 10^{-3}$ \\
\hline
\end{tabular}

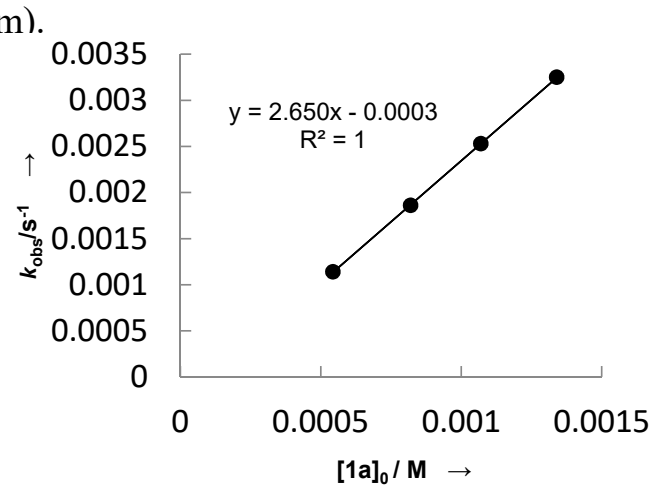

Table S3. Kinetics of the reactions of $\mathbf{1 a}$ with $\mathbf{2 d}$ in $\mathrm{CH}_{3} \mathrm{CN}$ at $20^{\circ} \mathrm{C}$ (deprotonated with 1.00-1.05 equiv. $\mathrm{KO} t \mathrm{Bu}$, conventional $\mathrm{UV}$-Vis spectrometer, $\lambda=464 \mathrm{~nm}$ ).

\begin{tabular}{ccc}
{$[\mathbf{2 d}] / \mathbf{M}$} & {$[\mathbf{1 a}] / \mathbf{M}$} & $\boldsymbol{k}_{\mathbf{o b s}} / \mathbf{s}^{-\mathbf{1}}$ \\
$5.00 \times 10^{-5}$ & $6.00 \times 10^{-4}$ & $1.33 \times 10^{-4}$ \\
$5.00 \times 10^{-5}$ & $1.16 \times 10^{-3}$ & $3.39 \times 10^{-4}$ \\
$5.00 \times 10^{-5}$ & $1.78 \times 10^{-3}$ & $5.06 \times 10^{-4}$ \\
$5.00 \times 10^{-5}$ & $2.31 \times 10^{-3}$ & $6.22 \times 10^{-4}$ \\
$5.00 \times 10^{-5}$ & $2.79 \times 10^{-3}$ & $9.05 \times 10^{-4}$ \\
\hline \multicolumn{3}{c}{$\left.\boldsymbol{k}_{\mathbf{2}}=\mathbf{( 3 . 2 9} \pm \mathbf{0 . 0 3}\right) \times \mathbf{1 0}^{-\mathbf{1}} \mathbf{M}^{-\mathbf{1}} \mathbf{s}^{-1}$}
\end{tabular}

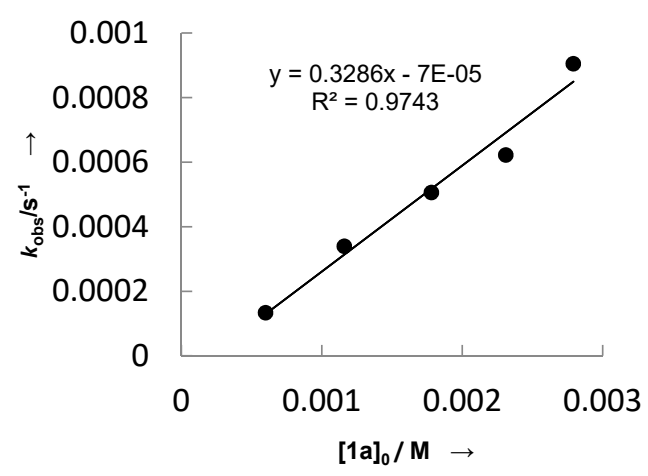

Table S4. Kinetics of the reactions of $\mathbf{1 a}$ with $\mathbf{2} \mathbf{i}$ in $\mathrm{CH}_{3} \mathrm{CN}$ at $20^{\circ} \mathrm{C}$ (deprotonated with $1.00-1.05$ equiv. $\mathrm{KO} t \mathrm{Bu}$, stopped-flow $\mathrm{UV}-\mathrm{Vis}$ spectrometer, $\lambda=540 \mathrm{~nm}$ ).

\begin{tabular}{ccc}
{$[\mathbf{2} \mathbf{i}] / \mathbf{M}$} & {$[\mathbf{1} \mathbf{a}] / \mathbf{M}$} & $\boldsymbol{k}_{\text {obs }} / \mathbf{s}^{-\mathbf{1}}$ \\
$2.50 \times 10^{-5}$ & $2.76 \times 10^{-4}$ & $7.04 \times 10^{-2}$ \\
$2.50 \times 10^{-5}$ & $4.13 \times 10^{-4}$ & $9.88 \times 10^{-2}$ \\
$2.50 \times 10^{-5}$ & $5.51 \times 10^{-4}$ & $1.30 \times 10^{-1}$ \\
$2.50 \times 10^{-5}$ & $6.89 \times 10^{-4}$ & $1.55 \times 10^{-1}$ \\
$2.50 \times 10^{-5}$ & $8.27 \times 10^{-4}$ & $2.04 \times 10^{-1}$ \\
\hline \multicolumn{3}{c}{$\left.\boldsymbol{k}_{\mathbf{2}}=\mathbf{( 2 . 3 5} \pm \mathbf{0 . 1 6}\right) \times \mathbf{1 0}^{\mathbf{2}} \mathbf{M}^{-\mathbf{1}} \mathbf{s}^{\mathbf{- 1}}$}
\end{tabular}

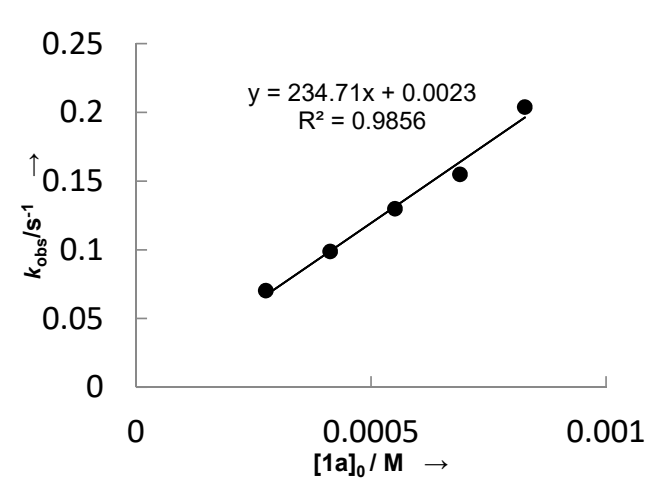


Table S5. Kinetics of the reactions of $\mathbf{1 a}$ with $\mathbf{2} \mathbf{k}$ in $\mathrm{CH}_{3} \mathrm{CN}$ at $20^{\circ} \mathrm{C}$ (deprotonated with $1.00-1.05$ equiv. $\mathrm{KO} t \mathrm{Bu}$, conventional $\mathrm{UV}-\mathrm{V}$ is spectrometer, $\lambda=527 \mathrm{~nm}$ ).

\begin{tabular}{ccc}
{$[\mathbf{2 k}] / \mathbf{M}$} & {$[\mathbf{1 a}] / \mathbf{M}$} & $\boldsymbol{k}_{\mathbf{o b s}} / \mathbf{s}^{-1}$ \\
$5.00 \times 10^{-5}$ & $4.70 \times 10^{-4}$ & $1.44 \times 10^{-1}$ \\
$5.00 \times 10^{-5}$ & $6.99 \times 10^{-4}$ & $2.14 \times 10^{-1}$ \\
$5.00 \times 10^{-5}$ & $9.33 \times 10^{-4}$ & $2.83 \times 10^{-1}$ \\
$5.00 \times 10^{-5}$ & $1.16 \times 10^{-3}$ & $3.43 \times 10^{-1}$ \\
$5.00 \times 10^{-5}$ & $1.38 \times 10^{-3}$ & $4.15 \times 10^{-1}$ \\
\hline \multicolumn{2}{c}{$\left.\boldsymbol{k}_{\mathbf{2}}=\mathbf{( 2 . 9 4} \pm \mathbf{0 . 0 5}\right) \times \mathbf{1 0}^{\mathbf{2}} \mathbf{M}^{-1} \mathbf{s}^{-1}$}
\end{tabular}

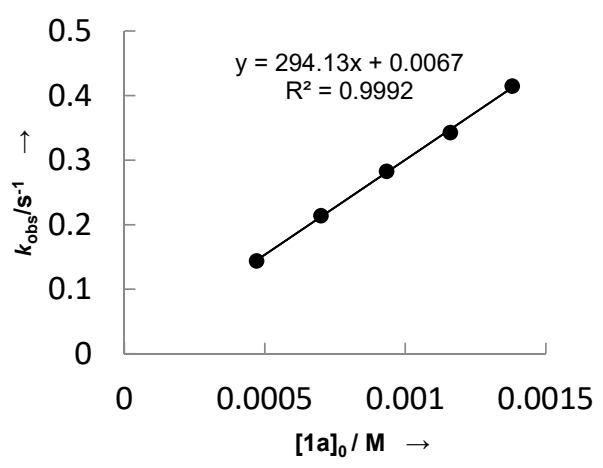

Table S6. Kinetics of the reactions of $\mathbf{1 b}$ with $\mathbf{3 a}$ in $\mathrm{CH}_{3} \mathrm{CN}$ at $20^{\circ} \mathrm{C}$ (stopped-flow UV-Vis spectrometer, $\lambda=317 \mathrm{~nm}$ ).

\begin{tabular}{ccc}
{$[\mathbf{3 a}] / \mathbf{M}$} & {$[\mathbf{1 b}] / \mathbf{M}$} & $\boldsymbol{k}_{\mathbf{0 b s}} / \mathbf{s}^{\mathbf{- 1}}$ \\
$5.00 \times 10^{-5}$ & $4.96 \times 10^{-4}$ & 1.08 \\
$5.00 \times 10^{-5}$ & $7.54 \times 10^{-4}$ & 1.73 \\
$5.00 \times 10^{-5}$ & $9.92 \times 10^{-4}$ & 2.15 \\
$5.00 \times 10^{-5}$ & $1.25 \times 10^{-3}$ & 2.66 \\
$5.00 \times 10^{-5}$ & $1.49 \times 10^{-3}$ & 3.19 \\
\hline \multicolumn{2}{c}{$\left.\boldsymbol{k}_{\mathbf{2}}=\mathbf{( 2 . 0 7} \pm \mathbf{0 . 0 7}\right) \times \mathbf{1 0}^{\mathbf{3}} \mathbf{M}^{-\mathbf{1}} \mathbf{s}^{-\mathbf{1}}$}
\end{tabular}

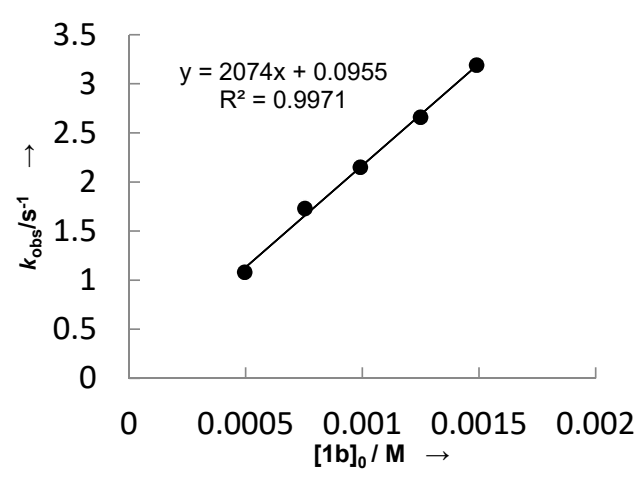

Table S7. Kinetics of the reactions of $\mathbf{1 b}$ with $\mathbf{3 b}$ in $\mathrm{CH}_{3} \mathrm{CN}$ at $20{ }^{\circ} \mathrm{C}$ (stopped-flow UV-Vis spectrometer, $\lambda=465 \mathrm{~nm}$ ).

\begin{tabular}{ccc}
{$[\mathbf{3 b}] / \mathbf{M}$} & {$[\mathbf{1 b}] / \mathbf{M}$} & $\boldsymbol{k}_{\mathbf{o b s}} / \mathbf{s}^{-1}$ \\
$5.00 \times 10^{-5}$ & $4.71 \times 10^{-4}$ & $2.20 \times 10^{-1}$ \\
$5.00 \times 10^{-5}$ & $7.16 \times 10^{-4}$ & $2.66 \times 10^{-1}$ \\
$5.00 \times 10^{-5}$ & $9.42 \times 10^{-4}$ & $3.14 \times 10^{-1}$ \\
$5.00 \times 10^{-5}$ & $1.19 \times 10^{-3}$ & $3.48 \times 10^{-1}$ \\
$5.00 \times 10^{-5}$ & $1.41 \times 10^{-3}$ & $3.91 \times 10^{-1}$ \\
\hline \multicolumn{3}{c}{$\left.\boldsymbol{k}_{\mathbf{2}}=\mathbf{( 1 . 8 0} \pm \mathbf{0 . 0 6}\right) \times \mathbf{1 0}^{\mathbf{2}} \mathbf{M}^{-1} \mathbf{s}^{-\mathbf{1}}$}
\end{tabular}

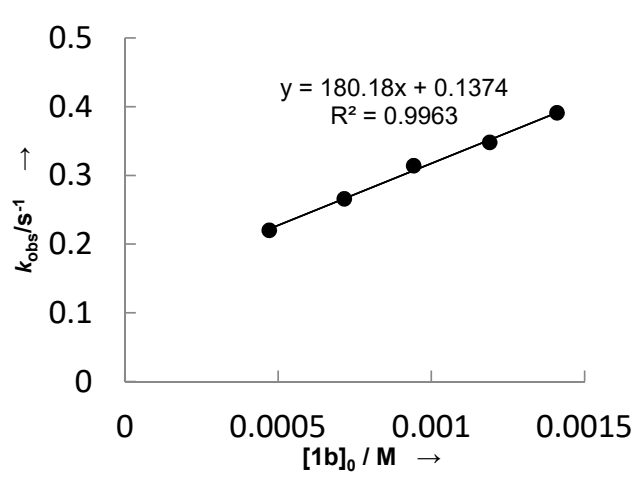

Table S8. Kinetics of the reactions of $\mathbf{1 b}$ with $\mathbf{3 c}$ in $\mathrm{CH}_{3} \mathrm{CN}$ at $20^{\circ} \mathrm{C}$ (stopped-flow UV-Vis spectrometer, $\lambda=316 \mathrm{~nm}$ ).

\begin{tabular}{ccc}
{$[\mathbf{3 c}] / \mathbf{M}$} & {$[\mathbf{1 b}] / \mathbf{M}$} & $\boldsymbol{k}_{\mathbf{o b s}} / \mathbf{s}^{-\mathbf{1}}$ \\
$5.00 \times 10^{-5}$ & $4.96 \times 10^{-4}$ & $5.01 \times 10^{-2}$ \\
$5.00 \times 10^{-5}$ & $7.54 \times 10^{-4}$ & $6.59 \times 10^{-2}$ \\
$5.00 \times 10^{-5}$ & $9.92 \times 10^{-4}$ & $8.20 \times 10^{-2}$ \\
$5.00 \times 10^{-5}$ & $1.25 \times 10^{-3}$ & $1.02 \times 10^{-1}$ \\
$5.00 \times 10^{-5}$ & $1.49 \times 10^{-3}$ & $1.26 \times 10^{-1}$ \\
\hline \multicolumn{3}{c}{$\left.\boldsymbol{k}_{\mathbf{2}}=\mathbf{( 7 . 5 6} \pm \mathbf{0 . 4 3}\right) \times \mathbf{1 0}^{\mathbf{1}} \mathbf{M}^{-\mathbf{1}} \mathbf{s}^{\mathbf{1}}$}
\end{tabular}

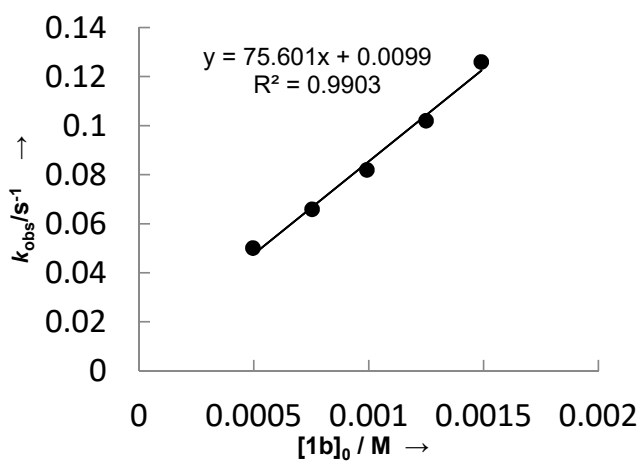


Table S9. Kinetics of the reactions of $\mathbf{1 b}$ with $\mathbf{3 d}$ in $\mathrm{CH}_{3} \mathrm{CN}$ at $20{ }^{\circ} \mathrm{C}$ (conventional UV-Vis spectrometer, $\lambda=306 \mathrm{~nm}$ ).

\begin{tabular}{|c|c|c|}
\hline$[\mathbf{3 d}] / \mathbf{M}$ & {$[1 b] / M$} & $k_{\mathrm{obs}} / \mathrm{s}^{-1}$ \\
\hline $1.00 \times 10^{-4}$ & $8.33 \times 10^{-4}$ & $6.73 \times 10^{-3}$ \\
\hline $1.00 \times 10^{-4}$ & $1.21 \times 10^{-3}$ & $8.72 \times 10^{-3}$ \\
\hline $1.00 \times 10^{-4}$ & $1.58 \times 10^{-3}$ & $1.18 \times 10^{-2}$ \\
\hline $1.00 \times 10^{-4}$ & $1.91 \times 10^{-3}$ & $1.53 \times 10^{-2}$ \\
\hline
\end{tabular}

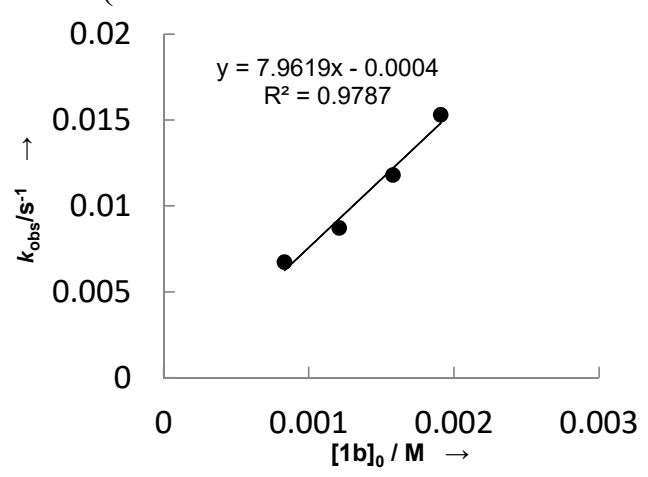

Table S10. Kinetics of the reactions of $1 \mathbf{c}$ with $2 \mathbf{a}$ in DMSO at $20^{\circ} \mathrm{C}$ (deprotonated with $1.00-1.05$ equiv. $\mathrm{KO} t \mathrm{Bu}$, conventional $\mathrm{UV}-\mathrm{Vis}$ spectrometer, $\lambda=300 \mathrm{~nm}$ ).

\begin{tabular}{|c|c|c|}
\hline$[2 \mathbf{a}] / \mathbf{M}$ & {$[1 \mathrm{c}] / \mathbf{M}$} & $k_{\mathrm{obs}} / \mathrm{s}^{-1}$ \\
\hline $5.00 \times 10^{-5}$ & $6.43 \times 10^{-4}$ & $1.58 \times 10^{-3}$ \\
\hline $5.00 \times 10^{-5}$ & $9.55 \times 10^{-4}$ & $2.30 \times 10^{-3}$ \\
\hline $5.00 \times 10^{-5}$ & $1.26 \times 10^{-3}$ & $2.77 \times 10^{-3}$ \\
\hline $5.00 \times 10^{-5}$ & $1.56 \times 10^{-3}$ & $3.53 \times 10^{-3}$ \\
\hline $5.00 \times 10^{-5}$ & $1.85 \times 10^{-3}$ & $4.03 \times 10^{-3}$ \\
\hline
\end{tabular}

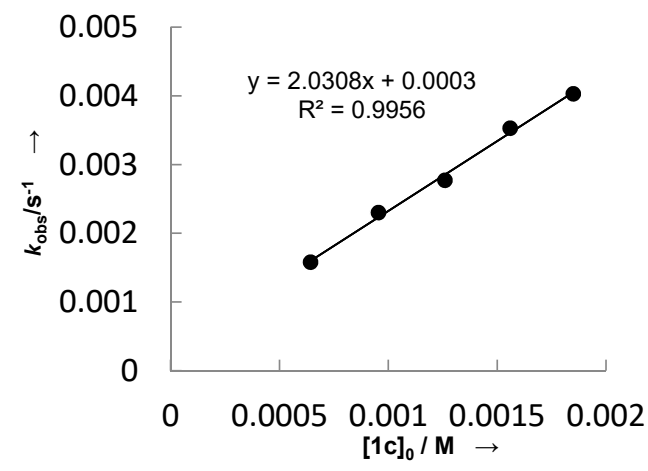

Table S11. Kinetics of the reactions of $\mathbf{1 c}$ with $2 \mathrm{l}$ in DMSO at $20^{\circ} \mathrm{C}$ (deprotonated with 0.50 equiv. $\mathrm{KO} t \mathrm{Bu}$, conventional $\mathrm{UV}$-Vis spectrometer, $\lambda=326 \mathrm{~nm}$, exponential increase).

\begin{tabular}{ccc}
{$[\mathbf{1} \mathbf{c}] / \mathbf{M}$} & {$[\mathbf{2 1}] / \mathbf{M}$} & $\boldsymbol{k}_{\mathbf{o b s}} / \mathbf{s}^{-1}$ \\
$5.00 \times 10^{-5}$ & $5.30 \times 10^{-4}$ & $1.89 \times 10^{-2}$ \\
$5.00 \times 10^{-5}$ & $7.86 \times 10^{-4}$ & $3.21 \times 10^{-2}$ \\
$5.00 \times 10^{-5}$ & $1.04 \times 10^{-3}$ & $4.72 \times 10^{-2}$ \\
$5.00 \times 10^{-5}$ & $1.28 \times 10^{-3}$ & $6.03 \times 10^{-2}$ \\
$5.00 \times 10^{-5}$ & $1.53 \times 10^{-3}$ & $7.06 \times 10^{-2}$ \\
\hline \multicolumn{2}{c}{$\left.\boldsymbol{k}_{\mathbf{2}}=\mathbf{( 5 . 2 8} \pm \mathbf{0 . 1 8}\right) \times \mathbf{1 0}^{\mathbf{1}} \mathbf{M}^{-\mathbf{1}} \mathbf{s}^{-1}$}
\end{tabular}

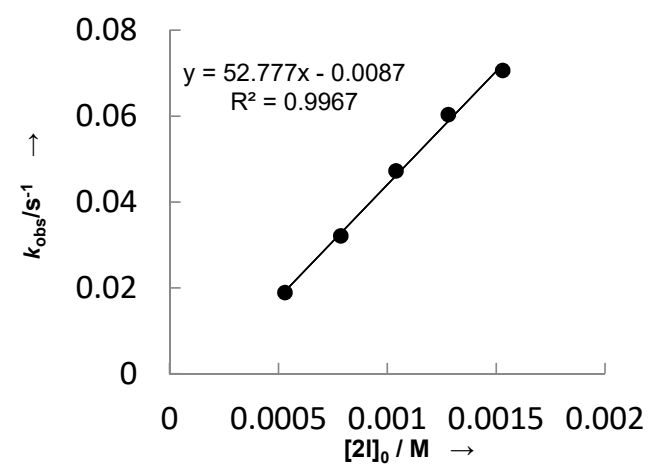

Table S12. Kinetics of the reactions of $\mathbf{1 c}$ with $\mathbf{2 n}$ in DMSO at $20{ }^{\circ} \mathrm{C}$ (deprotonated with 0.50 equiv. $\mathrm{KO} t \mathrm{Bu}$, conventional UV-Vis spectrometer, $\lambda=320 \mathrm{~nm}$, exponential increase).

\begin{tabular}{ccc}
{$[\mathbf{1} \mathbf{c}] / \mathbf{M}$} & {$[\mathbf{2 n}] / \mathbf{M}$} & $\boldsymbol{k}_{\mathbf{o b s}} / \mathbf{s}^{-\mathbf{1}}$ \\
$5.00 \times 10^{-5}$ & $5.07 \times 10^{-4}$ & $1.18 \times 10^{-2}$ \\
$5.00 \times 10^{-5}$ & $7.53 \times 10^{-4}$ & $1.82 \times 10^{-2}$ \\
$5.00 \times 10^{-5}$ & $1.01 \times 10^{-3}$ & $2.48 \times 10^{-2}$ \\
$5.00 \times 10^{-5}$ & $1.25 \times 10^{-3}$ & $3.15 \times 10^{-2}$ \\
$5.00 \times 10^{-5}$ & $1.49 \times 10^{-3}$ & $3.83 \times 10^{-2}$ \\
\hline \multicolumn{3}{c}{$\left.\boldsymbol{k}_{\mathbf{2}}=\mathbf{( 2 . 6 9} \pm \mathbf{0 . 0 4}\right) \times \mathbf{1 0}^{\mathbf{1}} \mathbf{M}^{-\mathbf{1}} \mathbf{s}^{-\mathbf{1}}$}
\end{tabular}

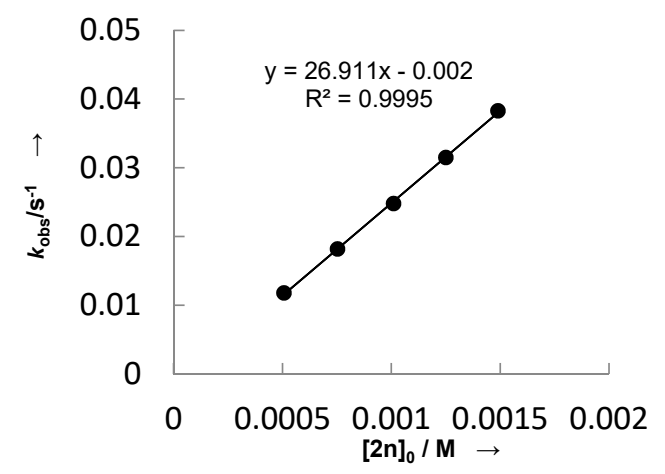


Table S13. Kinetics of the reactions of $1 \mathbf{c}$ with $2 \mathrm{o}$ in DMSO at $20^{\circ} \mathrm{C}$ (deprotonated with 0.50 equiv. $\mathrm{KO} t \mathrm{Bu}$, conventional $\mathrm{UV}$-Vis spectrometer, $\lambda=350 \mathrm{~nm}$, exponential increase).

\begin{tabular}{|c|c|c|}
\hline$[1 \mathrm{c}] / \mathbf{M}$ & {$[20] / \mathbf{M}$} & $k_{\text {obs }} / \mathbf{s}^{-1}$ \\
\hline $5.00 \times 10^{-5}$ & $9.86 \times 10^{-4}$ & $1.13 \times 10^{-3}$ \\
\hline $5.00 \times 10^{-5}$ & $1.21 \times 10^{-3}$ & $1.40 \times 10^{-3}$ \\
\hline $5.00 \times 10^{-5}$ & $1.45 \times 10^{-3}$ & $1.67 \times 10^{-3}$ \\
\hline $5.00 \times 10^{-5}$ & $1.68 \times 10^{-3}$ & $1.93 \times 10^{-3}$ \\
\hline $5.00 \times 10^{-5}$ & $1.93 \times 10^{-3}$ & $2.22 \times 10^{-3}$ \\
\hline
\end{tabular}

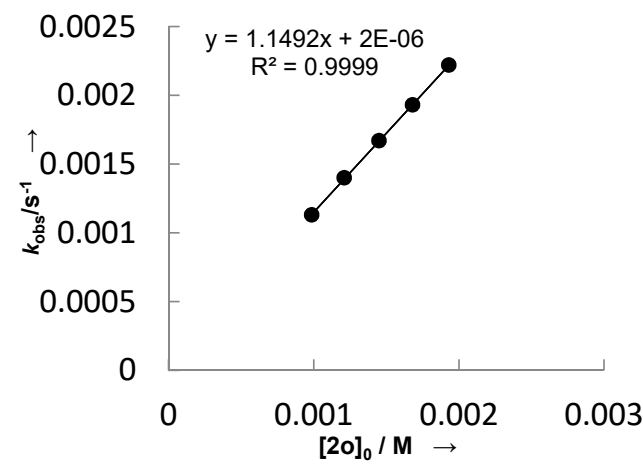

Table S14. Kinetics of the reactions of $1 \mathbf{c}$ with $\mathbf{2 p}$ in DMSO at $20^{\circ} \mathrm{C}$ (deprotonated with 0.50 equiv. $\mathrm{KO} t \mathrm{Bu}$, conventional $\mathrm{UV}$-Vis spectrometer, $\lambda=350 \mathrm{~nm}$, exponential increase).

\begin{tabular}{ccc}
{$[\mathbf{1} \mathbf{c}] / \mathbf{M}$} & {$[\mathbf{2} \mathbf{p}] / \mathbf{M}$} & $\boldsymbol{k}_{\mathbf{o b s}} / \mathbf{s}^{-1}$ \\
$5.00 \times 10^{-5}$ & $1.12 \times 10^{-3}$ & $8.21 \times 10^{-4}$ \\
$5.00 \times 10^{-5}$ & $1.41 \times 10^{-3}$ & $1.00 \times 10^{-3}$ \\
$5.00 \times 10^{-5}$ & $1.67 \times 10^{-3}$ & $1.16 \times 10^{-3}$ \\
$5.00 \times 10^{-5}$ & $1.94 \times 10^{-3}$ & $1.31 \times 10^{-3}$ \\
$5.00 \times 10^{-5}$ & $2.21 \times 10^{-3}$ & $1.54 \times 10^{-3}$ \\
\hline \multicolumn{3}{c}{$\left.\boldsymbol{k}_{\mathbf{2}}=\mathbf{( 6 . 4 5} \pm \mathbf{0 . 2 8}\right) \times \mathbf{1 0}^{-\mathbf{1}} \mathbf{M}^{-\mathbf{1}} \mathbf{s}^{-1}$}
\end{tabular}

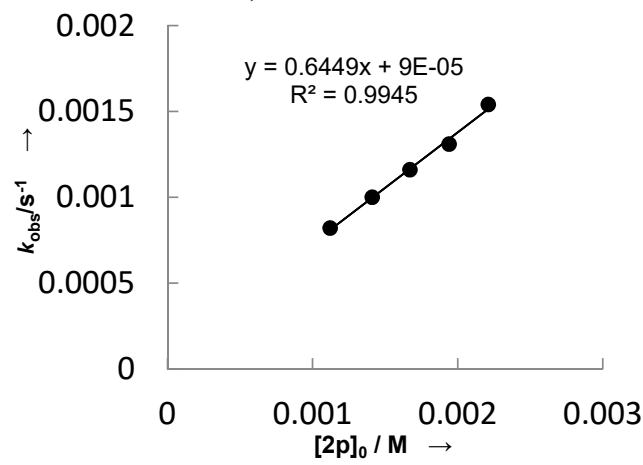

Table S15. Kinetics of the reactions of $\mathbf{1 d}$ with $\mathbf{2 c}$ in DMSO at $20^{\circ} \mathrm{C}$ (deprotonated with $1.00-1.05$ equiv. $\mathrm{KO} t \mathrm{Bu}$, conventional $\mathrm{UV}-\mathrm{V}$ is spectrometer, $\lambda=344 \mathrm{~nm}$ ).

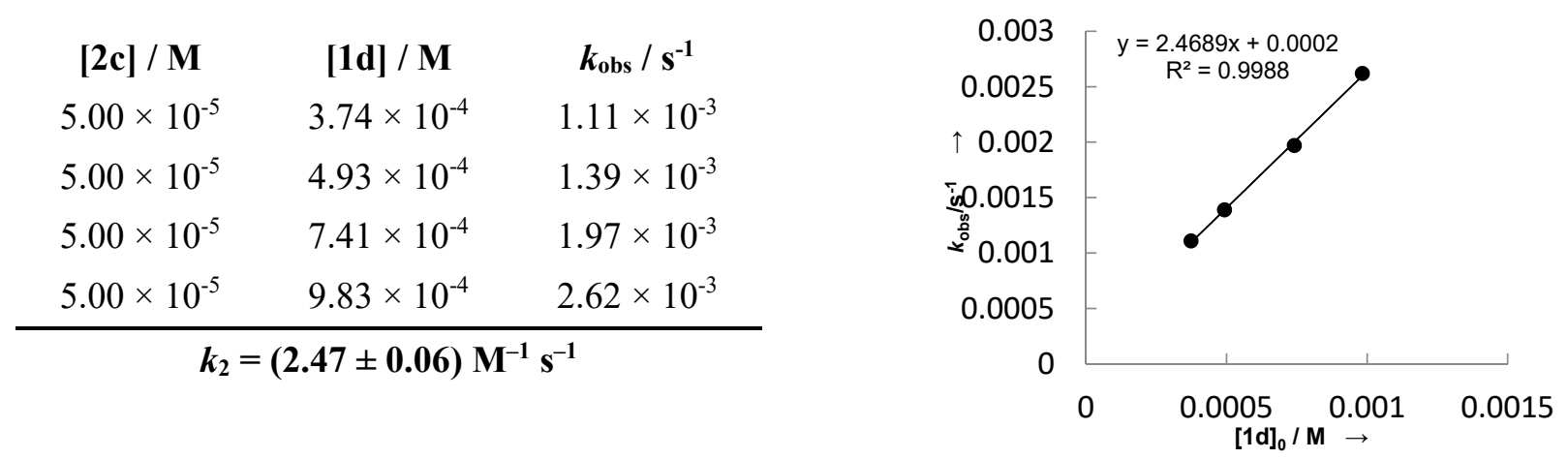

Table S16. Kinetics of the reactions of $\mathbf{1 d}$ with $\mathbf{2} \mathbf{m}$ in DMSO at $20^{\circ} \mathrm{C}$ (deprotonated with 0.50 equiv. $\mathrm{KO} t \mathrm{Bu}$, conventional UV-Vis spectrometer, $\lambda=439 \mathrm{~nm}$, exponential increase).

\begin{tabular}{|c|c|c|}
\hline$[\mathbf{1 d}] / \mathbf{M}$ & {$[2 \mathrm{~m}] / \mathrm{M}$} & $k_{\mathrm{obs}} / \mathrm{s}^{-1}$ \\
\hline $5.00 \times 10^{-5}$ & $5.31 \times 10^{-4}$ & $1.32 \times 10^{-1}$ \\
\hline $5.00 \times 10^{-5}$ & $7.97 \times 10^{-4}$ & $2.08 \times 10^{-1}$ \\
\hline $5.00 \times 10^{-5}$ & $1.06 \times 10^{-3}$ & $2.72 \times 10^{-1}$ \\
\hline $5.00 \times 10^{-5}$ & $1.33 \times 10^{-3}$ & $3.52 \times 10^{-1}$ \\
\hline $5.00 \times 10^{-5}$ & $1.59 \times 10^{-3}$ & $4.20 \times 10^{-1}$ \\
\hline
\end{tabular}

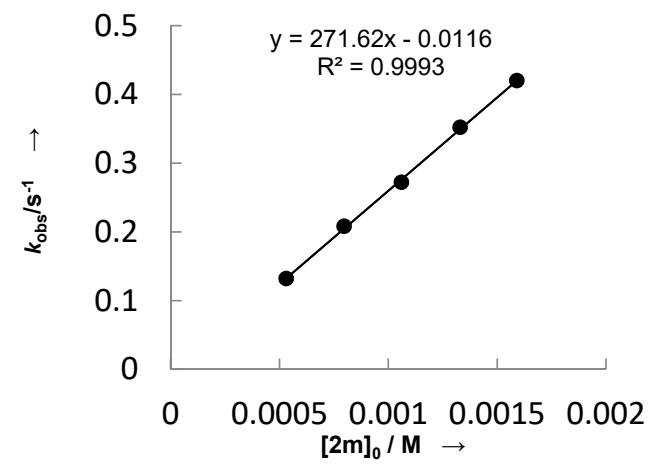


Table S17. Kinetics of the reactions of $\mathbf{1 d}$ with $\mathbf{2 n}$ in DMSO at $20^{\circ} \mathrm{C}$ (deprotonated with 0.50 equiv. $\mathrm{KO} t \mathrm{Bu}$, stopped-flow $\mathrm{UV}$-Vis spectrometer, $\lambda=414 \mathrm{~nm}$, exponential increase).

\begin{tabular}{ccc}
{$[\mathbf{1} \mathbf{d}] / \mathbf{M}$} & {$[\mathbf{2 n}] / \mathbf{M}$} & $\boldsymbol{k}_{\mathbf{o b s}} / \mathbf{s}^{-\mathbf{1}}$ \\
$1.00 \times 10^{-4}$ & $1.01 \times 10^{-3}$ & 1.13 \\
$1.00 \times 10^{-4}$ & $1.52 \times 10^{-3}$ & 1.73 \\
$1.00 \times 10^{-4}$ & $2.03 \times 10^{-3}$ & 2.35 \\
$1.00 \times 10^{-4}$ & $2.53 \times 10^{-3}$ & 3.00 \\
$1.00 \times 10^{-4}$ & $3.04 \times 10^{-3}$ & 3.64 \\
\hline \multicolumn{2}{c}{$\left.\boldsymbol{k}_{\mathbf{2}}=\mathbf{( 1 . 2 4} \pm \mathbf{0 . 0 2}\right) \times \mathbf{1 0}^{\mathbf{3}} \mathbf{M}^{-\mathbf{1}} \mathbf{s}^{-\mathbf{1}}$}
\end{tabular}

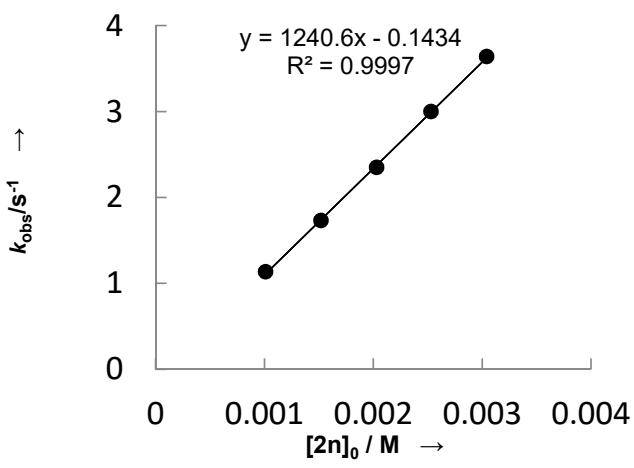

Table S18. Kinetics of the reactions of $\mathbf{1 d}$ with $2 \mathbf{o}$ in DMSO at $20^{\circ} \mathrm{C}$ (deprotonated with 0.50 equiv. $\mathrm{KO} t \mathrm{Bu}$, stopped-flow $\mathrm{UV}$-Vis spectrometer, $\lambda=426 \mathrm{~nm}$, exponential increase).

\begin{tabular}{ccc}
{$[\mathbf{1 d}] / \mathbf{M}$} & {$[\mathbf{2 o}] / \mathbf{M}$} & $\boldsymbol{k}_{\mathbf{o b s}} / \mathbf{s}^{-\mathbf{1}}$ \\
$5.00 \times 10^{-5}$ & $4.96 \times 10^{-4}$ & $3.91 \times 10^{-2}$ \\
$5.00 \times 10^{-5}$ & $7.43 \times 10^{-4}$ & $6.22 \times 10^{-2}$ \\
$5.00 \times 10^{-5}$ & $9.91 \times 10^{-4}$ & $8.24 \times 10^{-2}$ \\
$5.00 \times 10^{-5}$ & $1.24 \times 10^{-3}$ & $1.06 \times 10^{-1}$ \\
$5.00 \times 10^{-5}$ & $1.49 \times 10^{-3}$ & $1.30 \times 10^{-1}$ \\
\hline \multicolumn{3}{c}{$\left.\boldsymbol{k}_{\mathbf{2}}=\mathbf{( 9 . 0 8} \pm \mathbf{0 . 1 4}\right) \times \mathbf{1 0}^{\mathbf{1}} \mathbf{M}^{-\mathbf{1}} \mathbf{s}^{-\mathbf{1}}$}
\end{tabular}

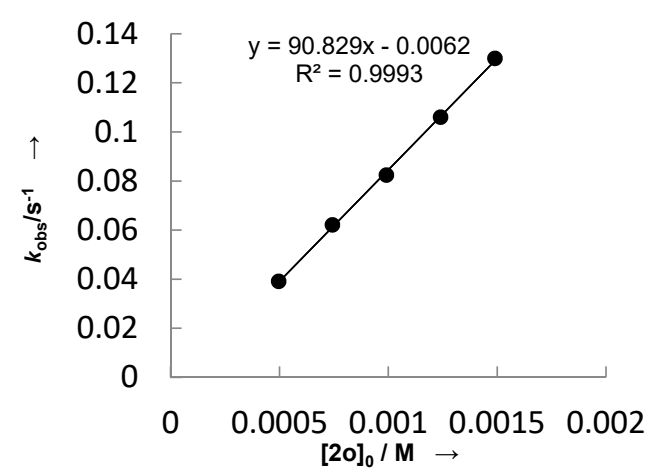

Table S19. Kinetics of the reactions of $\mathbf{1 d}$ with $\mathbf{2 p}$ in DMSO at $20^{\circ} \mathrm{C}$ (deprotonated with 0.50 equiv. $\mathrm{KO} t \mathrm{Bu}$, conventional $\mathrm{UV}-\mathrm{Vis}$ spectrometer, $\lambda=402 \mathrm{~nm}$, exponential increase).

\begin{tabular}{ccc}
{$[\mathbf{1} \mathbf{d}] / \mathbf{M}$} & {$[\mathbf{2} \mathbf{p}] / \mathbf{M}$} & $\boldsymbol{k}_{\mathbf{0 b s}} / \mathbf{s}^{-\mathbf{1}}$ \\
$1.00 \times 10^{-4}$ & $9.68 \times 10^{-4}$ & $1.32 \times 10^{-2}$ \\
$1.00 \times 10^{-4}$ & $1.47 \times 10^{-3}$ & $1.98 \times 10^{-2}$ \\
$1.00 \times 10^{-4}$ & $1.92 \times 10^{-3}$ & $2.01 \times 10^{-2}$ \\
$1.00 \times 10^{-4}$ & $2.40 \times 10^{-3}$ & $2.39 \times 10^{-2}$ \\
$1.00 \times 10^{-4}$ & $2.70 \times 10^{-3}$ & $2.79 \times 10^{-2}$ \\
\hline \multicolumn{3}{c}{$\left.\boldsymbol{k}_{\mathbf{2}}=\mathbf{( 7 . 5 8} \pm \mathbf{1 . 1 2}\right) \mathbf{M}^{-\mathbf{1}} \mathbf{s}^{-1}$}
\end{tabular}

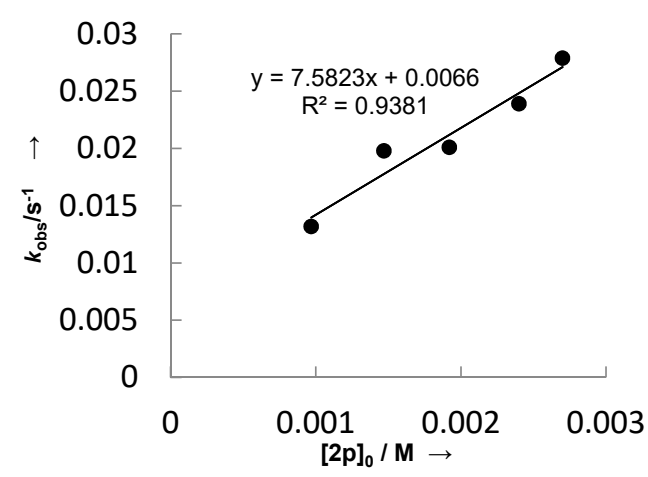

Table S20. Kinetics of the reactions of 1e with 2a in DMSO at $20^{\circ} \mathrm{C}$ (deprotonated with $1.00-1.05$ equiv. $\mathrm{KO} t \mathrm{Bu}$, conventional $\mathrm{UV}$-Vis spectrometer, $\lambda=320 \mathrm{~nm}$, exponential increase).

\begin{tabular}{ccc}
{$[\mathbf{2 a}] / \mathbf{M}$} & {$[\mathbf{1 e}] / \mathbf{M}$} & $\boldsymbol{k}_{\mathbf{0 b s}} / \mathbf{s}^{-1}$ \\
$5.00 \times 10^{-5}$ & $5.50 \times 10^{-4}$ & $2.34 \times 10^{-4}$ \\
$5.00 \times 10^{-5}$ & $8.13 \times 10^{-4}$ & $3.13 \times 10^{-4}$ \\
$5.00 \times 10^{-5}$ & $1.07 \times 10^{-3}$ & $3.73 \times 10^{-4}$ \\
$5.00 \times 10^{-5}$ & $1.32 \times 10^{-3}$ & $4.31 \times 10^{-4}$ \\
$5.00 \times 10^{-5}$ & $1.57 \times 10^{-3}$ & $5.32 \times 10^{-4}$ \\
\hline \multicolumn{3}{c}{$\boldsymbol{k}_{\mathbf{2}}=\mathbf{( 2 . 8 0 \pm 0 . 1 7 )} \times \mathbf{1 0}^{-\mathbf{1}} \mathbf{M}^{-\mathbf{1}} \mathbf{s}^{-1}$}
\end{tabular}

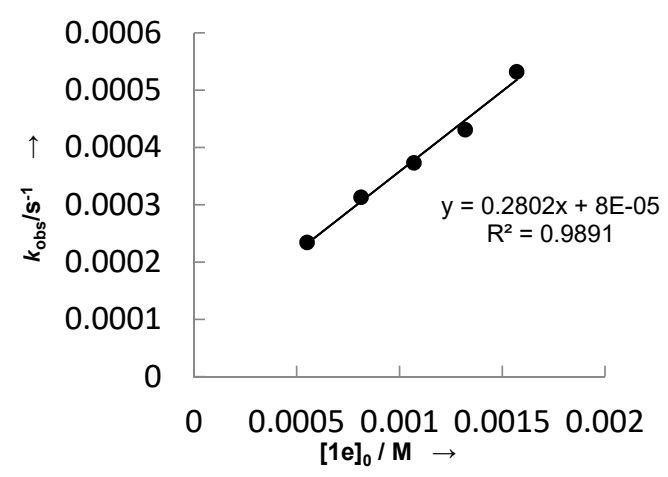


Table S21. Kinetics of the reactions of $\mathbf{1 e}$ with $\mathbf{2 f}$ in DMSO at $20^{\circ} \mathrm{C}$ (deprotonated with $1.00-1.05$ equiv. $\mathrm{KO} t \mathrm{Bu}$, stopped-flow $\mathrm{UV}$-Vis spectrometer, $\lambda=405 \mathrm{~nm}$ ).

\begin{tabular}{ccc}
$\mathbf{2 f}] / \mathbf{M}$ & {$[\mathbf{1 e}] / \mathbf{M}$} & $\boldsymbol{k}_{\mathbf{o b s}} / \mathbf{s}^{\mathbf{- 1}}$ \\
$2.50 \times 10^{-5}$ & $2.63 \times 10^{-4}$ & $8.36 \times 10^{-2}$ \\
$2.50 \times 10^{-5}$ & $4.00 \times 10^{-4}$ & $1.16 \times 10^{-1}$ \\
$2.50 \times 10^{-5}$ & $5.26 \times 10^{-4}$ & $1.22 \times 10^{-1}$ \\
$2.50 \times 10^{-5}$ & $6.62 \times 10^{-4}$ & $1.37 \times 10^{-1}$ \\
$2.50 \times 10^{-5}$ & $7.89 \times 10^{-4}$ & $1.66 \times 10^{-1}$ \\
\hline \multicolumn{2}{c}{$\left.\boldsymbol{k}_{\mathbf{2}}=\mathbf{( 1 . 4 2} \pm \mathbf{0 . 1 8}\right) \times \mathbf{1 0}^{\mathbf{2}} \mathbf{M}^{-\mathbf{1}} \mathbf{s}^{\mathbf{- 1}}$}
\end{tabular}

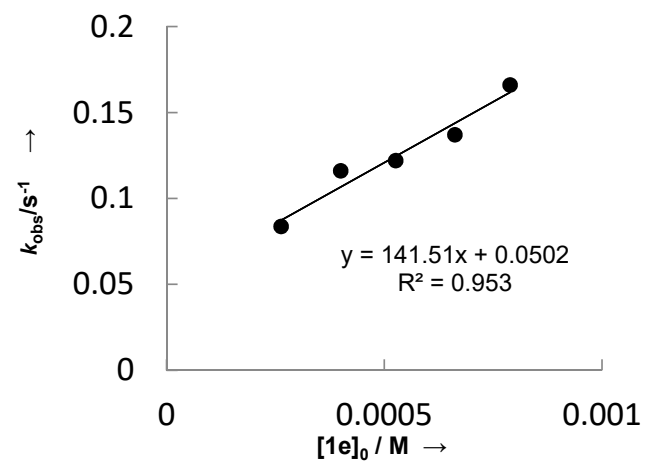

Table S22. Kinetics of the reactions of $\mathbf{1 e}$ with $\mathbf{2 h}$ in DMSO at $20^{\circ} \mathrm{C}$ (deprotonated with $1.00-1.05$ equiv. $\mathrm{KO} t \mathrm{Bu}$, stopped-flow $\mathrm{UV}-\mathrm{Vis}$ spectrometer, $\lambda=395 \mathrm{~nm}$ ).

\begin{tabular}{ccc}
{$[\mathbf{2 h}] / \mathbf{M}$} & {$[\mathbf{1} \mathbf{e}] / \mathbf{M}$} & $\boldsymbol{k}_{\mathbf{o b s}} / \mathbf{s}^{-1}$ \\
$5.00 \times 10^{-5}$ & $5.13 \times 10^{-4}$ & $9.27 \times 10^{-2}$ \\
$5.00 \times 10^{-5}$ & $7.70 \times 10^{-4}$ & $1.32 \times 10^{-1}$ \\
$5.00 \times 10^{-5}$ & $8.98 \times 10^{-4}$ & $1.58 \times 10^{-1}$ \\
$5.00 \times 10^{-5}$ & $1.28 \times 10^{-3}$ & $2.21 \times 10^{-1}$ \\
\hline \multicolumn{3}{c}{$\left.\boldsymbol{k}_{\mathbf{2}}=\mathbf{( 1 . 6 9} \pm \mathbf{0 . 0 5}\right) \times \mathbf{1 0}^{\mathbf{2}} \mathbf{M}^{-1} \mathbf{s}^{-\mathbf{1}}$}
\end{tabular}

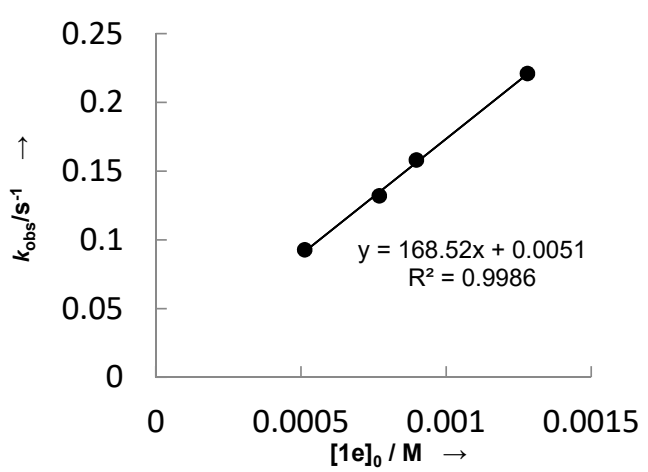

Table S23. Kinetics of the reactions of $1 \mathbf{e}$ with $\mathbf{2 m}$ in DMSO at $20^{\circ} \mathrm{C}$ (deprotonated with 0.50 equiv. $\mathrm{KO} t \mathrm{Bu}$, conventional UV-Vis spectrometer, $\lambda=295 \mathrm{~nm}$, exponential increase).

\begin{tabular}{ccc}
{$[\mathbf{1} \mathbf{e}] / \mathbf{M}$} & {$[\mathbf{2} \mathbf{m}] / \mathbf{M}$} & $\boldsymbol{k}_{\mathbf{o b s}} / \mathbf{s}^{-1}$ \\
$5.00 \times 10^{-5}$ & $5.26 \times 10^{-4}$ & $4.04 \times 10^{-4}$ \\
$5.00 \times 10^{-5}$ & $7.95 \times 10^{-4}$ & $5.13 \times 10^{-4}$ \\
$5.00 \times 10^{-5}$ & $1.04 \times 10^{-3}$ & $6.44 \times 10^{-4}$ \\
$5.00 \times 10^{-5}$ & $1.31 \times 10^{-3}$ & $8.50 \times 10^{-4}$ \\
$5.00 \times 10^{-5}$ & $1.55 \times 10^{-3}$ & $1.00 \times 10^{-3}$ \\
\hline \multicolumn{3}{c}{$\left.\boldsymbol{k}_{\mathbf{2}}=\mathbf{( 5 . 9 6} \pm \mathbf{0 . 3 9}\right) \times \mathbf{1 0}^{-\mathbf{1}} \mathbf{M}^{-\mathbf{1}} \mathbf{s}^{\mathbf{- 1}}$}
\end{tabular}

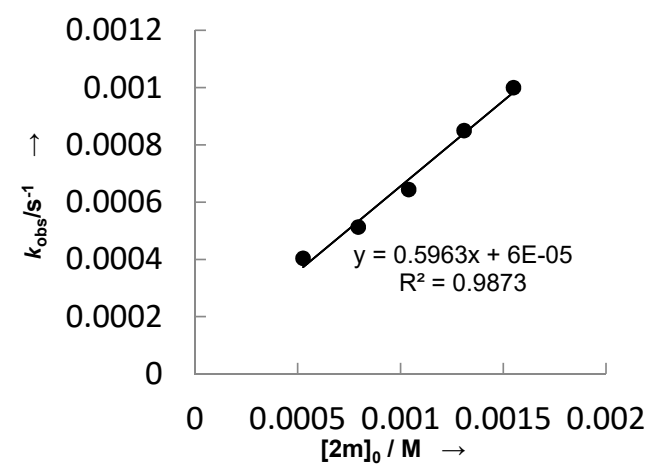

Table S24. Kinetics of the reactions of $1 \mathbf{e}$ with 20 in DMSO at $20^{\circ} \mathrm{C}$ (deprotonated with 0.50 equiv. $\mathrm{KO} t \mathrm{Bu}$, conventional UV-Vis spectrometer, $\lambda=315 \mathrm{~nm}$, exponential increase).

\begin{tabular}{|c|c|c|c|c|}
\hline$[1 \mathrm{e}] / \mathbf{M}$ & {$[20] / M$} & $k_{\text {obs }} / \mathbf{s}^{-1}$ & 0.0006 & \\
\hline $5.00 \times 10^{-5}$ & $1.08 \times 10^{-3}$ & $2.84 \times 10^{-4}$ & $\begin{array}{r}0.0005 \\
\uparrow \quad 0.0004\end{array}$ & \\
\hline $5.00 \times 10^{-5}$ & $1.35 \times 10^{-3}$ & $3.58 \times 10^{-4}$ & is 0.0003 & \\
\hline $5.00 \times 10^{-5}$ & $1.59 \times 10^{-3}$ & $4.02 \times 10^{-4}$ & 象 0.0002 & $\begin{array}{l}y=0.2409 x+3 E-05 \\
R^{2}=0.9843\end{array}$ \\
\hline $\begin{array}{l}5.00 \times 10^{-5} \\
5.00 \times 10^{-5}\end{array}$ & $\begin{array}{l}1.85 \times 10^{-3} \\
2.09 \times 10^{-3}\end{array}$ & $\begin{array}{l}4.92 \times 10^{-4} \\
5.20 \times 10^{-4}\end{array}$ & 0.0001 & \\
\hline$k_{2}=$ & 10 & & 0 & $\underset{[20]_{0} / \mathrm{M}}{0.001} \stackrel{0.002}{\rightarrow}$ \\
\hline
\end{tabular}


Table S25. Kinetics of the reactions of $1 \mathbf{g}$ with 2a in DMSO at $20^{\circ} \mathrm{C}$ (deprotonated with $1.00-1.05$ equiv. $\mathrm{KO} t \mathrm{Bu}$, conventional $\mathrm{UV}-\mathrm{Vis}$ spectrometer, $\lambda=295 \mathrm{~nm}$ ).

\begin{tabular}{ccc}
{$[\mathbf{2 a}] / \mathbf{M}$} & {$[\mathbf{1 g}] / \mathbf{M}$} & $\boldsymbol{k}_{\mathbf{o b s}} / \mathbf{s}^{-\mathbf{1}}$ \\
$5.00 \times 10^{-5}$ & $4.98 \times 10^{-4}$ & $4.46 \times 10^{-3}$ \\
$5.00 \times 10^{-5}$ & $7.36 \times 10^{-4}$ & $6.62 \times 10^{-3}$ \\
$5.00 \times 10^{-5}$ & $9.77 \times 10^{-4}$ & $9.05 \times 10^{-3}$ \\
$5.00 \times 10^{-5}$ & $1.21 \times 10^{-3}$ & $1.14 \times 10^{-2}$ \\
$5.00 \times 10^{-5}$ & $1.45 \times 10^{-3}$ & $1.40 \times 10^{-2}$ \\
\hline \multicolumn{3}{c}{$\left.\boldsymbol{k}_{\mathbf{2}}=\mathbf{( 1 . 0 0} \pm \mathbf{0 . 0 2}\right) \times \mathbf{1 0}^{\mathbf{1}} \mathbf{M}^{-\mathbf{1}} \mathbf{s}^{-\mathbf{1}}$}
\end{tabular}

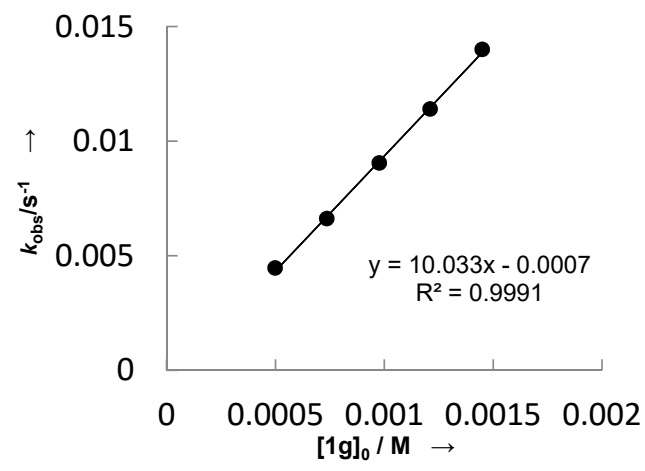

Table S26. Kinetics of the reactions of $\mathbf{1 g}$ with $\mathbf{2 b}$ in DMSO at $20^{\circ} \mathrm{C}$ (deprotonated with $1.00-1.05$ equiv. $\mathrm{KO} t \mathrm{Bu}$, conventional $\mathrm{UV}-\mathrm{Vis}$ spectrometer, $\lambda=320 \mathrm{~nm}$ ).

\begin{tabular}{|c|c|c|}
\hline$[2 \mathrm{~b}] / \mathbf{M}$ & {$[1 \mathrm{~g}] / \mathrm{M}$} & $k_{\mathrm{obs}} / \mathrm{s}^{-1}$ \\
\hline $5.00 \times 10^{-5}$ & $4.84 \times 10^{-4}$ & $1.98 \times 10^{-4}$ \\
\hline $5.00 \times 10^{-5}$ & $7.21 \times 10^{-4}$ & $3.34 \times 10^{-4}$ \\
\hline $5.00 \times 10^{-5}$ & $9.52 \times 10^{-4}$ & $4.04 \times 10^{-4}$ \\
\hline $5.00 \times 10^{-5}$ & $1.18 \times 10^{-3}$ & $4.85 \times 10^{-4}$ \\
\hline $5.00 \times 10^{-5}$ & $1.42 \times 10^{-3}$ & $6.10 \times 10^{-4}$ \\
\hline
\end{tabular}

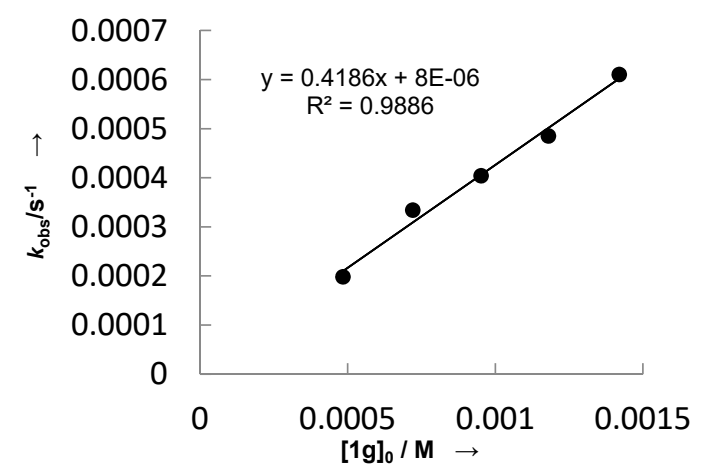

Table S27. Kinetics of the reactions of $\mathbf{1 g}$ with $\mathbf{2} \mathbf{f}$ in DMSO at $20^{\circ} \mathrm{C}$ (deprotonated with $1.00-1.05$ equiv. $\mathrm{KO} t \mathrm{Bu}$, stopped-flow $\mathrm{UV}-\mathrm{Vis}$ spectrometer, $\lambda=405 \mathrm{~nm}$ ).

\begin{tabular}{ccc}
{$[\mathbf{2 f}] / \mathbf{M}$} & {$[\mathbf{1 g}] / \mathbf{M}$} & $\boldsymbol{k}_{\mathbf{o b s}} / \mathbf{s}^{-\mathbf{1}}$ \\
$2.50 \times 10^{-5}$ & $2.53 \times 10^{-4}$ & $6.85 \times 10^{-1}$ \\
$2.50 \times 10^{-5}$ & $3.79 \times 10^{-4}$ & 1.09 \\
$2.50 \times 10^{-5}$ & $5.05 \times 10^{-4}$ & 1.28 \\
$2.50 \times 10^{-5}$ & $6.32 \times 10^{-3}$ & 1.41 \\
$2.50 \times 10^{-5}$ & $7.58 \times 10^{-3}$ & 1.65 \\
\hline \multicolumn{3}{c}{$\left.\boldsymbol{k}_{\mathbf{2}}=\mathbf{( 1 . 7 8} \pm \mathbf{0 . 2 1}\right) \times \mathbf{1 0}^{\mathbf{3}} \mathbf{M}^{-\mathbf{1}} \mathbf{s}^{\mathbf{1}}$}
\end{tabular}

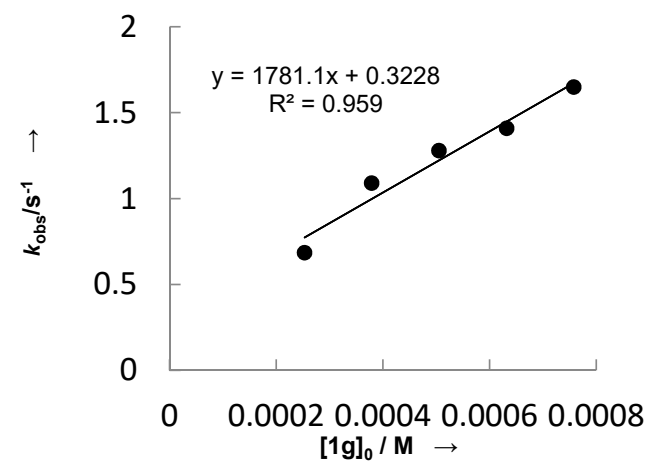

Table S28. Kinetics of the reactions of $\mathbf{1 g}$ with $\mathbf{2 g}$ in DMSO at $20^{\circ} \mathrm{C}$ (deprotonated with $1.00-1.05$ equiv. $\mathrm{KO} t \mathrm{Bu}$, stopped-flow $\mathrm{UV}$-Vis spectrometer, $\lambda=550 \mathrm{~nm}$ ).

\begin{tabular}{|c|c|c|}
\hline$[2 \mathrm{~g}] / \mathrm{M}$ & {$[1 \mathrm{~g}] / \mathrm{M}$} & $k_{\text {obs }} / \mathbf{s}^{-1}$ \\
\hline $2.50 \times 10^{-5}$ & $3.36 \times 10^{-4}$ & $9.01 \times 10^{-3}$ \\
\hline $2.50 \times 10^{-5}$ & $5.03 \times 10^{-4}$ & $1.25 \times 10^{-2}$ \\
\hline $2.50 \times 10^{-5}$ & $6.71 \times 10^{-4}$ & $1.66 \times 10^{-2}$ \\
\hline $2.50 \times 10^{-5}$ & $8.39 \times 10^{-4}$ & $1.98 \times 10^{-2}$ \\
\hline $2.50 \times 10^{-5}$ & $1.01 \times 10^{-3}$ & $2.38 \times 10^{-2}$ \\
\hline
\end{tabular}

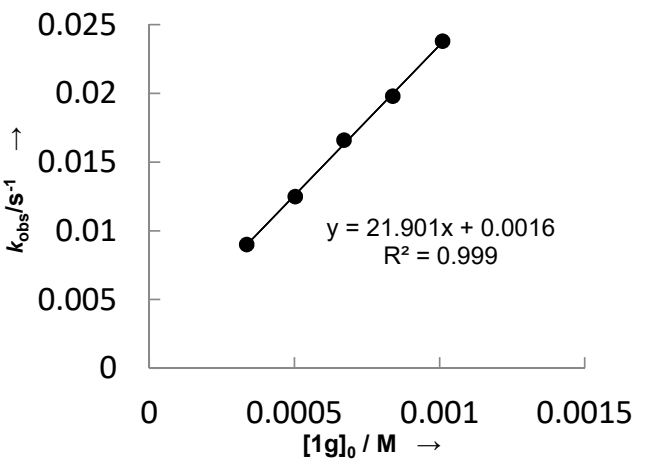


Table S29. Kinetics of the reactions of $\mathbf{1 g}$ with $\mathbf{2} \mathbf{i}$ in DMSO at $20^{\circ} \mathrm{C}$ (deprotonated with $1.00-1.05$ equiv. $\mathrm{KO} t \mathrm{Bu}$, conventional $\mathrm{UV}-\mathrm{Vis}$ spectrometer, $\lambda=540 \mathrm{~nm}$ ).

\begin{tabular}{ccc}
{$[\mathbf{2 i}] / \mathbf{M}$} & {$[\mathbf{1 g}] / \mathbf{M}$} & $\boldsymbol{k}_{\mathbf{o b s}} / \mathbf{s}^{-\mathbf{1}}$ \\
$5.00 \times 10^{-5}$ & $4.54 \times 10^{-4}$ & $1.23 \times 10^{-2}$ \\
$5.00 \times 10^{-5}$ & $6.75 \times 10^{-4}$ & $1.90 \times 10^{-2}$ \\
$5.00 \times 10^{-5}$ & $8.92 \times 10^{-4}$ & $2.45 \times 10^{-2}$ \\
$5.00 \times 10^{-5}$ & $1.10 \times 10^{-3}$ & $2.95 \times 10^{-2}$ \\
$5.00 \times 10^{-5}$ & $1.31 \times 10^{-3}$ & $3.48 \times 10^{-2}$ \\
\hline \multicolumn{3}{c}{$\left.\boldsymbol{k}_{\mathbf{2}}=\mathbf{( 2 . 6 0} \pm \mathbf{0 . 0 7}\right) \times \mathbf{1 0}^{\mathbf{1}} \mathbf{M}^{-1} \mathbf{s}^{-\mathbf{1}}$}
\end{tabular}

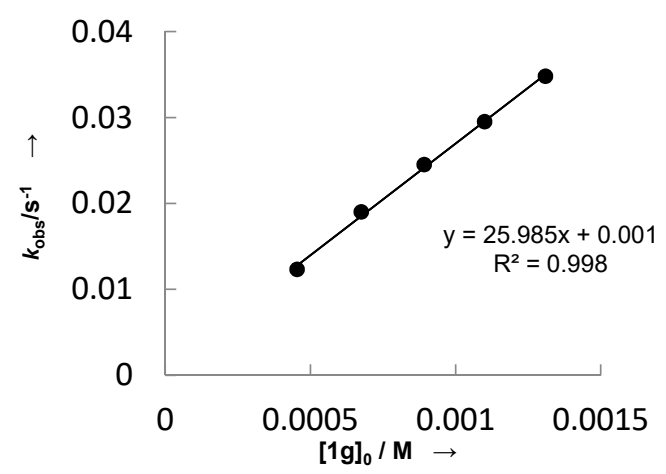

Table S30. Kinetics of the reactions of $\mathbf{1 g}$ with $\mathbf{2 \mathbf { j }}$ in DMSO at $20^{\circ} \mathrm{C}$ (deprotonated with $1.00-1.05$ equiv. $\mathrm{KO} t \mathrm{Bu}$, stopped-flow $\mathrm{UV}$-Vis spectrometer, $\lambda=265 \mathrm{~nm}$ ).

\begin{tabular}{ccc}
{$[\mathbf{2} \mathbf{j}] / \mathbf{M}$} & {$[\mathbf{1 g}] / \mathbf{M}$} & $\boldsymbol{k}_{\mathbf{o b s}} / \mathbf{s}^{-1}$ \\
$8.00 \times 10^{-5}$ & $8.66 \times 10^{-4}$ & $8.32 \times 10^{1}$ \\
$8.00 \times 10^{-5}$ & $1.30 \times 10^{-3}$ & $1.17 \times 10^{2}$ \\
$8.00 \times 10^{-5}$ & $1.73 \times 10^{-3}$ & $1.82 \times 10^{2}$ \\
$8.00 \times 10^{-5}$ & $2.60 \times 10^{-3}$ & $2.48 \times 10^{2}$ \\
\hline \multicolumn{3}{c}{$\boldsymbol{k}_{\mathbf{2}}=\mathbf{( 9 . 7 6} \pm \mathbf{0 . 9 6 )} \times \mathbf{1 0}^{\mathbf{4}} \mathbf{M}^{-1} \mathbf{s}^{-\mathbf{1}}$}
\end{tabular}

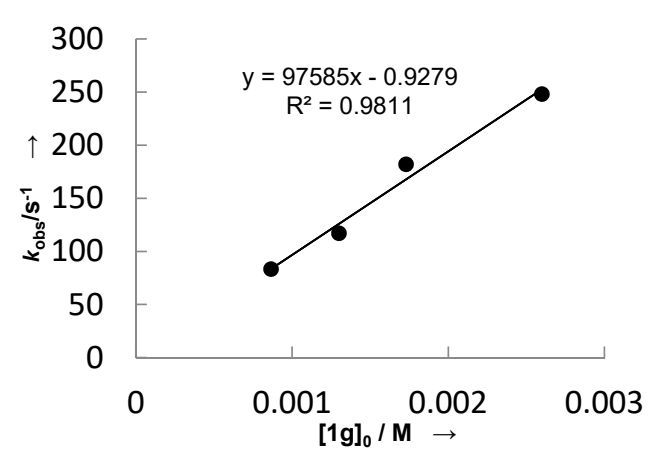

Table S31. Kinetics of the reactions of $\mathrm{CO}_{2}(\mathbf{1} \mathbf{h})$ with $\mathbf{2} \mathbf{j}$ in DMSO at $20{ }^{\circ} \mathrm{C}$ (deprotonated with $1.00-1.05$ equiv. $\mathrm{KO} t \mathrm{Bu}$, stopped-flow $\mathrm{UV}$-Vis spectrometer, $\lambda=265 \mathrm{~nm}$, Operator 1 ).

\begin{tabular}{ccc}
{$[\mathbf{2} \mathbf{j}] / \mathbf{M}$} & {$[\mathbf{1 h}] / \mathbf{M}$} & $\boldsymbol{k}_{\mathbf{o b s}} / \mathbf{s}^{\mathbf{- 1}}$ \\
$7.00 \times 10^{-5}$ & $6.83 \times 10^{-4}$ & $2.07 \times 10^{2}$ \\
$7.00 \times 10^{-5}$ & $1.02 \times 10^{-3}$ & $2.86 \times 10^{2}$ \\
$7.00 \times 10^{-5}$ & $1.35 \times 10^{-3}$ & $3.54 \times 10^{2}$ \\
$7.00 \times 10^{-5}$ & $1.68 \times 10^{-3}$ & $4.16 \times 10^{2}$ \\
\hline \multicolumn{2}{c}{$\left.\boldsymbol{k}_{\mathbf{2}}=\mathbf{( 2 . 0 9} \pm \mathbf{0 . 0 7}\right) \times \mathbf{1 0}^{\mathbf{5}} \mathbf{M}^{-\mathbf{1}} \mathbf{s}^{-\mathbf{1}}$}
\end{tabular}

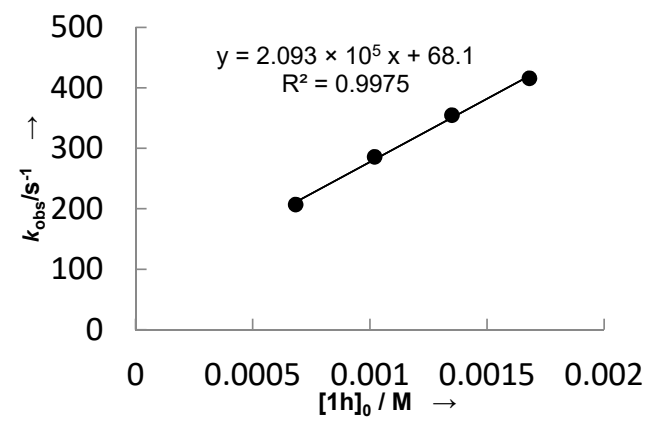

Table S32. Kinetics of the reactions of $\mathrm{CO}_{2}(\mathbf{1} \mathbf{h})$ with $\mathbf{2} \mathbf{j}$ in DMSO at $20{ }^{\circ} \mathrm{C}$ (deprotonated with $1.00-1.05$ equiv. $\mathrm{KO} t \mathrm{Bu}$ by using the double-mixing option of the stopped-flow $\mathrm{UV}$-Vis spectrometer, $\lambda=265 \mathrm{~nm}$, Operator 2).

\begin{tabular}{ccc}
{$[\mathbf{2} \mathbf{j}] / \mathbf{M}$} & {$[\mathbf{1} \mathbf{h}] / \mathbf{M}$} & $\boldsymbol{k}_{\mathbf{o b s}} / \mathbf{s}^{\mathbf{- 1}}$ \\
$7.00 \times 10^{-5}$ & $6.83 \times 10^{-4}$ & $1.43 \times 10^{2}$ \\
$7.00 \times 10^{-5}$ & $9.08 \times 10^{-4}$ & $1.90 \times 10^{2}$ \\
$7.00 \times 10^{-5}$ & $1.13 \times 10^{-3}$ & $2.38 \times 10^{2}$ \\
$7.00 \times 10^{-5}$ & $1.35 \times 10^{-3}$ & $2.75 \times 10^{2}$ \\
$7.00 \times 10^{-5}$ & $1.57 \times 10^{-3}$ & $3.07 \times 10^{2}$ \\
$7.00 \times 10^{-5}$ & $1.79 \times 10^{-3}$ & $3.52 \times 10^{2}$ \\
\hline \multicolumn{2}{c}{$\left.\boldsymbol{k}_{\mathbf{2}}=\mathbf{( 1 . 8 4} \pm \mathbf{0 . 0 6}\right) \times \mathbf{1 0}^{\mathbf{5}} \mathbf{M}^{-\mathbf{1}} \mathbf{s}^{-1}$}
\end{tabular}

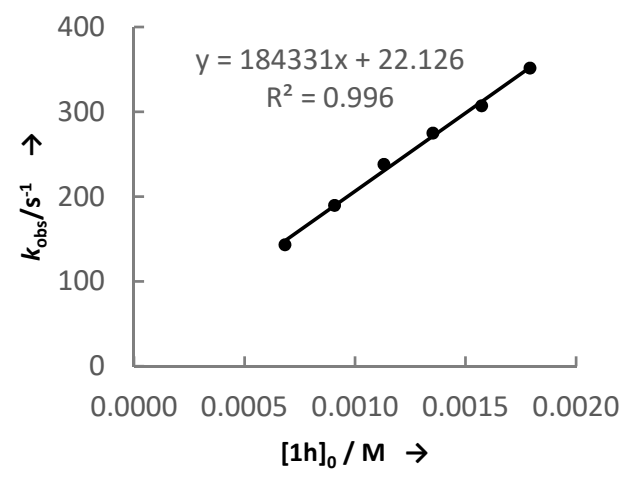




\section{NMR Kinetics of the Reactions of $1 b$ with 30 and $3 p$}

Scheme S1. The reaction rate law of bimolecular reactions

$$
\begin{gathered}
\mathrm{A}+\mathrm{B} \stackrel{k}{\longrightarrow} \mathrm{P} \quad \frac{\mathrm{d}[\mathrm{A}]}{\mathrm{d} t}=-k[\mathrm{~A}][\mathrm{B}] \\
\text { if }[\mathrm{A}]=[\mathrm{B}], \quad \frac{\mathrm{d}[\mathrm{A}]}{\mathrm{d} t}=-k[\mathrm{~A}]^{2} \quad \frac{1}{\underset{\text { integration }}{[} \frac{1}{[\mathrm{~A}]_{t}}-\frac{1}{[\mathrm{~A}]_{0}}=k t}
\end{gathered}
$$

As shown in Scheme S1, for identical concentrations of both starting materials, the correlation between $1 /[\mathrm{A}]_{\mathrm{t}}-1 /[\mathrm{A}]_{0}$ and reaction time $t$ is linear, and the slope equals the second-order rate constant $k_{2}$. Thus we determined second-order constants $k_{2}$ for reactions of $\mathbf{1 b}$ with 3o,p by plotting the correlation between $\left(1 /[3]_{\mathrm{t}}-1 /[]_{0}\right)$ and the corresponding reaction time $t$.

Determination of $k_{2}$ of reaction of $1 \mathrm{~b}$ with 30 in $\mathrm{CD}_{2} \mathrm{Cl}_{2}$ at $20^{\circ} \mathrm{C}$<smiles>Cc1ccc(S(=O)(=O)N([O-])O)cc1</smiles>

$1 \mathrm{~b}$<smiles>CCOC=CCC(=O)OCC</smiles>

30<smiles>CCOC1CC(=O)N1[123I]</smiles>

4 bo

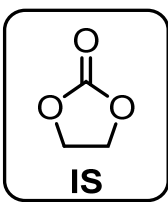

internal standard

To start the reaction, tosyl isocyanate $\mathbf{1 b}(94 \mathrm{mg}, 0.48 \mathrm{mmol}$ ), enol ether $\mathbf{3 o}$ (34 $\mathrm{mg}, 0.47 \mathrm{mmol}$ ), and ethylene carbonate IS (11 mg, $0.125 \mathrm{mmol}$, used as internal integration standard) were dissolved in $1.0 \mathrm{~mL} \mathrm{CD}_{2} \mathrm{Cl}_{2}$. The kinetics of the reaction was monitored by using time-resolved ${ }^{1} \mathrm{H}$ NMR spectroscopy. Concentrations of $\mathbf{3 o}$ were calculated from the ratio of the integral of the proton resonances of 30 centered at $\delta_{\mathrm{H}} 4.16 \mathrm{ppm}(\mathrm{dd})$ to the integral of the methylene resonances of the internal standard IS at $\delta_{\mathrm{H}} 4.48 \mathrm{ppm}(\mathrm{s})$.

As shown in Figure S2, we determined $k_{2}=1.6 \times 10^{-3} \mathrm{M}^{-1} \mathrm{~s}^{-1}$ for the reaction of $\mathbf{1 b}$ with $\mathbf{3 o}$ in $\mathrm{CD}_{2} \mathrm{Cl}_{2}$ at $20^{\circ} \mathrm{C}$. 


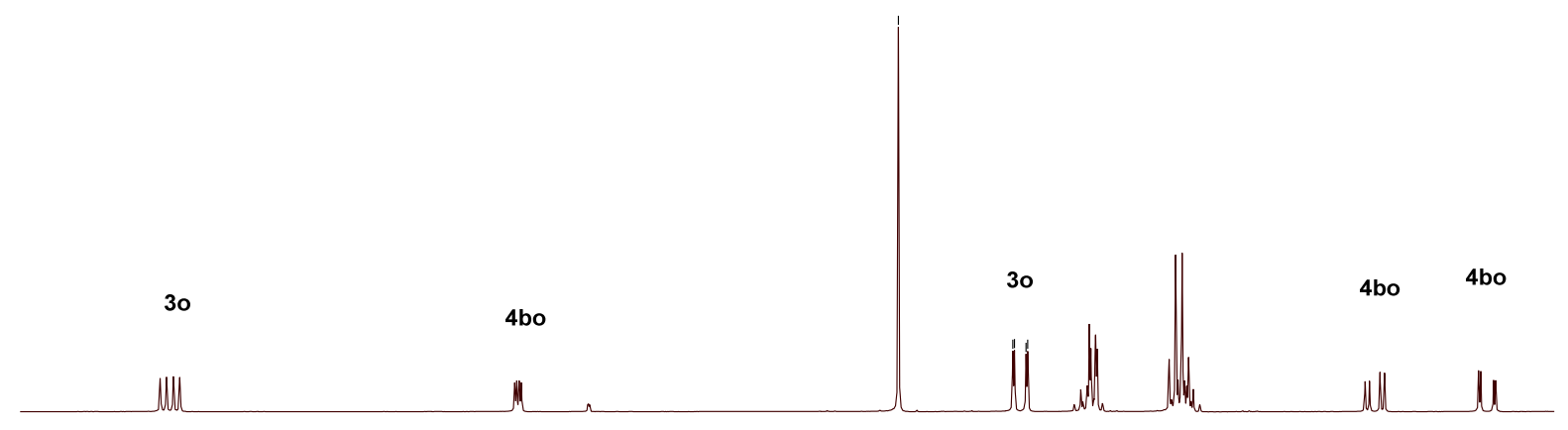

Figure S1. ${ }^{1} \mathrm{H}$ NMR spectrum $(400 \mathrm{MHz})$ of reaction of $\mathbf{1 b}$ and $\mathbf{3 o}$ in the presence of $\mathbf{I S}$ in $\mathrm{CD}_{2} \mathrm{Cl}_{2}$ at $20^{\circ} \mathrm{C}$ after $403 \mathrm{~s}$.

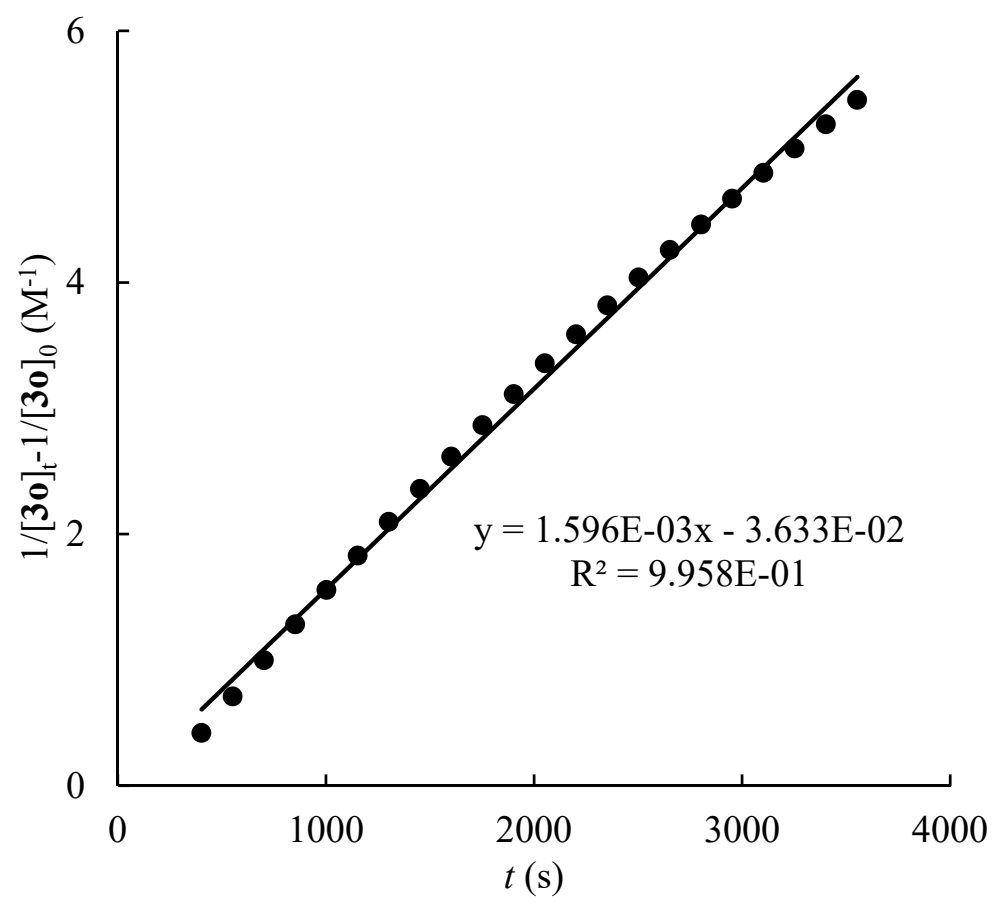

Figure S2. Correlation of $\left(1 /[\mathbf{3 o}]_{t}-1 /[\mathbf{3 o}]_{0}\right)$ during the reaction of $\mathbf{1 b}$ with $\mathbf{3 o}$ in $\mathrm{CD}_{2} \mathrm{Cl}_{2}$ at $20{ }^{\circ} \mathrm{C}$ vs the reaction time $t$. 
Determination of $k_{2}$ of reaction of $1 \mathrm{~b}$ with 30 in $\mathrm{CD}_{3} \mathrm{CN}$ at $20^{\circ} \mathrm{C}$

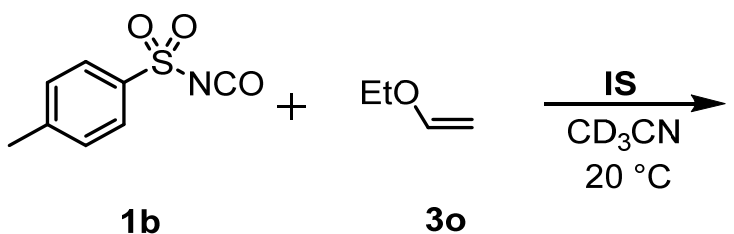<smiles>CCOC1CC(=O)N1[13O]</smiles>

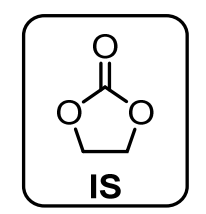

internal standard

To start the reaction, tosyl isocyanate $\mathbf{1 b}(71 \mathrm{mg}, 0.36 \mathrm{mmol})$, enol ether $\mathbf{3 o}(26 \mathrm{mg}, 0.36 \mathrm{mmol})$, and ethylene carbonate $\mathbf{I S}(7.5 \mathrm{mg}, 0.085 \mathrm{mmol})$ were dissolved in $1.0 \mathrm{~mL} \mathrm{CD}{ }_{3} \mathrm{CN}$. The kinetics of the reaction was monitored by using time-resolved ${ }^{1} \mathrm{H}$ NMR spectroscopy. Concentrations of 30 were calculated from the ratio of the integral of the proton resonances of $\mathbf{3 0}$ centered at $\delta_{\mathrm{H}} 4.16 \mathrm{ppm}(\mathrm{dd})$ to the integral of the methylene resonances of the internal standard IS at $\delta_{\mathrm{H}}$ $4.45 \mathrm{ppm}(\mathrm{s})$.

As shown in Figure S4, we determined $k_{2}=8.0 \times 10^{-3} \mathrm{M}^{-1} \mathrm{~s}^{-1}$ for the reaction of $\mathbf{1 b}$ with $\mathbf{3 o}$ in $\mathrm{CD}_{3} \mathrm{CN}$ at $20^{\circ} \mathrm{C}$.

$$
\begin{aligned}
& \text { 낭요오오 의 }
\end{aligned}
$$

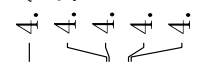

IS

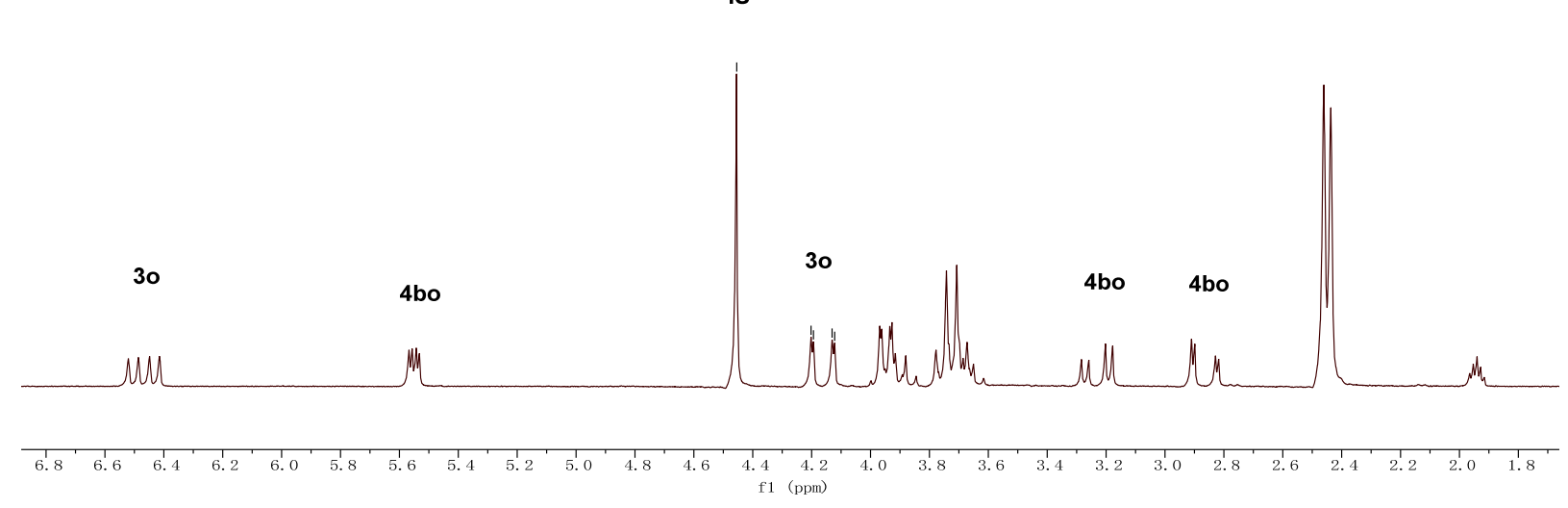

Figure S3. ${ }^{1} \mathrm{H}$ NMR spectrum $(200 \mathrm{MHz})$ of reaction of $\mathbf{1 b}$ and $\mathbf{3 o}$ in the presence of IS in $\mathrm{CD}_{3} \mathrm{CN}$ at $20{ }^{\circ} \mathrm{C}$ after $220 \mathrm{~s}$. 


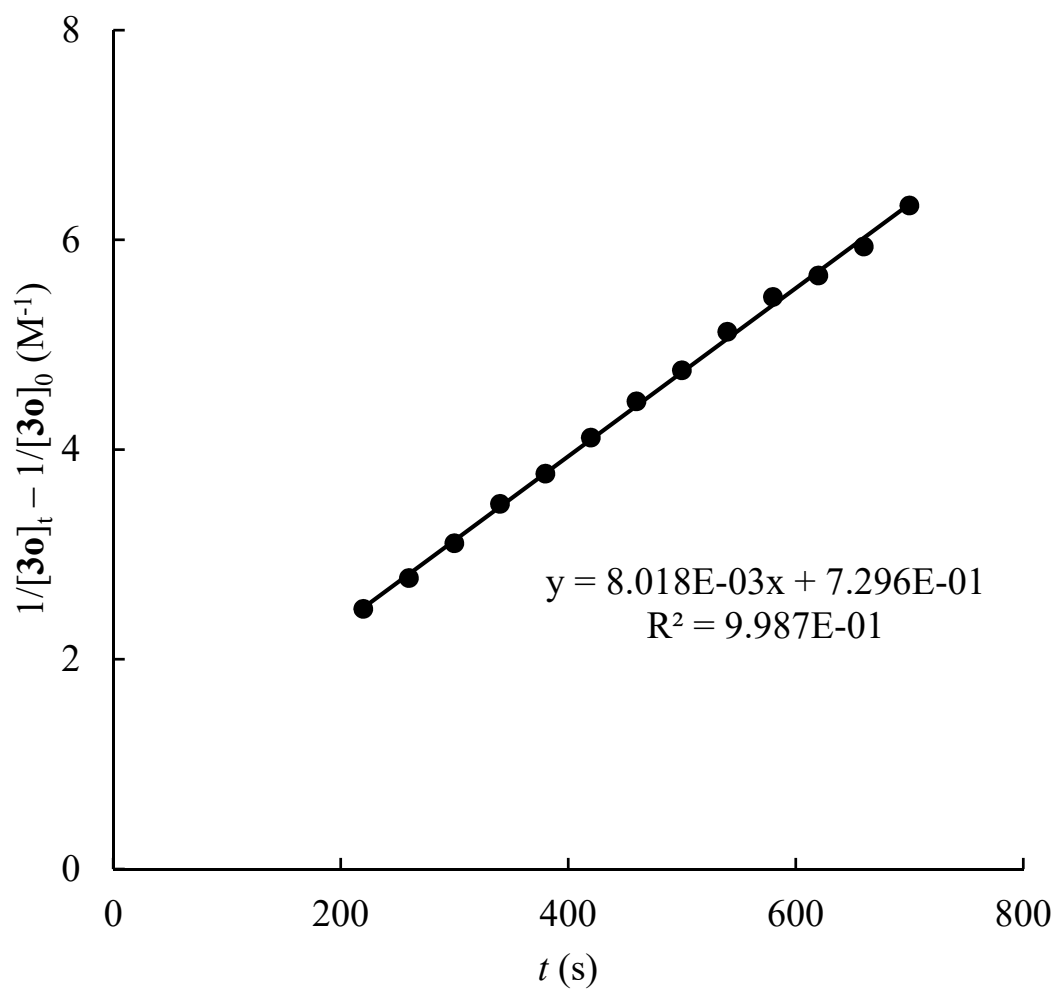

Figure S4. Correlation of $\left(1 /[\mathbf{3 o}]_{t}-1 /[\mathbf{3 o}]_{0}\right)$ during the reaction of $\mathbf{1 b}$ with $\mathbf{3 o}$ in $\mathrm{CD}_{3} \mathrm{CN}$ at $20{ }^{\circ} \mathrm{C}$ vs the reaction time $t$. 
Determination of $k_{2}$ of reaction of $1 \mathrm{~b}$ with $3 p$ in $\mathrm{CD}_{2} \mathrm{Cl}_{2}$ at $20^{\circ} \mathrm{C}$

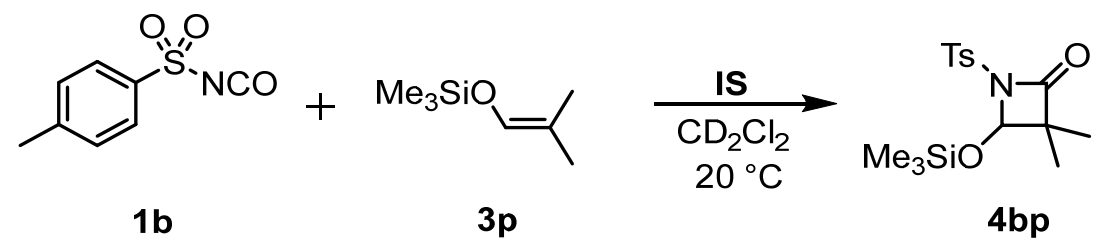

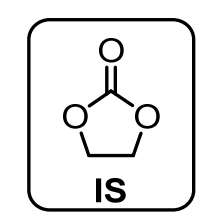

internal standard

To start the reaction, tosyl isocyanate $\mathbf{1 b}(81 \mathrm{mg}, 0.41 \mathrm{mmol})$, enol ether $\mathbf{3 p}$ ( $58 \mathrm{mg}, 0.40 \mathrm{mmol})$, and ethylene carbonate IS $(9.3 \mathrm{mg}, 0.11 \mathrm{mmol})$ were dissolved in $1.0 \mathrm{~mL} \mathrm{CD}_{2} \mathrm{Cl}_{2}$. The kinetics of the reaction was monitored by using time-resolved ${ }^{1} \mathrm{H}$ NMR spectroscopy. Concentrations of $3 \mathbf{p}$ were calculated from the ratio of the integral of the proton resonances of $\mathbf{3 p}$ centered at $\delta_{\mathrm{H}} 1.55 \mathrm{ppm}(\mathrm{dd})$ to the integral of the methylene resonances of the internal standard IS at $\delta_{\mathrm{H}}$ $4.49 \mathrm{ppm}(\mathrm{s})$.

As shown in Figure S6, we determined $k_{2}=1.5 \times 10^{-3} \mathrm{M}^{-1} \mathrm{~s}^{-1}$ for the reaction of $\mathbf{1 b}$ with $\mathbf{3 p}$ in $\mathrm{CD}_{2} \mathrm{Cl}_{2}$ at $20^{\circ} \mathrm{C}$.

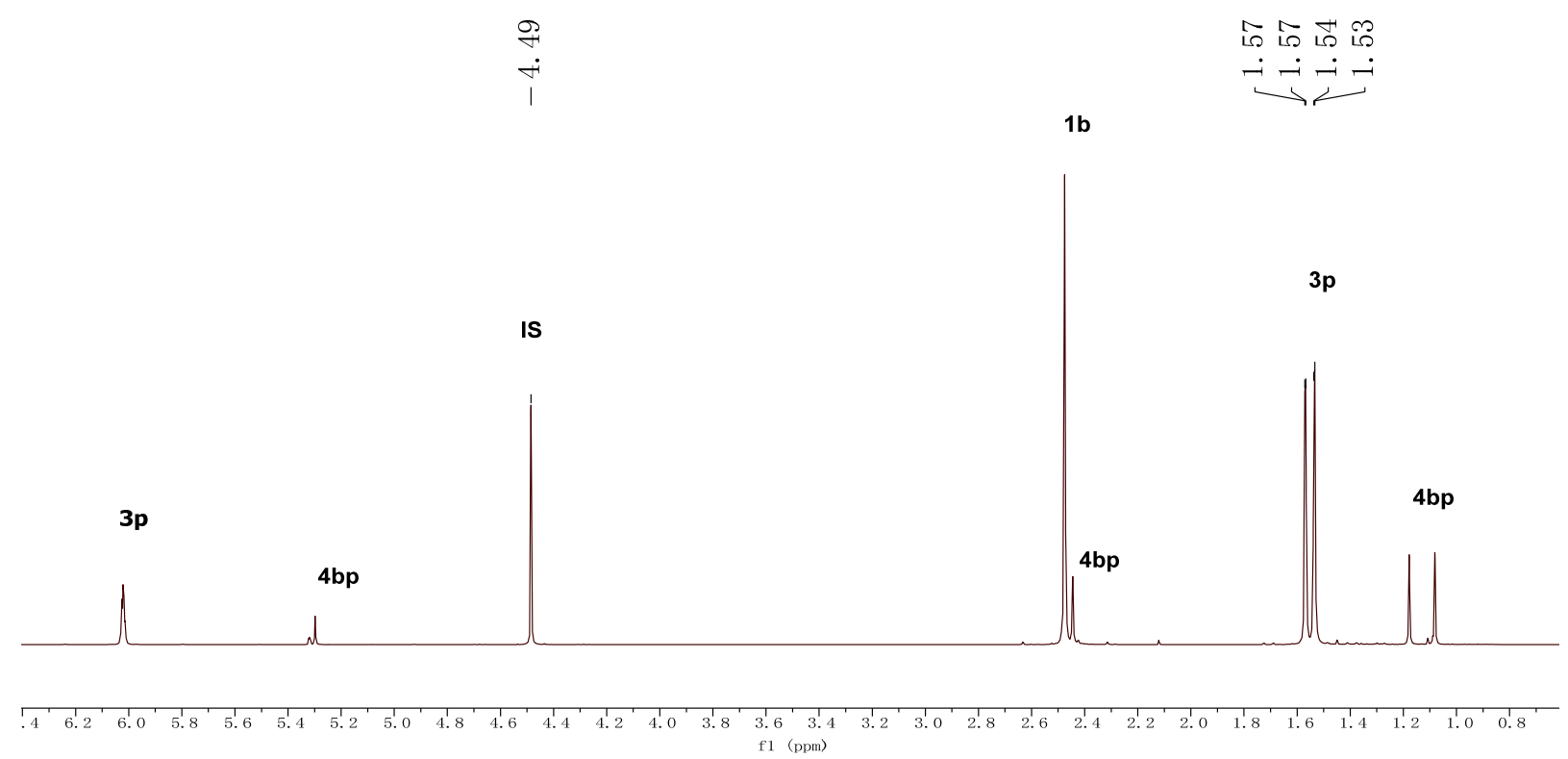

Figure S5. ${ }^{1} \mathrm{H}$ NMR spectrum (400 MHz) of reaction of $\mathbf{1 b}$ and $\mathbf{3 p}$ in the presence of IS in $\mathrm{CD}_{2} \mathrm{Cl}_{2}$ at $20^{\circ} \mathrm{C}$ after $364 \mathrm{~s}$. 


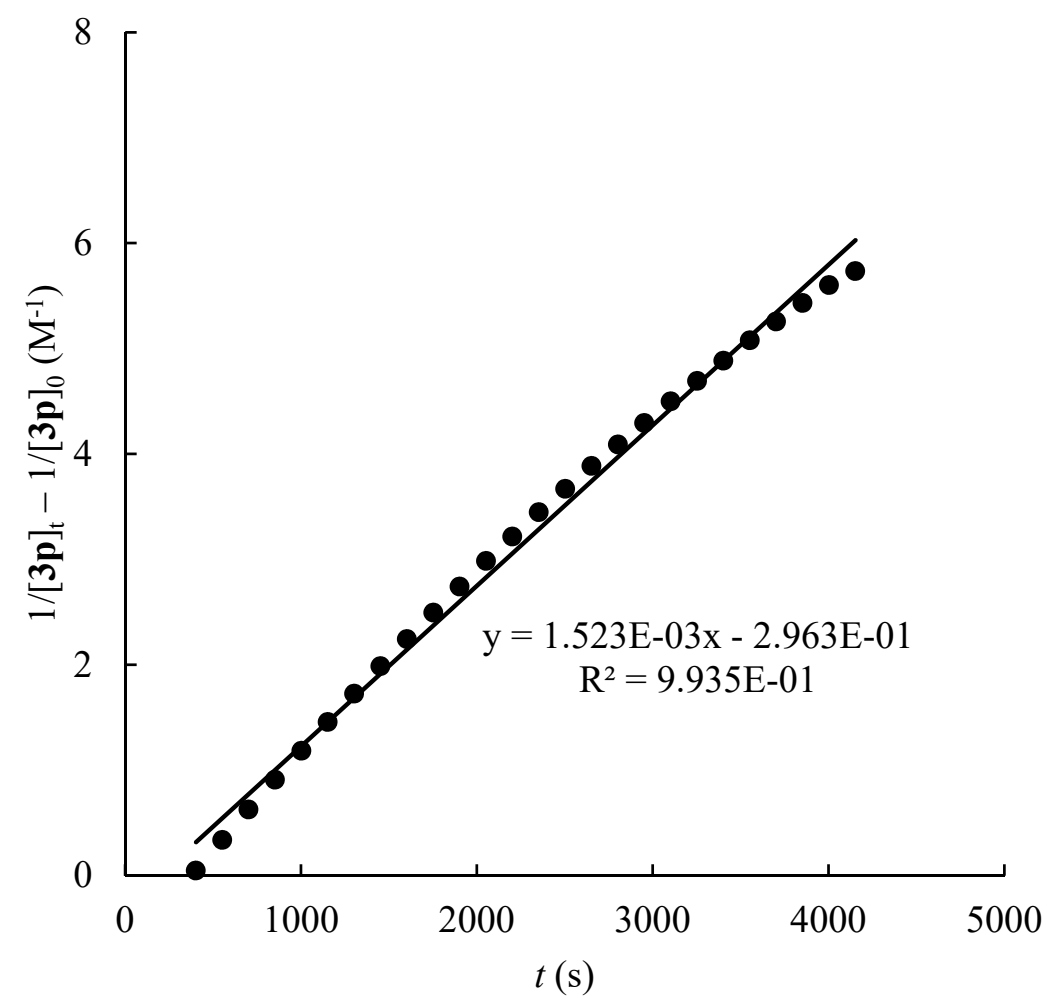

Figure S6. Correlation of $\left(1 /[\mathbf{3} \mathbf{p}]_{\mathrm{t}}-1 /[\mathbf{3} \mathbf{p}]_{0}\right)$ during the reaction of $\mathbf{1 b}$ with $\mathbf{3 p}$ in $\mathrm{CD}_{2} \mathrm{Cl}_{2}$ at $20^{\circ} \mathrm{C}$ vs the reaction time $t$. 
Determination of $k_{2}$ of reaction of $1 \mathrm{~b}$ with $3 p$ in $\mathrm{CD}_{3} \mathrm{CN}$ at $20^{\circ} \mathrm{C}$<smiles>Cc1ccc(S(=O)(=O)N=O)cc1</smiles>

$1 b$

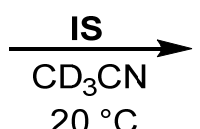

$3 p$

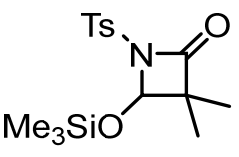

$4 \mathrm{bp}$

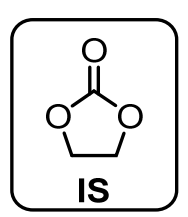

internal standard

To start the reaction, tosyl isocyanate $\mathbf{1 b}(88 \mathrm{mg}, 0.45 \mathrm{mmol})$, enol ether $\mathbf{3 p}$ ( $65 \mathrm{mg}, 0.45 \mathrm{mmol}$ ), and ethylene carbonate $\mathbf{I S}\left(9.7 \mathrm{mg}(0.11 \mathrm{mmol})\right.$ were dissolved in $1.0 \mathrm{~mL} \mathrm{CD}{ }_{3} \mathrm{CN}$. The kinetics of the reaction was monitored by using time-resolved ${ }^{1} \mathrm{H}$ NMR spectroscopy. Concentrations of $\mathbf{3 p}$ were calculated from the ratio of the integral of the proton resonances of $\mathbf{3 p}$ centered at $\delta_{\mathrm{H}} 1.53 \mathrm{ppm}(\mathrm{dd})$ to the integral of the methylene resonances of the internal standard IS at $\delta_{\mathrm{H}}$ $4.46 \mathrm{ppm}(\mathrm{s})$.

We determined $k_{2}=7.0 \times 10^{-3} \mathrm{M}^{-1} \mathrm{~s}^{-1}$ for the reaction of $\mathbf{1 b}$ with $\mathbf{3 p}$ in $\mathrm{CD}_{3} \mathrm{CN}$ at $20{ }^{\circ} \mathrm{C}$ (Figure S8).
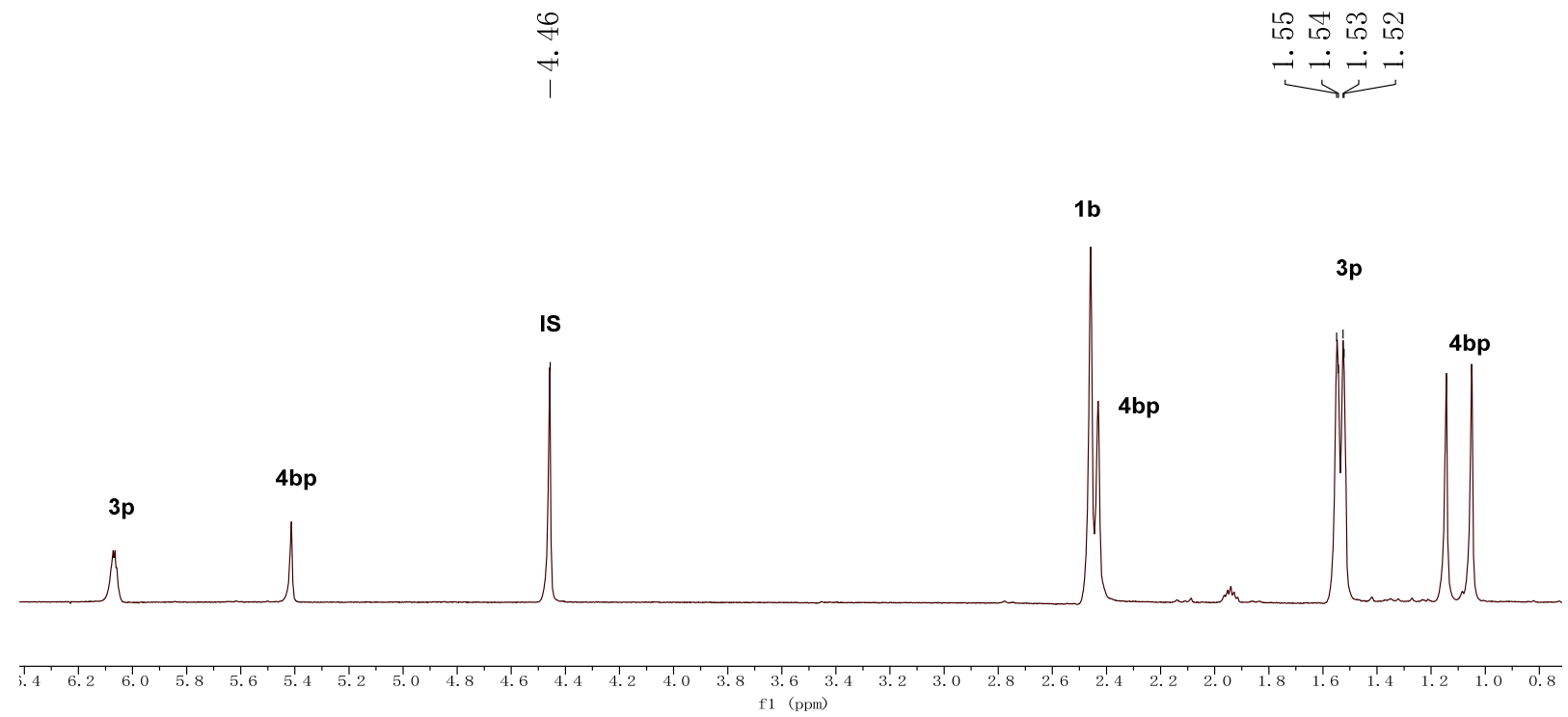

Figure S7. ${ }^{1} \mathrm{H}$ NMR spectrum $(200 \mathrm{MHz})$ of reaction of $\mathbf{1 b}$ and $\mathbf{3 p}$ in the presence of IS in $\mathrm{CD}_{3} \mathrm{CN}$ at $20{ }^{\circ} \mathrm{C}$ after $205 \mathrm{~s}$. 


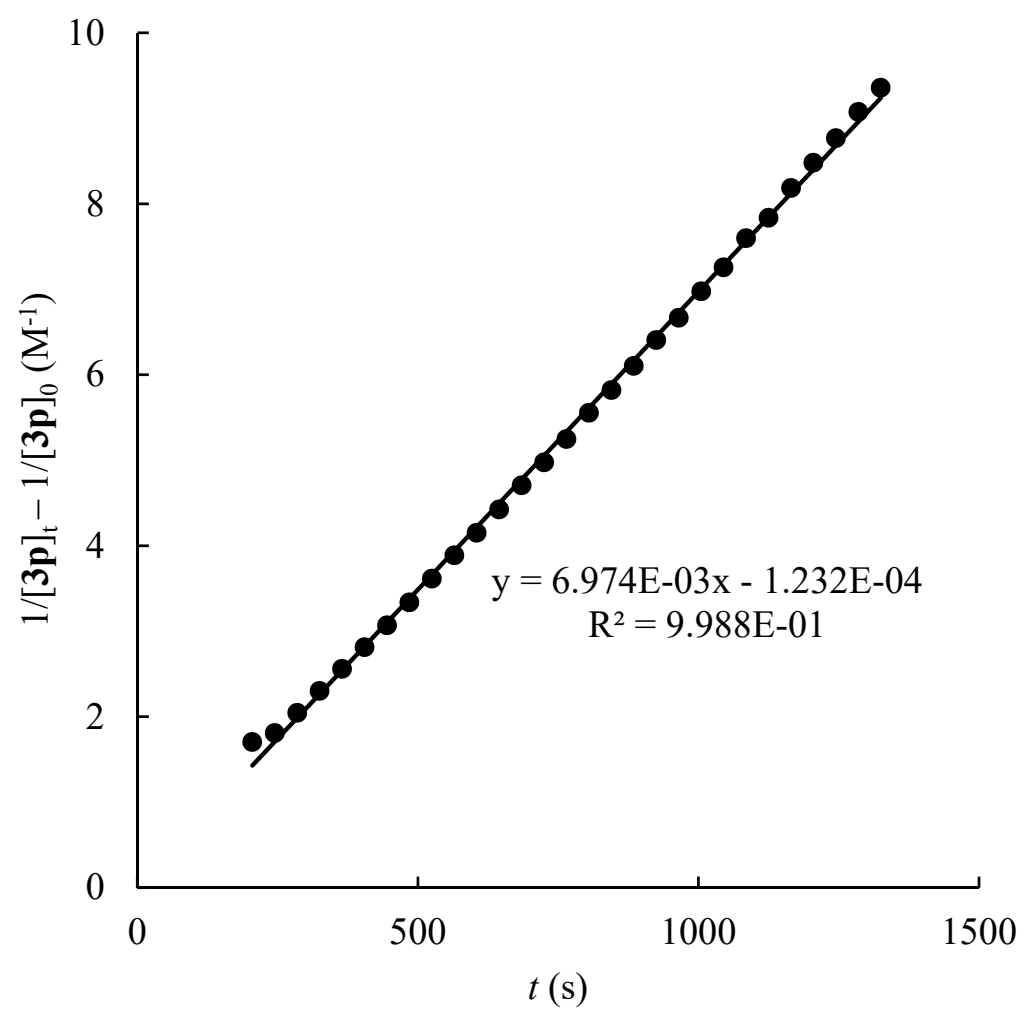

Figure S8. Correlation of $\left(1 /[\mathbf{3} \mathbf{p}]_{\mathrm{t}}-1 /[\mathbf{3} \mathbf{p}]_{0}\right)$ during the reaction of $\mathbf{1 b}$ with $\mathbf{3 p}$ in $\mathrm{CD}_{3} \mathrm{CN}$ at $20{ }^{\circ} \mathrm{C}$ vs the reaction time $t$. 


\section{Nucleophilic Reactivity of Carbanions 2 in $\mathrm{MeCN}$}

The rates of all investigated reactions were determined spectrophotometrically by following the disappearance of the UV/Vis absorptions of the electrophiles 10. The temperature of the solutions during all kinetics studies was kept constant $\left(20.0 \pm 0.1^{\circ} \mathrm{C}\right)$ by using a circulating bath thermostat. The kinetics investigations were performed with a high excess of the carbanions 2 over the electrophiles to achieve first-order kinetics. The kinetics were followed by using a commercial stopped-flow spectrophotometer system. Rate constants $k_{\text {obs }}\left(\mathrm{s}^{-1}\right)$ were obtained by fitting the single exponential decay function $A_{\mathrm{t}}=A_{0} \exp \left(-k_{\mathrm{obs}} t\right)+C$ (exponential decrease) to the observed time-dependent absorbance (at least 5 kinetic runs for each nucleophile concentration were averaged). Second-order rate constants $k_{2}\left(\mathrm{M}^{-1} \mathrm{~s}^{-1}\right)$ were derived from the slopes of the linear correlations of $k_{\mathrm{obs}}$ with electrophile concentrations.

Table S33. Reference electrophiles ${ }^{\mathrm{S}} 16$ for deriving the nucleophile-specific reactivity parameters $N$ and $s \mathrm{~N}$ for the carbanions $\mathbf{2}$ in acetonitrile.

(in MeCN)


Table S34. Kinetics of the reactions of $\mathbf{2 b}$ with $\mathbf{1 0 b}$ in $\mathrm{CH}_{3} \mathrm{CN}$ at $20^{\circ} \mathrm{C}$ (deprotonated with $1.00-1.05$ equiv. $\mathrm{KO} t \mathrm{Bu}$, conventional $\mathrm{UV}$-Vis spectrometer, $\lambda=631 \mathrm{~nm}$ ).

\begin{tabular}{ccc}
{$[\mathbf{1 0 b}] / \mathbf{M}$} & {$[\mathbf{2 b}] / \mathbf{M}$} & $\boldsymbol{k}_{\mathbf{o b s}} / \mathbf{s}^{-1}$ \\
$2.50 \times 10^{-5}$ & $2.49 \times 10^{-4}$ & $3.06 \times 10^{2}$ \\
$2.50 \times 10^{-5}$ & $3.74 \times 10^{-4}$ & $4.93 \times 10^{2}$ \\
$2.50 \times 10^{-5}$ & $4.99 \times 10^{-4}$ & $6.80 \times 10^{2}$ \\
$2.50 \times 10^{-5}$ & $6.23 \times 10^{-4}$ & $8.80 \times 10^{2}$ \\
$2.50 \times 10^{-5}$ & $7.48 \times 10^{-4}$ & $1.05 \times 10^{3}$ \\
\hline \multicolumn{2}{c}{$k_{2}=(1.50 \pm 0.02) \times 10^{6} \mathrm{M}^{-1} \mathrm{~s}^{-1}$}
\end{tabular}

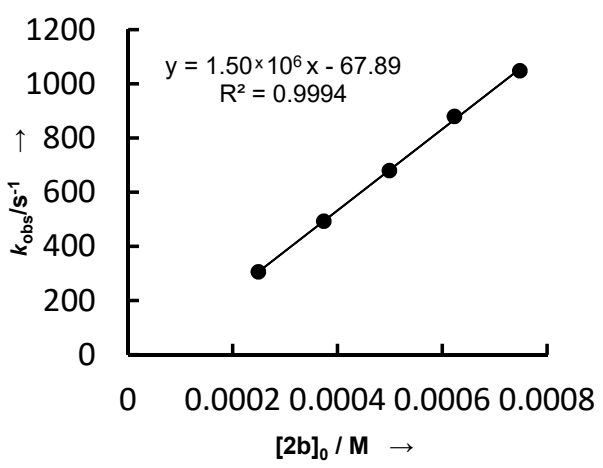

Table S35. Kinetics of the reactions of $\mathbf{2 b}$ with $\mathbf{1 0 d}$ in $\mathrm{CH}_{3} \mathrm{CN}$ at $20^{\circ} \mathrm{C}$ (deprotonated with $1.00-1.05$ equiv. $\mathrm{KO} t \mathrm{Bu}$, conventional $\mathrm{UV}$-Vis spectrometer, $\lambda=420 \mathrm{~nm}$ ).

\begin{tabular}{ccc}
{$[\mathbf{1 0 d}] / \mathbf{M}$} & {$[\mathbf{2 b}] / \mathbf{M}$} & $\boldsymbol{k}_{\mathbf{o b s}} / \mathbf{s}^{-1}$ \\
$2.50 \times 10^{-5}$ & $5.01 \times 10^{-4}$ & 2.26 \\
$2.50 \times 10^{-5}$ & $7.52 \times 10^{-4}$ & 3.44 \\
$2.50 \times 10^{-5}$ & $1.00 \times 10^{-3}$ & 4.61 \\
$2.50 \times 10^{-5}$ & $1.25 \times 10^{-3}$ & 5.70 \\
$2.50 \times 10^{-5}$ & $1.50 \times 10^{-3}$ & 6.88 \\
\hline \multicolumn{3}{c}{$k_{2}=(4.61 \pm 0.04) \times 10^{3} \mathrm{M}^{-1} \mathrm{~s}^{-1}$}
\end{tabular}

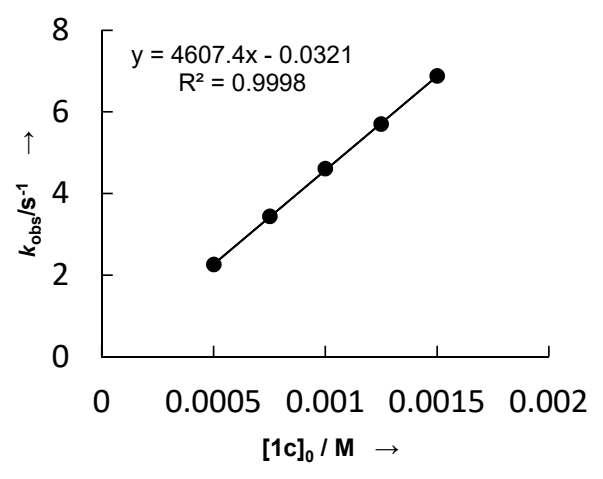

Table S36. Kinetics of the reactions of $\mathbf{2 b}$ with $\mathbf{1 0 e}$ in $\mathrm{CH}_{3} \mathrm{CN}$ at $20{ }^{\circ} \mathrm{C}$ (deprotonated with $1.00-1.05$ equiv. $\mathrm{KO} t \mathrm{Bu}$, conventional $\mathrm{UV}-\mathrm{V}$ is spectrometer, $\lambda=533 \mathrm{~nm}$ ).

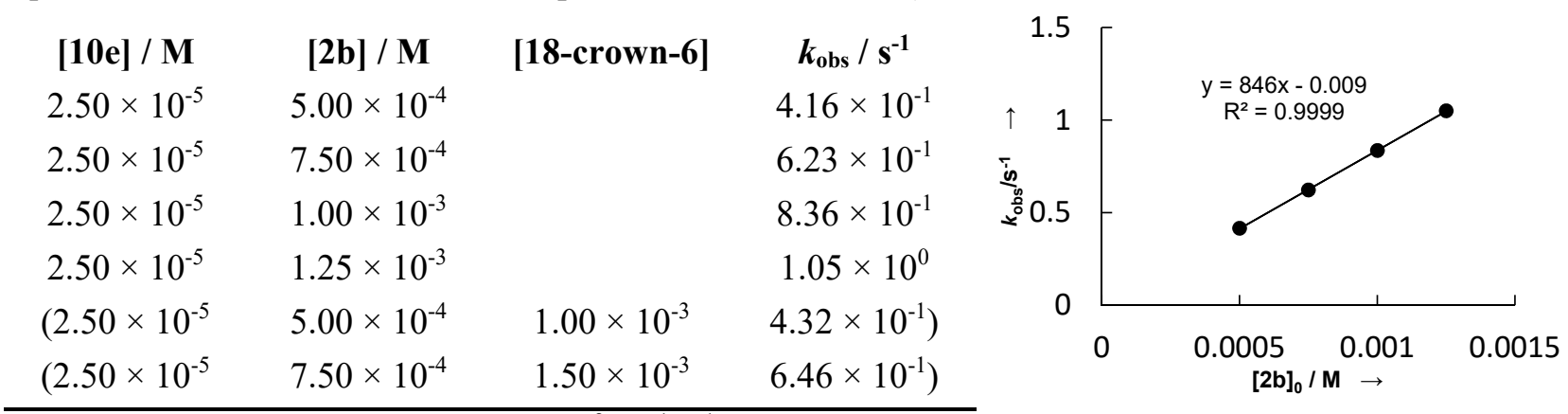




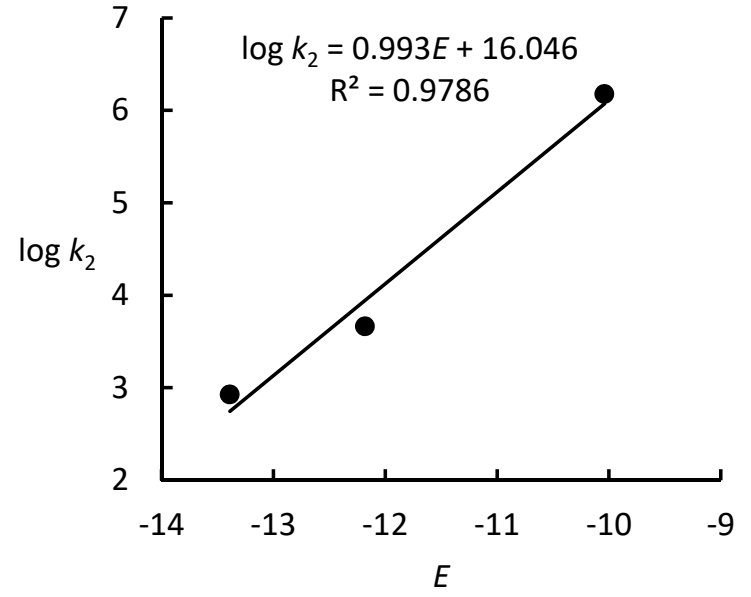

Figure S9. Correlation of $\log k_{2}$ for the reactions of carbanion $\mathbf{2 b}$ with reference electrophiles 10 vs the electrophilicity parameters $E$ of $\mathbf{1 0}$.

For 2b (in $\mathrm{MeCN}): N=16.15, s_{\mathrm{N}}=0.99$

Table S37. Kinetics of the reactions of $2 \mathbf{c}$ with $\mathbf{1 0 a}$ in $\mathrm{CH}_{3} \mathrm{CN}$ at $20^{\circ} \mathrm{C}$ (deprotonated with $1.00-1.05$ equiv. $\mathrm{KO} t \mathrm{Bu}$, conventional $\mathrm{UV}-\mathrm{Vis}$ spectrometer, $\lambda=635 \mathrm{~nm}$ ).

\begin{tabular}{ccc}
{$[\mathbf{1 0 a}] / \mathbf{M}$} & {$[\mathbf{2} \mathbf{c}] / \mathbf{M}$} & $\boldsymbol{k}_{\mathbf{o b s}} / \mathbf{s}^{-1}$ \\
$1.30 \times 10^{-5}$ & $1.98 \times 10^{-4}$ & $1.55 \times 10^{2}$ \\
$1.30 \times 10^{-5}$ & $2.63 \times 10^{-4}$ & $2.35 \times 10^{2}$ \\
$1.30 \times 10^{-5}$ & $3.29 \times 10^{-4}$ & $3.17 \times 10^{2}$ \\
$1.30 \times 10^{-5}$ & $3.95 \times 10^{-4}$ & $4.30 \times 10^{2}$ \\
\hline \multicolumn{2}{c}{$k_{2}=(1.38 \pm 0.08) \times 10^{6} \mathrm{M}^{-1} \mathrm{~s}^{-1}$}
\end{tabular}

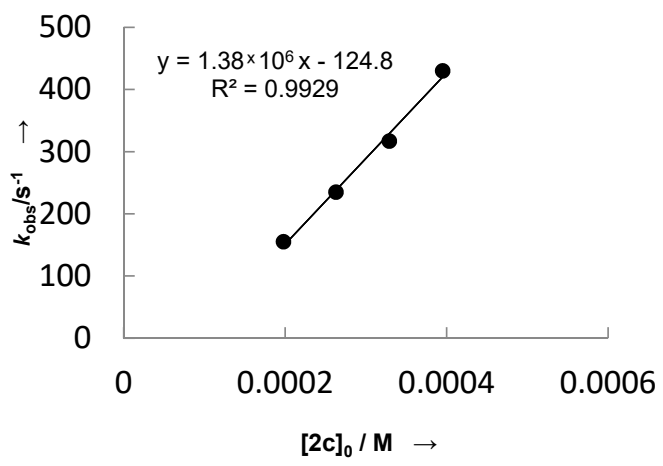

Table S38. Kinetics of the reactions of $\mathbf{2} \mathbf{c}$ with $\mathbf{1 0 b}$ in $\mathrm{CH}_{3} \mathrm{CN}$ at $20{ }^{\circ} \mathrm{C}$ (deprotonated with $1.00-1.05$ equiv. $\mathrm{KO} t \mathrm{Bu}$, conventional $\mathrm{UV}-\mathrm{V}$ is spectrometer, $\lambda=631 \mathrm{~nm}$ ).

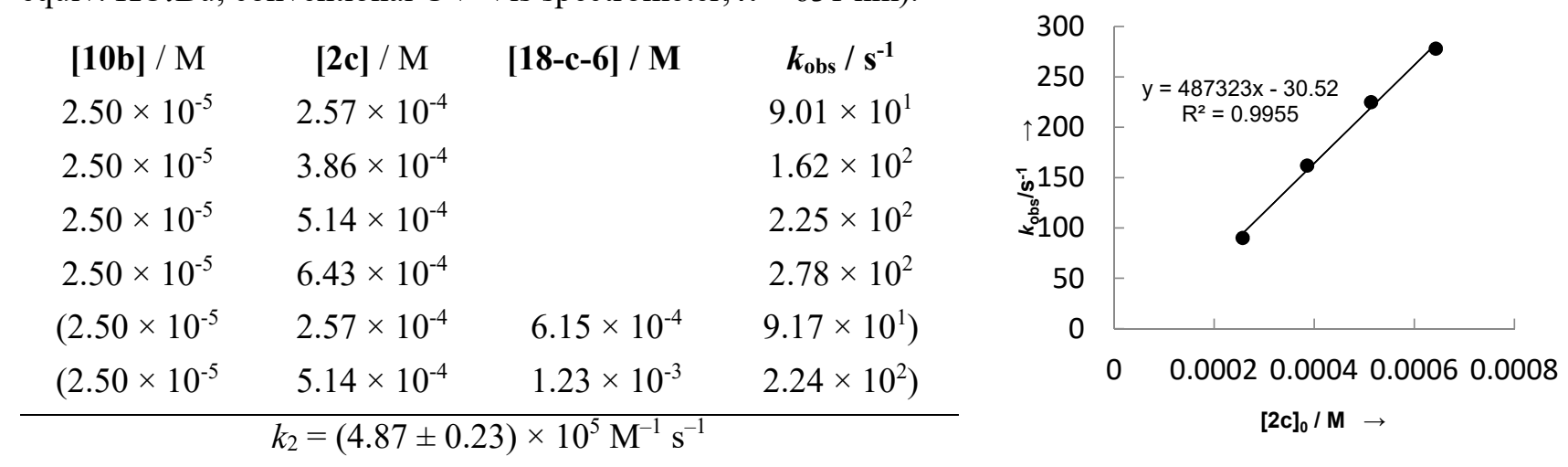


Table S39. Kinetics of the reactions of $2 \mathbf{c}$ with $\mathbf{1 0} \mathbf{c}$ in $\mathrm{CH}_{3} \mathrm{CN}$ at $20^{\circ} \mathrm{C}$ (deprotonated with $1.00-1.05$ equiv. $\mathrm{KO} t \mathrm{Bu}$, conventional $\mathrm{UV}-\mathrm{Vis}$ spectrometer, $\lambda=380 \mathrm{~nm}$ ).

\begin{tabular}{ccc}
{$[\mathbf{1 0 c}] / \mathbf{M}$} & {$[\mathbf{2 c}] / \mathbf{M}$} & $\boldsymbol{k}_{\text {obs }} / \mathbf{s}^{-1}$ \\
$2.50 \times 10^{-5}$ & $2.51 \times 10^{-4}$ & $6.91 \times 10^{-1}$ \\
$2.50 \times 10^{-5}$ & $3.76 \times 10^{-4}$ & $9.63 \times 10^{-1}$ \\
$2.50 \times 10^{-5}$ & $5.01 \times 10^{-4}$ & 1.37 \\
$2.50 \times 10^{-5}$ & $6.27 \times 10^{-4}$ & 1.91 \\
$2.50 \times 10^{-5}$ & $7.52 \times 10^{-4}$ & 2.32 \\
\hline \multicolumn{2}{c}{$k_{2}=(3.36 \pm 0.21) \times 10^{3} \mathrm{M}^{-1} \mathrm{~s}^{-1}$}
\end{tabular}

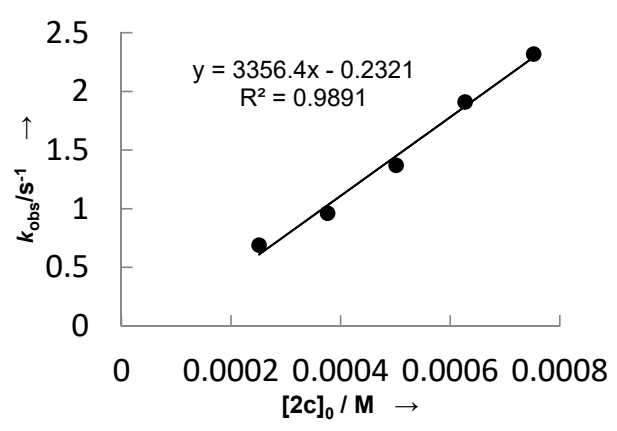

Table S40. Kinetics of the reactions of $\mathbf{2} \mathbf{c}$ with $\mathbf{1 0 d}$ in $\mathrm{CH}_{3} \mathrm{CN}$ at $20{ }^{\circ} \mathrm{C}$ (deprotonated with $1.00-1.05$ equiv. $\mathrm{KO} t \mathrm{Bu}$, conventional $\mathrm{UV}$-Vis spectrometer, $\lambda=420 \mathrm{~nm}$ ).

\begin{tabular}{ccc}
{$[\mathbf{1 0 d}] / \mathbf{M}$} & {$[\mathbf{2 c}] / \mathbf{M}$} & $\boldsymbol{k}_{\text {obs }} / \mathbf{s}^{-1}$ \\
$2.50 \times 10^{-5}$ & $2.51 \times 10^{-4}$ & $4.09 \times 10^{-1}$ \\
$2.50 \times 10^{-5}$ & $3.76 \times 10^{-4}$ & $5.83 \times 10^{-1}$ \\
$2.50 \times 10^{-5}$ & $5.01 \times 10^{-4}$ & $7.98 \times 10^{-1}$ \\
$2.50 \times 10^{-5}$ & $6.27 \times 10^{-4}$ & $9.93 \times 10^{-1}$ \\
$2.50 \times 10^{-5}$ & $7.52 \times 10^{-4}$ & 1.15 \\
\hline \multicolumn{3}{c}{$k_{2}=(1.51 \pm 0.05) \times 10^{3} \mathrm{M}^{-1} \mathrm{~s}^{-1}$}
\end{tabular}

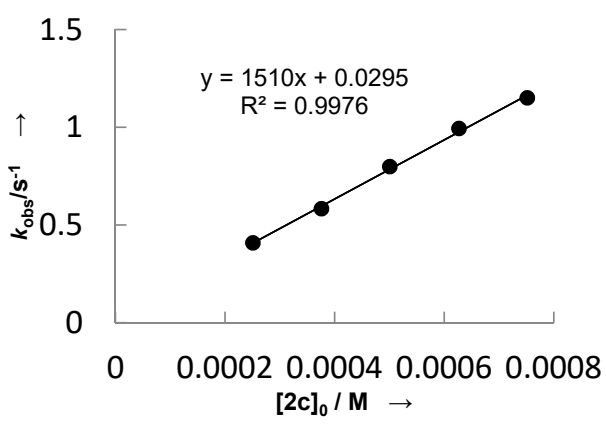

Table S41. Kinetics of the reactions of $2 \mathbf{c}$ with $\mathbf{1 0 e}$ in $\mathrm{CH}_{3} \mathrm{CN}$ at $20^{\circ} \mathrm{C}$ (deprotonated with $1.00-1.05$ equiv. $\mathrm{KO} t \mathrm{Bu}$, conventional $\mathrm{UV}-\mathrm{Vis}$ spectrometer, $\lambda=533 \mathrm{~nm}$ ).

\begin{tabular}{ccc}
{$[\mathbf{1 0 e}] / \mathbf{M}$} & {$[\mathbf{2 c}] / \mathbf{M}$} & $\boldsymbol{k}_{\text {obs }} / \mathbf{s}^{-1}$ \\
$2.50 \times 10^{-5}$ & $4.92 \times 10^{-4}$ & $1.25 \times 10^{-1}$ \\
$2.50 \times 10^{-5}$ & $6.14 \times 10^{-4}$ & $1.70 \times 10^{-1}$ \\
$2.50 \times 10^{-5}$ & $7.37 \times 10^{-4}$ & $1.97 \times 10^{-1}$ \\
$2.50 \times 10^{-5}$ & $8.60 \times 10^{-4}$ & $2.37 \times 10^{-1}$ \\
\hline \multicolumn{2}{c}{$k_{2}=(2.96 \pm 0.20) \times 10^{2} \mathrm{M}^{-1} \mathrm{~s}^{-1}$}
\end{tabular}
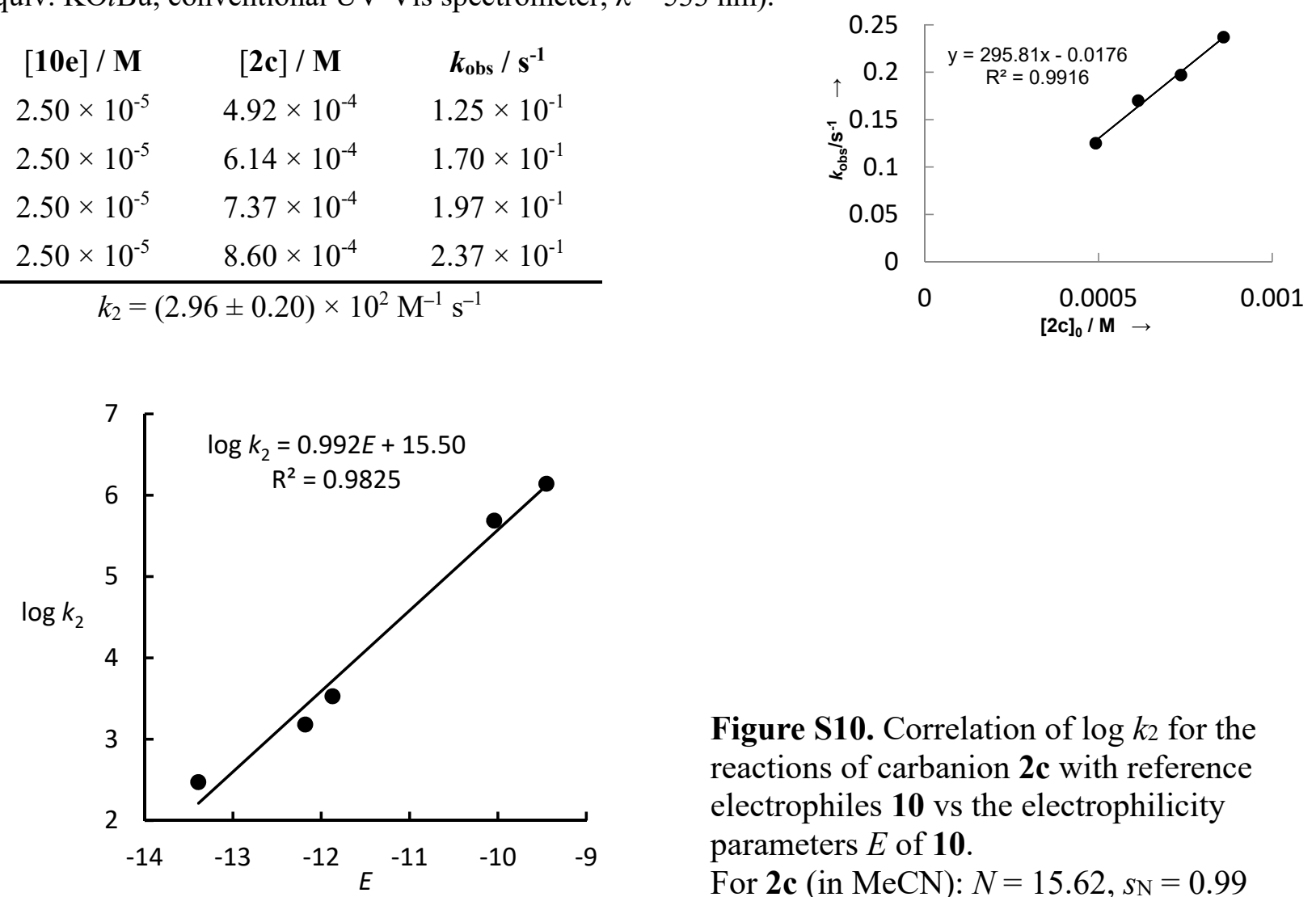

Figure S10. Correlation of $\log k_{2}$ for the reactions of carbanion $\mathbf{2 c}$ with reference electrophiles 10 vs the electrophilicity parameters $E$ of $\mathbf{1 0 .}$

For 2 c (in $\mathrm{MeCN}): N=15.62, s_{\mathrm{N}}=0.99$ 
Table S42. Kinetics of the reactions of $\mathbf{2 i}$ with $\mathbf{1 0 d}$ in $\mathrm{CH}_{3} \mathrm{CN}$ at $20{ }^{\circ} \mathrm{C}$ (deprotonated with $1.00-1.05$ equiv. $\mathrm{KO} t \mathrm{Bu}$, conventional $\mathrm{UV}-\mathrm{Vis}$ spectrometer, $\lambda=420 \mathrm{~nm}$ ).

\begin{tabular}{cccc}
{$[\mathbf{1 0 d}] / \mathrm{M}$} & {$[\mathbf{2 i}] / \mathrm{M}$} & {$[18-\mathrm{c}-6] / \mathrm{M}$} & $k_{\mathrm{obs}} / \mathrm{s}^{-1}$ \\
$2.50 \times 10^{-5}$ & $4.99 \times 10^{-4}$ & & $1.41 \times 10^{2}$ \\
$2.50 \times 10^{-5}$ & $7.48 \times 10^{-4}$ & & $2.21 \times 10^{2}$ \\
$2.50 \times 10^{-5}$ & $9.98 \times 10^{-4}$ & & $3.10 \times 10^{2}$ \\
$2.50 \times 10^{-5}$ & $1.25 \times 10^{-3}$ & & $4.07 \times 10^{2}$ \\
$\left(2.50 \times 10^{-5}\right.$ & $4.99 \times 10^{-4}$ & $1.00 \times 10^{-3}$ & $\left.1.29 \times 10^{2}\right)$ \\
$\left(2.50 \times 10^{-5}\right.$ & $7.48 \times 10^{-4}$ & $1.50 \times 10^{-3}$ & $\left.2.17 \times 10^{2}\right)$ \\
\hline \multicolumn{3}{c}{$k_{2}=(3.54 \pm 0.10) \times 10^{5} \mathrm{M}^{-1} \mathrm{~s}^{-1}$}
\end{tabular}

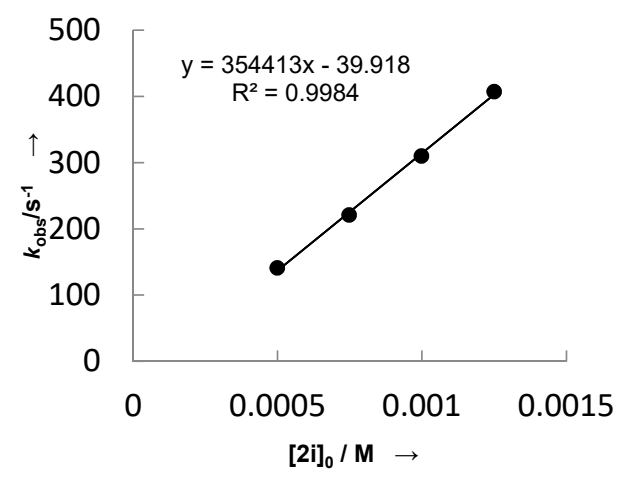

Table S43. Kinetics of the reactions of $\mathbf{2} \mathbf{i}$ with $\mathbf{1 0 f}$ in $\mathrm{CH}_{3} \mathrm{CN}$ at $20{ }^{\circ} \mathrm{C}$ (deprotonated with $1.00-1.05$ equiv. $\mathrm{KO} t \mathrm{Bu}$, conventional $\mathrm{UV}-\mathrm{Vis}$ spectrometer, $\lambda=374 \mathrm{~nm}$ ).

\begin{tabular}{ccc}
{$[\mathbf{1 0 f}] / \mathbf{M}$} & {$[\mathbf{2 i}] / \mathbf{M}$} & $\boldsymbol{k}_{\mathbf{o b s}} / \mathbf{s}^{-1}$ \\
$2.50 \times 10^{-5}$ & $4.99 \times 10^{-4}$ & 6.28 \\
$2.50 \times 10^{-5}$ & $7.48 \times 10^{-4}$ & $1.01 \times 10^{1}$ \\
$2.50 \times 10^{-5}$ & $9.98 \times 10^{-4}$ & $1.40 \times 10^{1}$ \\
$2.50 \times 10^{-5}$ & $1.25 \times 10^{-3}$ & $1.73 \times 10^{1}$ \\
$2.50 \times 10^{-5}$ & $1.50 \times 10^{-3}$ & $2.05 \times 10^{1}$ \\
\hline \multicolumn{2}{c}{$k_{2}=(1.42 \pm 0.04) \times 10^{4} \mathrm{M}^{-1} \mathrm{~s}^{-1}$}
\end{tabular}

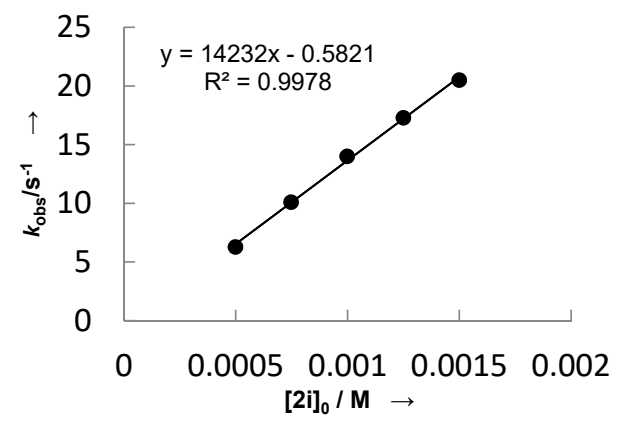

Table S44. Kinetics of the reactions of $\mathbf{2} \mathbf{i}$ with $\mathbf{1 0 g}$ in $\mathrm{CH}_{3} \mathrm{CN}$ at $20{ }^{\circ} \mathrm{C}$ (deprotonated with $1.00-1.05$ equiv. $\mathrm{KO} t \mathrm{Bu}$, conventional $\mathrm{UV}-\mathrm{V}$ is spectrometer, $\lambda=380 \mathrm{~nm}$ ).

\begin{tabular}{ccc}
{$[\mathbf{1 0 g}] / \mathbf{M}$} & {$[\mathbf{2 i}] / \mathbf{M}$} & $\boldsymbol{k}_{\mathbf{o b s}} / \mathbf{s}^{-1}$ \\
$2.50 \times 10^{-5}$ & $5.09 \times 10^{-4}$ & $4.49 \times 10^{-1}$ \\
$2.50 \times 10^{-5}$ & $7.63 \times 10^{-4}$ & $6.94 \times 10^{-1}$ \\
$2.50 \times 10^{-5}$ & $1.02 \times 10^{-3}$ & $9.12 \times 10^{-1}$ \\
$2.50 \times 10^{-5}$ & $1.27 \times 10^{-3}$ & 1.14 \\
$2.50 \times 10^{-5}$ & $1.53 \times 10^{-3}$ & 1.37 \\
\hline \multicolumn{2}{c}{$k_{2}=(8.98 \pm 0.09) \times 10^{2} \mathrm{M}^{-1} \mathrm{~s}^{-1}$}
\end{tabular}

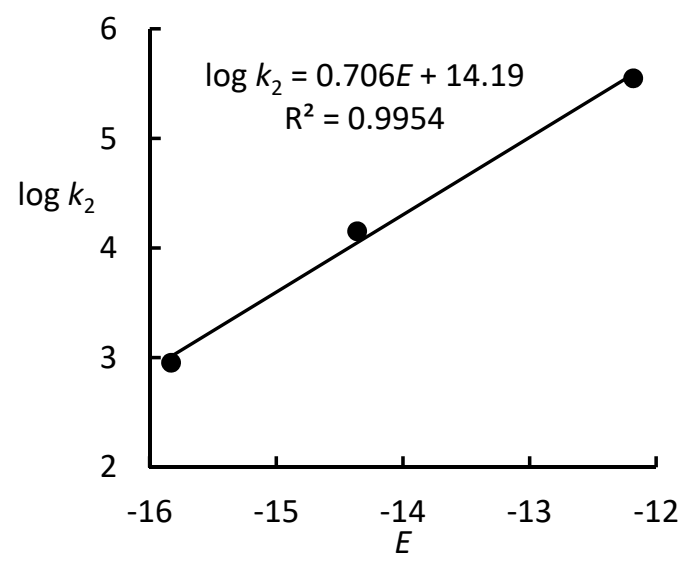

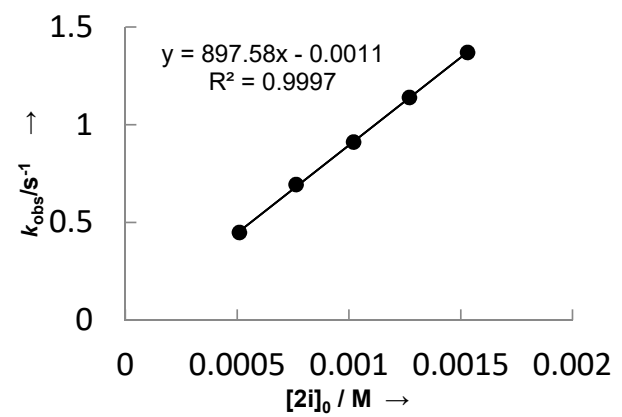

Figure S11. Correlation of $\log k_{2}$ for the reactions of carbanion $\mathbf{2 i}$ with reference electrophiles 10 vs the electrophilicity parameters $E$ of $\mathbf{1 0 .}$

For 2c (in $\mathrm{MeCN}): N=20.10, s \mathrm{~N}=0.71$ 
Table S45. Kinetics of the reactions of $\mathbf{2} \mathbf{k}$ with $\mathbf{1 0 d}$ in $\mathrm{CH}_{3} \mathrm{CN}$ at $20^{\circ} \mathrm{C}$ (deprotonated with $1.00-1.05$ equiv. $\mathrm{KO} t \mathrm{Bu}$, conventional $\mathrm{UV}-\mathrm{Vis}$ spectrometer, $\lambda=420 \mathrm{~nm}$ ).

\begin{tabular}{ccc}
{$[\mathbf{1 0 d}] / \mathbf{M}$} & {$[\mathbf{2 k}] / \mathbf{M}$} & $\boldsymbol{k}_{\text {obs }} / \mathbf{s}^{-1}$ \\
$2.50 \times 10^{-5}$ & $4.91 \times 10^{-4}$ & $4.96 \times 10^{1}$ \\
$2.50 \times 10^{-5}$ & $7.36 \times 10^{-4}$ & $7.28 \times 10^{1}$ \\
$2.50 \times 10^{-5}$ & $9.82 \times 10^{-4}$ & $1.03 \times 10^{2}$ \\
$2.50 \times 10^{-5}$ & $1.23 \times 10^{-3}$ & $1.32 \times 10^{2}$ \\
$2.50 \times 10^{-5}$ & $1.47 \times 10^{-3}$ & $1.56 \times 10^{2}$ \\
\hline \multicolumn{2}{c}{$k_{2}=(1.11 \pm 0.03) \times 10^{5} \mathrm{M}^{-1} \mathrm{~s}^{-1}$}
\end{tabular}

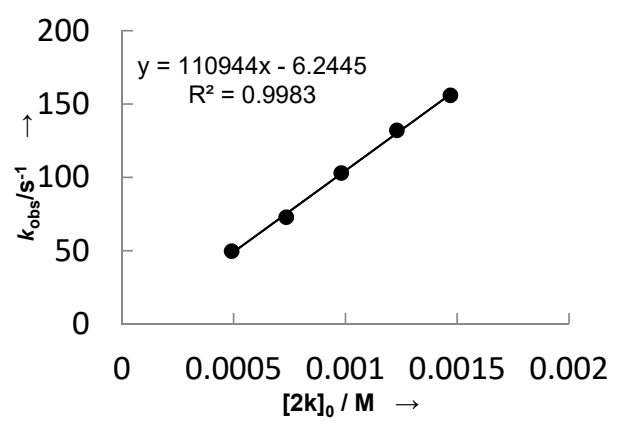

Table S46. Kinetics of the reactions of $\mathbf{2 k}$ with $\mathbf{1 0 f}$ in $\mathrm{CH}_{3} \mathrm{CN}$ at $20{ }^{\circ} \mathrm{C}$ (deprotonated with $1.00-1.05$ equiv. $\mathrm{KO} t \mathrm{Bu}$, conventional $\mathrm{UV}-\mathrm{Vis}$ spectrometer, $\lambda=374 \mathrm{~nm}$ ).

\begin{tabular}{ccc}
{$[\mathbf{1 0 f}] / \mathbf{M}$} & {$[\mathbf{2 k}] / \mathbf{M}$} & $\boldsymbol{k}_{\mathbf{o b s}} / \mathbf{s}^{-1}$ \\
$2.50 \times 10^{-5}$ & $5.00 \times 10^{-4}$ & 3.06 \\
$2.50 \times 10^{-5}$ & $7.49 \times 10^{-4}$ & 4.70 \\
$2.50 \times 10^{-5}$ & $9.99 \times 10^{-4}$ & 6.41 \\
$2.50 \times 10^{-5}$ & $1.25 \times 10^{-3}$ & 8.57 \\
$2.50 \times 10^{-5}$ & $1.50 \times 10^{-3}$ & 9.86 \\
\hline \multicolumn{2}{c}{$k_{2}=(6.99 \pm 0.26) \times 10^{3} \mathrm{M}^{-1} \mathrm{~s}^{-1}$}
\end{tabular}

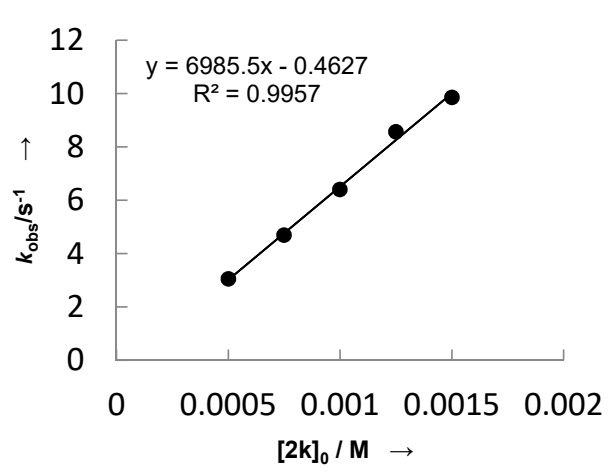

Table S47. Kinetics of the reactions of $2 \mathbf{k}$ with $\mathbf{1 0 g}$ in $\mathrm{CH}_{3} \mathrm{CN}$ at $20{ }^{\circ} \mathrm{C}$ (deprotonated with $1.00-1.05$ equiv. $\mathrm{KO} t \mathrm{Bu}$, conventional $\mathrm{UV}-\mathrm{Vis}$ spectrometer, $\lambda=380 \mathrm{~nm}$ ).

\begin{tabular}{cccc}
{$[\mathbf{1 0 g}] / \mathrm{M}$} & {$[\mathbf{2 k}] / \mathrm{M}$} & {$[18-\mathrm{c}-6] / \mathrm{M}$} & $k_{\text {obs }} / \mathrm{s}^{-1}$ \\
$2.50 \times 10^{-5}$ & $5.00 \times 10^{-4}$ & & $1.74 \times 10^{-1}$ \\
$2.50 \times 10^{-5}$ & $7.49 \times 10^{-4}$ & & $2.72 \times 10^{-1}$ \\
$2.50 \times 10^{-5}$ & $9.99 \times 10^{-4}$ & & $3.72 \times 10^{-1}$ \\
$2.50 \times 10^{-5}$ & $1.25 \times 10^{-3}$ & & $4.67 \times 10^{-1}$ \\
$\left(2.50 \times 10^{-5}\right.$ & $5.00 \times 10^{-4}$ & $1.03 \times 10^{-3}$ & $\left.1.64 \times 10^{-1}\right)$ \\
$\left(2.50 \times 10^{-5}\right.$ & $7.49 \times 10^{-4}$ & $1.55 \times 10^{-3}$ & $\left.2.64 \times 10^{-1}\right)$ \\
\hline \multicolumn{3}{c}{$k_{2}=(3.92 \pm 0.03) \times 10^{2} \mathrm{M}^{-1} \mathrm{~s}^{-1}$}
\end{tabular}
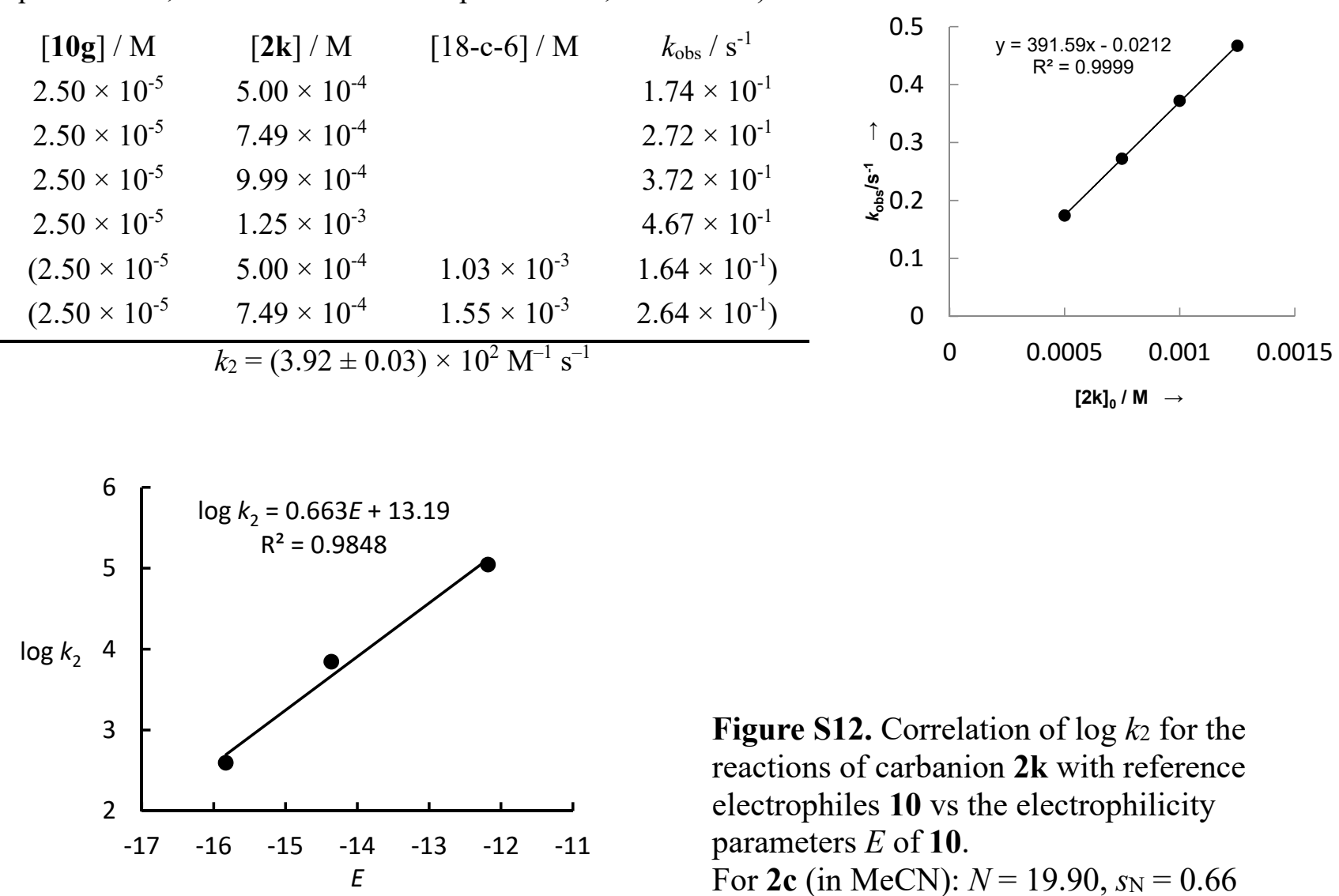

Figure S12. Correlation of $\log k_{2}$ for the reactions of carbanion $\mathbf{2} \mathbf{k}$ with reference electrophiles 10 vs the electrophilicity parameters $E$ of $\mathbf{1 0 .}$

For $2 \mathrm{c}($ in $\mathrm{MeCN}): N=19.90, s_{\mathrm{N}}=0.66$ 
6. Determination of $E$ for 1a and 1c
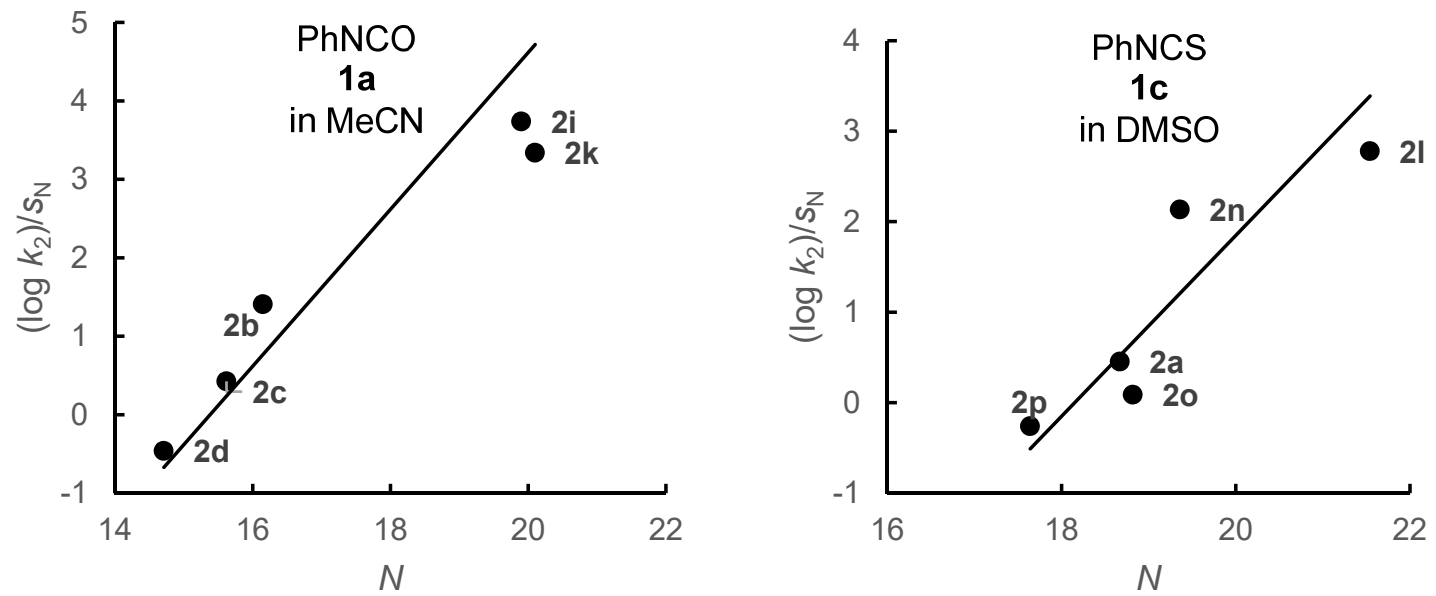

Figure S13. Plot of $\left(\log k_{2}\right) / s_{\mathrm{N}}$ vs $N$ for the reactions of carbanions 2 with phenyl isocyanate 1 a in acetonitrile and with phenyl isothiocyanate $1 \mathrm{c}$ in DMSO at $20^{\circ} \mathrm{C}$ (the slope is enforced to $1)$. 


\section{Procedure for Preparing $\mathrm{CO}_{2}$ Solutions in DMSO}

(a) Solubility of $\mathrm{CO}_{2}$ in DMSO at $20^{\circ} \mathrm{C}$ under $1 \mathrm{~atm}(101 \mathrm{kPa}) \mathrm{CO}_{2}$ partial pressure

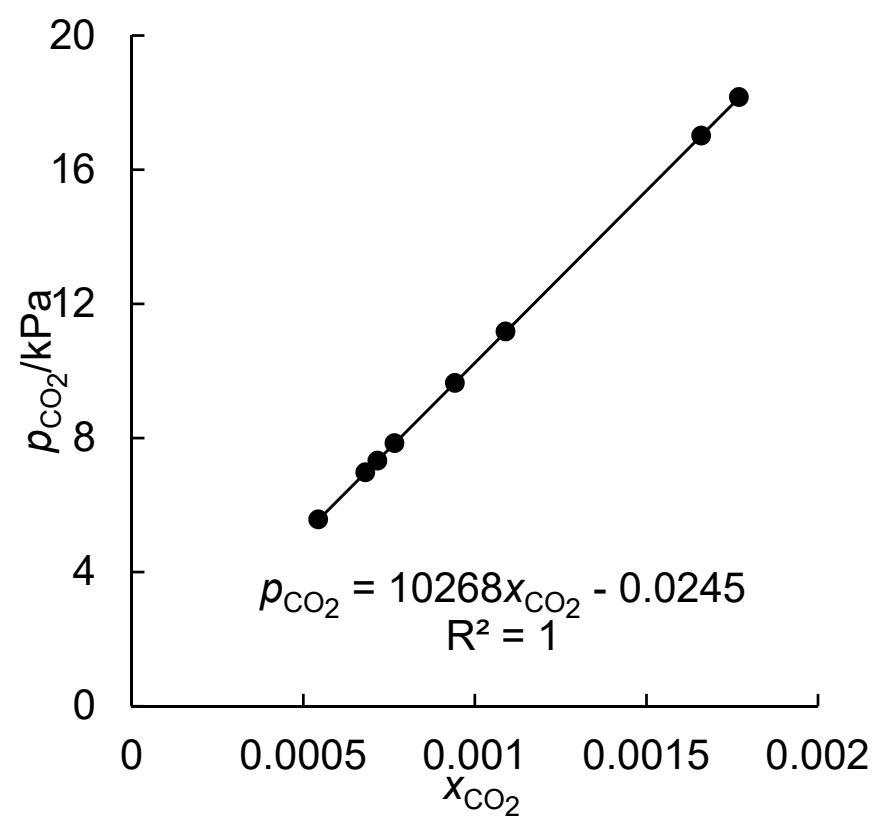

Figure S14. Plot of partial pressure of $\mathrm{CO}_{2}\left(p_{\mathrm{CO}_{2}}\right.$ in $\left.\mathrm{kPa}\right)$ with mole fraction of $\mathrm{CO}_{2}$ in DMSO solution $\left(x_{\mathrm{CO}_{2}}\right)$ at $20^{\circ} \mathrm{C}$ with data from ref $\mathrm{S} 17$.

Acorrelation between the partial pressure of $\mathrm{CO}_{2}$ in the gas phase and the solubility of $\mathrm{CO}_{2}$ in DMSO solution at $20{ }^{\circ} \mathrm{C}$ (Figure S14) was previously published in lit. ${ }^{\mathrm{S} 17}$ This correlation allows to calculate the molar fration of $\mathrm{CO}_{2}$ in DMSO solution when $p_{\mathrm{CO}_{2}}$ is 1 atm $(101 \mathrm{kPa})$.

$101 \mathrm{kPa}=10268 x \mathrm{CO}_{2}-0.0245$

Rearranges to $x_{\mathrm{CO}_{2}}=(101-0.0245) / 10268=9.83 \times 10^{-3}=n_{\mathrm{CO}_{2}} /\left(n_{\mathrm{CO}_{2}}+n_{\mathrm{DMSO}}\right)$

$n \mathrm{CO}_{2}$ is the molar amount of $\mathrm{CO}_{2}$ dissolved in DMSO solution and $n$ DMso corresponds to the molar amount of the DMSO liquid phase. Under the assumption that $n \mathrm{CO}_{2}$ is much smaller than $n$ DMSO, the relation simplifies to $x \mathrm{CO}_{2}=n n_{\mathrm{CO}_{2}} / n$ DMSO.

We calculated $n_{\text {DMSO }}$ for a given DMSO volume $V$ by the relation $n_{\text {DMSO }}=V d / M_{\text {DMSO }}$ using the molar mass $(M \mathrm{DMSO}=78.13 \mathrm{~g} / \mathrm{mol})$ and the density $\left(d=1100 \mathrm{~g} / \mathrm{L}\right.$ at $\left.20^{\circ} \mathrm{C}\right)$ of DMSO. In this way the concentration of $\mathrm{CO}_{2}$ in DMSO at $20{ }^{\circ} \mathrm{C}$ in equilibrium with an atmosphere of $\mathrm{CO}_{2}\left(\right.$ at $\left.p \mathrm{CO}_{2}=101 \mathrm{kPa}\right)$ is given by $\left[\mathrm{CO}_{2}\right]=n_{\mathrm{CO}_{2}} / V=0.138 \mathrm{M}$ (= concentration of the $\mathrm{CO}_{2}$ stock solution). 
(b) Procedure for preparing DMSO stock solutions with $\left[\mathrm{CO}_{2}\right]_{\mathrm{s}}=0.138 \mathrm{M}$

(Step 1) A 25-mL volumetric flask with rubber septum was partially evacuated and dried by using a heat gun. After further evacuation, the volumetric flask was filled with pure $\mathrm{CO}_{2}$ (sublimed dry ice passed through a short column filled with $\mathrm{CaCl}_{2}$ and silica gel). This operation was repeated three times.

(Step 2) By using a gas-tight syringe, anhydrous DMSO $(10 \mathrm{~mL})$ was added into a $25-\mathrm{mL}$ volumetric flask filled with $\mathrm{CO}_{2}$ (from Step 1). Then a stream of pure $\mathrm{CO}_{2}$ was passed through the DMSO solution for 30 min to generate a saturated $\mathrm{CO}_{2}$ solution (Figure S15).

(Step 3) A balloon with pure $\mathrm{CO}_{2}$ gas was placed on the flask to maintain a pressure of $1 \mathrm{~atm}$ $\mathrm{CO}_{2}$.
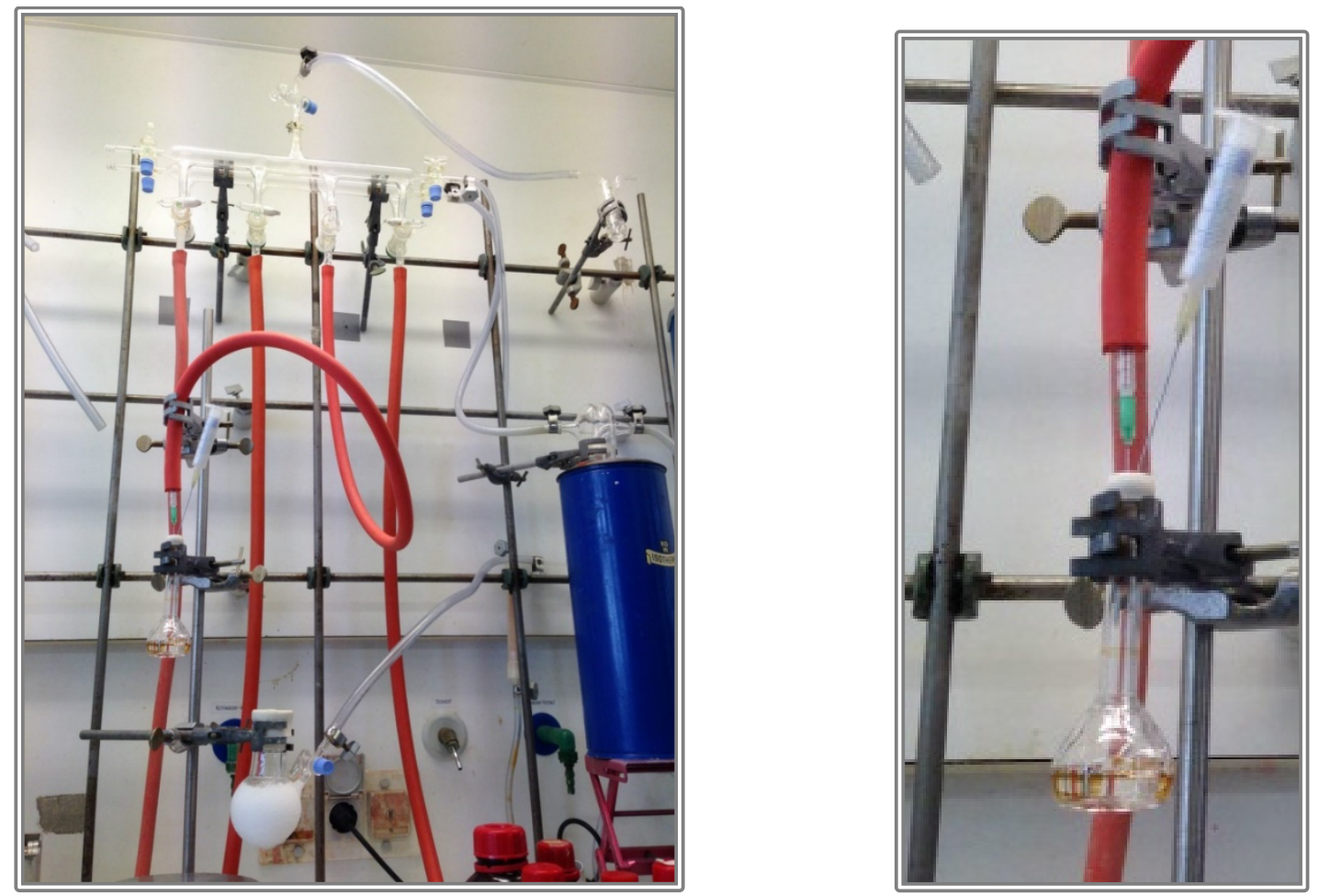

Figure S15. Photos taken during the preparing of the $\mathrm{CO}_{2}$ saturated DMSO solution (at 1 atm $\left.\mathrm{CO}_{2}\right)$.

(c) Procedure for preparing of a series of dilute solutions with different $\mathrm{CO}_{2}$ concentrations

(Step 4) Six 5-mL volumetric flasks with rubber septum (under dry Ar) were filled with anhydrous DMSO $(6.0 \mathrm{~mL})$ such that the head space above the liquid was less than $1 \mathrm{~mL}$ (Figure S16). Variable amounts ( $V$ from 0.06 to $0.18 \mathrm{~mL}$ ) of $\mathrm{CO}_{2}$ stock solution 
were transferred into the volumetric flasks to achieve dilute $\mathrm{CO}_{2}$ solutions in DMSO which were used for the kinetic measurements.

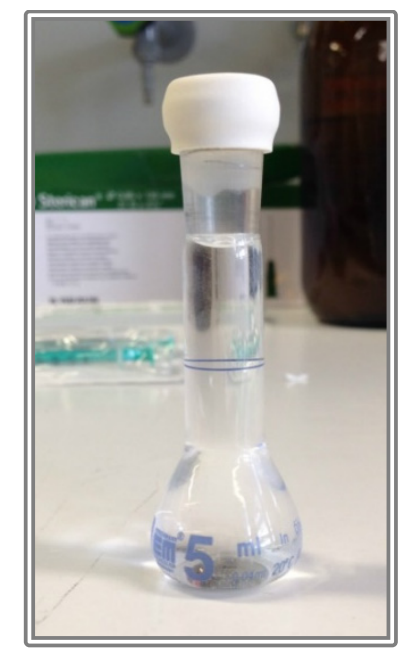

Figure S16. A 5-mL volumetric flask filled with ca $6 \mathrm{~mL}$ of DMSO

Given the small volume in the head space, it is justified to assume that most of the transferred $\mathrm{CO}_{2}$ in the 5-mL volumetric flask remains dissolved in DMSO. ${ }^{*}$ Hence, we derived the concentration of a dilute solution by the relation $\left[\mathrm{CO}_{2}\right]=V\left[\mathrm{CO}_{2}\right]_{\text {stock }} /(V+6.0 \mathrm{~mL})$.

(d) Titration of the $\mathrm{CO}_{2}$ content of DMSO solutions

To test whether the procedure for calculating the $\mathrm{CO}_{2}$ concentrations in dilute DMSO solutions holds, the content of $\mathrm{CO}_{2}$ in typical samples for the kinetic measurements was determined by tritration with aq $\mathrm{NaOH}$. Degassed water (Ar flow through distilled water for $1 \mathrm{~h}$ to remove dissolved $\mathrm{CO}_{2}$ ) was used for the titration. The titration was performed in a closed system to ensure that atmospheric $\mathrm{CO}_{2}$ does not influence the results.

To meet the conditions of the in the kinetic experiments, a small volume $(0.18 \mathrm{~mL})$ of the $\mathrm{CO}_{2}$ stock solution $\left(\left[\mathrm{CO}_{2}\right]=0.138 \mathrm{M}\right)$ was transferred into $6 \mathrm{~mL}$ of DMSO in a $5 \mathrm{~mL}$ volumetric flask. Then a $4 \mathrm{~mL}$ portion of this solution was mixed with $10 \mathrm{~mL}$ degassed water. The resulting solution was titrated with aq $\mathrm{NaOH}$ standard solution $(0.1 \mathrm{M})$ using phenolphthalein as indicator (7.4\%). The titrations gave an averaged $n \mathrm{CO}_{2}=0.017 \mathrm{mmol}$ (Table S48).

\footnotetext{
${ }^{*}$ A ratio of $>20$ for $n_{\mathrm{CO}_{2}}$ (in DMSO) $/ n_{\mathrm{CO}_{2}}$ (in gas phase) can be calculated under our conditions by applying the ideal gas law and the Henry law.
} 
Applying the ideal gas law and the Henry law we calculate the molar amount of $\mathrm{CO}_{2}, n \mathrm{CO}_{2}$, in the diluted DMSO solution to be

$$
n_{\mathrm{CO}_{2}}=[0.18 \mathrm{~mL} \times 0.138 \mathrm{M} /(0.18+6) \mathrm{mL}] \times 4 \mathrm{~mL}=0.0161 \mathrm{mmol} .
$$

Given that the $n \mathrm{CO}_{2}$ determined by the different methods agreed within $5.5 \%$ we used steps (1) to (4) to prepare $\mathrm{CO}_{2}$ solutions in DMSO and calculated the $\mathrm{CO}_{2}$ concentrations of the individual solutions by using the ideal gas law and the Henry law.

Table S48. Titration Experiment Results and the Obtained Average Value

\begin{tabular}{cc} 
Consumed $V(\mathrm{NaOH})(\mathrm{mL})$ & $n \mathrm{CO}_{2}(\mathrm{mmol})$ \\
0.16 & 0.016 \\
0.17 & 0.017 \\
0.18 & 0.018 \\
\hline average & 0.017
\end{tabular}




\section{Computational Details}

Initially, all structures were subjected to a conformational search with the OPLS3 ${ }^{\mathrm{S} 18}$ force field as implemented in MacroModel $^{\mathrm{S} 19}$ with the $\mathrm{MCMM}$ method in gas phase. All subsequent quantum-chemical calculations were performed with the Gaussian 16 A.03 set of codes. ${ }^{\text {S20 }}$

\section{(1) Methyl Anion Affinities}

Methyl anion affinities (MAA) were calculated following the previously published protocol ${ }^{\mathrm{S} 21}$ as the negative free energy for the methyl anion addition reaction (equation S1).

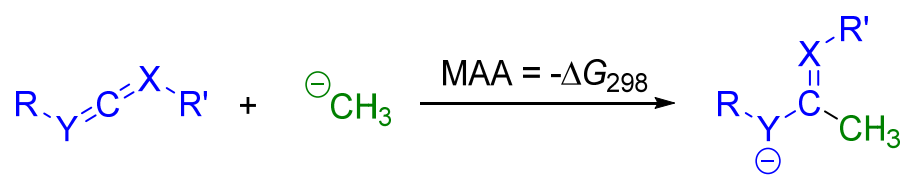

In short, after a conformational search, all structures were optimized in gas phase at the B3LYP/6-31G(d,p) level. Thermochemical corrections to Gibbs energies (Coor. $\Delta G$ ) at 298.15 $\mathrm{K}$ were subsequently calculated at the same level. For the inclusion of solvation effects, single point solvation corrections $\left(\Delta G_{\mathrm{sol}}\right)$ to $\Delta G_{298}$ were determined at the B3LYP/6-31G(d,p) by inclusion of the SMD solvation model for DMSO. ${ }^{\mathrm{S} 22}$ Additional single point calculations in gas phase were performed at the B3LYP/6-311++G(3df,2pd) level. Final Gibbs energies $\left(\Delta G_{298}\right.$, B3LYP/6-311++G(3df,2pd)//B3LYP/6-31G(d,p)) were calculated by combining these single point energies with the thermochemical corrections to Gibbs energies (Coor. $\Delta G$ ) calculated at a lower level and the solvation corrections $\left(\Delta G_{\mathrm{sol}}\right)$. For the calculation of the MAA energies, as in the previous procedures, ${ }^{\mathrm{S} 21}$ only the lowest lying conformers of each reaction were considered.

In analogy to previous studies, we tested the applicability of frontier molecular orbital energies as well as electrophilicity indices like electronic chemical potential $(\mu)$, chemical hardness $(\eta)$ and the global electrophilicity index $(\omega)$ to correlate with experimental electrophilicity parameters.

$$
\begin{gathered}
\mu \approx \frac{\mathrm{E}_{\mathrm{HOMO}}+\mathrm{E}_{\mathrm{LUMO}}}{2} \\
\eta \approx \mathrm{E}_{\mathrm{LUMO}}-\mathrm{E}_{\mathrm{HOMO}} \\
\omega=\frac{\mu^{2}}{2 \eta}(\mathrm{S} 4)
\end{gathered}
$$


Moreover, local electrophilicities were calculated based on the nucleophilic Fukui function $\left(f_{k}^{+}\right)$as defined in equation $\mathrm{S} 5$,

$$
\omega_{k}=\omega f_{k}^{+}
$$

where we used Yang and Mortier's method ${ }^{\mathrm{S} 23}$ in which $f_{k}^{+}$is defined as change of partial charge $q$ at the reaction center $k$ by adding an electron to the molecule with $N$ being the total number of electrons in the neutral species (S6). In this work, we applied Mulliken charges for this procedure.

$$
f_{k}^{+}=q(k, N+1)-q(k, N)
$$

\section{(2) PES calculations}

All structures were optimized at the B3LYP-D3/6-311+g(d,p $)^{\mathrm{S} 24}$ level of theory considering solvation by the IEFPCM model for $\mathrm{DMSO}^{\mathrm{S} 25}$ and dispersion corrections by the method of Grimme. ${ }^{\text {S26 }}$ Thermal corrections at $298 \mathrm{~K}$ were obtained at the same level of theory from vibrational frequencies and are unscaled. Transition states were validated by the presence of one imaginary frequency and IRC calculations. All conformers were Boltzmann weighted. Finally, a free energy correction $+7.91 \mathrm{~kJ} \mathrm{~mol}^{-1}$ was applied to all free energies to consider the conversion from gas phase $(1 \mathrm{~atm})$ to liquid phase $(1 \mathrm{M})$.

Distortion interaction analyses (DIA) were performed based on IRC calculations of the transition state $(\operatorname{maxpoint}=100$, recalc $=3)$ with the help of the AutoDIAS tool ${ }^{\mathrm{S} 27}$ following the method of Bickelhaupt and Houk. ${ }^{\text {S28 }}$ 
Table S49: Calculation of methyl anion affinities.

\begin{tabular}{|c|c|c|c|c|c|c|c|c|}
\hline & $\begin{array}{l}\mathrm{E}_{\text {tot }}(\mathrm{B} 3 \mathrm{LYP} / 6- \\
31 \mathrm{G}(\mathrm{d}, \mathrm{p}))\end{array}$ & $\begin{array}{l}\Delta \mathrm{G}(\mathrm{B} 3 \mathrm{LYP} / 6- \\
31 \mathrm{G}(\mathrm{d}, \mathrm{p}))\end{array}$ & $\begin{array}{l}\mathrm{E}_{\text {tot }}(\mathrm{SMD}(\mathrm{DMSO}) / / \\
\text { B3LYP/6-31G(d,p)) }\end{array}$ & $\begin{array}{l}\mathrm{E}_{\text {tot }}(\mathrm{B} 3 \mathrm{LYP} / \\
6-311++\mathrm{G} \\
(3 \mathrm{df}, 2 \mathrm{pd}))\end{array}$ & Coor. $\Delta \mathrm{G}$ & $\Delta \mathrm{Gsol}$ & $\begin{array}{l}\Delta \mathrm{G}_{\mathrm{gas}}(\mathrm{B} 3 \mathrm{LYP} / \\
6- \\
311++\mathrm{G}(3 \mathrm{df}, 2 \mathrm{pd}) / / \\
\mathrm{B} 3 \mathrm{LYP} / 6-31 \mathrm{G}(\mathrm{d}, \mathrm{p}))\end{array}$ & $\begin{array}{l}\Delta \mathrm{G}_{\text {DMSO }} \\
(\mathrm{SMD}(\mathrm{DMSO}) / / \\
\mathrm{B} 3 \mathrm{LYP} / 6-311++\mathrm{G} \\
(3 \mathrm{df}, 2 \mathrm{pd}) / / \\
\mathrm{B} 3 \mathrm{LYP} / 6-31 \mathrm{G}(\mathrm{d}, \mathrm{p}))\end{array}$ \\
\hline methylanion_zipse & -39.796028 & -39.787379 & -39.921582 & -39.856638 & 0.008649 & -0.125554 & -39.847989 & -39.973543 \\
\hline $\begin{array}{l}\text { REDO_1a_1 } \\
\text { REDO_1a_me_z_1 }\end{array}$ & $\begin{array}{l}-399.740423 \\
-439.696804\end{array}$ & $\begin{array}{l}-399.668907 \\
-439.590467\end{array}$ & $\begin{array}{l}-399.747265 \\
-439.780577\end{array}$ & $\begin{array}{l}-399.873713 \\
-439.855937\end{array}$ & $\begin{array}{l}0.071516 \\
0.106337\end{array}$ & $\begin{array}{l}-0.006842 \\
-0.083773\end{array}$ & $\begin{array}{l}-399.802197 \\
-439.749600 \\
\mathrm{MAA}_{\text {gas }}=-261.0\end{array}$ & $\begin{array}{l}-399.809039 \\
-439.833373 \\
\mathrm{MAA}_{\text {solv }}=-133.4\end{array}$ \\
\hline $\begin{array}{l}\text { REDO_1b_1 } \\
\text { REDO_1b_me_z_4_2 }\end{array}$ & $\begin{array}{l}-987.621593 \\
-1027.608403\end{array}$ & $\begin{array}{l}-987.522286 \\
-1027.475066\end{array}$ & $\begin{array}{l}-987.634878 \\
-1027.697525\end{array}$ & $\begin{array}{l}-987.899807 \\
-1027.908057\end{array}$ & $\begin{array}{l}0.099307 \\
0.133337\end{array}$ & $\begin{array}{l}-0.013285 \\
-0.089122\end{array}$ & $\begin{array}{l}-987.800500 \\
-1027.774720 \\
\mathrm{MAA}_{\text {gas }}=-331.4\end{array}$ & $\begin{array}{l}-987.813784 \\
-1027.863842 \\
\mathrm{MAA}_{\text {solv }}=-200.9\end{array}$ \\
\hline $\begin{array}{l}\text { REDO_1c_1 } \\
\text { REDO_1c_me_e_2 }\end{array}$ & $\begin{array}{l}-722.701586 \\
-762.668733\end{array}$ & $\begin{array}{l}-722.633778 \\
-762.565578\end{array}$ & $\begin{array}{l}-722.708347 \\
-762.752421\end{array}$ & $\begin{array}{l}-722.834820 \\
-762.823307\end{array}$ & $\begin{array}{l}0.067808 \\
0.103155\end{array}$ & $\begin{array}{l}-0.006761 \\
-0.083688\end{array}$ & $\begin{array}{l}-722.767012 \\
-762.720152 \\
\text { MAA }_{\text {gas }}=-276.1\end{array}$ & $\begin{array}{l}-722.773773 \\
-762.803840 \\
\mathrm{MAA}_{\text {solv }}=-148.4\end{array}$ \\
\hline $\begin{array}{l}\text { REDO_1d_1 } \\
\text { s_1d_o_me_z_2 }\end{array}$ & $\begin{array}{l}-927.199579 \\
-967.193688\end{array}$ & $\begin{array}{l}-927.133315 \\
-967.091349\end{array}$ & $\begin{array}{l}-927.209222 \\
-967.263999\end{array}$ & $\begin{array}{l}-927.411120 \\
-967.425551\end{array}$ & $\begin{array}{l}0.066264 \\
0.102339\end{array}$ & $\begin{array}{l}-0.009644 \\
-0.070312\end{array}$ & $\begin{array}{l}-927.344856 \\
-967.323212 \\
\text { MAA }_{\text {gas }}=-342.3\end{array}$ & $\begin{array}{l}-927.354499 \\
-967.393524 \\
\text { MAA }_{\text {solv }}=-171.9\end{array}$ \\
\hline $\begin{array}{l}\text { REDO_1e_1 } \\
\text { REDO_1e_me_z_1 }\end{array}$ & $\begin{array}{l}-610.904191 \\
-650.872855\end{array}$ & $\begin{array}{l}-610.749086 \\
-650.681333\end{array}$ & $\begin{array}{l}-610.917905 \\
-650.954238\end{array}$ & $\begin{array}{l}-611.091699 \\
-651.083378\end{array}$ & $\begin{array}{l}0.155105 \\
0.191522\end{array}$ & $\begin{array}{l}-0.013714 \\
-0.081383\end{array}$ & $\begin{array}{l}-610.936594 \\
-650.891856 \\
\text { MAA }_{\text {gas }}=-281.6\end{array}$ & $\begin{array}{l}-610.950308 \\
-650.973238 \\
\mathrm{MAA}_{\text {solv }}=-129.7\end{array}$ \\
\hline $\begin{array}{l}\text { REDO_1f_11 } \\
\text { REDO_1f_me_z_3 }\end{array}$ & $\begin{array}{l}-618.169762 \\
-658.107634\end{array}$ & $\begin{array}{l}-617.877240 \\
-657.779659\end{array}$ & $\begin{array}{l}-618.183470 \\
-658.194177\end{array}$ & $\begin{array}{l}-618.346559 \\
-658.308045\end{array}$ & $\begin{array}{l}0.292522 \\
0.327975\end{array}$ & $\begin{array}{l}-0.013708 \\
-0.086544\end{array}$ & $\begin{array}{l}-618.054037 \\
-657.980070 \\
\mathrm{MAA}_{\text {gas }}=-204.9\end{array}$ & $\begin{array}{l}-618.067745 \\
-658.066614 \\
\text { MAA }_{\text {solv }}=-66.5\end{array}$ \\
\hline $\begin{array}{l}\text { REDO_1g_1 } \\
\text { REDO_1g_me_1 }\end{array}$ & $\begin{array}{l}-834.488868 \\
-874.454250\end{array}$ & $\begin{array}{l}-834.504871 \\
-874.439229\end{array}$ & $\begin{array}{l}-834.489036 \\
-874.541009\end{array}$ & $\begin{array}{l}-834.568429 \\
-874.552941\end{array}$ & $\begin{array}{l}-0.016003 \\
0.015021\end{array}$ & $\begin{array}{l}-0.000167 \\
-0.086759\end{array}$ & $\begin{array}{l}-834.584432 \\
-874.537920 \\
\mathrm{MAA}_{\text {gas }}=-277.0\end{array}$ & $\begin{array}{l}-834.584599 \\
-874.624679 \\
\text { MAA }_{\text {solv }}=-174.7\end{array}$ \\
\hline $\begin{array}{l}\text { REDO_1h_1 } \\
\text { REDO_1h_me_1 }\end{array}$ & $\begin{array}{l}-188.580939 \\
-228.502197\end{array}$ & $\begin{array}{l}-188.590066 \\
-228.481990\end{array}$ & $\begin{array}{l}-188.582579 \\
-228.594697\end{array}$ & $\begin{array}{l}-188.659994 \\
-228.617076\end{array}$ & $\begin{array}{l}-0.009127 \\
0.020207\end{array}$ & $\begin{array}{l}-0.001640 \\
-0.092500\end{array}$ & $\begin{array}{l}-188.669121 \\
-228.596869 \\
\text { MAA }_{\text {gas }}=-209.4\end{array}$ & $\begin{array}{l}-188.670761 \\
-228.689370 \\
\mathrm{MAA}_{\text {solv }}=-118.3\end{array}$ \\
\hline
\end{tabular}


Table S50: Quantum chemically calculate methyl anion affinities in gas phase and in DMSO solution as well as different common quantum chemically calculated electrophilicity descriptors.

\begin{tabular}{|c|c|c|c|c|c|c|c|c|c|c|c|c|c|c|}
\hline & & File & $E$ & $\begin{array}{l}\mathrm{MAA}_{\text {solv }} \\
\left(\mathrm{kJ} \mathrm{mol}^{-1}\right)\end{array}$ & $\begin{array}{l}\mathrm{MAA}_{\text {gas }} \\
\left(\mathrm{kJ} \mathrm{mol}^{-1}\right)\end{array}$ & $\begin{array}{l}\mathrm{E}_{\text {номо }} \\
\text { (Hartree) }\end{array}$ & $\begin{array}{l}\mathrm{E}_{\mathrm{LUMO}} \\
\text { (Hartree) }\end{array}$ & $\mu$ (Hartree) & $\eta$ (Hartree) & $\omega(\mathrm{eV})$ & $\begin{array}{l}\text { Mulliken } \\
\text { charge } \\
\text { (neutral) }\end{array}$ & $\begin{array}{l}\text { Mulliken } \\
\text { charge } \\
(+1)\end{array}$ & $\mathrm{f}_{\mathrm{k}}^{+}$ & $\omega_{\mathrm{k}}(\mathrm{eV})$ \\
\hline PhNCO & $1 \mathrm{a}$ & REDO_1a_1 & -15.37 & -133.4 & -261.0 & -0.23744 & -0.02371 & -0.130575 & 0.21373 & 1.09 & 0.599 & 0.442 & 0.157 & 0.17 \\
\hline TIC & $1 b$ & REDO_1b_1 & -7.69 & -200.9 & -331.4 & -0.27376 & -0.05875 & -0.166255 & 0.21501 & 1.75 & 0.650 & 0.516 & 0.134 & 0.23 \\
\hline PhNCS & 1c & REDO_1c_1 & -18.15 & -148.4 & -276.1 & -0.23182 & -0.04576 & -0.138790 & 0.18606 & 1.41 & 0.172 & 0.063 & 0.109 & 0.15 \\
\hline NO2PhNCS & 1d & REDO_1d_1 & -15.89 & -171.9 & -342.3 & -0.25644 & -0.10474 & -0.180590 & 0.15170 & 2.92 & 0.186 & 0.130 & 0.056 & 0.16 \\
\hline DPC & $1 e$ & REDO_1e-1 & -20.14 & -129.7 & -281.6 & -0.22394 & -0.02753 & -0.125735 & 0.19641 & 1.10 & 0.609 & 0.464 & 0.145 & 0.16 \\
\hline DCC & If & REDO_1f-11 & -- & -66.5 & -204.9 & -0.23978 & 0.03689 & -0.101445 & 0.27667 & 0.51 & 0.593 & 0.358 & 0.235 & 0.12 \\
\hline $\mathrm{CS} 2$ & $1 \mathrm{~g}$ & REDO_-1 ${ }^{-} \_1$ & -17.7 & -174.7 & -277.0 & -0.27776 & -0.07494 & -0.176350 & 0.20282 & 2.09 & -0.050 & -0.149 & 0.099 & 0.21 \\
\hline $\mathrm{CO} 2$ & $1 \mathrm{~h}$ & REDO_1h_1 & -16.3 & -118.3 & -209.4 & -0.36997 & 0.02992 & -0.170025 & 0.39989 & 0.98 & 0.719 & 0.164 & 0.555 & 0.55 \\
\hline
\end{tabular}


Correlations between Experimental Electrophilicity $E$ and Quantum-Chemically Calculated Properties

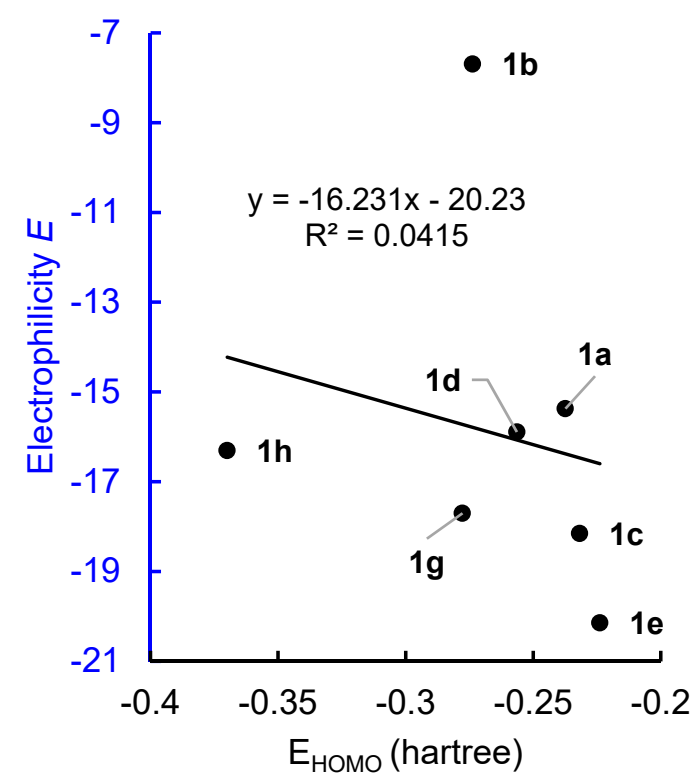

Figure S17. Correlation of electrophilicity parameters $E$ with HOMO energies $\mathrm{E}_{\text {HOMO. }}$

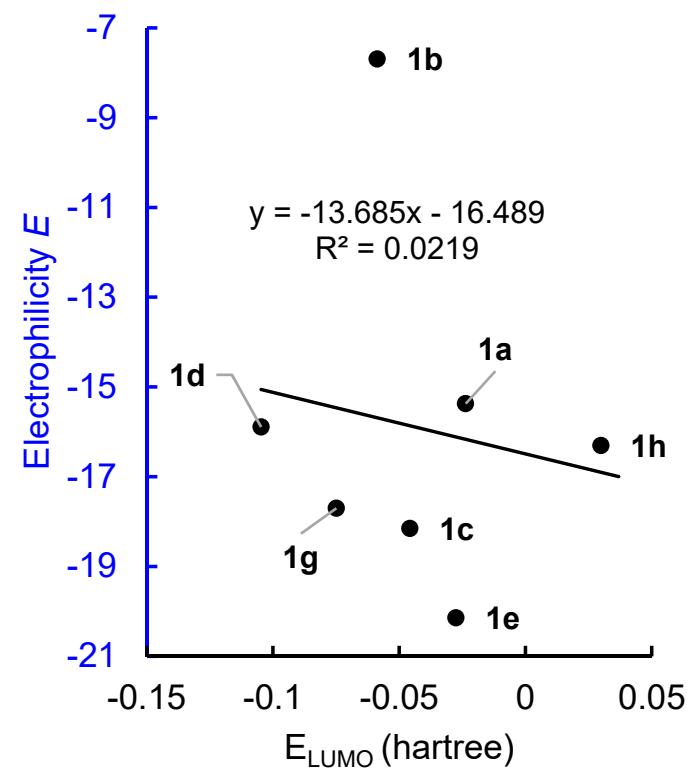

Figure S18. Correlation of electrophilicity parameters $E$ with LUMO energies $\mathrm{E}_{\text {LUMO. }}$

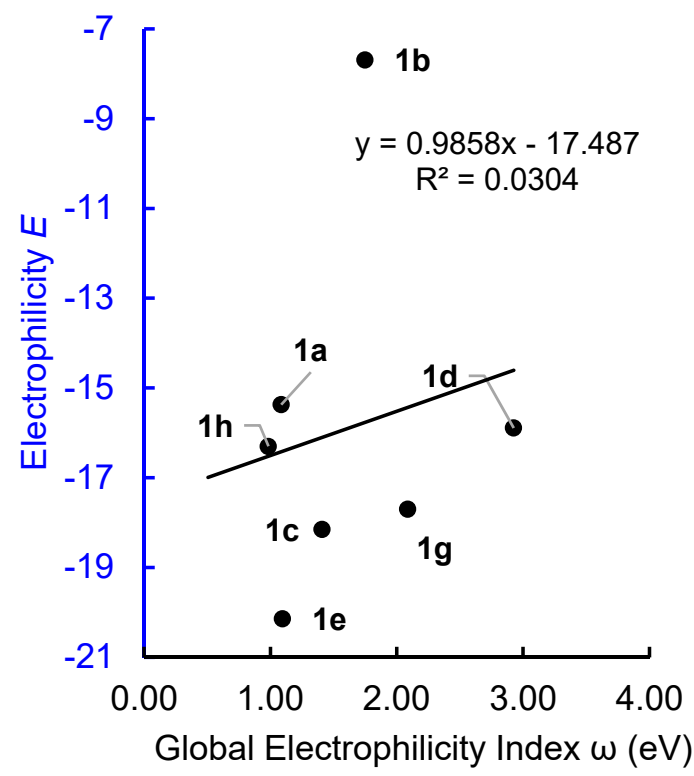

Figure S19. Correlation of electrophilicity parameters $E$ with global electrophilicity indices $\omega$. 


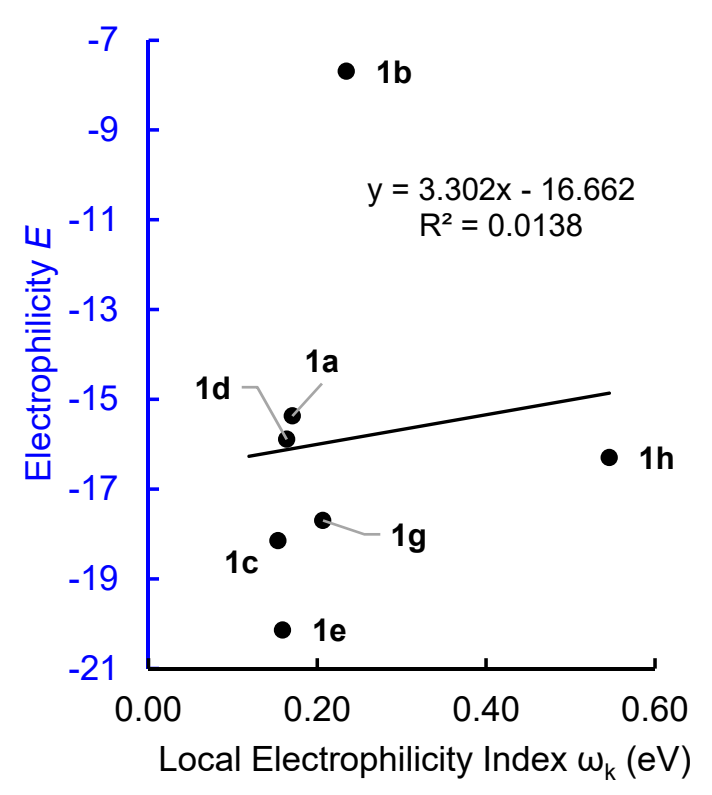

Figure S20. Correlation of electrophilicity parameters $E$ with global electrophilicity indices $\omega_{\mathrm{k}}$.
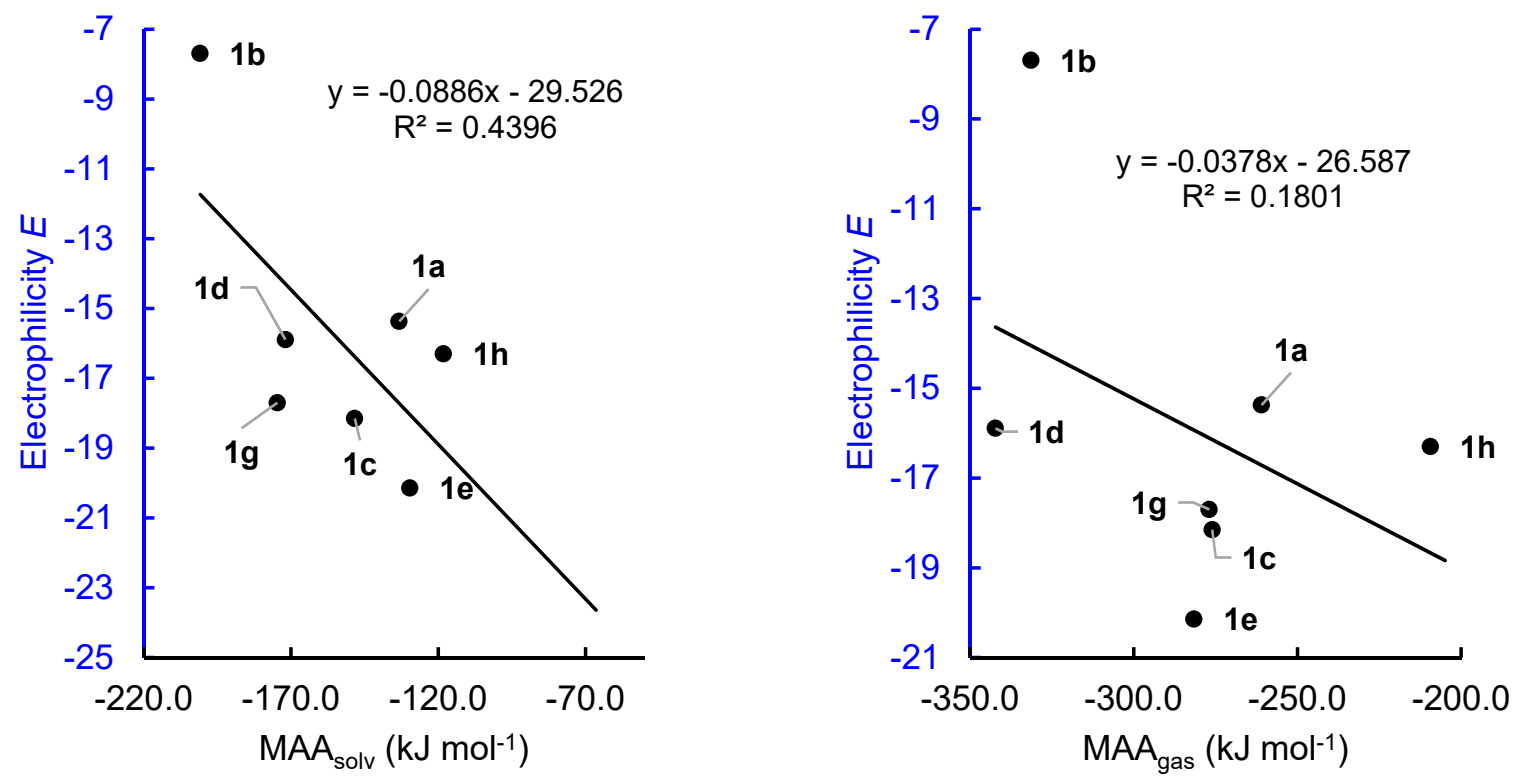

Figure S21. Correlation of electrophilicity parameters $E$ with solution corrected methyl anion affinities $\mathrm{MAA}_{\text {solv }}$

Figure S22. Correlation of electrophilicity parameters $E$ with gas phase methyl anion affinities $\mathrm{MAA}_{\text {solv }}$ 
Table S51: Quantum-chemically calculated activation and reaction energies for the reactions of heteroallenes 1a-h with nucleophile $\mathbf{2 a}$ at the IEFPCM(DMSO)/B3LYP-D3/6-311+G(d,p) level of theory.

\begin{tabular}{|c|c|c|c|c|c|}
\hline & Filename & SCF & G298 & Weighting & TS type \\
\hline $2 \mathbf{a}$ & REDO_tfs_anion_2 & -1156.953255 & -1156.865502 & & \\
\hline $1 \mathbf{a}$ & REDO_1a_1 & -399.856288 & -399.785537 & & \\
\hline TS & $\begin{array}{l}\text { REDO_phnco_tfs_1_ts1 } \\
\text { REDO_phnco_tfs_3 ts } 1 \\
\text { REDO_phnco_tfs_5_ts } 1 \\
\text { REDO_phnco_tfs_6_ts } 1 \\
\text { REDO_phnco_tfs_8_ts1 } \\
\text { o_phncs_tfs_3_ts1 } \\
\text { o_phncs_tfs_6_ts1 } \\
\text { o_phncs_tfs_8_ts1 } \\
\text { o_phncs_tfs_10_ts1 } \\
\text { o_phncs_tfs_10_ts1_r2 } \\
\text { Weighted }\end{array}$ & $\begin{array}{l}-1556.798078 \\
-1556.795087 \\
-1556.798764 \\
-1556.802735 \\
-1556.803302 \\
-1556.802659 \\
-1556.803463 \\
-1556.809654 \\
-1556.811758 \\
-1556.808218\end{array}$ & $\begin{array}{l}-1556.618930 \\
-1556.616830 \\
-1556.619643 \\
-1556.624828 \\
-1556.625651 \\
-1556.620424 \\
-1556.623103 \\
-1556.628128 \\
-1556.632638 \\
-1556.627404 \\
-1556.632574\end{array}$ & $\begin{array}{l}0.00000 \\
0.00000 \\
0.00000 \\
0.00025 \\
0.00060 \\
0.00000 \\
0.00004 \\
0.00828 \\
0.98699 \\
0.00384\end{array}$ & $\begin{array}{l}\text { anti } \\
\text { anti } \\
\text { anti } \\
\text { anti } \\
\text { anti } \\
\text { syn } \\
\text { syn } \\
\text { syn } \\
\text { syn } \\
\text { syn }\end{array}$ \\
\hline IA & $\begin{array}{l}\text { REDO_phnco_tfs_1 } \\
\text { REDO_phnco_tfs_2 } \\
\text { REDO_phnco_tfs_3 } \\
\text { REDO_phnco_tfs_4 } \\
\text { REDO_phnco_tfs_5 } \\
\text { REDO_phnco_tts_6 } \\
\text { REDO_phnco_tfs_7 } \\
\text { REDO_phnco_tfs_8 } \\
\text { REDO_phnco_tfs_9 } \\
\text { REDO_phncs_tfs_1 } \\
\text { REDO_phncs_tfs_2 } \\
\text { REDO_phncs_tfs_3 } \\
\text { REDO_phncs_tfs_4 } \\
\text { REDO_phncs_tfs_6 } \\
\text { REDO_phncs_tfs_8 } \\
\text { REDO_phncs_tfs_9 } \\
\text { REDO_phncs_tfs_10 } \\
\text { Weighted }\end{array}$ & $\begin{array}{l}-1556.832640 \\
-1556.832616 \\
-1556.833501 \\
-1556.830198 \\
-1556.829619 \\
-1556.833324 \\
-1556.833375 \\
-1556.834602 \\
-1556.832621 \\
-1556.833482 \\
-1556.833513 \\
-1556.820590 \\
-1556.832626 \\
-1556.829543 \\
-1556.821362 \\
-1556.832638 \\
-1556.831462\end{array}$ & $\begin{array}{l}-1556.649351 \\
-1556.648639 \\
-1556.649632 \\
-1556.647574 \\
-1556.646705 \\
-1556.650663 \\
-1556.650488 \\
-1556.651510 \\
-1556.648099 \\
-1556.650269 \\
-1556.650002 \\
-1556.635441 \\
-1556.648820 \\
-1556.645650 \\
-1556.636617 \\
-1556.649458 \\
-1556.647598 \\
-1556.650567\end{array}$ & $\begin{array}{l}0.03699 \\
0.01739 \\
0.04986 \\
0.00562 \\
0.00224 \\
0.14874 \\
0.12353 \\
0.36512 \\
0.00981 \\
0.09794 \\
0.07375 \\
0.00000 \\
0.02107 \\
0.00073 \\
0.00000 \\
0.04145 \\
0.00577\end{array}$ & $\begin{array}{l}\text { anti } \\
\text { anti } \\
\text { anti } \\
\text { anti } \\
\text { anti } \\
\text { anti } \\
\text { anti } \\
\text { anti } \\
\text { anti } \\
\text { anti } \\
\text { anti } \\
\text { syn } \\
\text { anti } \\
\text { syn } \\
\text { syn } \\
\text { anti } \\
\text { syn }\end{array}$ \\
\hline \multirow[t]{2}{*}{ TA } & $\begin{array}{l}\text { REDO_1a_nh_tfs_taut_1 } \\
\text { Weighted }\end{array}$ & -1556.845993 & $\begin{array}{l}-1556.661763 \\
-1556.661763\end{array}$ & 1.00000 & \\
\hline & & $\begin{array}{l}\Delta \mathrm{G}_{\text {calc }}^{\ddagger} \\
\Delta_{\mathrm{r}} \mathrm{G}_{\text {calc }}^{0}(\mathrm{IA}) \\
\Delta_{\mathrm{r}} \mathrm{G}_{\text {calc }}^{0}(\mathrm{TA})\end{array}$ & $\begin{array}{l}40.6 \\
-6.7 \\
-36.1\end{array}$ & $\begin{array}{l}\mathrm{kJ} / \mathrm{mol} \\
\mathrm{kJ} / \mathrm{mol} \\
\mathrm{kJ} / \mathrm{mol}\end{array}$ & \\
\hline
\end{tabular}

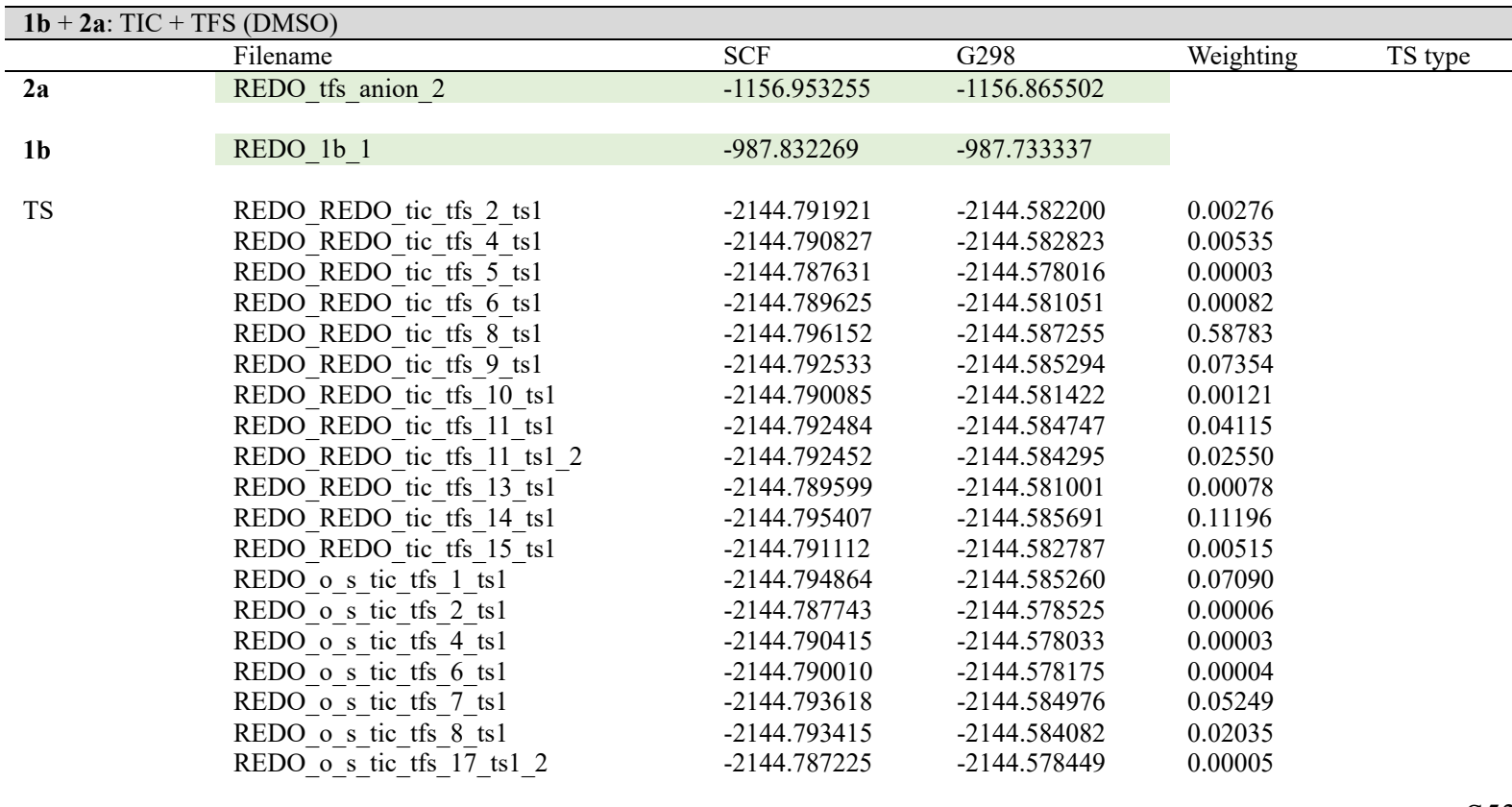


$-2144.834831$

$-2144.832227$

$-2144.833204$

$-2144.838676$

REDO tic -tfs-

REDO_tic tfs_8

REDO tic tfs 92

REDO tic tfs 10

REDO tic tfs 11

REDO tic tfs 12

REDO_tic_tfs_13

REDO tic tfs 14

REDO tic tfs 15

REDO_tic_tfs_16

REDO tic tfs 17

REDO_tic_tfs_18

REDO tic tfs 19

O_s_tic_tfs_1

o_s_tic_tfs_4

o s tic tfs 7

o_s_tic_tfs_8

o s tic tfs 9

o s tic tfs 10

o_s_tic_tfs_12

o s tic tfs 13

o_s_tic_tfs_14

o_s_tic tfs 17

o s tic tfs 19

o_s_tic_tfs_20

Weighted

REDO tic tfs $t$

REDO tic tfs t 4

REDO_tic_tfs_t 6

Weighted

$-2144.837774$

$-2144.833155$

$-2144.837370$

$-2144.837939$

$-2144.829332$

$-2144.835502$

$-2144.838941$

$-2144.836527$

$-2144.839103$

$-2144.839400$

$-2144.835891$

$-2144.838878$

$-2144.835778$

$-2144.836350$

$-2144.833881$

$-2144.836624$

$-2144.833873$

$-2144.836503$

$-2144.831998$

$-2144.833982$

$-2144.836273$

$-2144.822595$

$-2144.829879$

$-2144.836173$
TA

$\begin{array}{ll}-2144.621561 & 0.00225 \\ -2144.619389 & 0.00023 \\ -2144.620707 & 0.00091 \\ -2144.624446 & 0.04794 \\ -2144.626047 & 0.26180 \\ -2144.620854 & 0.00106 \\ -2144.624810 & 0.07055 \\ -2144.625203 & 0.10700 \\ -2144.616406 & 0.00001 \\ -2144.621825 & 0.00298 \\ -2144.624824 & 0.07163 \\ -2144.622203 & 0.00445 \\ -2144.624212 & 0.03743 \\ -2144.626090 & 0.27415 \\ -2144.622379 & 0.00536 \\ -2144.625187 & 0.10523 \\ -2144.621646 & 0.00246 \\ -2144.620797 & 0.00100 \\ -2144.620018 & 0.00044 \\ -2144.621279 & 0.00167 \\ -2144.619058 & 0.00016 \\ -2144.620183 & 0.00052 \\ -2144.618559 & 0.00009 \\ -2144.619064 & 0.00016 \\ -2144.619491 & 0.00025 \\ -2144.607584 & 0.00000 \\ -2144.616646 & 0.00001 \\ -2144.619435 & 0.00024 \\ -2144.625450 & \\ -2144.619170 & 0.27657 \\ -2144.620048 & 0.70158 \\ -2144.616776 & 0.02184 \\ -2144.619734 & \\ 24.9 & \mathrm{~kJ} / \mathrm{mol} \\ -77.8 & \mathrm{~kJ} / \mathrm{mol} \\ -62.8 & \\ & \end{array}$

\begin{tabular}{|c|c|c|c|c|c|}
\hline & Filename & SCF & G298 & Weighting & TS type \\
\hline $\mathbf{2 a}$ & REDO_tfs_anion_2 & -1156.953255 & -1156.865502 & & \\
\hline 1c & REDO_1c_1 & -722.815578 & -722.749237 & & \\
\hline \multirow[t]{11}{*}{ TS } & REDO_phncs_tfs_1_ts 1 & -1879.752727 & -1879.576662 & 0.00000 & anti \\
\hline & REDO_phncs_tfs_3_ts 1 & -1879.755772 & -1879.578019 & 0.00001 & syn \\
\hline & REDO_phncs_tfs_4_ts 1 & -1879.756366 & -1879.580367 & 0.00013 & anti \\
\hline & REDO_phncs_tfs_6_ts 1 & -1879.756660 & -1879.578637 & 0.00002 & syn \\
\hline & REDO_phncs_tfs_7_ts 1 & -1879.760729 & -1879.584721 & 0.01356 & anti \\
\hline & REDO_phncs_tfs_8_ts 1 & -1879.762969 & -1879.585404 & 0.02801 & syn \\
\hline & REDO_phncs_tfs_9_ts1 & -1879.751513 & -1879.576530 & 0.00000 & anti \\
\hline & REDO_phncs_tfs_10_ts 1 & -1879.765378 & -1879.588732 & 0.95404 & syn \\
\hline & s_phnco_tfs_1_ts 1 & -1879.755166 & -1879.579257 & 0.00004 & anti \\
\hline & s_phnco_tfs_6_ts 1 & -1879.759386 & -1879.583610 & 0.00418 & anti \\
\hline & Weighted & & -1879.588561 & & \\
\hline \multirow[t]{11}{*}{ IA } & REDO_phncs_tfs_1 & -1879.802263 & -1879.621694 & 0.35378 & anti \\
\hline & REDO_phncs_tfs_4 & -1879.801209 & -1879.619882 & 0.05180 & anti \\
\hline & REDO_phncs_tfs_5 & -1879.801079 & -1879.620540 & 0.10410 & anti \\
\hline & REDO_phncs_tfs_6 & -1879.790707 & -1879.610182 & 0.00000 & syn \\
\hline & REDO_phncs_tfs_7 & -1879.802287 & -1879.621985 & 0.48177 & anti \\
\hline & REDO_phncs_tfs_8 & -1879.786541 & -1879.604465 & 0.00000 & syn \\
\hline & REDO_phncs_tfs_9 & -1879.796421 & -1879.615044 & 0.00031 & anti \\
\hline & REDO_phncs_tfs_10 & -1879.793997 & -1879.612736 & 0.00003 & syn \\
\hline & REDO_phnco_tfs_4 & -1879.792050 & -1879.611547 & 0.00001 & anti \\
\hline & REDO_phnco_tfs_6 & -1879.797236 & -1879.618144 & 0.00821 & anti \\
\hline & Weighted & & -1879.621589 & & \\
\hline \multirow[t]{4}{*}{ TA } & REDO_1c_nh_tfs_taut_1 & -1879.801503 & -1879.621439 & 0.32226 & \\
\hline & REDO_1c_nh_tfs_taut_2 & -1879.802539 & -1879.622140 & 0.67774 & \\
\hline & Weighted & & -1879.621914 & & \\
\hline & & $\begin{array}{l}\Delta \mathrm{G}_{\text {calc }}^{\ddagger} \\
\Delta_{\mathrm{r}} \mathrm{G}_{\text {calc }}(\mathrm{IA})\end{array}$ & $\begin{array}{l}60.8 \\
-25.9\end{array}$ & $\begin{array}{l}\mathrm{kJ} / \mathrm{mol} \\
\mathrm{kJ} / \mathrm{mol}\end{array}$ & \\
\hline
\end{tabular}


$\Delta_{\mathrm{r}} \mathrm{G}_{\text {calc }}^{0}(\mathrm{TA})$

\begin{tabular}{|c|c|c|c|c|c|}
\hline & Filename & SCF & G298 & Weighting & TS type \\
\hline $2 \mathbf{a}$ & REDO_tfs_anion_2 & -1156.953255 & -1156.865502 & & \\
\hline 1d & REDO_1d_1 & -927.385765 & -927.321170 & & \\
\hline TS & $\begin{array}{l}\text { REDO_no2phncs_tfs_1_ts1 } \\
\text { REDO_no2phncs_tfs_2_ts1 } \\
\text { REDO_no2phncs_tfs_5_ts1 } \\
\text { REDO_no2phncs_tfs_6_ts1 } \\
\text { REDO_no2phncs_tfs_7_ts1 } \\
\text { REDO_no2phncs_tfs_9_ts1 } \\
\text { REDO_no2phncs_tfs_10_ts1 } \\
\text { REDO_no2phncs_tfs_9_ts1_r1 } \\
\text { REDO_no2phncs_tfs_9_ts1_r2 } \\
\text { Weighted }\end{array}$ & $\begin{array}{l}-2084.332325 \\
-2084.330759 \\
-2084.331713 \\
-2084.340518 \\
-2084.334985 \\
-2084.340249 \\
-2084.326069 \\
-2084.340599 \\
-2084.338060\end{array}$ & $\begin{array}{l}-2084.156111 \\
-2084.155948 \\
-2084.156257 \\
-2084.164682 \\
-2084.161442 \\
-2084.166079 \\
-2084.152523 \\
-2084.166852 \\
-2084.163192 \\
-2084.166436\end{array}$ & $\begin{array}{l}0.00001 \\
0.00001 \\
0.00001 \\
0.06402 \\
0.00206 \\
0.28160 \\
0.00000 \\
0.63911 \\
0.01319\end{array}$ & $\begin{array}{l}\text { syn } \\
\text { anti } \\
\text { syn } \\
\text { syn } \\
\text { anti } \\
\text { syn } \\
\text { anti } \\
\text { syn } \\
\text { syn }\end{array}$ \\
\hline IA & $\begin{array}{l}\text { REDO_no2phncs_tfs_1 } \\
\text { REDO_no2phncs_tfs_2 } \\
\text { REDO_no2phncs_tfs_3 } \\
\text { REDO_no2phncs_tfs_4 } \\
\text { REDO_no2phncs_tfs_5 } \\
\text { REDO_no2phncs_tfs_6 } \\
\text { REDO_no2phncs_tfs_7 } \\
\text { REDO_no2phncs_tfs_8 } \\
\text { REDO_no2phncs_tfs_9 } \\
\text { REDO_no2phncs_tfs_10 } \\
\text { REDO_s_no2phnco_tfs_1 } \\
\text { REDO_sno2phnco_tfs_2 } \\
\text { REDO_sno2phnco_tfs_3 } \\
\text { REDO_s_no2phnco_tfs_5 } \\
\text { REDO_s_no2phnco_tfs_6 } \\
\text { Weighted }\end{array}$ & $\begin{array}{l}-2084.364560 \\
-2084.380389 \\
-2084.379437 \\
-2084.379430 \\
-2084.368996 \\
-2084.365149 \\
-2084.380479 \\
-2084.365125 \\
-2084.371803 \\
-2084.374637 \\
-2084.374684 \\
-2084.370321 \\
-2084.377090 \\
-2084.375372 \\
-2084.380480\end{array}$ & $\begin{array}{l}-2084.185062 \\
-2084.201624 \\
-2084.199942 \\
-2084.200177 \\
-2084.189192 \\
-2084.186030 \\
-2084.202422 \\
-2084.185769 \\
-2084.192491 \\
-2084.195868 \\
-2084.195527 \\
-2084.193656 \\
-2084.199107 \\
-2084.197673 \\
-2084.202430 \\
-2084.202095\end{array}$ & $\begin{array}{l}0.00000 \\
0.16258 \\
0.02731 \\
0.03505 \\
0.00000 \\
0.00000 \\
0.37892 \\
0.00000 \\
0.00001 \\
0.00036 \\
0.00025 \\
0.00003 \\
0.01126 \\
0.00247 \\
0.38175\end{array}$ & $\begin{array}{l}\text { syn } \\
\text { anti } \\
\text { anti } \\
\text { anti } \\
\text { syn } \\
\text { syn } \\
\text { anti } \\
\text { syn } \\
\text { syn } \\
\text { anti } \\
\text { anti } \\
\text { anti } \\
\text { anti } \\
\text { anti } \\
\text { anti }\end{array}$ \\
\hline \multirow[t]{2}{*}{ TA } & $\begin{array}{l}\text { REDO_1d_nh_tfs_taut_1 } \\
\text { REDO_1d_nh_tfs_taut_1_r1 } \\
\text { Weighted }\end{array}$ & $\begin{array}{l}-2084.379047 \\
-2084.372267\end{array}$ & $\begin{array}{l}-2084.200146 \\
-2084.193559 \\
-2084.200140\end{array}$ & $\begin{array}{l}0.99907 \\
0.00093\end{array}$ & \\
\hline & & $\begin{array}{l}\Delta \mathrm{G}_{\text {calc }}^{\dagger} \\
\Delta_{\mathrm{r}} \mathrm{G}_{\text {calc }}^{0}(\mathrm{IA}) \\
\Delta_{\mathrm{r}} \mathrm{G}_{\text {calc }}^{0}(\mathrm{TA})\end{array}$ & $\begin{array}{l}45.2 \\
-48.4 \\
-43.3\end{array}$ & $\begin{array}{l}\mathrm{kJ} / \mathrm{mol} \\
\mathrm{kJ} / \mathrm{mol} \\
\mathrm{kJ} / \mathrm{mol}\end{array}$ & \\
\hline
\end{tabular}

\begin{tabular}{|c|c|c|c|c|c|}
\hline & Filename & SCF & G298 & Weighting & TS type \\
\hline $2 a$ & REDO_tfs_anion_2 & -1156.953255 & -1156.865502 & & \\
\hline 1e & REDO_1e_1 & -611.067315 & -610.913479 & & \\
\hline TS & $\begin{array}{l}\text { REDO_REDO_REDO_dpc_tfs_1 } \\
\text { REDO_REDO_REDO_dpc_tfs_2 } \\
\text { REDO_REDO_REDO_dpc_tfs_8 } \\
\text { REDO_REDO_REDO_dpc_tfs_9 } \\
\text { REDO_REDO_REDO_dpc_tfs_10 } \\
\text { REDO_dpc_tfs_15_ts1 } \\
\text { REDO_dpc_tfs_15_ts1_r1 } \\
\text { REDO_REDO_REDO_dpc_tfs_16 } \\
\text { REDO_REDO_REDO_dpc_tts_17 } \\
\text { REDO_REDO_REDO_dpc_tfs_22 } \\
\text { dpc_ts_planar_ts1_1_freq } \\
\text { Weighted }\end{array}$ & $\begin{array}{l}-1768.009275 \\
-1768.007034 \\
-1768.009132 \\
-1768.006615 \\
-1768.007881 \\
-1768.016819 \\
-1768.014528 \\
-1768.011578 \\
-1768.011339 \\
-1768.016059 \\
-1768.018808 \\
\end{array}$ & $\begin{array}{l}-1767.744232 \\
-1767.741971 \\
-1767.742792 \\
-1767.741456 \\
-1767.742465 \\
-1767.752810 \\
-1767.750155 \\
-1767.748954 \\
-1767.746130 \\
-1767.752442 \\
-1767.752179 \\
-1767.752456\end{array}$ & $\begin{array}{l}0.00005 \\
0.00000 \\
0.00001 \\
0.00000 \\
0.00001 \\
0.44124 \\
0.02642 \\
0.00740 \\
0.00037 \\
0.29863 \\
0.22587\end{array}$ & \\
\hline IA & $\begin{array}{l}\text { REDO_dpc_tfs_1 } \\
\text { REDO_dpc_tfs_2 } \\
\text { REDO_dpc_tfs_4_2 } \\
\text { REDO_dpc_tfs_5 } \\
\text { REDO_dpc_tfs_7 } \\
\text { REDO_dpc_tfs_8 } \\
\text { REDO_dpc_tfs_9 } \\
\text { REDO_dpc_tfs_10 } \\
\text { REDO_dpc_tfs_15 } \\
\text { REDO_dpc_tfs_16 } \\
\text { REDO_dpc_tfs_17 } \\
\text { REDO_dpc_tfs_19 } \\
\text { REDO_dpc_tfs_22 } \\
\text { REDO_dpc_tfs_24 }\end{array}$ & $\begin{array}{l}-1768.046461 \\
-1768.046410 \\
-1768.046932 \\
-1768.046967 \\
-1768.046810 \\
-1768.048079 \\
-1768.046207 \\
-1768.048246 \\
-1768.048031 \\
-1768.047913 \\
-1768.046768 \\
-1768.048022 \\
-1768.047290 \\
-1768.048770\end{array}$ & $\begin{array}{l}-1767.776754 \\
-1767.777456 \\
-1767.777223 \\
-1767.777321 \\
-1767.777508 \\
-1767.778389 \\
-1767.777254 \\
-1767.779426 \\
-1767.779670 \\
-1767.780166 \\
-1767.777107 \\
-1767.780139 \\
-1767.779486 \\
-1767.780075\end{array}$ & $\begin{array}{l}0.01990 \\
0.01886 \\
0.03278 \\
0.03404 \\
0.02881 \\
0.11060 \\
0.01521 \\
0.13202 \\
0.10513 \\
0.09276 \\
0.02757 \\
0.10414 \\
0.04795 \\
0.23022\end{array}$ & \\
\hline
\end{tabular}




$\begin{array}{lll}\Delta \mathrm{G}_{\text {calc }}^{\ddagger} & 61.7 & \mathrm{~kJ} / \mathrm{mol} \\ \Delta_{\mathrm{r}} \mathrm{G}_{\text {calc }}(\mathrm{IA}) & -8.6 & \mathrm{~kJ} / \mathrm{mol} \\ \Delta_{\mathrm{r}} \mathrm{G}_{\text {calc }}^{0}(\mathrm{TA}) & -35.5 & \mathrm{~kJ} / \mathrm{mol}\end{array}$

\begin{tabular}{|c|c|c|c|c|c|}
\hline \multicolumn{6}{|c|}{ 1f + 2a: DCC + TFS (DMSO) } \\
\hline & Filename & SCF & G298 & Weighting & TS type \\
\hline $2 \mathbf{a}$ & REDO_tfs_anion_2 & -1156.953255 & -1156.865502 & & \\
\hline \multirow[t]{7}{*}{ 1f } & REDO_1f_2 & -618.342039 & -618.048202 & 0.02805 & \\
\hline & REDO_1f_3_low_2 & -618.340326 & -618.048292 & 0.03086 & \\
\hline & REDO_1f_7_low_2 & -618.340334 & -618.048099 & 0.02515 & \\
\hline & REDO_1f_9_low & -618.340265 & -618.047742 & 0.01723 & \\
\hline & REDO $1 \mathrm{f} 11$ & -618.341820 & -618.050185 & 0.22962 & \\
\hline & REDO_1f_-18 & -618.343715 & -618.051194 & 0.66909 & \\
\hline & Weighted ${ }^{-}$ & & -618.050652 & & \\
\hline \multirow[t]{15}{*}{ TS } & REDO_dcc_tfs_1_ts 1 & -1775.268505 & -1774.864956 & 0.00001 & \\
\hline & REDO_dcc_tfs_2_ts 1 & -1775.264741 & -1774.861746 & 0.00000 & \\
\hline & REDO_dcc_tfs_3_ts 1 & -1775.273415 & -1774.870617 & 0.00258 & \\
\hline & REDO $\mathrm{dcc}$ tfs 3 ts 2 & -1775.271707 & -1774.869565 & 0.00084 & \\
\hline & REDO_dcc_tfs-4ts 1 & -1775.266208 & -1774.862460 & 0.00000 & \\
\hline & REDO_dcc_tfs_5_ts 1 & -1775.274648 & -1774.872161 & 0.01324 & \\
\hline & REDO $\mathrm{dcc}$ tfs 6 ts 1 & -1775.270062 & -1774.866779 & 0.00004 & \\
\hline & REDO_dcc_tfs_7_2_ts1 & -1775.267833 & -1774.863197 & 0.00000 & \\
\hline & $\mathrm{REDO}^{-} \mathrm{dcc} \mathrm{tfs}^{-} 8 \mathrm{ts} 1$ & -1775.274801 & -1774.870862 & 0.00334 & \\
\hline & REDO_dcc_tfs_9_ts 1 & -1775.264227 & -1774.859937 & 0.00000 & \\
\hline & REDO_dccttfs 10 ts 1 & -1775.272535 & -1774.869025 & 0.00048 & \\
\hline & REDO_dcc_tfs_-12_ts 1 & -1775.279758 & -1774.875828 & 0.64620 & \\
\hline & REDO_dccttfs_14-ts 1 & -1775.266036 & -1774.860956 & 0.00000 & \\
\hline & dcc_tfs_planar_ts 1 - 2 & -1775.279912 & -1774.875204 & 0.33327 & \\
\hline & Weighted & & -1774.875532 & & \\
\hline \multirow[t]{14}{*}{ IA } & REDO_dcc_tfs_1 & -1775.298155 & -1774.891454 & 0.02693 & \\
\hline & REDO_dcc_tfs_-2 & -1775.299109 & -1774.890780 & 0.01319 & \\
\hline & REDO_dcc_tfs_-3 & -1775.300470 & -1774.893934 & 0.37333 & \\
\hline & REDO_dcc_tfs_-4 & -1775.299251 & -1774.891145 & 0.01941 & \\
\hline & REDO_dcc-tfs-5 & -1775.299914 & -1774.893704 & 0.29266 & \\
\hline & REDO_dcc_tfs_6 & -1775.298011 & -1774.890831 & 0.01391 & \\
\hline & REDO_dcc_tfs_7_2 & -1775.295212 & -1774.887477 & 0.00040 & \\
\hline & $\mathrm{REDO} \mathrm{dcc} \mathrm{tts} 8$ & -1775.294493 & -1774.887314 & 0.00033 & \\
\hline & REDO_dcc_tfs_9 & -1775.296925 & -1774.889454 & 0.00323 & \\
\hline & REDO_dcc-tfs_10 & -1775.298282 & -1774.893574 & 0.25487 & \\
\hline & REDO_dcc_tfs_11 & -1775.296690 & -1774.888606 & 0.00131 & \\
\hline & REDO_dcc_tfs_12_2 & -1775.294434 & -1774.887464 & 0.00039 & \\
\hline & REDO $\mathrm{dcc}$ tfs 14 & -1775.292111 & -1774.884937 & 0.00003 & \\
\hline & Weighted & & -1774.893540 & & \\
\hline \multirow[t]{10}{*}{ TA } & REDO_dcc_tfs_t_1_2 & -1775.329621 & -1774.921375 & 0.11937 & \\
\hline & REDO_dcc $\mathrm{tfs} t-2$ & -1775.328728 & -1774.921107 & 0.08989 & \\
\hline & REDO_dcc_tfs_t_3 & -1775.328754 & -1774.921396 & 0.12206 & \\
\hline & REDO_dcc_tfs_t_5 & -1775.328348 & -1774.921512 & 0.13802 & \\
\hline & REDO $\mathrm{dcc}$ tfs $\mathrm{t} 8$ & -1775.328410 & -1774.922780 & 0.52975 & \\
\hline & REDO_dcc_tfs_t_9 & -1775.325145 & -1774.916771 & 0.00091 & \\
\hline & Weighted & & -1774.922112 & & \\
\hline & & $\Delta \mathrm{G}_{\text {calc }}^{\ddagger}$ & 98.7 & $\mathrm{~kJ} / \mathrm{mol}$ & \\
\hline & & $\Delta_{\mathrm{r}} \mathrm{G}_{\text {calc }}^{0}(\mathrm{IA})$ & 51.5 & $\mathrm{~kJ} / \mathrm{mol}$ & \\
\hline & & $\Delta_{\mathrm{r}} \mathrm{G}_{\text {calc }}^{0}(\mathrm{TA})$ & -23.6 & $\mathrm{~kJ} / \mathrm{mol}$ & \\
\hline
\end{tabular}

\begin{tabular}{|c|c|c|c|c|c|}
\hline & Filename & SCF & G298 & Weighting & TS type \\
\hline $2 \mathbf{a}$ & REDO_tfs_anion_2 & -1156.953255 & -1156.865502 & & \\
\hline $1 \mathrm{~g}$ & REDO_1g_1 & -834.553403 & -834.569592 & & \\
\hline \multirow[t]{4}{*}{ TS } & REDO_cs2_tfs_1_ts1 & -1991.491764 & -1991.402729 & \multirow{4}{*}{$\begin{array}{l}0.00010 \\
0.99936 \\
0.00054\end{array}$} & \\
\hline & REDO_cs2_tfs_2_ts1 & -1991.499701 & -1991.411379 & & \\
\hline & REDO_cs2_tfs_3_ts1 & -1991.494767 & -1991.404282 & & \\
\hline & Weighted & & -1991.411375 & & \\
\hline IA & REDO_cs2_tfs_1 & -1991.531533 & -1991.438800 & 0.05118 & \\
\hline
\end{tabular}




\begin{tabular}{|c|c|c|c|c|c|}
\hline & REDO_cs2_tfs_2 & -1991.533704 & -1991.441545 & 0.93910 & \\
\hline & REDO_cs2_tfs_3 & -1991.529133 & -1991.436254 & 0.00344 & \\
\hline & REDO_cs2_tfs_4 & -1991.530124 & -1991.436822 & 0.00628 & \\
\hline & Weighted & & -1991.441357 & & \\
\hline TA & REDO_cs 2 ttfs_t_1 & -1991.520585 & -1991.431093 & 0.66744 & \\
\hline & REDO_cs2_tfs_t_4 & -1991.520063 & -1991.430435 & 0.33256 & \\
\hline & Weighted & & -1991.430874 & & \\
\hline & & $\begin{array}{l}\Delta \mathrm{G}_{\text {calc }}^{\ddagger} \\
\Delta_{\mathrm{r}} \mathrm{G}_{\text {calc }}^{\text {calc }}(\mathrm{IA}) \\
\Delta_{\mathrm{r}} \mathrm{G}_{\text {calc }}^{0}(\mathrm{TA})\end{array}$ & $\begin{array}{l}54.4 \\
-24.4 \\
3.2\end{array}$ & $\begin{array}{l}\mathrm{kJ} / \mathrm{mol} \\
\mathrm{kJ} / \mathrm{mol} \\
\mathrm{kJ} / \mathrm{mol}\end{array}$ & \\
\hline $1 \mathrm{~h}$ & (DMSO) & & & & \\
\hline & Filename & SCF & G298 & Weighting & TS type \\
\hline $2 a$ & REDO_tfs_anion_2 & -1156.953255 & -1156.865502 & & \\
\hline $1 \mathrm{~h}$ & REDO_1h_1 & -188.649912 & -188.659039 & & \\
\hline TS & REDO_co2_tfs_1_ts1 & -1345.594375 & -1345.499762 & 0.00014 & \\
\hline & REDO_co2_tfs_2_ts1 & -1345.597316 & -1345.501549 & 0.00090 & \\
\hline & REDO_co2_tfs_3_ts1 & -1345.602697 & -1345.508163 & 0.99897 & \\
\hline & Weighted & & -1345.508156 & & \\
\hline IA & REDO_co2_tfs_1 & -1345.618776 & -1345.520473 & 0.09973 & \\
\hline & REDO_co2_tfs_2 & -1345.617719 & -1345.518445 & 0.01162 & \\
\hline & REDO_co2_tfs_-3 & -1345.620356 & -1345.522536 & 0.88865 & \\
\hline & Weighted & & -1345.522282 & & \\
\hline TA & REDO_co2_tfs_t_1 & -1345.605110 & -1345.507343 & 0.51814 & \\
\hline & REDO_co2_tfs_t_3 & -1345.605500 & -1345.507274 & 0.48186 & \\
\hline & Weighted & & -1345.507310 & & \\
\hline & & $\Delta \mathrm{G}_{\text {calc }}^{\ddagger}$ & 35.1 & $\mathrm{~kJ} / \mathrm{mol}$ & \\
\hline & & $\Delta_{\mathrm{r}} \mathrm{G}_{\text {calc }}^{0}(\mathrm{IA})$ & -2.0 & $\mathrm{~kJ} / \mathrm{mol}$ & \\
\hline & & $\Delta_{\mathrm{r}} \mathrm{G}_{\text {calc }}^{0}(\mathrm{TA})$ & 37.3 & $\mathrm{~kJ} / \mathrm{mol}$ & \\
\hline
\end{tabular}


Table S52: Quantum chemically calculated activation and reaction energies for the reactions of heteroallenes 1a-h with nucleophiles $\mathbf{2 n}$ at the IEFPCM(DMSO)/B3LYP-D3/6-311+G(d,p) level of theory.

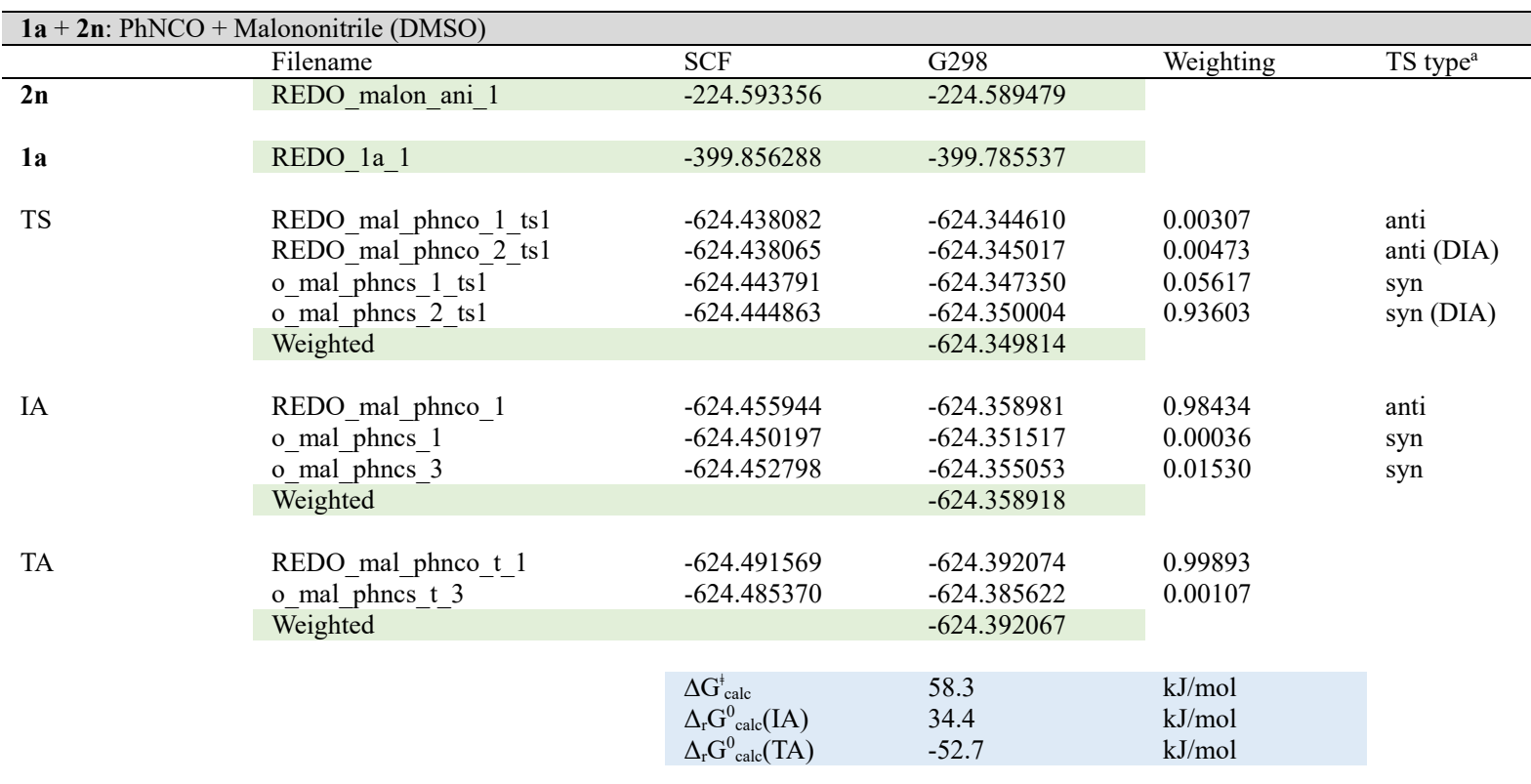

\begin{tabular}{|c|c|c|c|c|c|}
\hline & Filename & SCF & G298 & Weighting & TS type $^{\mathrm{a}}$ \\
\hline $2 n$ & REDO_malon_ani_1 & -224.593356 & -224.589479 & & \\
\hline $1 b$ & REDO_1b_1 & -987.832269 & -987.733337 & & \\
\hline TS & $\begin{array}{l}\text { REDO_mal_tic_1_ts1 } \\
\text { REDO_mal_tic2_ts1 } \\
\text { REDO_mal_tic_3_ts1 } \\
\text { REDO_mal_tic_4_ts1 } \\
\text { REDO_mal_tic_5_ts1 } \\
\text { REDO_mal_s_tic_1_ts } 1 \\
\text { REDO_mals_tic_3_ts1 } \\
\text { REDO_mal_s_tic_4_ts1 } \\
\text { Weighted }\end{array}$ & $\begin{array}{l}-1212.427434 \\
-1212.428343 \\
-1212.427370 \\
-1212.427415 \\
-1212.426843 \\
-1212.426997 \\
-1212.425608 \\
-1212.429301\end{array}$ & $\begin{array}{l}-1212.306913 \\
-1212.305761 \\
-1212.306796 \\
-1212.306891 \\
-1212.305391 \\
-1212.302735 \\
-1212.300983 \\
-1212.304692 \\
-1212.306613\end{array}$ & $\begin{array}{l}0.28868 \\
0.08513 \\
0.25512 \\
0.28217 \\
0.05751 \\
0.00344 \\
0.00054 \\
0.02740\end{array}$ & \\
\hline IA & $\begin{array}{l}\text { REDO_mal_tic_1_2 } \\
\text { REDO_mal_tic_2 } \\
\text { REDO_mal_tic_3 } \\
\text { REDO_mal_tic_4 } \\
\text { REDO_mal_tic_5 } \\
\text { REDO_mal_s_tic_1 } \\
\text { REDO_mal_s_tic_3 } \\
\text { REDO_mal_s_tic_4 } \\
\text { REDO_mal_s_tic_5 } \\
\text { Weighted }\end{array}$ & $\begin{array}{l}-1212.456137 \\
-1212.450984 \\
-1212.456157 \\
-1212.457477 \\
-1212.457397 \\
-1212.451944 \\
-1212.449259 \\
-1212.452709 \\
-1212.451743\end{array}$ & $\begin{array}{l}-1212.330459 \\
-1212.324844 \\
-1212.329974 \\
-1212.330190 \\
-1212.329855 \\
-1212.325204 \\
-1212.321293 \\
-1212.324600 \\
-1212.325358 \\
-1212.330154\end{array}$ & $\begin{array}{l}0.34616 \\
0.00090 \\
0.20684 \\
0.26019 \\
0.18232 \\
0.00132 \\
0.00002 \\
0.00069 \\
0.00155\end{array}$ & \\
\hline \multirow[t]{2}{*}{$\mathrm{TA}$} & $\begin{array}{l}\text { REDO_mal_tic_t_1 } \\
\text { REDO_mal_tic_t_1_r1 } \\
\text { Weighted }\end{array}$ & $\begin{array}{l}-1212.473980 \\
-1212.469505\end{array}$ & $\begin{array}{l}-1212.346147 \\
-1212.341830 \\
-1212.346103\end{array}$ & $\begin{array}{l}0.98982 \\
0.01018\end{array}$ & \\
\hline & & $\begin{array}{l}\Delta \mathrm{G}_{\text {calc }}^{\ddagger} \\
\Delta_{\mathrm{r}} \mathrm{G}_{\text {calc }}^{0}(\mathrm{IA}) \\
\Delta_{\mathrm{r}} \mathrm{G}_{\text {calc }}^{0}(\mathrm{TA})\end{array}$ & $\begin{array}{l}34.6 \\
-27.2 \\
-69.0\end{array}$ & $\begin{array}{l}\mathrm{kJ} / \mathrm{mol} \\
\mathrm{kJ} / \mathrm{mol} \\
\mathrm{kJ} / \mathrm{mol}\end{array}$ & \\
\hline \multicolumn{6}{|c|}{$\mathbf{1 c}+\mathbf{2 n}:$ PhNCS + Malononitrile (DMSO) } \\
\hline $2 n$ & REDO_malon_ani_1 & -224.593356 & -224.589479 & & \\
\hline $1 \mathrm{c}$ & REDO_1c_1 & -722.815578 & -722.749237 & & \\
\hline TS & $\begin{array}{l}\text { REDO_mal_phncs_1_ts1 } \\
\text { REDO_mal_phncs_2_ts } 1 \\
\text { REDO_mal_phncs } 3 \text { ts } 1\end{array}$ & $\begin{array}{l}-947.397521 \\
-947.394127 \\
-947.398446\end{array}$ & $\begin{array}{l}-947.304886 \\
-947.303273 \\
-947.306744\end{array}$ & $\begin{array}{l}0.11695 \\
0.02113 \\
0.83851\end{array}$ & $\begin{array}{l}\text { syn } \\
\text { anti } \\
\text { syn (DIA) }\end{array}$ \\
\hline
\end{tabular}




\begin{tabular}{|c|c|c|c|c|c|}
\hline & REDO_mal_phncs_2_ts1_r2 & -947.394144 & -947.303369 & 0.02342 & anti \\
\hline & Weighted & & -947.306375 & & \\
\hline \multirow[t]{4}{*}{ IA } & REDO_mal_phncs_1 & -947.412810 & -947.316566 & 0.00003 & syn \\
\hline & REDO_mal_phncs_2 & -947.420096 & -947.326279 & 0.99864 & anti \\
\hline & REDO_mal_phncs_3 & -947.415020 & -947.320032 & 0.00133 & syn \\
\hline & Weighted & & -947.326271 & & \\
\hline \multirow[t]{4}{*}{ TA } & REDO_mal_phncs_t_1 & -947.447645 & -947.351187 & 0.92747 & \\
\hline & REDO_mal_phncs_t_3 & -947.445951 & -947.348783 & 0.07253 & \\
\hline & Weighted & & -947.351012 & & \\
\hline & & $\begin{array}{l}\Delta \mathrm{G}_{\text {calc }}^{\ddagger} \\
\Delta_{\mathrm{r}} \mathrm{G}_{\text {calc }}^{0}(\mathrm{IA}) \\
\Delta_{\mathrm{r}} \mathrm{G}_{\text {calc }}^{0}(\mathrm{TA})\end{array}$ & $\begin{array}{l}77.0 \\
24.8 \\
-40.2\end{array}$ & $\begin{array}{l}\mathrm{kJ} / \mathrm{mol} \\
\mathrm{kJ} / \mathrm{mol} \\
\mathrm{kJ} / \mathrm{mol}\end{array}$ & \\
\hline \multicolumn{6}{|c|}{ 1d + 2n: 4-NO ${ }_{2} \mathrm{Ph}-\mathrm{NCS}+$ Malononitrile (DMSO) } \\
\hline & Filename & SCF & G298 & Weighting & TS type $^{\mathrm{a}}$ \\
\hline $2 n$ & REDO_malon_ani_1 & -224.593356 & -224.589479 & & \\
\hline 1d & REDO_1d_1 & -927.385765 & -927.321170 & & \\
\hline \multirow[t]{4}{*}{ TS } & REDO_mal_no2phncs_1_ts1 & -1151.972561 & -1151.882430 & 0.26317 & syn \\
\hline & REDO_mal_no2phncs_2_ts 1 & -1151.968457 & -1151.880722 & 0.04301 & anti \\
\hline & REDO_mal_no2phncs_3_ts1 & -1151.972666 & -1151.883345 & 0.69382 & syn \\
\hline & Weighted & & -1151.882991 & & \\
\hline \multirow[t]{5}{*}{ IA } & REDO_mal_no2phncs_1 & -1151.989926 & -1151.895995 & 0.00003 & syn \\
\hline & REDO_mal_no2phncs_2 & -1151.997490 & -1151.905425 & 0.67810 & anti \\
\hline & REDO_mal_no2phncs_3 & -1151.991269 & -1151.898397 & 0.00039 & syn \\
\hline & s_no2_mal_phnco_2 & -1151.996966 & -1151.904721 & 0.32147 & anti \\
\hline & Weighted & & -1151.905196 & & \\
\hline \multirow[t]{4}{*}{ TA } & REDO_mal_no2phncs_t_1 & -1152.022167 & -1151.928418 & 0.95296 & \\
\hline & REDO_mal_no2phncs_t_3 & -1152.020972 & -1151.925580 & 0.04704 & \\
\hline & Weighted & & -1151.928284 & & \\
\hline & & $\begin{array}{l}\Delta \mathrm{G}_{\text {calc }}^{\ddagger} \\
\Delta_{\mathrm{r}} \mathrm{G}_{\text {calc }}^{0}(\mathrm{IA}) \\
\Delta_{\mathrm{r}} \mathrm{G}_{\text {calc }}^{0}(\mathrm{TA})\end{array}$ & $\begin{array}{l}64.7 \\
6.4 \\
-54.2\end{array}$ & $\begin{array}{l}\mathrm{kJ} / \mathrm{mol} \\
\mathrm{kJ} / \mathrm{mol} \\
\mathrm{kJ} / \mathrm{mol}\end{array}$ & \\
\hline
\end{tabular}

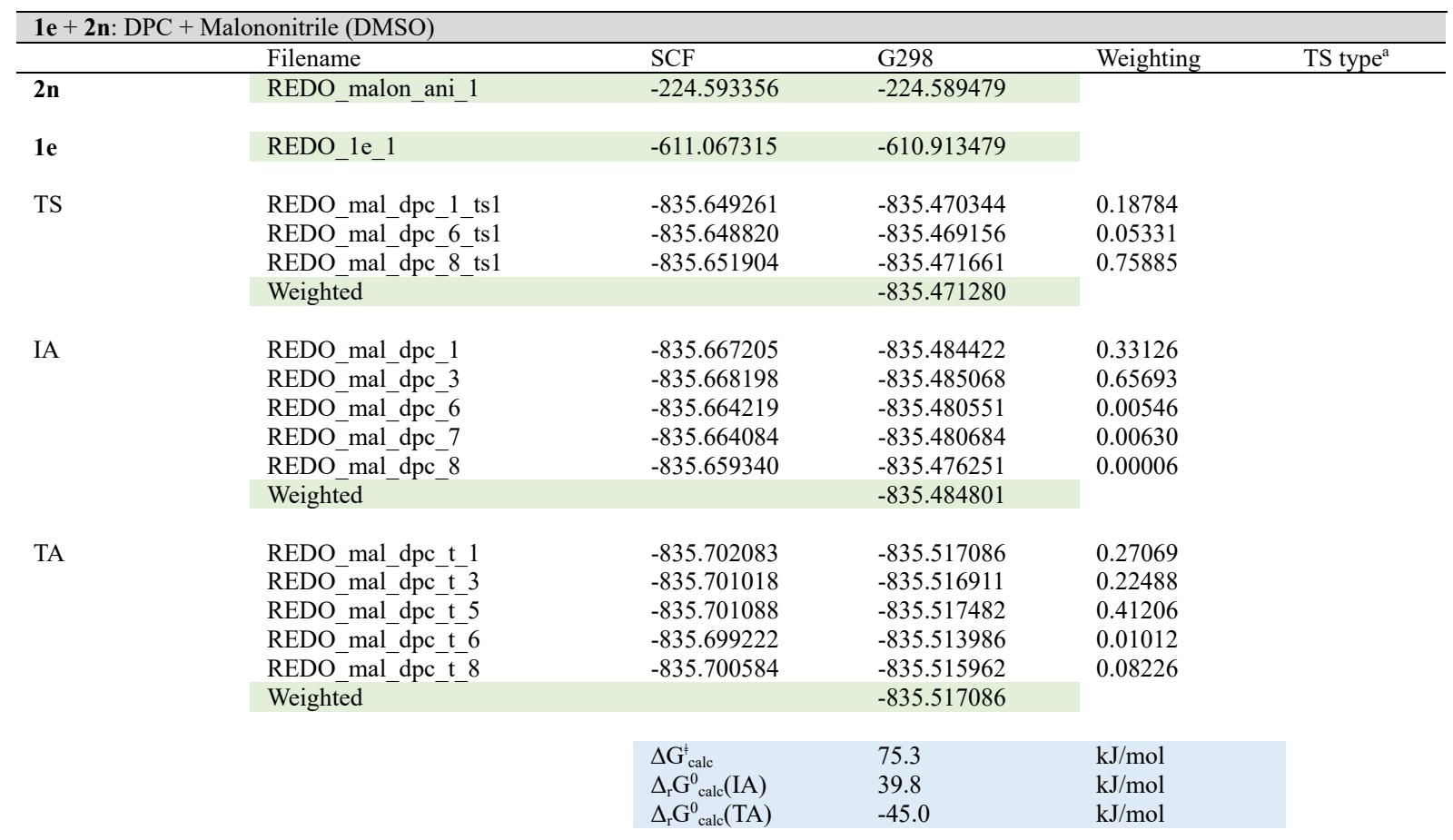

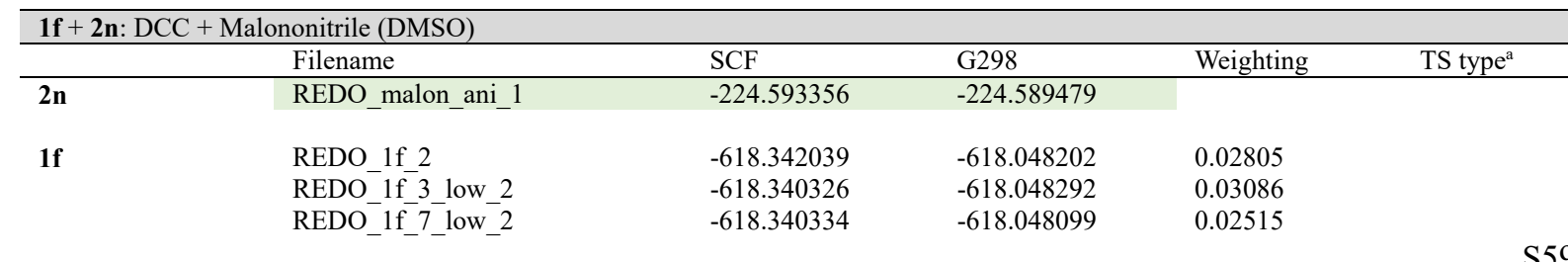




\begin{tabular}{|c|c|c|c|c|c|}
\hline & REDO if 9 low & -618.340265 & -618.047742 & 0.01723 & \\
\hline & REDO_1f_- 11 & -618.341820 & -618.050185 & 0.22962 & \\
\hline & REDO_1f_18 & -618.343715 & -618.051194 & 0.66909 & \\
\hline & Weighted & & -618.050652 & & \\
\hline \multirow[t]{5}{*}{ TS } & REDO_mal_dcc_1_ts1 & -842.905654 & -842.588933 & 0.00636 & \\
\hline & REDO_mal_dcc_3_ts 1 & -842.904320 & -842.586511 & 0.00049 & \\
\hline & REDO mal dec 4 ts 1 & -842.904462 & -842.586433 & 0.00045 & \\
\hline & mal_dec_plan_opt_ts1_2 & -842.913206 & -842.593697 & 0.99271 & \\
\hline & Weighted & & -842.593660 & & \\
\hline \multirow[t]{3}{*}{ IA } & REDO_mal_dcc_1 & -842.921952 & -842.601942 & 0.97230 & \\
\hline & REDO mal dec 3 & -842.919112 & -842.598586 & 0.02770 & \\
\hline & Weighted & & -842.601849 & & \\
\hline \multirow[t]{9}{*}{ TA } & REDO_mal_dcc_t_1 & -842.968946 & -842.647067 & 0.40032 & \\
\hline & $\mathrm{REDO}^{-} \mathrm{mal} \mathrm{dcc}^{-} \mathrm{t} 3$ & -842.969307 & -842.647076 & 0.40410 & \\
\hline & REDO mal dec t 4 & -842.967077 & -842.643493 & 0.00905 & \\
\hline & REDO_mal_dcctt 5 & -842.968317 & -842.644888 & 0.03970 & \\
\hline & REDO mal dect 8 & -842.964524 & -842.641661 & 0.00130 & \\
\hline & REDO_mal_dec_t_9 & -842.964575 & -842.642321 & 0.00261 & \\
\hline & REDO mal dec $\mathrm{t} 10$ & -842.968920 & -842.646096 & 0.14292 & \\
\hline & Weighted & & -842.646794 & & \\
\hline & & $\begin{array}{l}\Delta \mathrm{G}_{\text {calc }}^{\ddagger} \\
\Delta_{\mathrm{r}} \mathrm{G}_{\text {calc }}(\mathrm{IA}) \\
\Delta_{\mathrm{r}} \mathrm{G}_{\text {calc }}^{0}(\mathrm{TA})\end{array}$ & $\begin{array}{l}114.1 \\
92.6 \\
-25.4\end{array}$ & $\begin{array}{l}\mathrm{kJ} / \mathrm{mol} \\
\mathrm{kJ} / \mathrm{mol} \\
\mathrm{kJ} / \mathrm{mol}\end{array}$ & \\
\hline \multicolumn{6}{|c|}{$1 \mathrm{~g}+\mathbf{2 n}: \mathrm{CS}_{2}+$ Malononitrile (DMSO) } \\
\hline & Filename & SCF & G298 & Weighting & TS type $\mathrm{a}^{\mathrm{a}}$ \\
\hline $2 n$ & REDO_malon_ani_1 & -224.593356 & -224.589479 & & \\
\hline $1 \mathrm{~g}$ & REDO_1g_1 & -834.553403 & -834.569592 & & \\
\hline \multirow[t]{2}{*}{ TS } & REDO_mal_cs2_1_ts1 & -1059.133694 & -1059.129582 & 1.00000 & (DIA) \\
\hline & Weighted & & -1059.129582 & & \\
\hline \multirow[t]{2}{*}{ IA } & REDO_mal_cs2_1 & -1059.154203 & -1059.148106 & 1.00000 & \\
\hline & Weighted & & -1059.148106 & & \\
\hline \multirow[t]{6}{*}{$\mathrm{TA}$} & REDO_mal_cs2_t_1 & -1059.167357 & -1059.162146 & 0.50751 & \\
\hline & REDO $\mathrm{mal}_{\mathrm{cs} 2 \mathrm{t} 2}$ & -1059.167286 & -1059.162117 & 0.49249 & \\
\hline & Weighted & & -1059.162132 & & \\
\hline & & $\Delta \mathrm{G}_{\text {calc }}^{\ddagger}$ & 69.5 & $\mathrm{~kJ} / \mathrm{mol}$ & \\
\hline & & $\Delta_{\mathrm{r}} \mathrm{G}_{\text {calc }}^{0}(\mathrm{IA})$ & 20.9 & $\mathrm{~kJ} / \mathrm{mol}$ & \\
\hline & & $\Delta_{\mathrm{r}} \mathrm{G}_{\text {calc }}^{0}(\mathrm{TA})$ & -15.9 & $\mathrm{~kJ} / \mathrm{mol}$ & \\
\hline \multicolumn{6}{|c|}{$\mathbf{1 h}+\mathbf{2 n}: \mathrm{CO}_{2}+$ Malononitrile (DMSO) } \\
\hline & Filename & SCF & G298 & Weighting & TS type ${ }^{a}$ \\
\hline $2 n$ & REDO_malon_ani_1 & -224.593356 & -224.589479 & & \\
\hline $1 \mathrm{~h}$ & REDO_1h_1 & -188.649912 & -188.659039 & & \\
\hline \multirow[t]{2}{*}{ TS } & REDO_mal_co2_1_ts1 & -413.237820 & -413.227680 & 1.00000 & (DIA) \\
\hline & Weighted & & -413.227680 & & \\
\hline \multirow[t]{2}{*}{ IA } & REDO_mal_co2_1 & -413.244916 & -413.232054 & 1.00000 & \\
\hline & Weighted & & -413.232054 & & \\
\hline \multirow[t]{6}{*}{$\mathrm{TA}$} & REDO_mal_co2_t_1 & -413.251848 & -413.237199 & 0.03027 & \\
\hline & REDO_mal_co2_t_2 & -413.255476 & -413.240469 & 0.96973 & \\
\hline & Weighted & & -413.240370 & & \\
\hline & & $\Delta \mathrm{G}_{\mathrm{calc}}^{\ddagger}$ & 46.8 & $\mathrm{~kJ} / \mathrm{mol}$ & \\
\hline & & $\Delta_{\mathrm{r}} \mathrm{G}_{\text {calc }}^{0}(\mathrm{IA})$ & 35.3 & $\mathrm{~kJ} / \mathrm{mol}$ & \\
\hline & & $\Delta_{\mathrm{r}} \mathrm{G}_{\text {calc }}^{0}(\mathrm{TA})$ & 13.5 & $\mathrm{~kJ} / \mathrm{mol}$ & \\
\hline
\end{tabular}

${ }^{a}$ DIA indicates those files that were used for the distortion interaction analysis. 
Table S53: Quantum chemically calculated activation and reaction energies for the reactions of heteroallenes 1a-h with nucleophiles $\mathbf{2 j}$ at the IEFPCM(DMSO)/B3LYP-D3/6-311+G(d,p) level of theory.

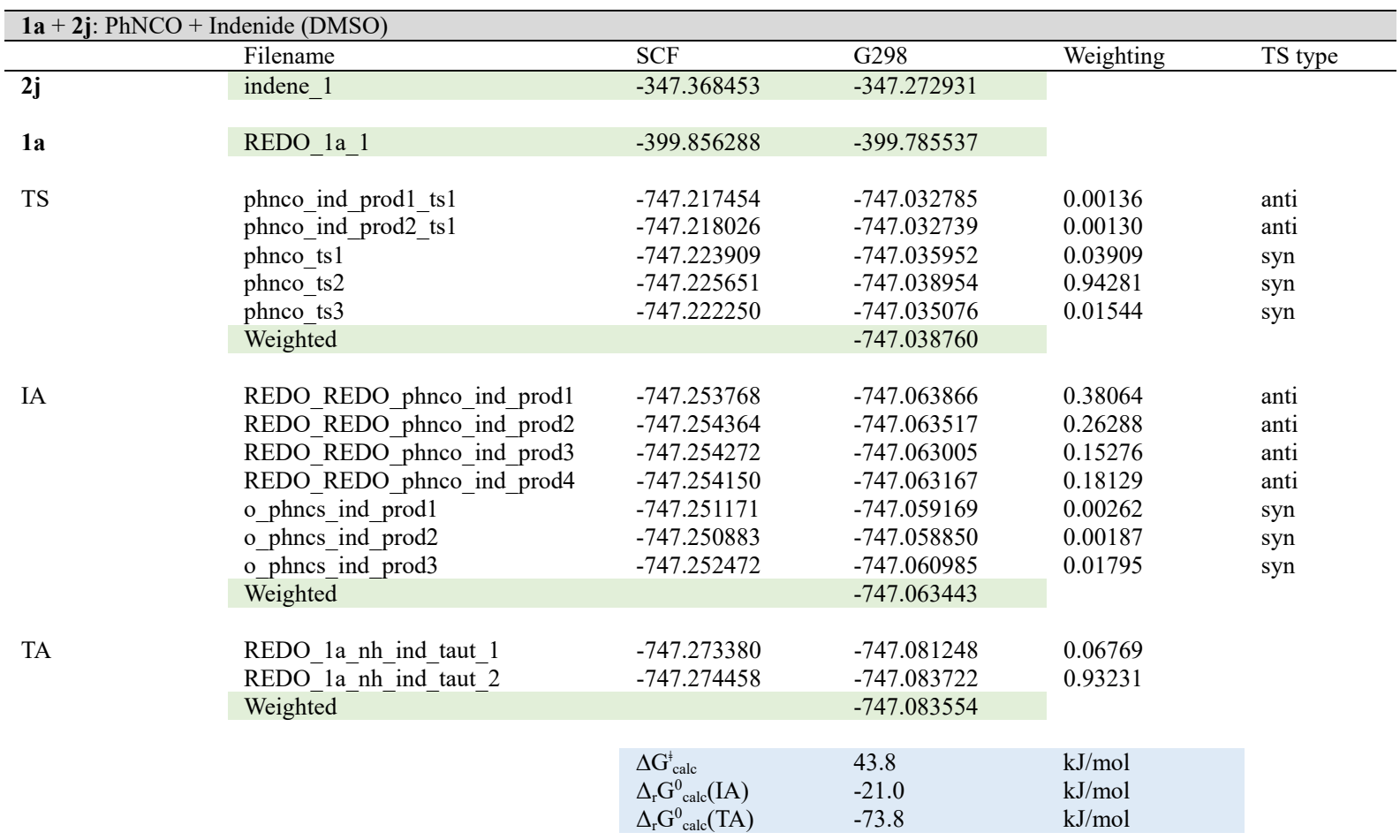

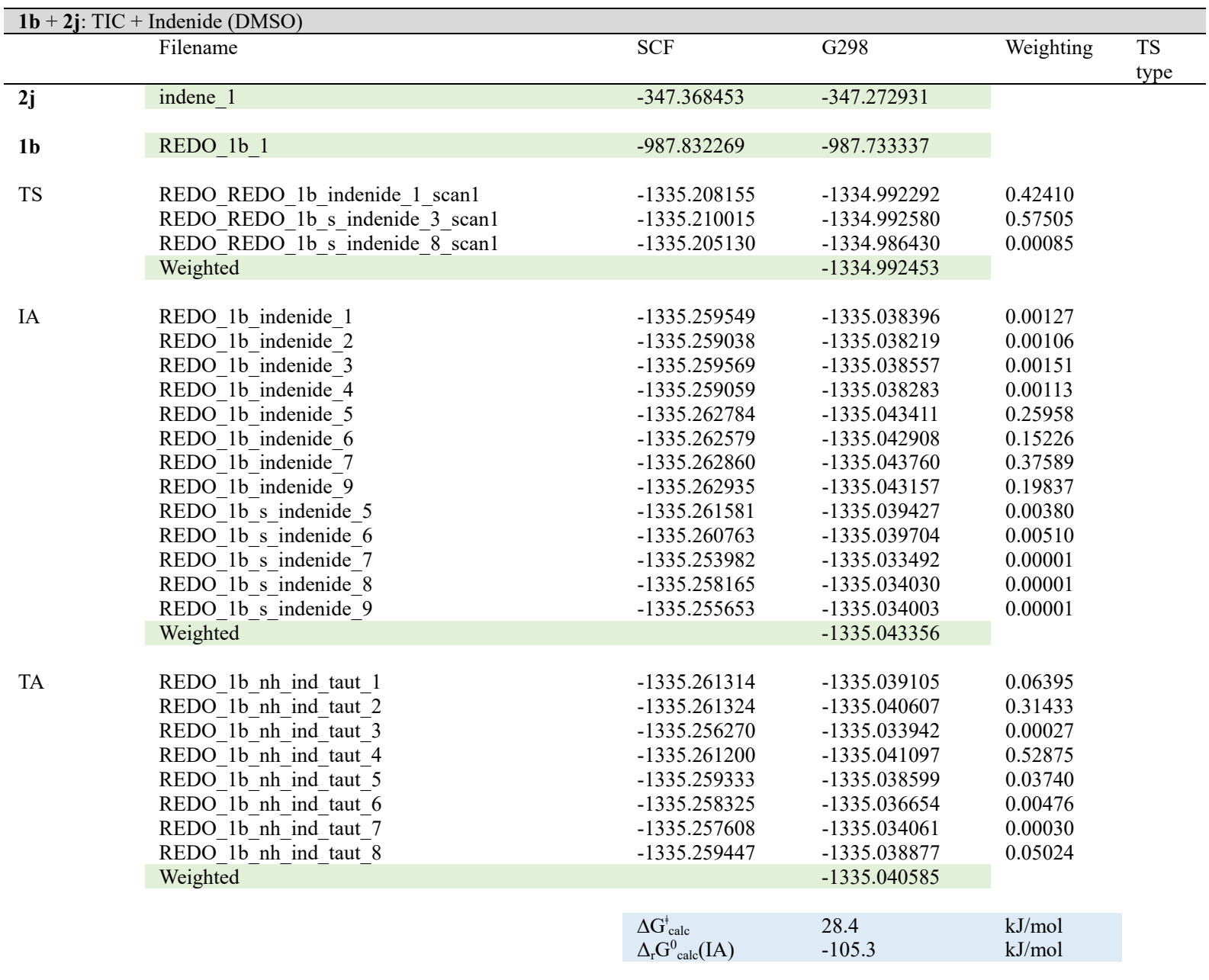


$\begin{array}{lll}\Delta_{\mathrm{r}} \mathrm{G}_{\text {calc }}^{0}(\mathrm{TA}) & -98.0 & \mathrm{~kJ} / \mathrm{mol}\end{array}$

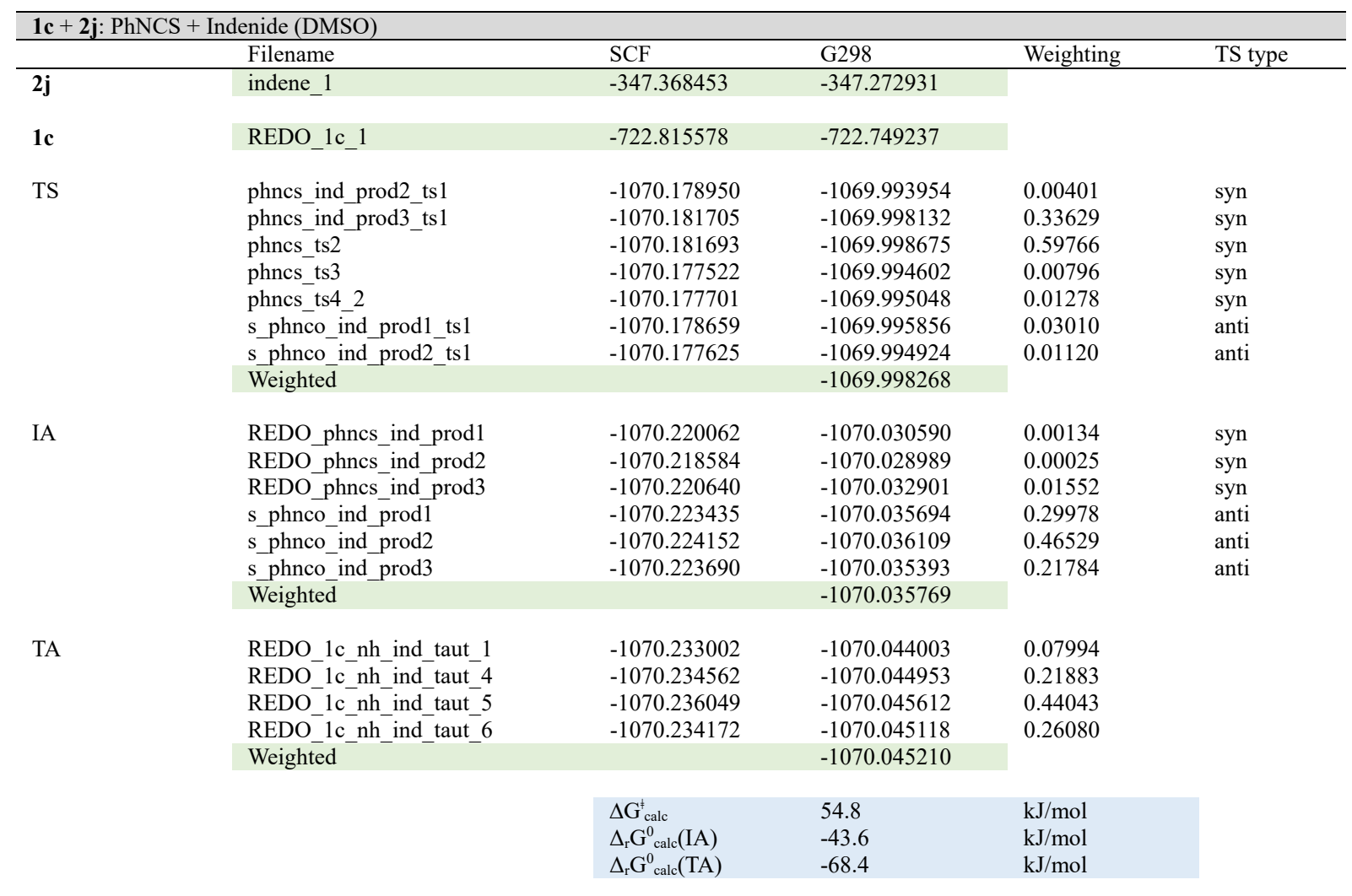

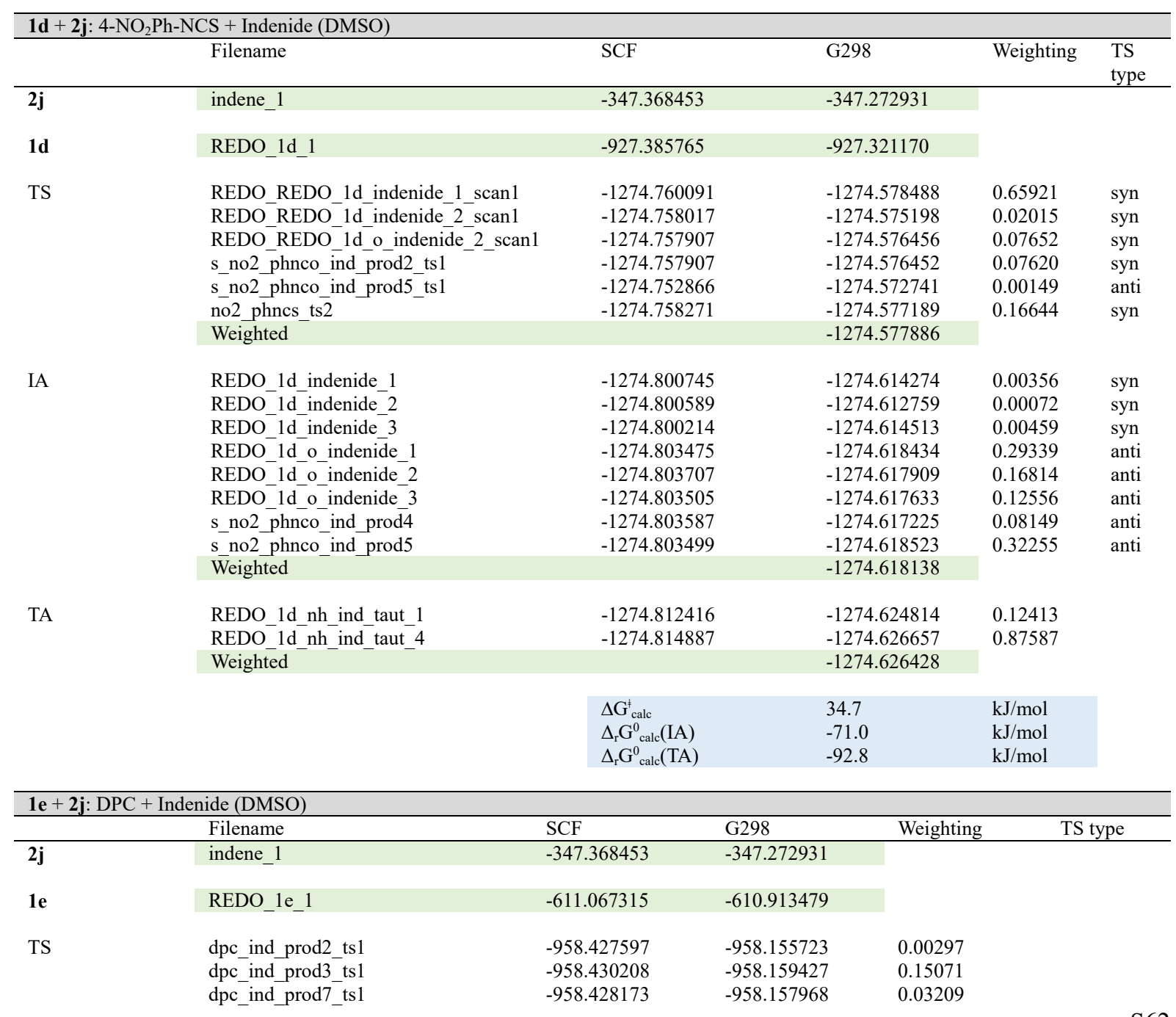


IA

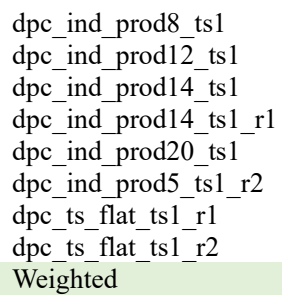

REDO REDO dpc ind prod2

REDO_REDO_dpc_ind_prod3

REDO REDO dpc ind prod5

REDO_REDO_dpc_ind_prod6

REDO_REDO_dpc_ind_prod7

REDO REDO dpc ind prod8

REDO_REDO_dpc_ind_prod 11

REDO REDO dpc ind prod 13

REDO REDO dpc ind prod 14

REDO_REDO_dpc_ind_prod17_2

REDO REDO dpc ind prod 20

Weighted

TA

REDO_dpc_indenide_t_1
REDO_dpc_indenide_t_3_2

REDO dpc indenide t 4

REDO_dpc_indenide_t_5

REDO $\mathrm{dpc}^{-}$indenide $\mathrm{t}$ ?

REDO $d p c$ indenide $t 9$

REDO_dpc_indenide_t 10

Weighted
$-958.429855$

$-958.427376$

$-958.431899$

$-958.430792$

$-958.430408$

$-958.427625$

$-958.430792$

$-958.432045$

$\begin{array}{ll}-958.159413 & 0.14852 \\ -958.158617 & 0.06387 \\ -958.160154 & 0.32558 \\ -958.157211 & 0.01437 \\ -958.158231 & 0.04240 \\ -958.155275 & 0.00185 \\ -958.158827 & 0.07974 \\ -958.159343 & 0.13791 \\ -958.159402 & \\ & \\ -958.189932 & 0.02521 \\ -958.191401 & 0.11963 \\ -958.190909 & 0.07099 \\ -958.191080 & 0.08516 \\ -958.192097 & 0.25034 \\ -958.191100 & 0.08696 \\ -958.191704 & 0.16494 \\ -958.191474 & 0.12926 \\ -958.187297 & 0.00154 \\ -958.190837 & 0.06580 \\ -958.185199 & 0.00017 \\ -958.191465 & \\ & \\ -958.209394 & 0.07895 \\ -958.211440 & 0.69024 \\ -958.209360 & 0.07609 \\ -958.209392 & 0.07875 \\ -958.208699 & 0.03776 \\ -958.207581 & 0.01155 \\ -958.208371 & 0.02666 \\ -958.210729 & \\ 63.0 & \mathrm{~kJ} / \mathrm{mol} \\ -21.2 & \mathrm{~kJ} / \mathrm{mol} \\ -71.8 & \mathrm{~kJ} / \mathrm{mol}\end{array}$

$\begin{array}{lll}-958.466264 & -958.189932 & 0.02521\end{array}$

$\begin{array}{lll}-958.467179 & -958.191401 & 0.11963\end{array}$

$\begin{array}{lll}-958.466937 & -958.190909 & 0.07099\end{array}$

$\begin{array}{lll}-958.466493 & -958.191080 & 0.08516\end{array}$

$\begin{array}{lll}-958.468238 & -958.192097 & 0.25034\end{array}$

$\begin{array}{lll}-958.466544 & -958.191100 & 0.08696\end{array}$

$\begin{array}{lll}-958.467623 & -958.191704 & 0.16494\end{array}$

$\begin{array}{lll}-958.467490 & -958.191474 & 0.12926\end{array}$

$\begin{array}{lll}-958.463577 & -958.187297 & 0.00154 \\ -958.466430 & -958.190837 & 0.06580\end{array}$

$-958.461994$

$-958.486460$

$-958.486443$

$-958.486457$

$-958.486678$

$-958.486099$

$-958.485308$

$-958.485712$

$\mathrm{kJ} / \mathrm{mol}$

\begin{tabular}{|c|c|c|c|c|c|}
\hline \multicolumn{6}{|c|}{$\mathbf{1 f}+\mathbf{2 j}$ : DCC + Indenide (DMSO) } \\
\hline & Filename & SCF & G298 & Weighting & TS type \\
\hline $2 \mathbf{j}$ & indene_1 & -347.368453 & -347.272931 & & \\
\hline \multirow[t]{7}{*}{$1 \mathrm{f}$} & REDO 1f 2 & -618.342039 & -618.048202 & 0.02805 & \\
\hline & REDO $1 \mathrm{f} 3$ low 2 & -618.340326 & -618.048292 & 0.03086 & \\
\hline & REDO_1f_7_low_2 & -618.340334 & -618.048099 & 0.02515 & \\
\hline & REDO $1 \mathrm{f} 9$ low & -618.340265 & -618.047742 & 0.01723 & \\
\hline & REDO_1f_-11 & -618.341820 & -618.050185 & 0.22962 & \\
\hline & REDO $1 \mathrm{f} 18$ & -618.343715 & -618.051194 & 0.66909 & \\
\hline & Weighted & & -618.050652 & & \\
\hline \multirow[t]{11}{*}{ TS } & REDO dec ind 1 ts 1 & -965.684855 & -965.275035 & 0.00001 & \\
\hline & REDO_dcc_ind_2_ts1 & -965.692623 & -965.282467 & 0.03445 & \\
\hline & REDO $\mathrm{dcc}$ ind 3 ts 1 & -965.693615 & -965.284978 & 0.49352 & \\
\hline & REDO_dcc_ind_3_ts1_r1 & -965.692571 & -965.282476 & 0.03478 & \\
\hline & $\mathrm{REDO}^{-} \mathrm{dcc}^{-}$ind 4 ts $1^{-}$ & -965.686127 & -965.276456 & 0.00006 & \\
\hline & REDO_dce_ind 5 ts 1 & -965.692623 & -965.282465 & 0.03438 & \\
\hline & REDO_dce ind 6 ts 1 & -965.693331 & -965.284424 & 0.27419 & \\
\hline & dec ind flat ts 1 r1 & -965.691454 & -965.280598 & 0.00475 & \\
\hline & dcc_ind_flat_ts_1_r2 & -965.691911 & -965.281305 & 0.01005 & \\
\hline & $\mathrm{dcc}$ ind flat ts $1 \mathrm{r} 3$ & -965.693558 & -965.283594 & 0.11380 & \\
\hline & Weightē & & -965.284350 & & \\
\hline \multirow[t]{7}{*}{ IA } & REDO dec ind 1 & -965.713420 & -965.299899 & 0.00063 & \\
\hline & REDO_dcc_ind_2 & -965.717595 & -965.304640 & 0.09658 & \\
\hline & REDO_dcc_ind_3 & -965.718980 & -965.306189 & 0.49920 & \\
\hline & REDO_dcc_ind-4 & -965.713242 & -965.300080 & 0.00077 & \\
\hline & REDO_dcc_ind_5 & -965.717829 & -965.305015 & 0.14372 & \\
\hline & REDO_dcc_ind_6 & -965.719029 & -965.305571 & 0.25909 & \\
\hline & Weighted & & -965.305702 & & \\
\hline \multirow[t]{9}{*}{ TA } & REDO_dcc_ind_t_1 & -965.750628 & -965.335900 & 0.17953 & \\
\hline & REDO_dcc_ind_t_2 & -965.750560 & -965.336091 & 0.21984 & \\
\hline & REDO_dcc_ind_t_3 & -965.750307 & -965.335807 & 0.16276 & \\
\hline & REDO_dcc_ind_t_4 & -965.750169 & -965.336659 & 0.40167 & \\
\hline & REDO $\mathrm{dcc}$ ind $\mathrm{t} 5$ & -965.747984 & -965.333593 & 0.01557 & \\
\hline & REDO_dcc_ind_t_6 & -965.748189 & -965.333401 & 0.01270 & \\
\hline & REDO $\mathrm{dcc}^{-}$ind $\mathrm{t} 7$ & -965.747530 & -965.332803 & 0.00673 & \\
\hline & REDO_dcc_ind_t_9 & -965.745927 & -965.331185 & 0.00121 & \\
\hline & Weighted & & -965.336138 & & \\
\hline
\end{tabular}




\begin{tabular}{|c|c|c|c|c|c|}
\hline & & $\begin{array}{l}\Delta \mathrm{G}_{\text {calc }}^{\ddagger} \\
\Delta_{\mathrm{r}} \mathrm{G}_{\text {calc }}^{0}(\mathrm{IA}) \\
\Delta_{\mathrm{r}} \mathrm{G}_{\text {calc }}^{0}(\mathrm{TA})\end{array}$ & $\begin{array}{l}95.1 \\
39.0 \\
-40.9\end{array}$ & $\begin{array}{l}\mathrm{kJ} / \mathrm{mol} \\
\mathrm{kJ} / \mathrm{mol} \\
\mathrm{kJ} / \mathrm{mol}\end{array}$ & \\
\hline \multicolumn{6}{|c|}{$\mathbf{1 g}+\mathbf{2 j}: \mathrm{CS}_{2}+$ Indenide (DMSO) } \\
\hline & Filename & SCF & G298 & Weighting & TS type \\
\hline $2 \mathbf{j}$ & indene_1 & -347.368453 & -347.272931 & & \\
\hline $1 g$ & REDO_1g_1 & -834.553403 & -834.569592 & & \\
\hline \multirow[t]{2}{*}{ TS } & scan1_ts1 & -1181.921046 & -1181.824824 & \multirow[t]{2}{*}{1.00000} & \\
\hline & Weighted & & -1181.824824 & & \\
\hline \multirow[t]{2}{*}{ IA } & product_1 & -1181.961863 & -1181.860997 & \multirow[t]{2}{*}{1.00000} & \\
\hline & Weighted & & -1181.860997 & & \\
\hline \multirow[t]{5}{*}{ TA } & REDO cs 2 indenide $t 12$ & -1181.958416 & -1181.861463 & \multirow{4}{*}{$\begin{array}{l}0.28681 \\
0.00590 \\
0.70729\end{array}$} & \\
\hline & REDO_cs 2 - indenide_t_2 & -1181.956082 & -1181.857800 & & \\
\hline & $\mathrm{s}$ cco 2 indenide_t_3 & -1181.959641 & -1181.862315 & & \\
\hline & Weighted & & -1181.862044 & & \\
\hline & & $\begin{array}{l}\Delta \mathrm{G}_{\text {calc }}^{\ddagger} \\
\Delta_{\mathrm{r}} \mathrm{G}_{\text {calc }}^{0}(\mathrm{IA}) \\
\Delta_{\mathrm{r}} \mathrm{G}_{\text {calc }}^{0}(\mathrm{TA})\end{array}$ & $\begin{array}{l}38.6 \\
-56.4 \\
-59.2\end{array}$ & $\begin{array}{l}\mathrm{kJ} / \mathrm{mol} \\
\mathrm{kJ} / \mathrm{mol} \\
\mathrm{kJ} / \mathrm{mol}\end{array}$ & \\
\hline \multicolumn{6}{|c|}{$\mathbf{1 h}+\mathbf{2} \mathbf{j}: \mathrm{CO}_{2}+$ Indenide (DMSO) } \\
\hline & Filename & SCF & G298 & Weighting & TS type \\
\hline $2 \mathbf{j}$ & indene_1 & -347.368453 & -347.272931 & & \\
\hline $1 \mathrm{~h}$ & REDO_1h_1 & -188.649912 & -188.659039 & & \\
\hline \multirow[t]{3}{*}{ TS } & $\mathrm{co} 2$ ts 2 & -536.018073 & -535.916196 & \multirow{3}{*}{$\begin{array}{l}0.44922 \\
0.55078\end{array}$} & \\
\hline & scan2_ts 1 & -536.017850 & -535.916388 & & \\
\hline & Weighted & & -535.916302 & & \\
\hline \multirow[t]{3}{*}{ IA } & product_1 & -536.042354 & -535.936275 & \multirow{3}{*}{$\begin{array}{l}0.51767 \\
0.48233\end{array}$} & \\
\hline & product_co2_rot2 & -536.042260 & -535.936208 & & \\
\hline & Weighted & & -535.936243 & & \\
\hline \multirow[t]{4}{*}{ TA } & REDO_co2_indenide_t_2 & -536.036140 & -535.929659 & \multirow{3}{*}{$\begin{array}{l}0.01049 \\
0.98951\end{array}$} & \\
\hline & REDO_co2_indenide_t_3 & -536.040902 & -535.933948 & & \\
\hline & Weighted & & -535.933903 & & \\
\hline & & $\begin{array}{l}\Delta \mathrm{G}_{\text {calc }}^{\ddagger} \\
\Delta_{\mathrm{r}} \mathrm{G}_{\text {calc }}^{0}(\mathrm{IA}) \\
\Delta_{\mathrm{r}} \mathrm{G}_{\text {calc }}^{0}(\mathrm{TA})\end{array}$ & $\begin{array}{l}33.2 \\
-19.1 \\
-13.0 \\
\end{array}$ & $\begin{array}{l}\mathrm{kJ} / \mathrm{mol} \\
\mathrm{kJ} / \mathrm{mol} \\
\mathrm{kJ} / \mathrm{mol}\end{array}$ & \\
\hline
\end{tabular}

Table S54: Summary of Quantum Chemically Calculated Gibbs Activation and Reaction Energies $\left(\mathrm{kJ} \mathrm{mol}^{-1}\right)$ for Reactions of Heteroallenes $\mathbf{1}$ with the Carbanions $\mathbf{2 a}, \mathbf{2 n}$, and $\mathbf{2 j}$ (in DMSO).

\begin{tabular}{l|l|lll|lll|lll}
\hline $\mathbf{1}$ & $E$ & \multicolumn{3}{|c|}{$\mathbf{2 a}$} & \multicolumn{3}{c|}{$\mathbf{2 n}$} & \multicolumn{3}{c}{$\mathbf{2 j}$} \\
\hline & & $\Delta G^{\ddagger}{ }_{\text {calc }}$ & $\begin{array}{l}\Delta_{\mathrm{r}} \mathrm{G}^{0} \\
\text { calc }(\mathrm{IA})\end{array}$ & $\begin{array}{l}\Delta_{\mathrm{r}} \mathrm{G}^{0} \\
\text { calc(TA) }\end{array}$ & $\Delta G^{\ddagger}$ calc & $\begin{array}{l}\Delta_{\mathrm{r}} \mathrm{G}^{0} \\
\text { calc(IA) }\end{array}$ & $\begin{array}{l}\Delta_{\mathrm{r}} \mathrm{G}^{0} \\
\text { calc(TA) }\end{array}$ & $\Delta G^{\ddagger}$ calcd & $\begin{array}{l}\Delta_{\mathrm{r}} \mathrm{G}^{0} \\
\text { calc (IA) }\end{array}$ & $\begin{array}{l}\Delta_{\mathrm{r}} \mathrm{G}^{0} \\
\text { calc(TA) }\end{array}$ \\
\hline $\mathbf{1 a}$ & -15.38 & 40.6 & -6.7 & -36.1 & 58.3 & 34.4 & -52.7 & 43.8 & -21.0 & -73.8 \\
$\mathbf{1 b}$ & -7.69 & 24.9 & -77.8 & -62.8 & 34.6 & -27.2 & -69.0 & 28.4 & -105.3 & -98.0 \\
$\mathbf{1 c}$ & -18.15 & 60.8 & -25.9 & -26.7 & 77.0 & 24.8 & -40.2 & 54.8 & -43.6 & -68.4 \\
$\mathbf{1 d}$ & -15.89 & 45.2 & -48.4 & -43.3 & 64.7 & 6.4 & -54.2 & 34.7 & -71.0 & -92.8 \\
$\mathbf{1 e}$ & -20.14 & 61.7 & -8.6 & -35.5 & 75.3 & 39.8 & -45.0 & 63.0 & -21.2 & -71.8 \\
$\mathbf{1 f}$ & - & 98.7 & 51.5 & -23.6 & 114.1 & 92.6 & -25.4 & 95.1 & 39.0 & -40.9 \\
$\mathbf{1 g}$ & -17.70 & 54.4 & -24.4 & 3.2 & 69.5 & 20.9 & -15.9 & 38.6 & -56.4 & -59.2 \\
$\mathbf{1 h}$ & $(-16.3)$ & 35.1 & -2.0 & 37.3 & 46.8 & 35.3 & 13.5 & 33.2 & -19.1 & -13.0 \\
\hline
\end{tabular}




\section{Correlations involved Quantum Chemically Calculated Activation and Reaction Gibbs Energies}

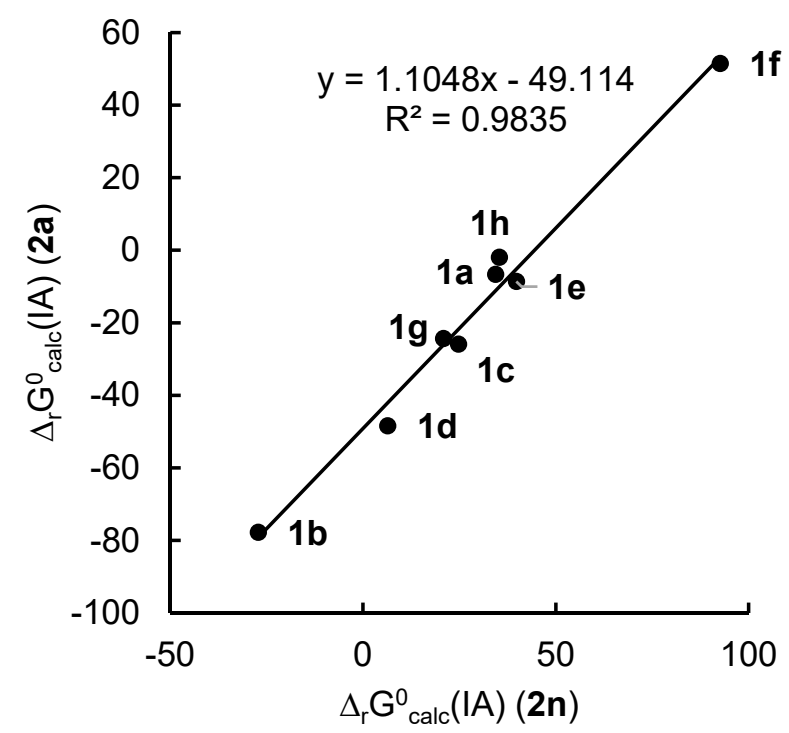

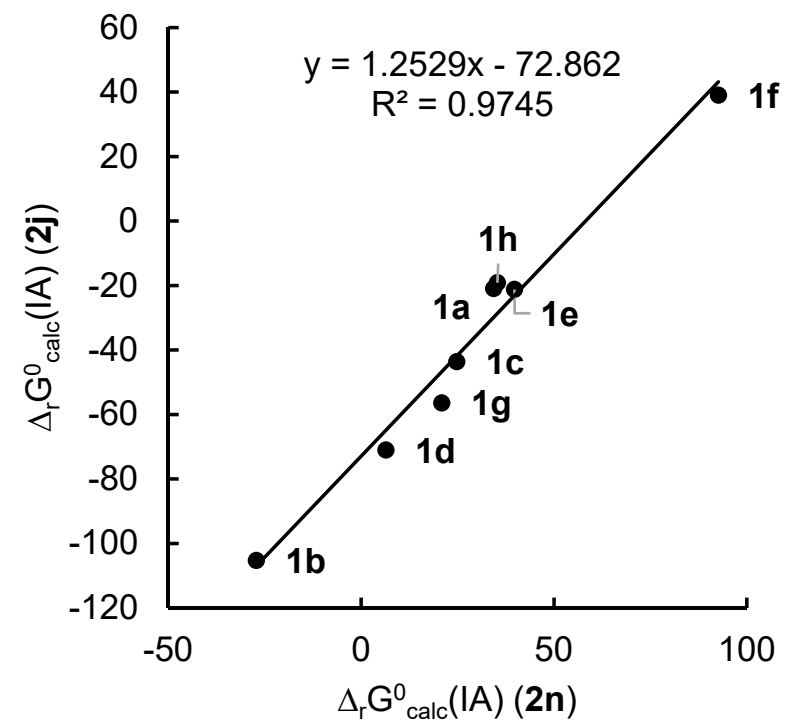

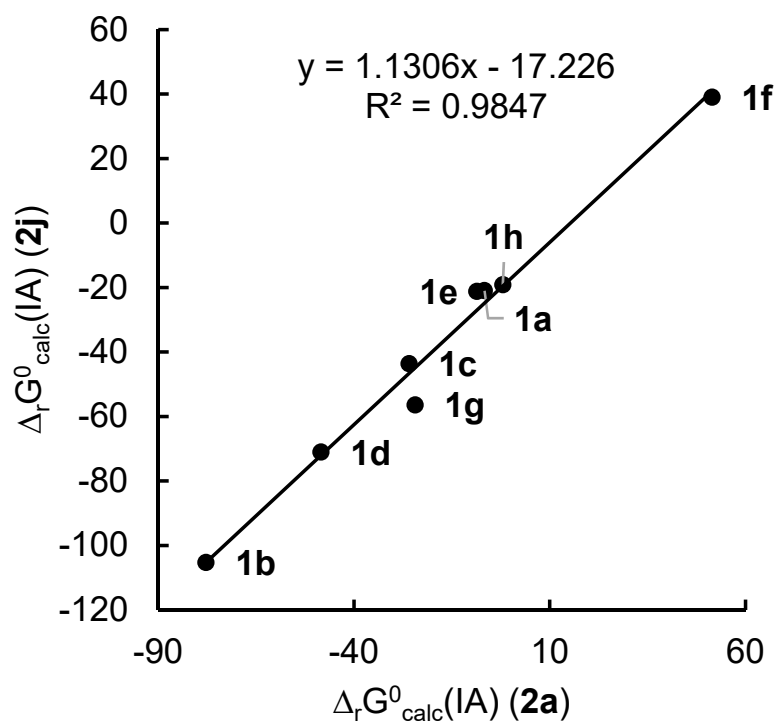

Figure S23. Correlation between Gibbs reaction energies $\Delta_{\mathrm{r}} \mathrm{G}^{0}$ calc $(\mathbf{I A})\left(\mathrm{kJ} \mathrm{mol}^{-1}\right)$ for the reactions of heteroallenes $\mathbf{1}$ with different carbanions $\mathbf{2}(\mathbf{2 a}, \mathbf{n}, \mathbf{j})$ calculated at the IEFPCM(DMSO)/B3LYP-D3/6-311+G(d,p) level of theory. 
A

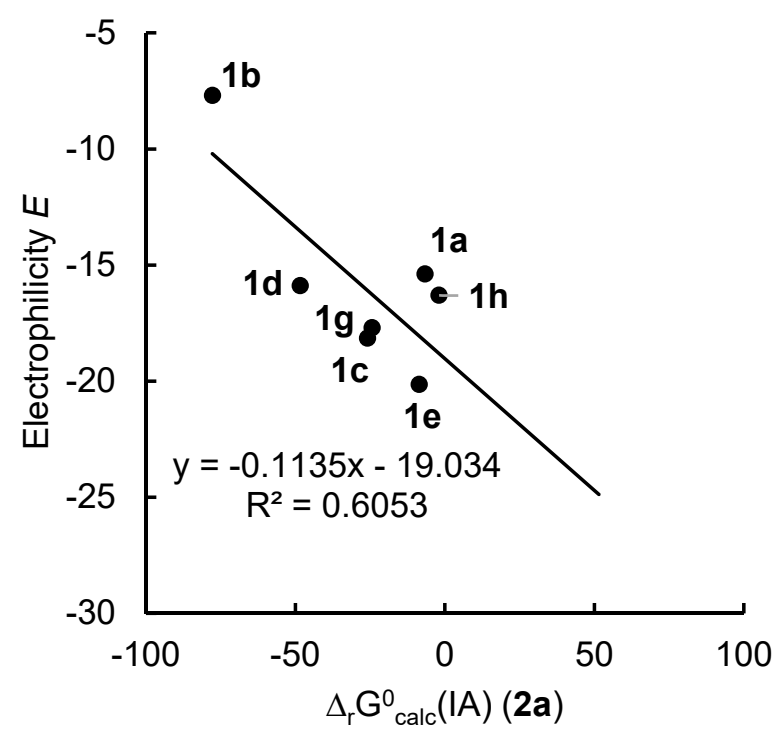

B

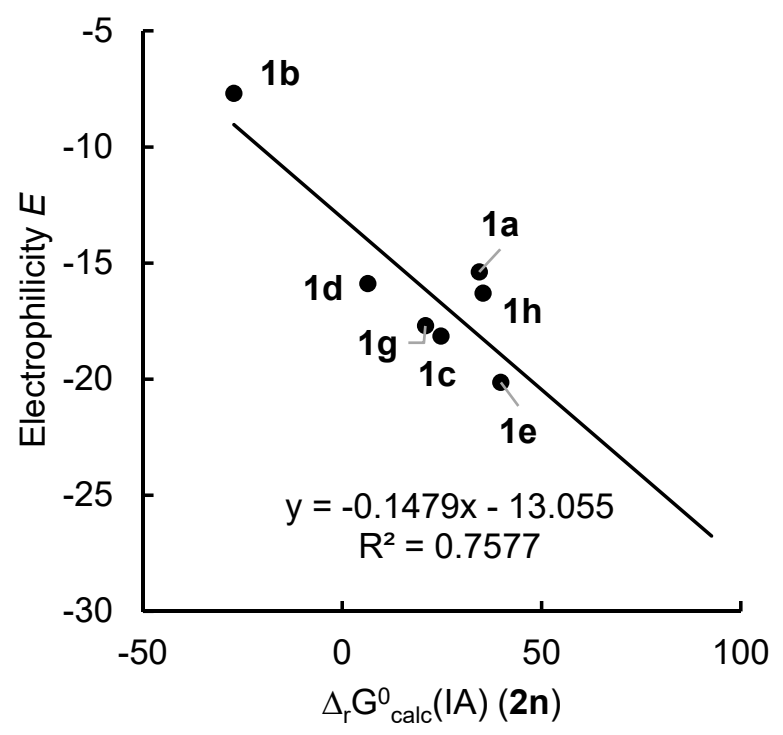

C

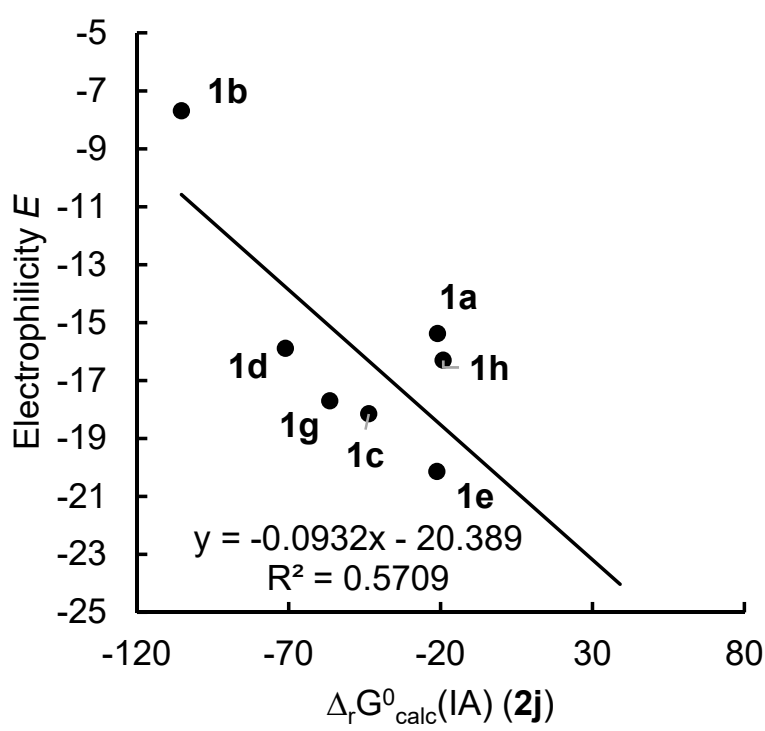

Figure S24. Correlation between the experimental $E$ and Gibbs reaction energies $\Delta_{\mathrm{r}} \mathrm{G}^{0} \mathrm{calc}(\mathbf{I A})\left(\mathrm{kJ}^{\mathrm{mol}}{ }^{-1}\right)$ for the reactions of heteroallenes $\mathbf{1}$ with 2 $(\mathbf{2 a}, \mathbf{n}, \mathbf{j})$ calculated at the IEFPCM(DMSO)/B3LYP-D3/6-311+G(d,p) level of theory. 
A

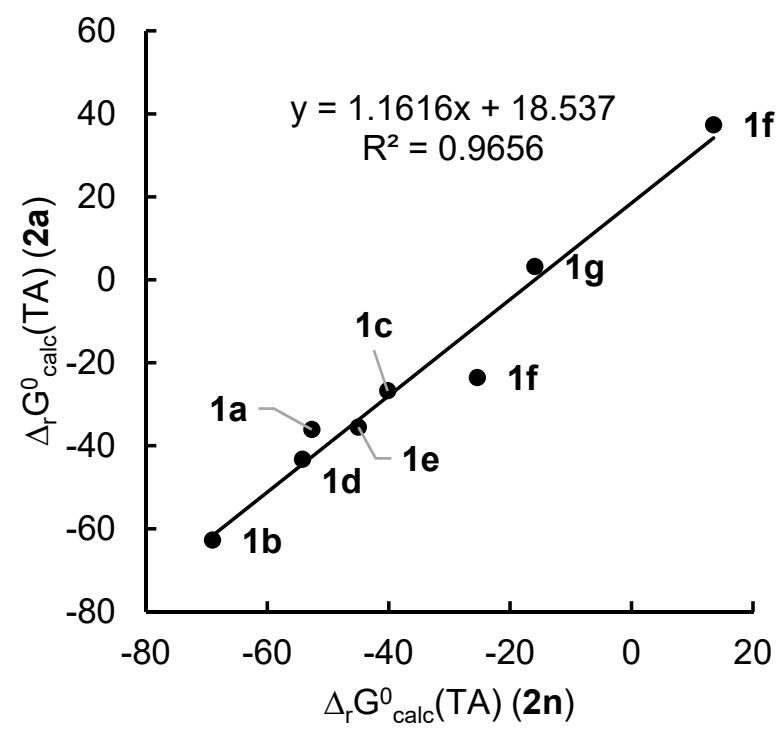

B

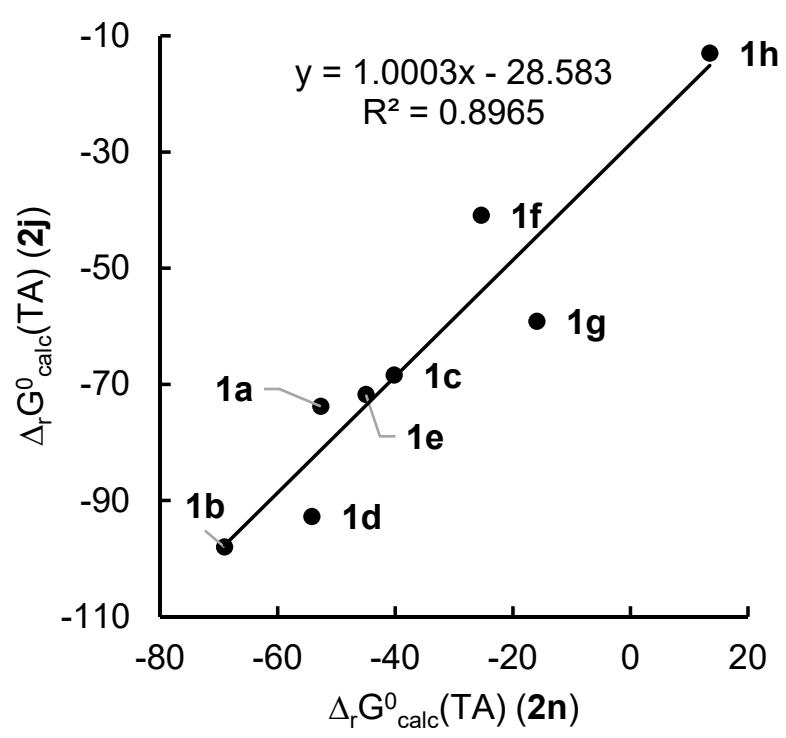

C

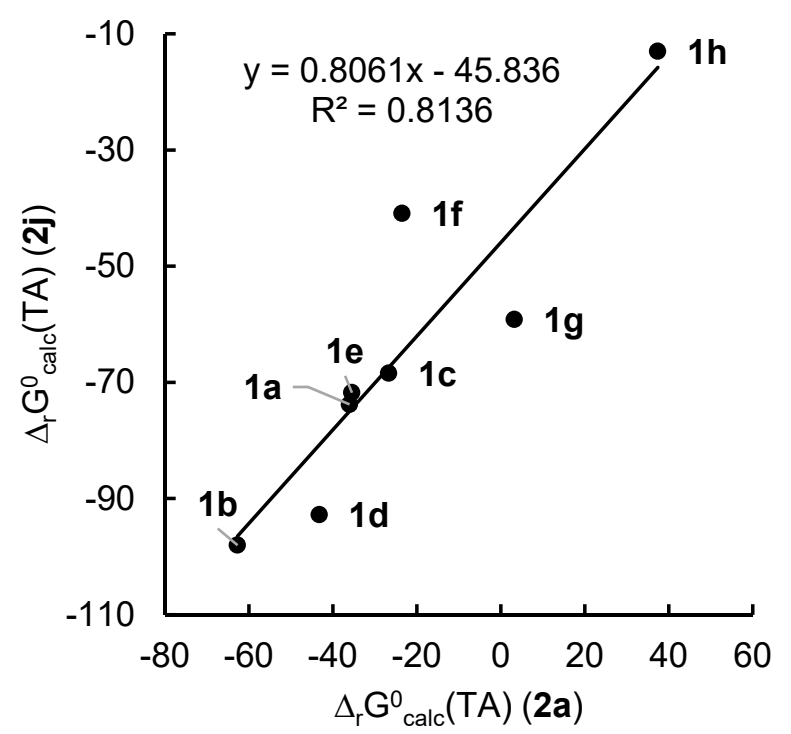

Figure S25. Correlation between Gibbs reaction energies $\Delta_{\mathrm{r}} \mathrm{G}^{0}$ calc $(\mathbf{T A})\left(\mathrm{kJ} \mathrm{mol}^{-1}\right)$ for the reactions of heteroallenes 1 with different carbanions 2 $(\mathbf{2 a}, \mathbf{n}, \mathbf{j})$ calculated at the IEFPCM(DMSO)/B3LYP-D3/6-311+G(d,p) level of theory. 
A

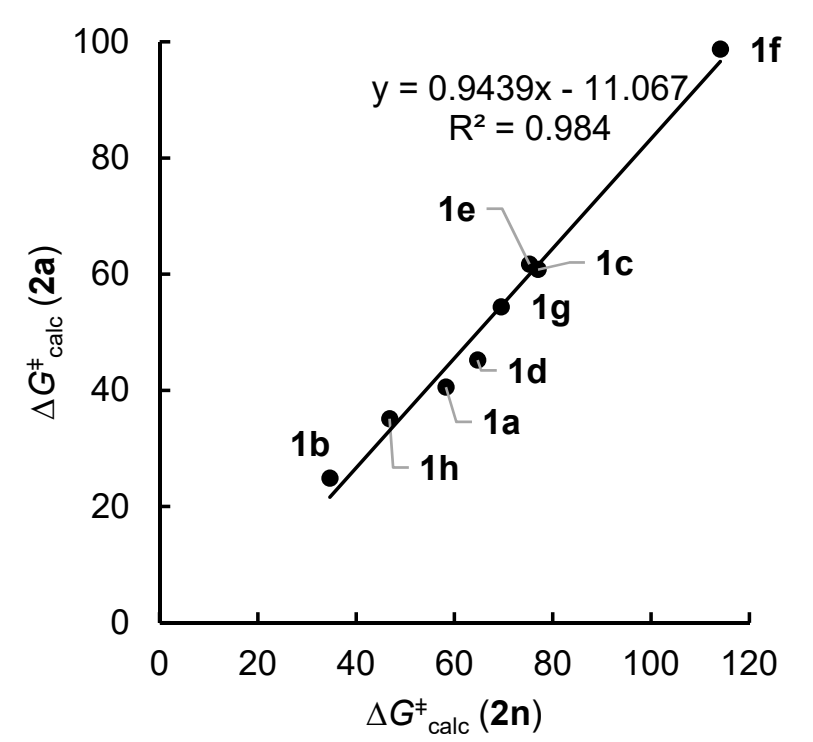

B

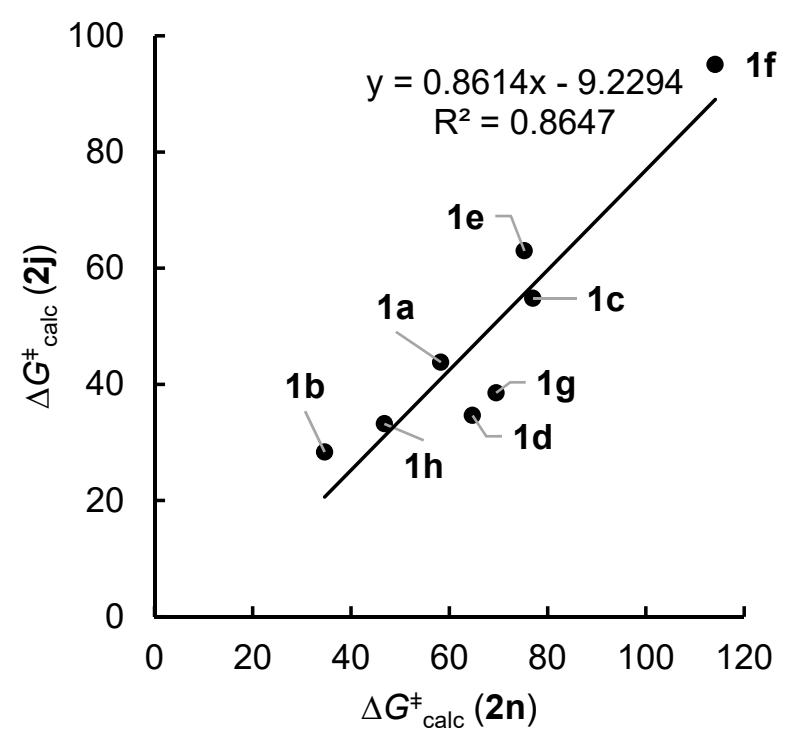

C

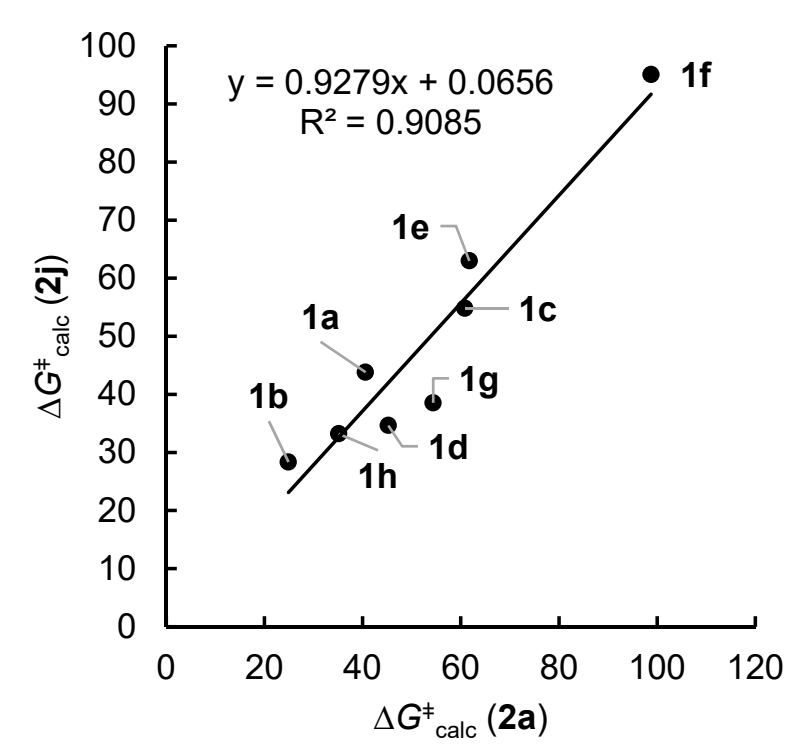

Figure S26. Correlation between Gibbs energies of activation $\Delta G_{\text {calc }}^{\ddagger}\left(\mathrm{kJ} \mathrm{mol}^{-1}\right)$ for the reactions of heteroallenes $\mathbf{1}$ with different carbanions $\mathbf{2}(\mathbf{2 a}, \mathbf{n}, \mathbf{j})$ calculated at the IEFPCM(DMSO)/B3LYP-D3/6-311+G(d,p) level of theory. 
A

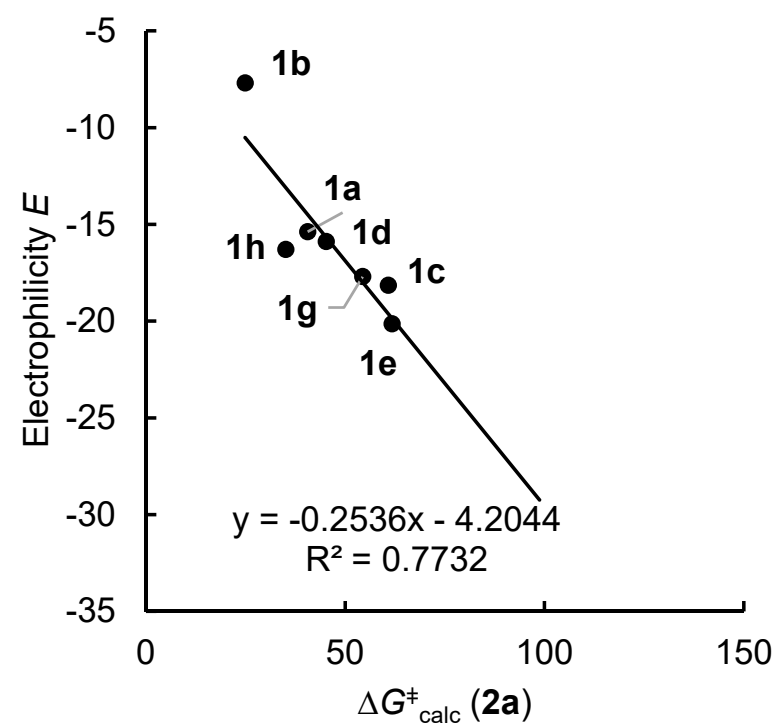

B

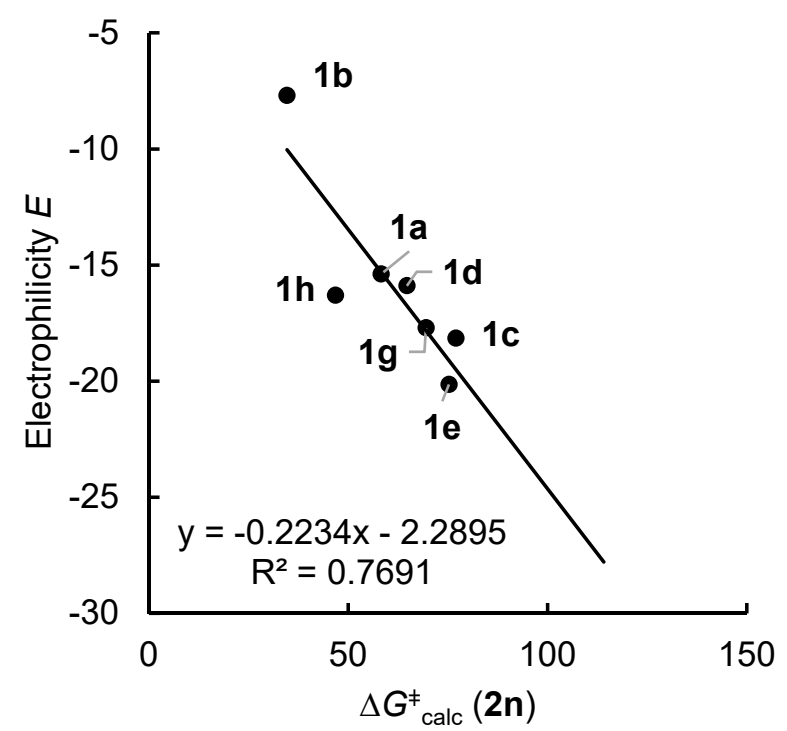

C

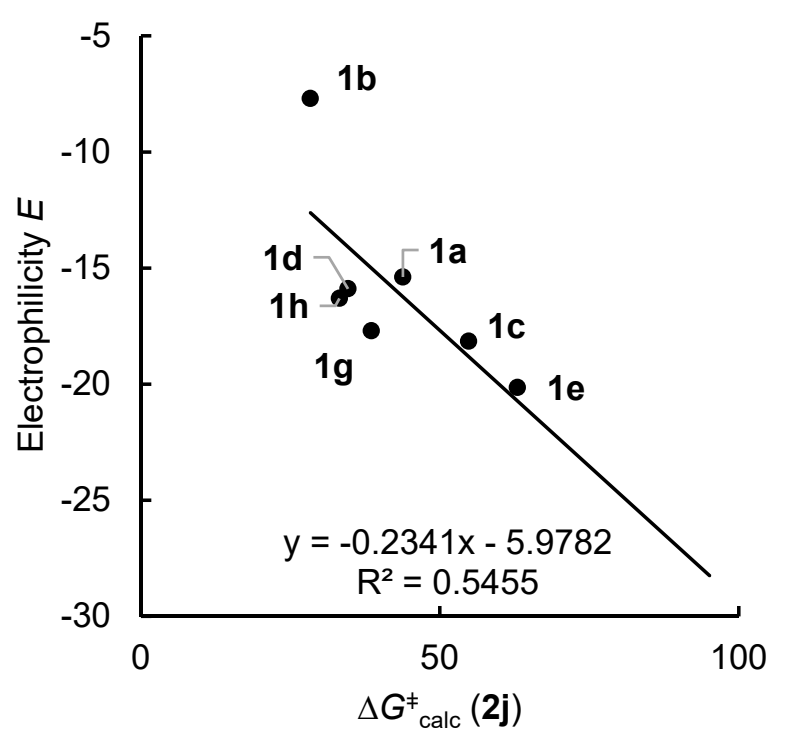

Figure S27. Correlation between the experimental electrophilicities $E$ and Gibbs energies of activation $\Delta G^{\ddagger}$ calc $\left(\mathrm{kJ}\right.$ mol $\left.{ }^{-1}\right)$ for the reactions of heteroallenes 1 with $2(\mathbf{2 a}, \mathbf{n}, \mathbf{j})$ calculated at the IEFPCM(DMSO)/B3LYP-D3/6-311+G(d,p) level of theory. 
A

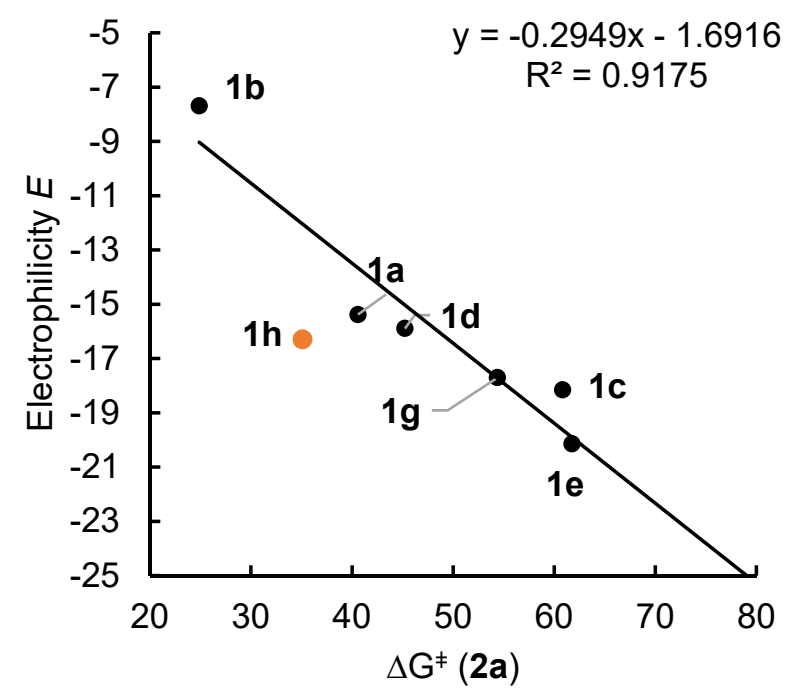

B

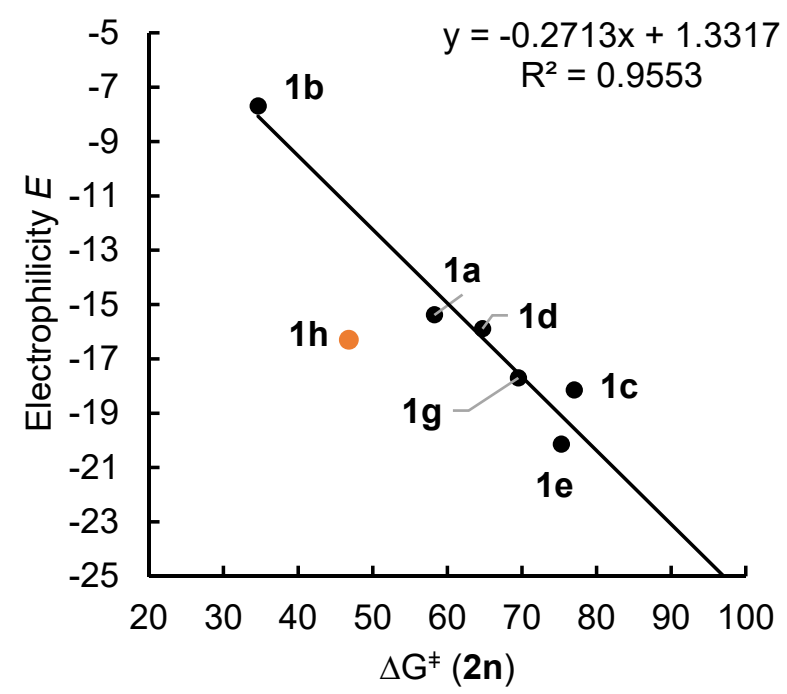

C

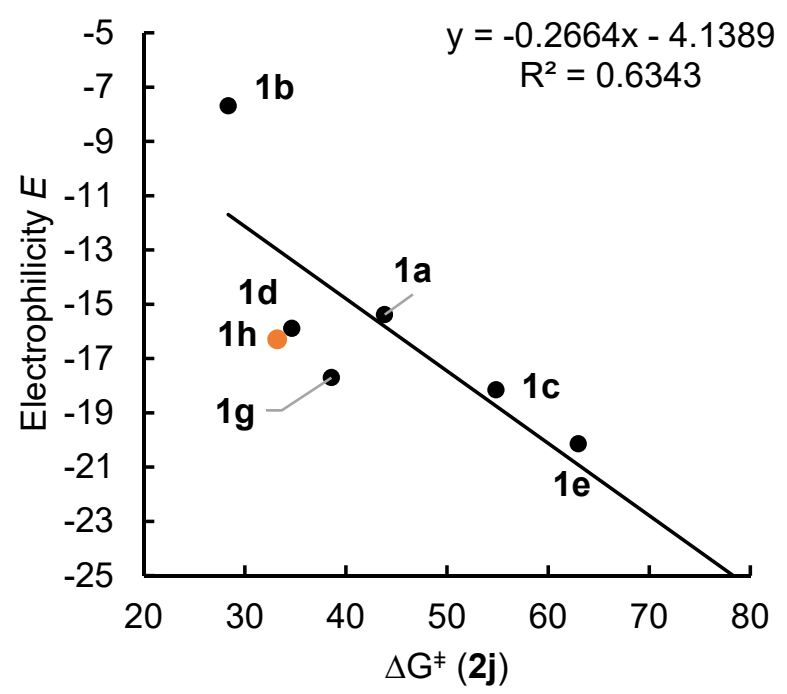

Figure S28. Correlation between the experimental electrophilicities $E$ and Gibbs energies of activation $\Delta G^{\ddagger}$ calc $\left(\mathrm{kJ}^{\mathrm{mol}}{ }^{-1}\right)$ for the reactions of heteroallenes 1 with $2(\mathbf{2 a}, \mathbf{n}, \mathbf{j})$ calculated at the IEFPCM(DMSO)/B3LYP-D3/6-311+G(d,p) level of theory (the entry for $\mathrm{CO}_{2}(\mathbf{1 h})$ was omitted when calculating the the correlation line). 
A

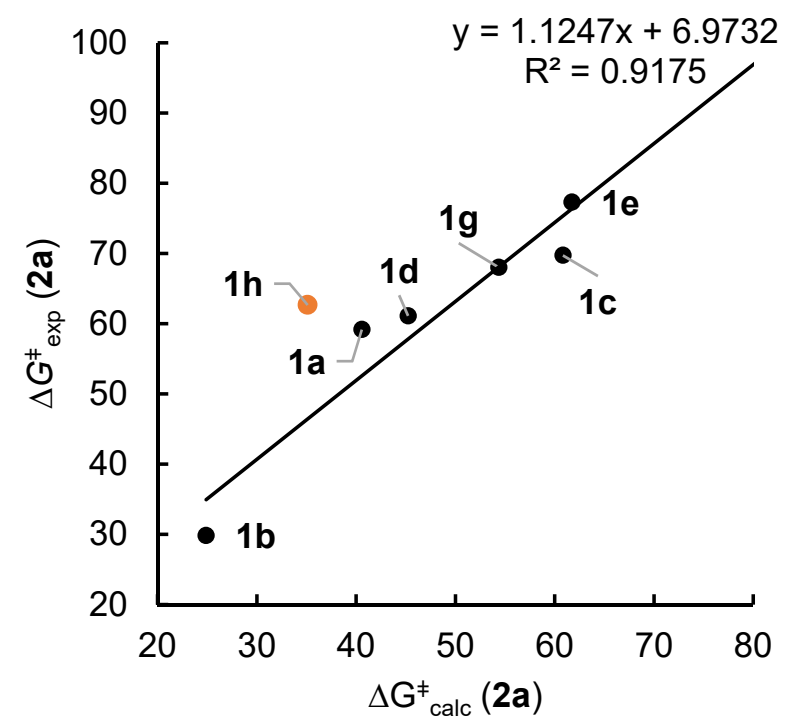

B

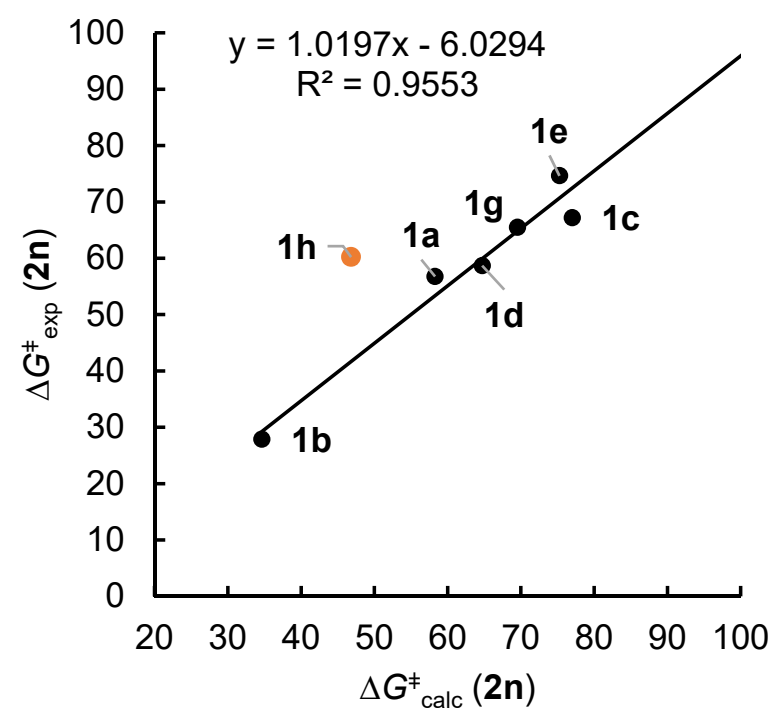

C

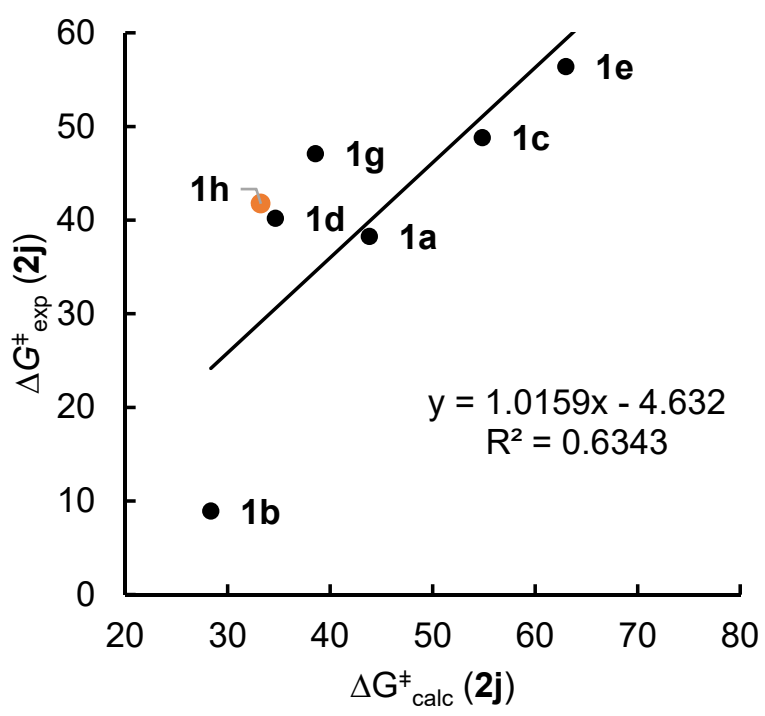

Figure S29. Correlation of experimental Gibbs energies of activation for the reactions of (2a,n,j) with $\mathbf{1}$ (from Eq 1 in the main text using $N$ and $S \mathrm{~N}$ from Table 1 and $E$ from Table 3; data for $\mathrm{CO}_{2}$ not used for the correlation) against the corresponding values calculated at the IEFPCM(DMSO)/B3LYP-D3/6-311+G(d,p) level of theory. 
A

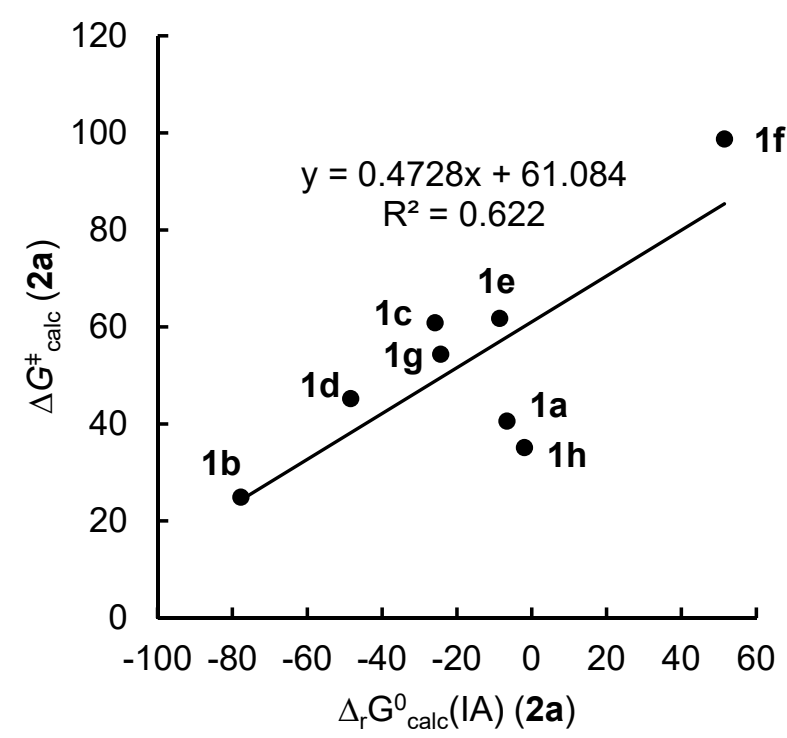

B

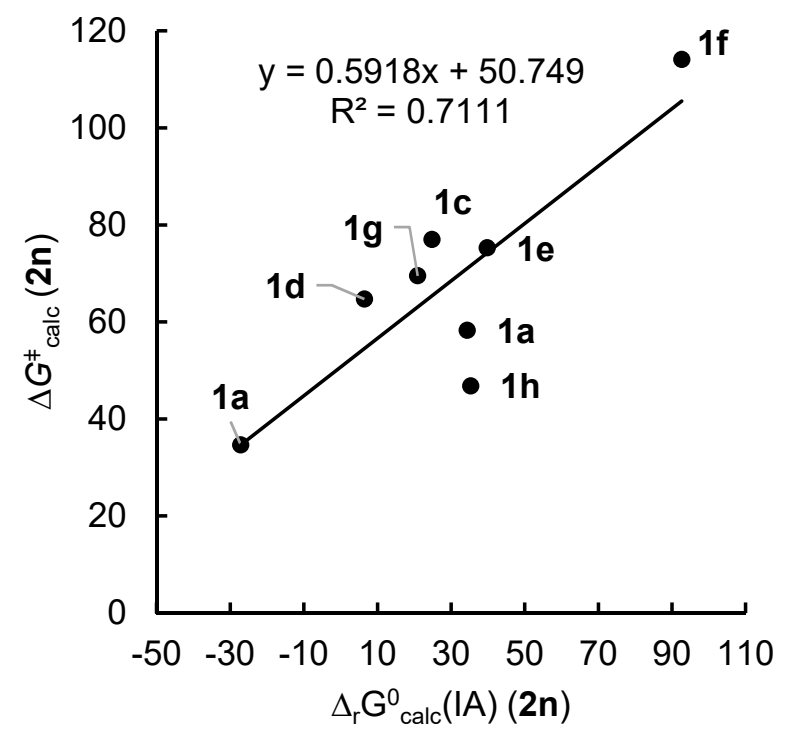

C

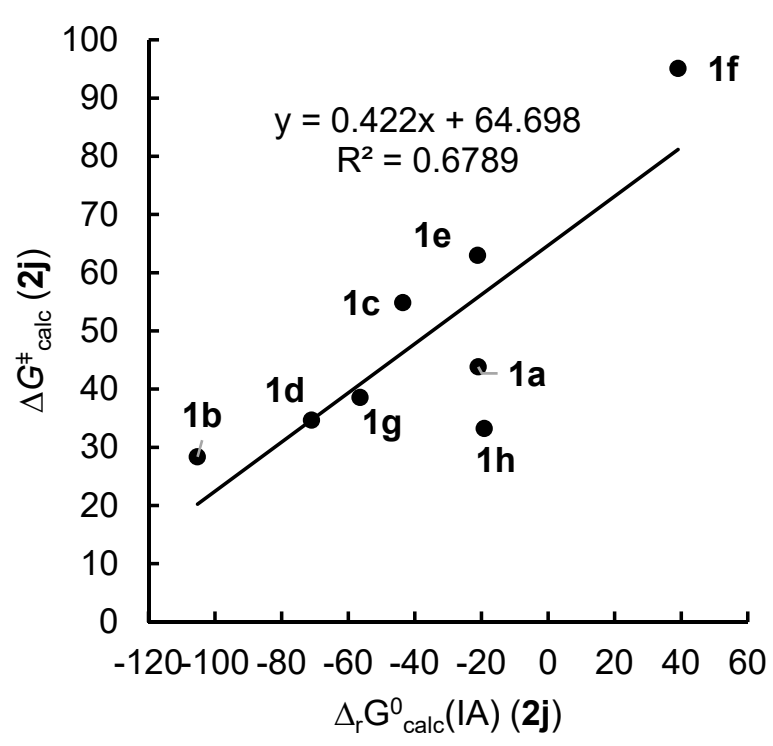

Figure S30. Correlation between Gibbs energies of activation $\Delta \mathrm{G}^{\ddagger}\left(\mathrm{kJ} \mathrm{mol}^{-1}\right)$ vs Gibbs reaction energies $\Delta_{\mathrm{r}} \mathrm{G}^{0}($ IA $)\left(\mathrm{kJ}\right.$ mol $\left.{ }^{-1}\right)$ for the reactions of heteroallenes 1 with $2(\mathbf{2 a}, \mathbf{n}, \mathbf{j})$ calculated at the IEFPCM(DMSO)/B3LYP-D3/6-311+G(d,p) level of theory. 


\section{Potential Energy Surface for the Reaction of 1b with Enol Ether 30}

In order to get a more detailed insight into the reaction mechanism of the cyclization reaction of $\mathbf{1 b}$ with ethyl vinyl ether (3o) in $\mathrm{CH}_{2} \mathrm{Cl}_{2}$, we studied the potential energy surface (PES) of the reaction with the B3LYP-D3/6-311+G(d,p) method considering dichloromethane solvation by the IEFPCM model.
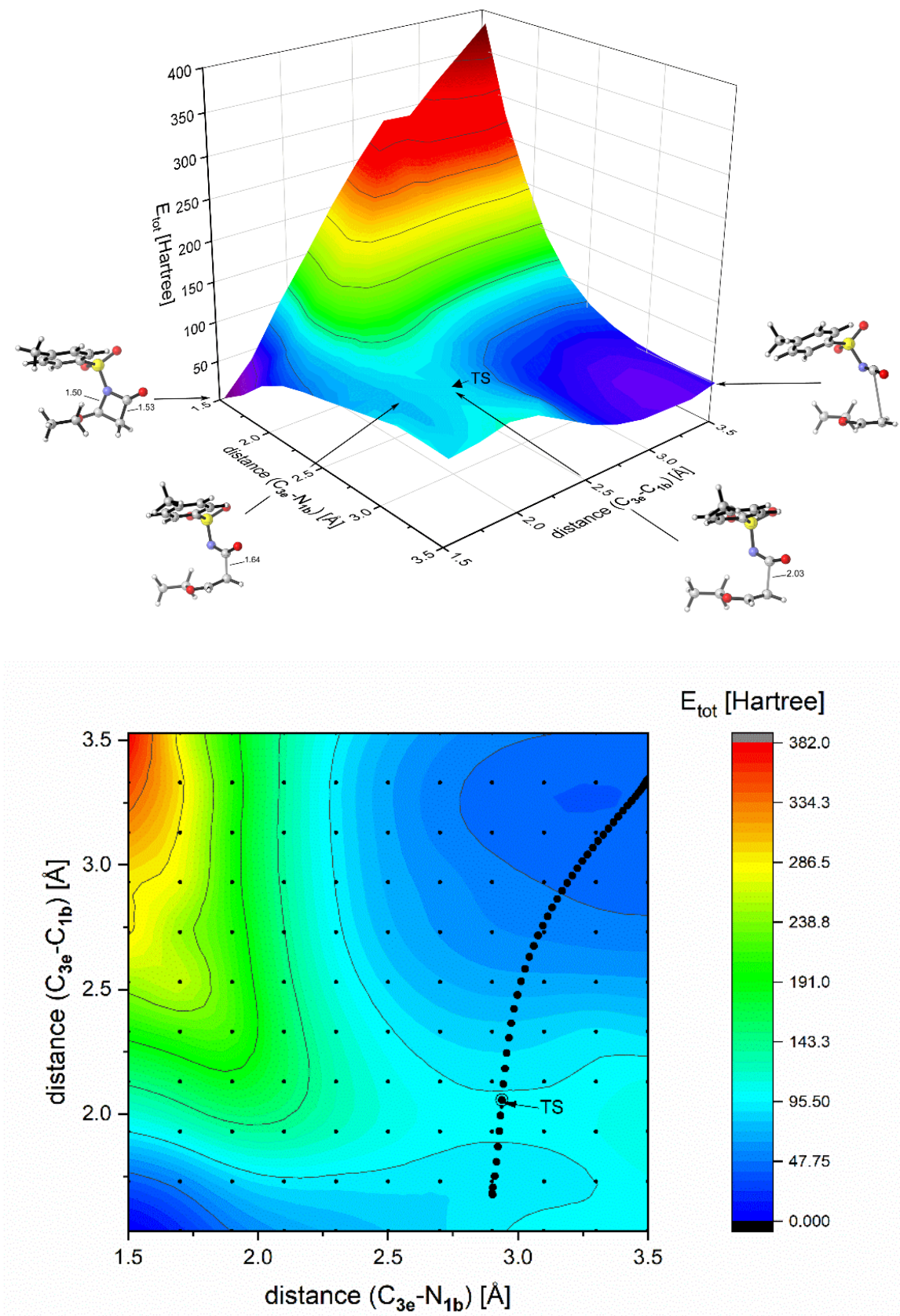

Figure S31. (Top) Potential energy surface for the reaction of $\mathbf{1 b}$ with $\mathbf{3 0}$ calculated with at the B3LYP-D3/6-311+G(d,p) level of theory in dichloromethane. (Bottom) Contour plot highlighting the transition state and the reaction pathway as determined by an IRC calculation starting from the transition state. 
On the PES only one saddle-point region can be found which corresponds to the transition state for the formation of the $\mathrm{C}(\mathrm{O})-\mathrm{C}$ bond. The activation energy to reach this transition state $\Delta G^{\ddagger}$ calcd $\left(87.4 \mathrm{~kJ} \mathrm{~mol}^{-1}\right)$ is in good agreement with experiment $\left(\Delta G^{\ddagger} \exp =88.3 \mathrm{~kJ} \mathrm{~mol}{ }^{-1}\right)$. However, after passing the transition state no stable intermediate is obtained. As the PES is still curved, subsequent rotation around the $\mathrm{C}-\mathrm{C}$ bond followed by ring closure is observed. The reaction mechanism for the reaction of $\mathbf{1 b}$ with 3o, therefore, can be described as an asynchronous concerted pathway as the reaction proceeds in two steps but without a distinct intermediate. 
9. NMR Spectra

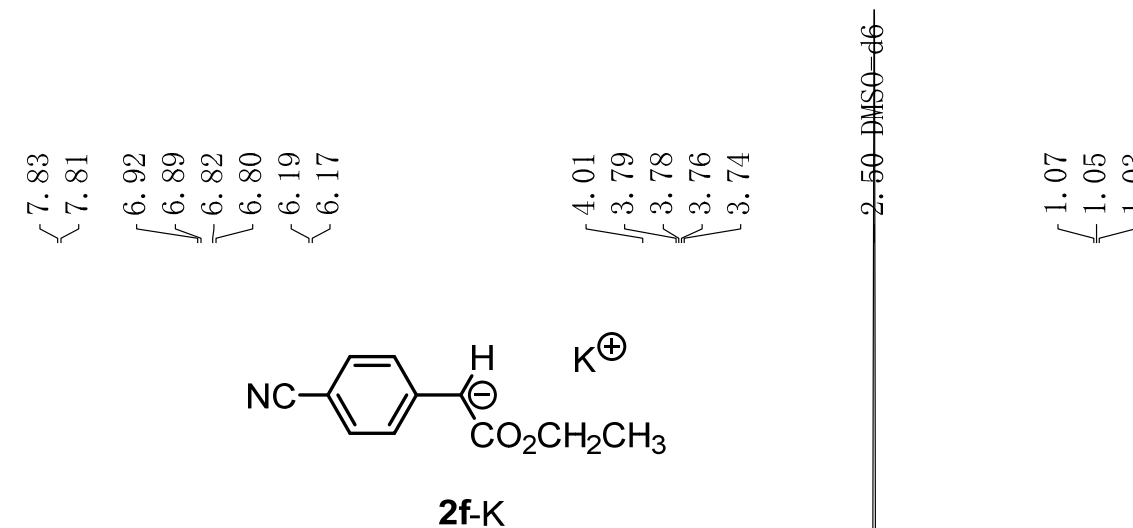

${ }^{1} \mathrm{H}$ NMR (400 MHz, $d_{6}$-DMSO)

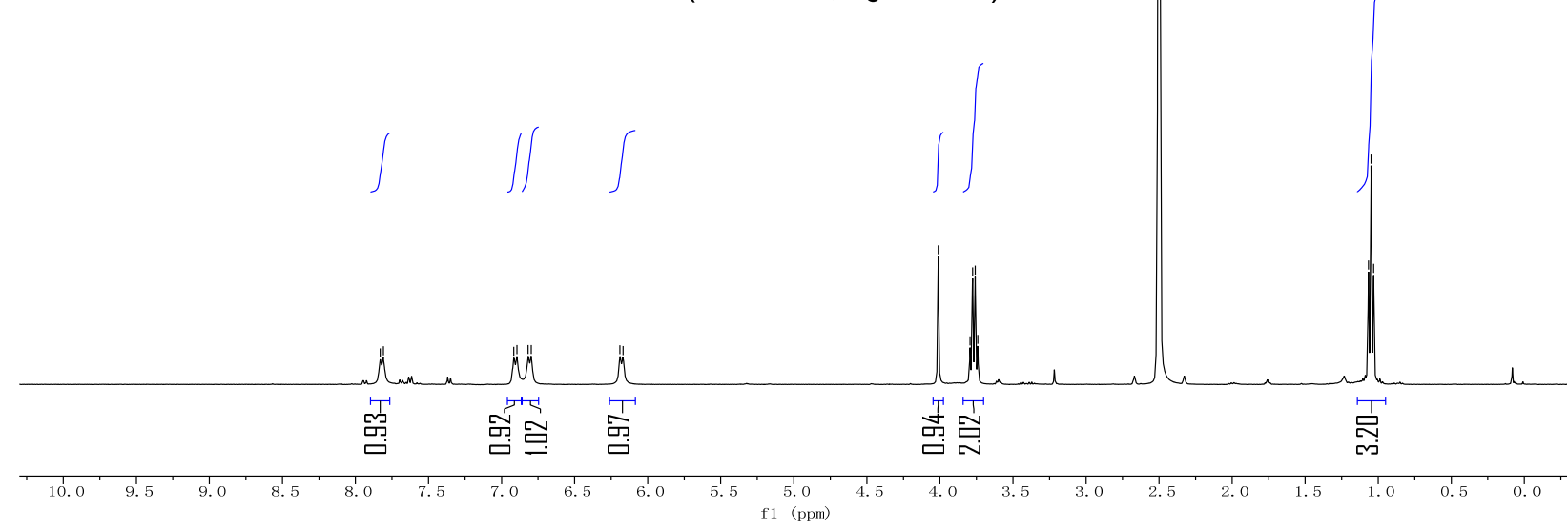

$$
\stackrel{0}{\dot{0}}
$$$$
\text { 2f-K }
$$

${ }^{13} \mathrm{C}$ NMR (101 MHz, $d_{6}$-DMSO)

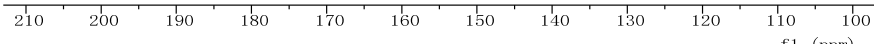




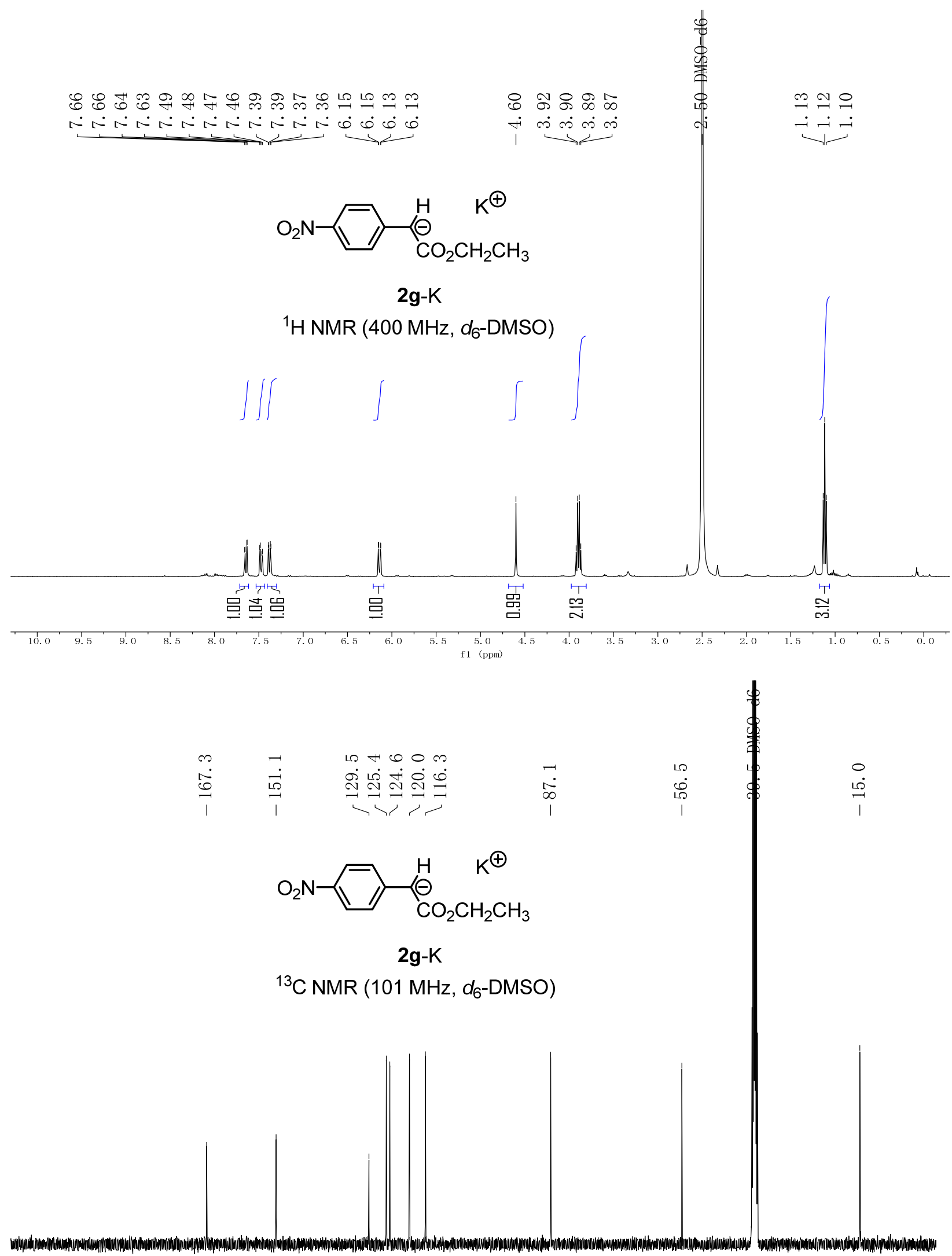

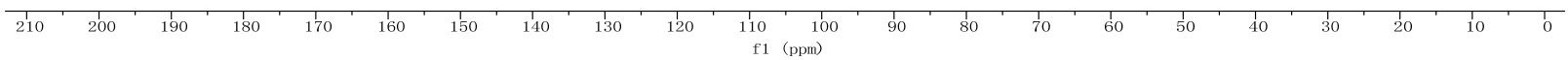




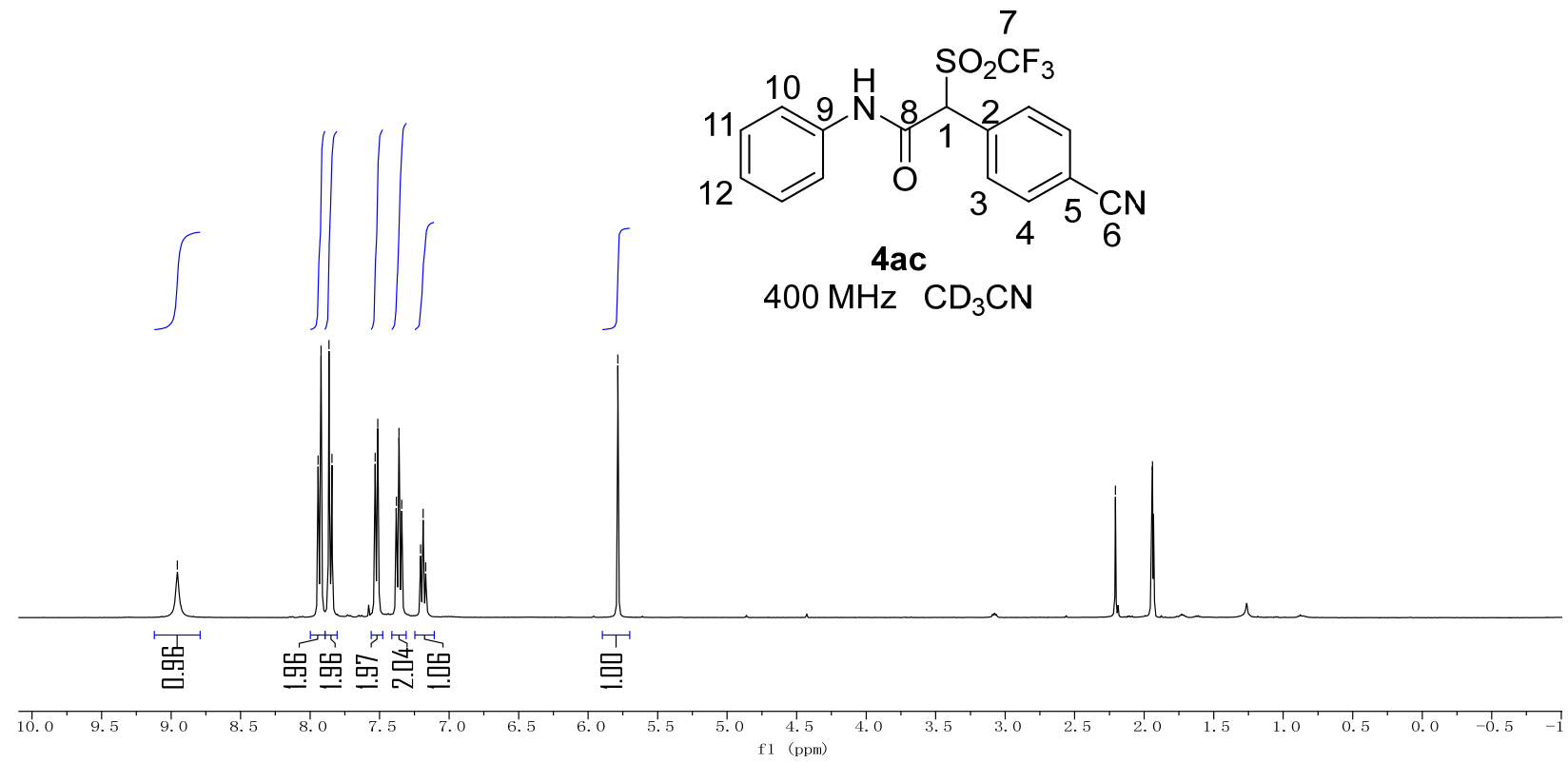

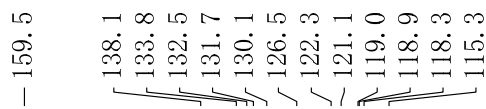
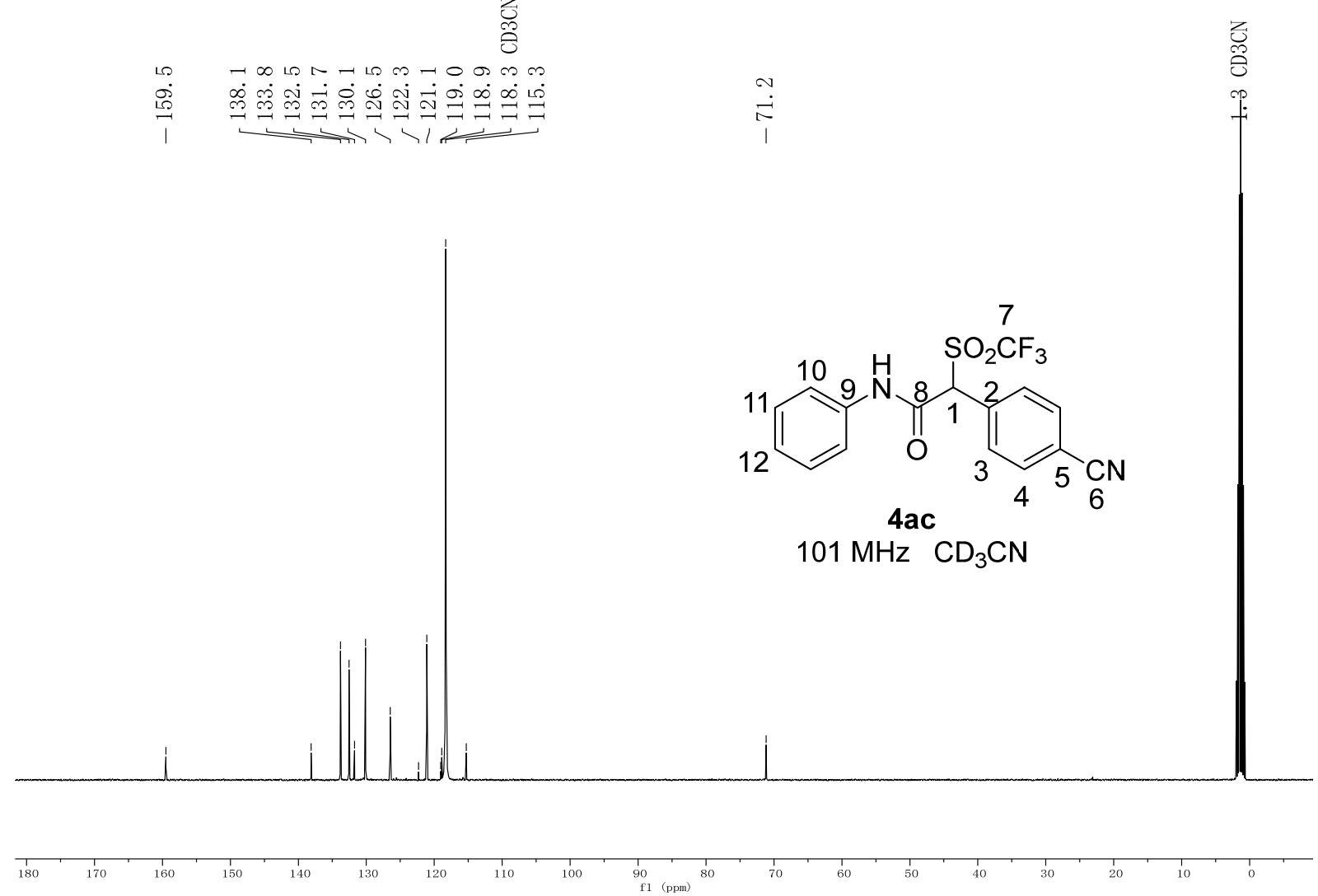


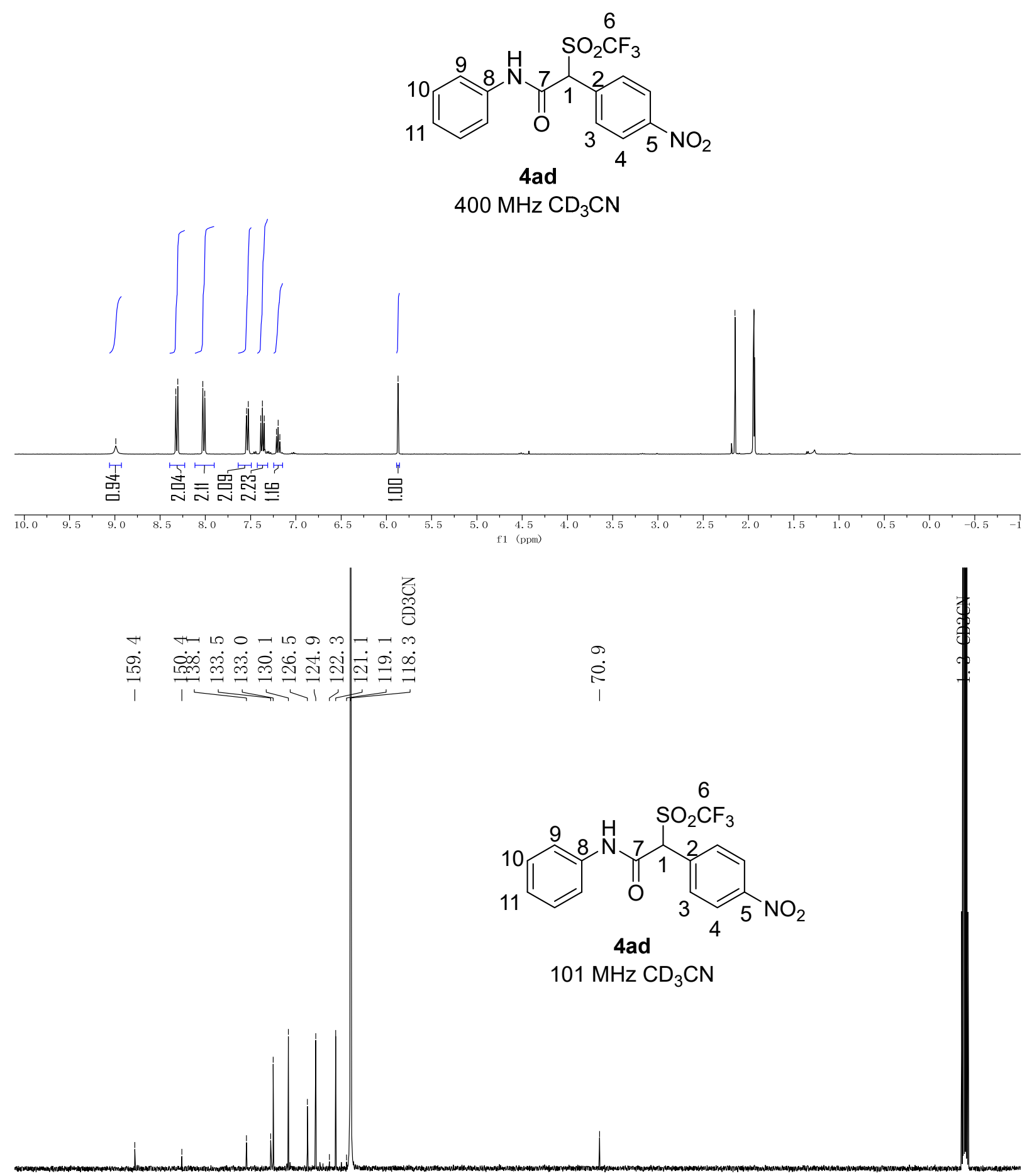



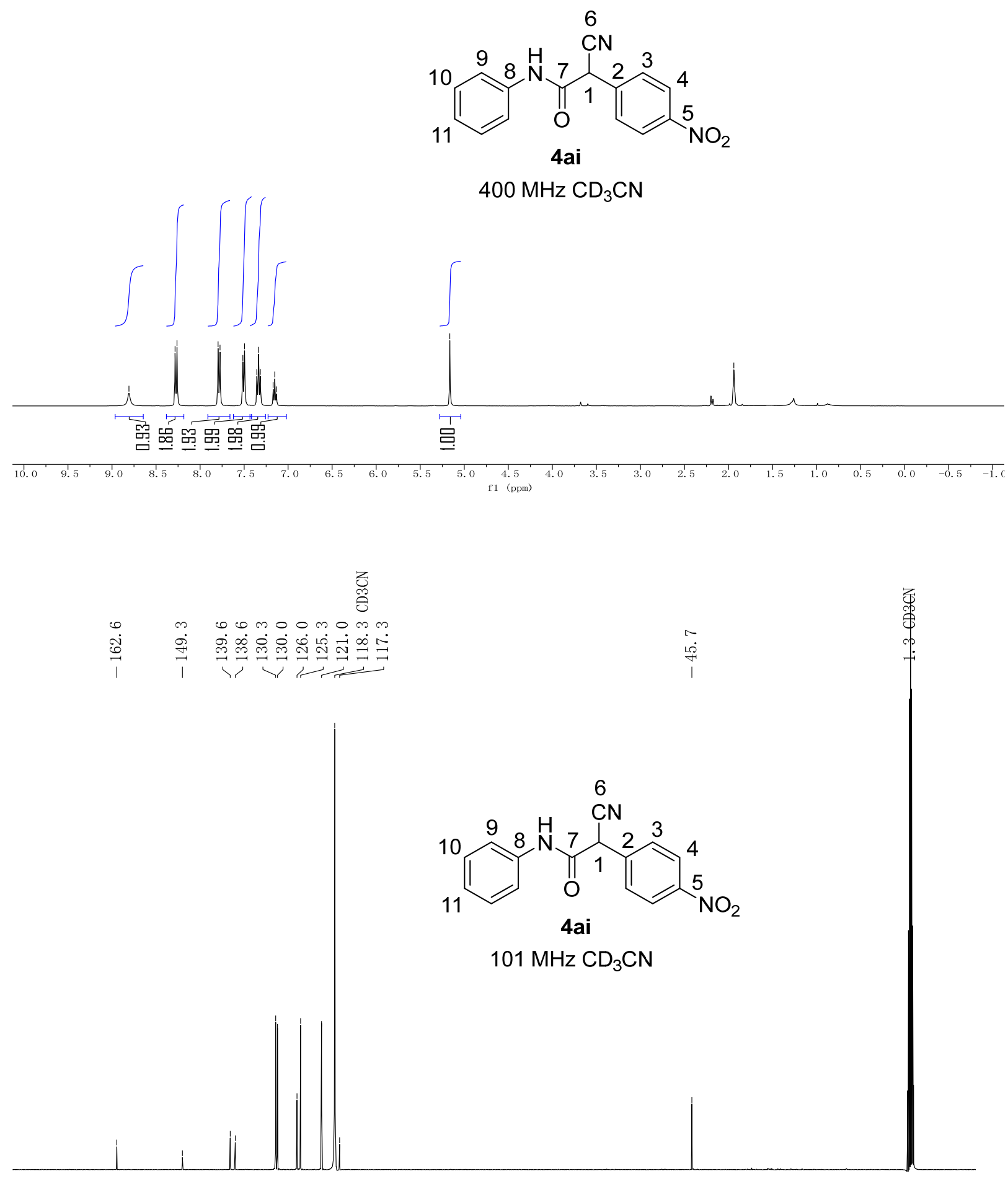


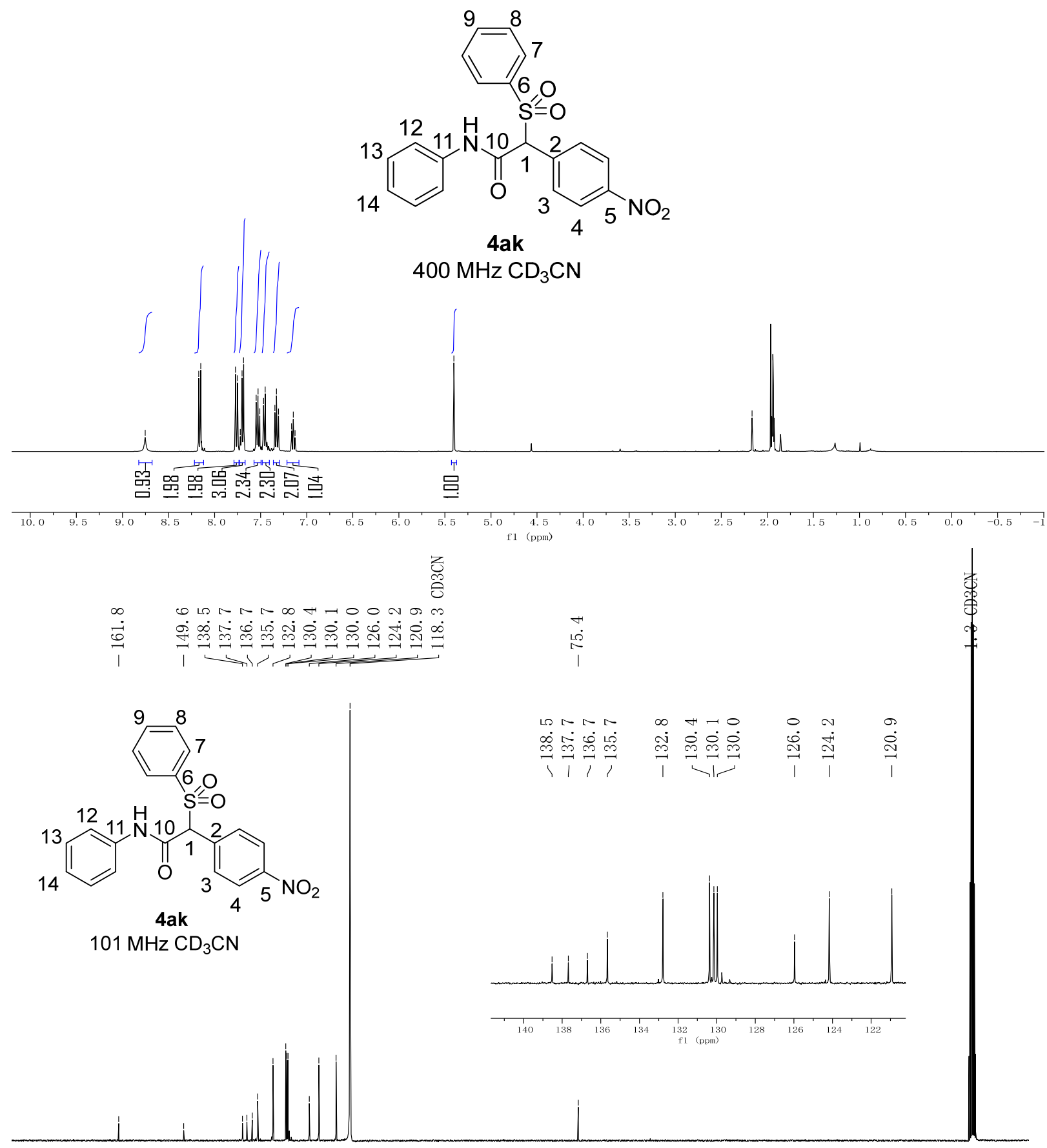


<smiles>Cc1ccc(S(=O)(=O)N[O-])cc1</smiles>

$1 b(1$ eq)<smiles>CN(OC(=O)OC(C)(C)C)C(=O)N1CCCC1</smiles>

$3 a(1 e q)$

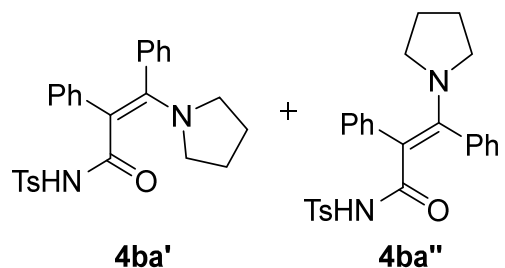

4ba'

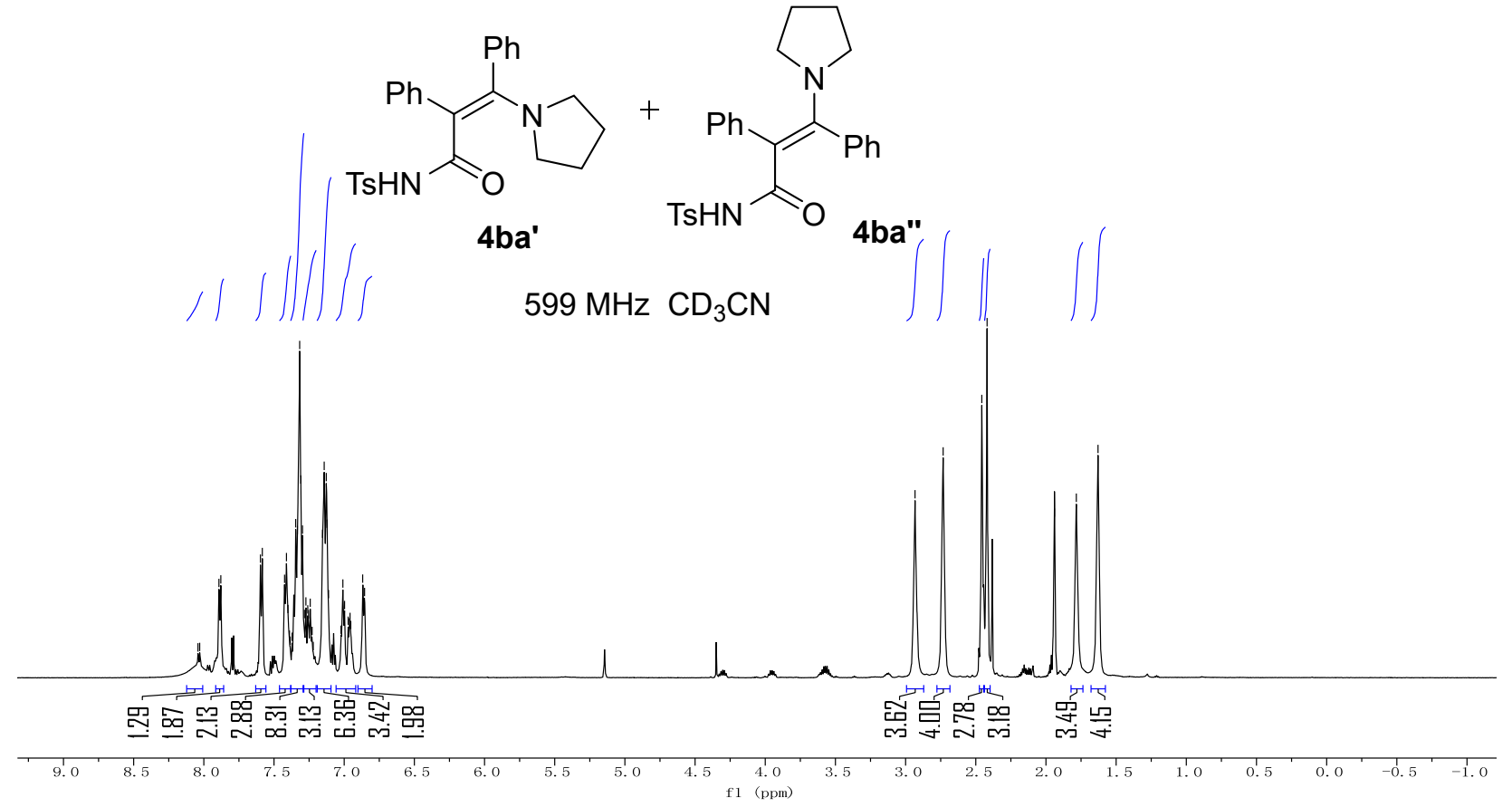




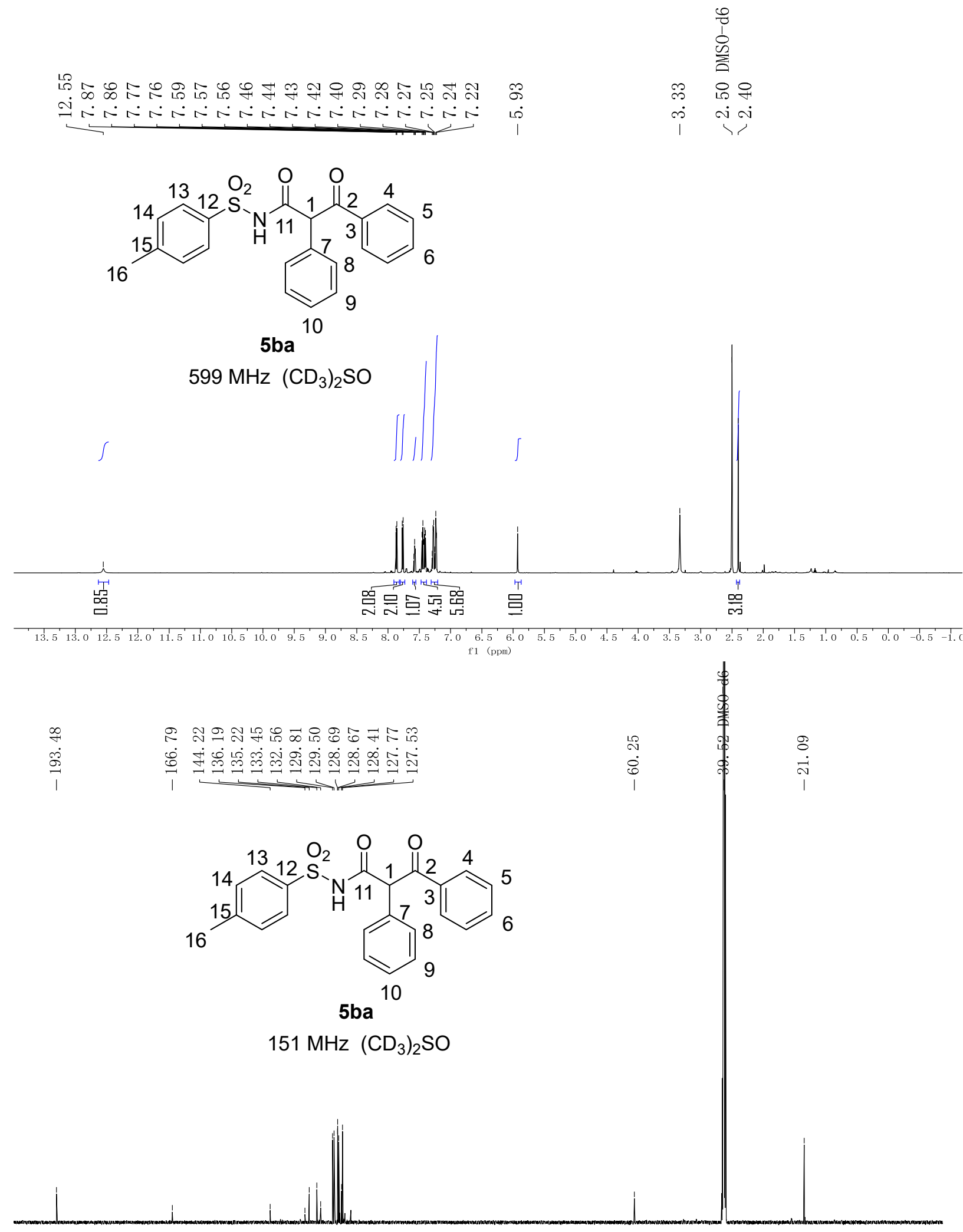




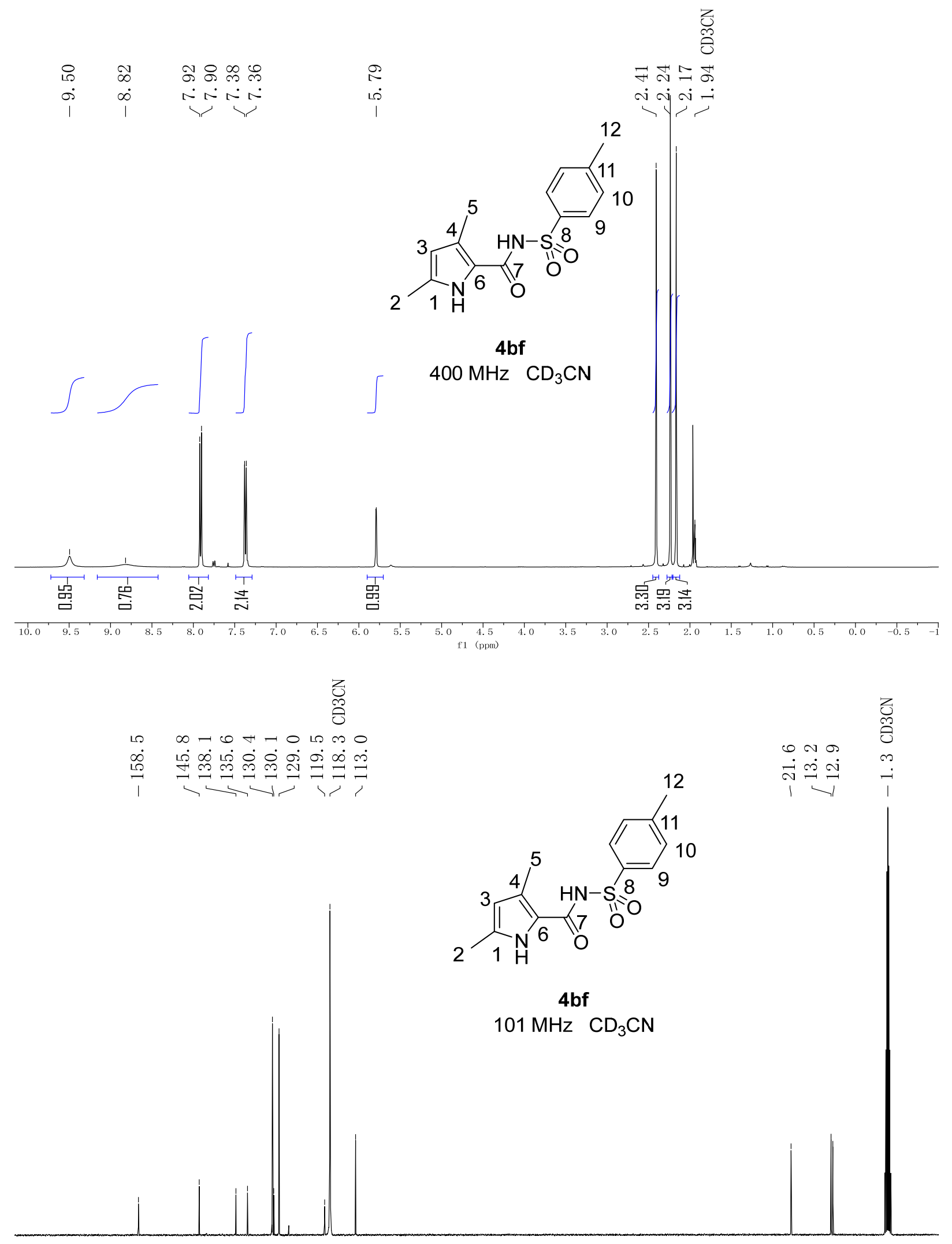


<smiles>[3H]c1ccc(NC=O)cc1</smiles><smiles>CCS(=O)(=O)N=C(C)C1=CC=CC1=Cc1ccc(N(C)C)cc1</smiles>

$400 \mathrm{MHz} \mathrm{CDCl}_{3}$

$\iiint \int d \int$
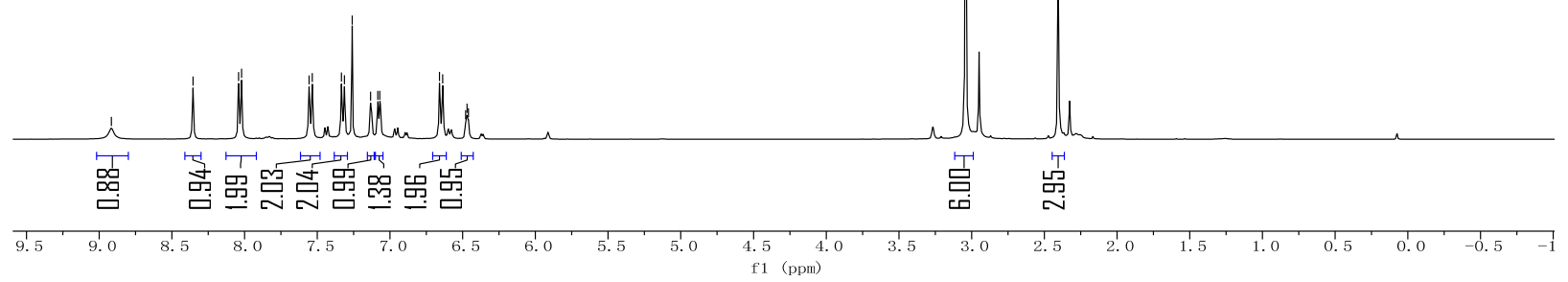

N N

ம்
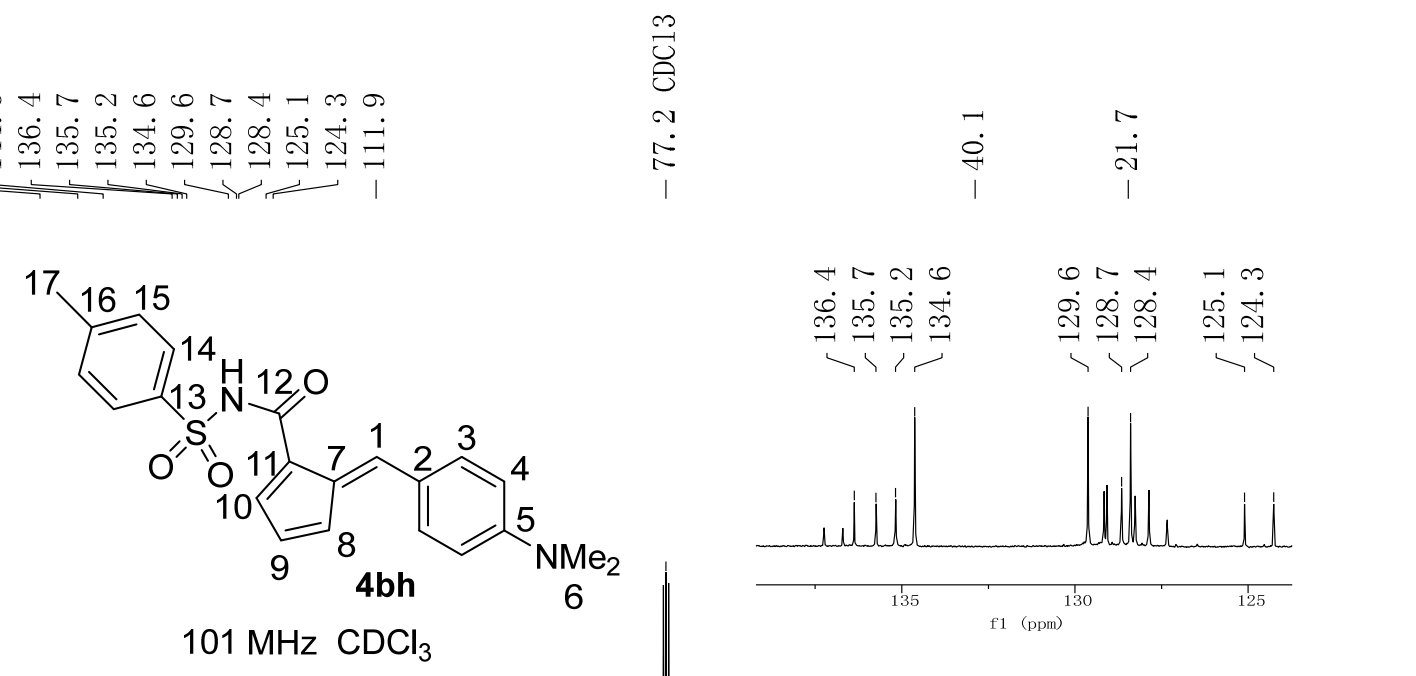

m
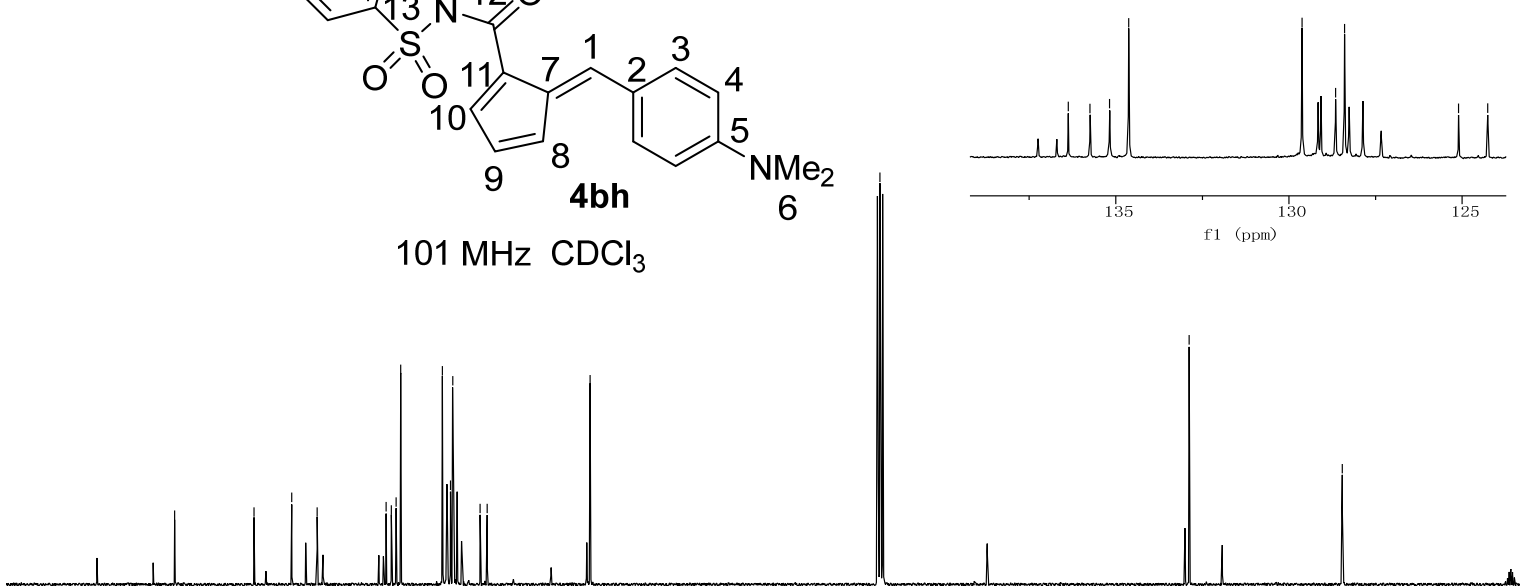
๓

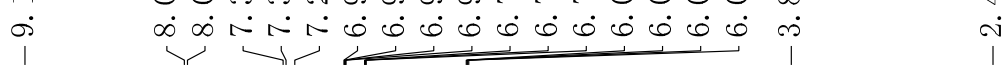

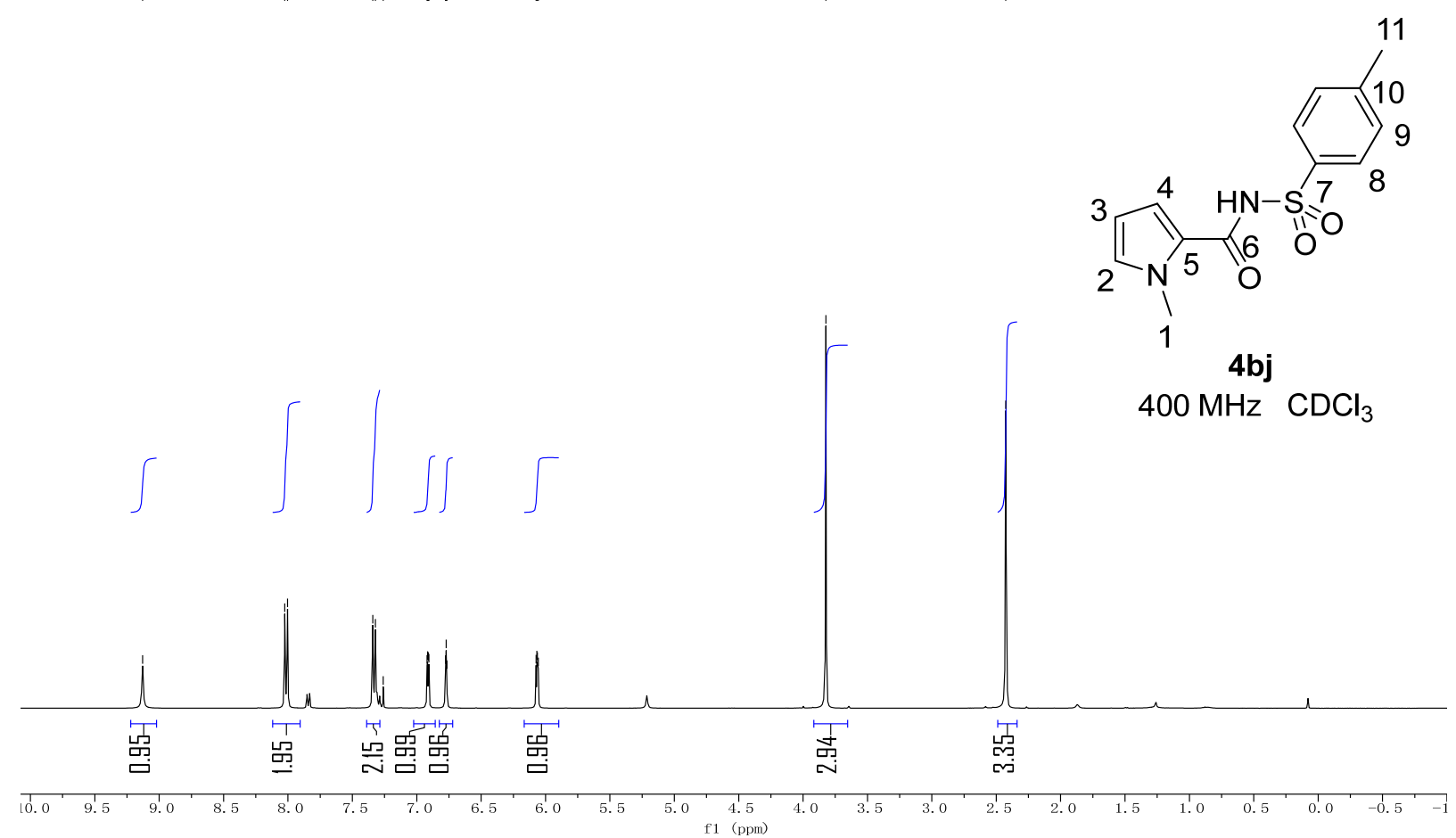

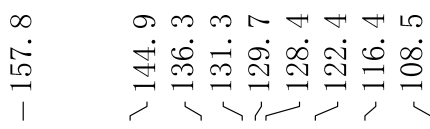
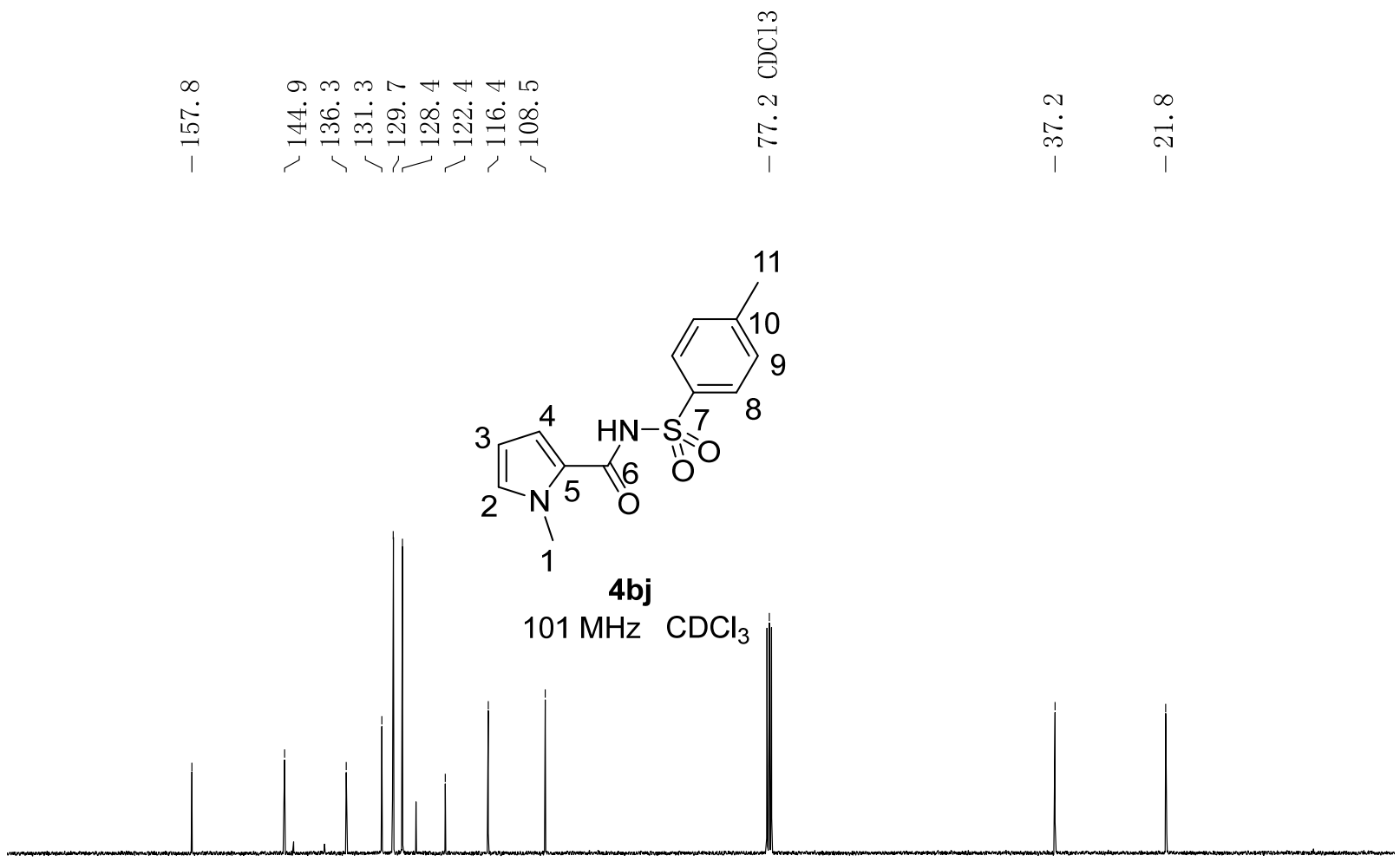
$\infty \mathbb{\infty}_{\infty}$ 우

NNハN

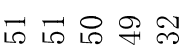

เا் ம்

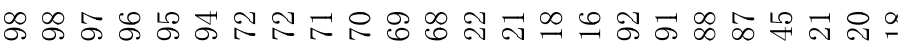

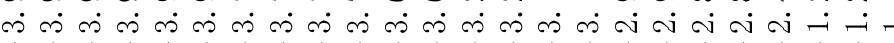

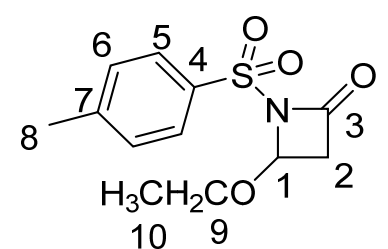

4bo

$400 \mathrm{MHz} \quad \mathrm{CD}_{2} \mathrm{Cl}_{2}$
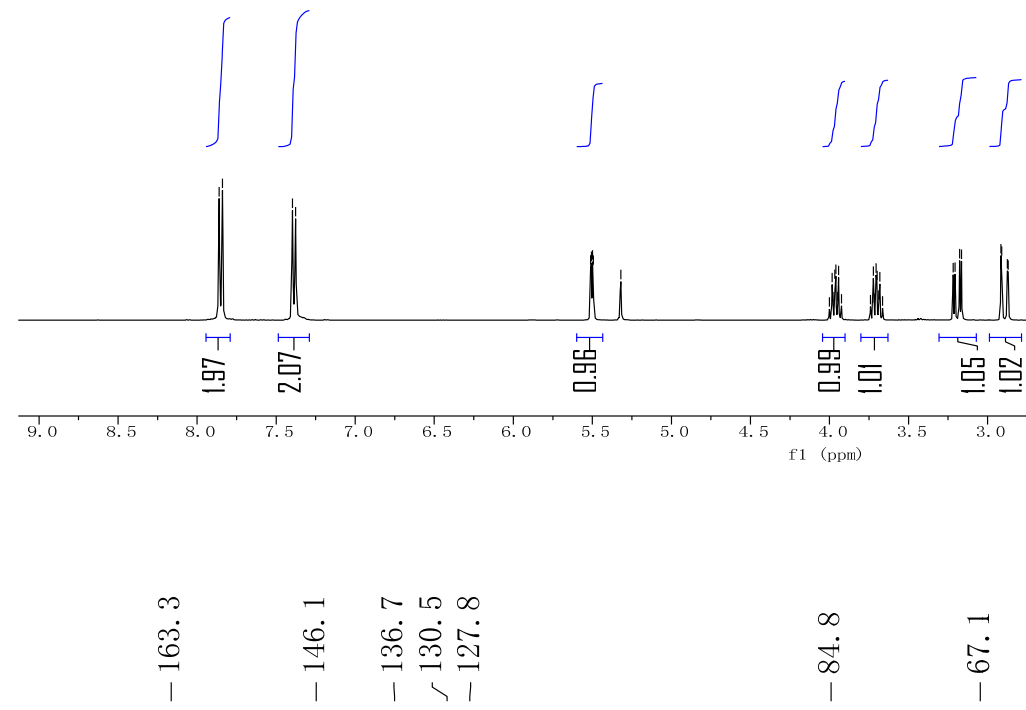

盖

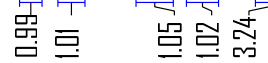

尊

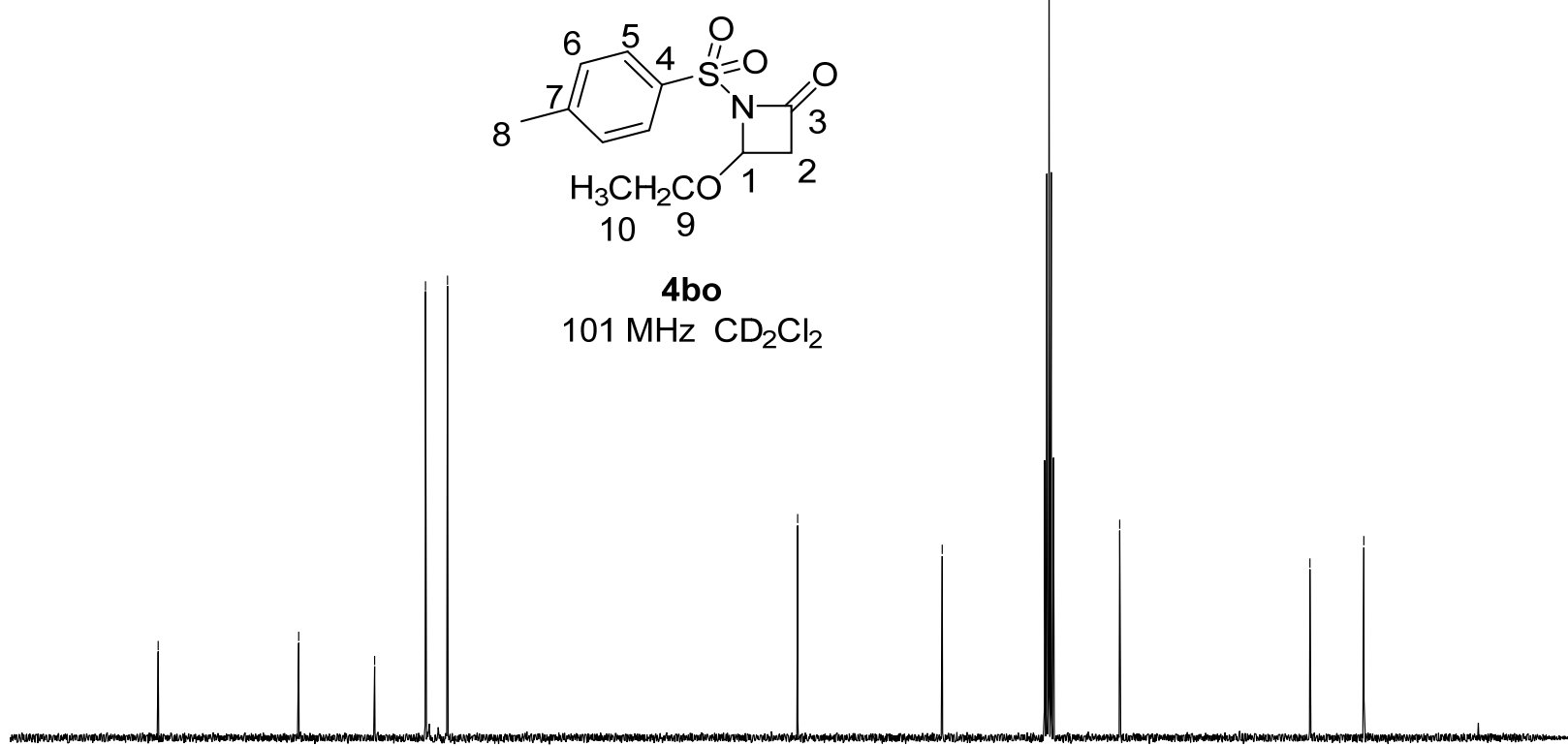

4bo $101 \mathrm{MHz} \mathrm{CD}_{2} \mathrm{Cl}_{2}$ 
ثิ

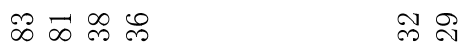

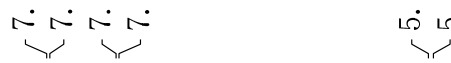

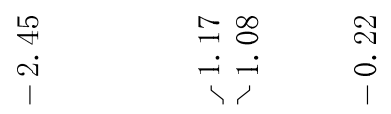

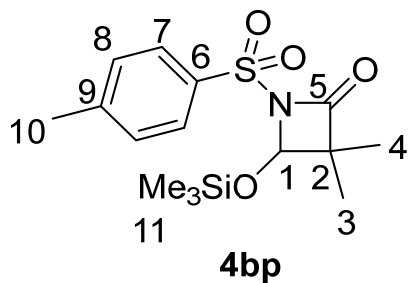

$400 \mathrm{MHz} \quad \mathrm{CD}_{2} \mathrm{Cl}_{2}$

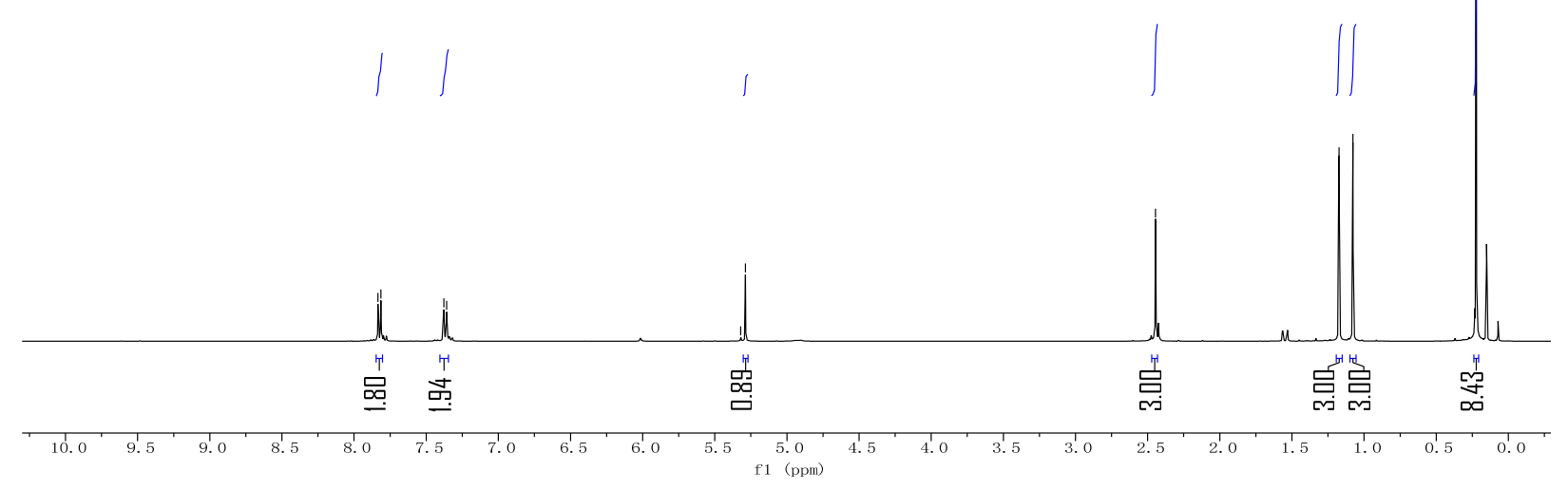

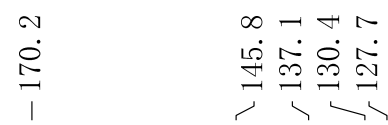

$\begin{array}{lll}\sim & 10 \\ \dot{0} & 10 & 10 \\ 1 & 10\end{array}$

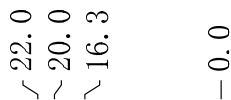

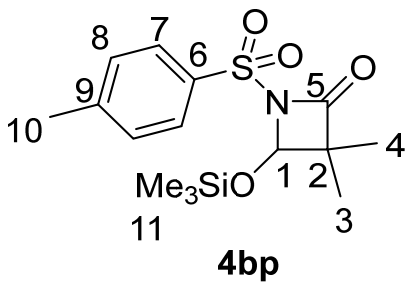

$101 \mathrm{MHz} \quad \mathrm{CD}_{2} \mathrm{Cl}_{2}$

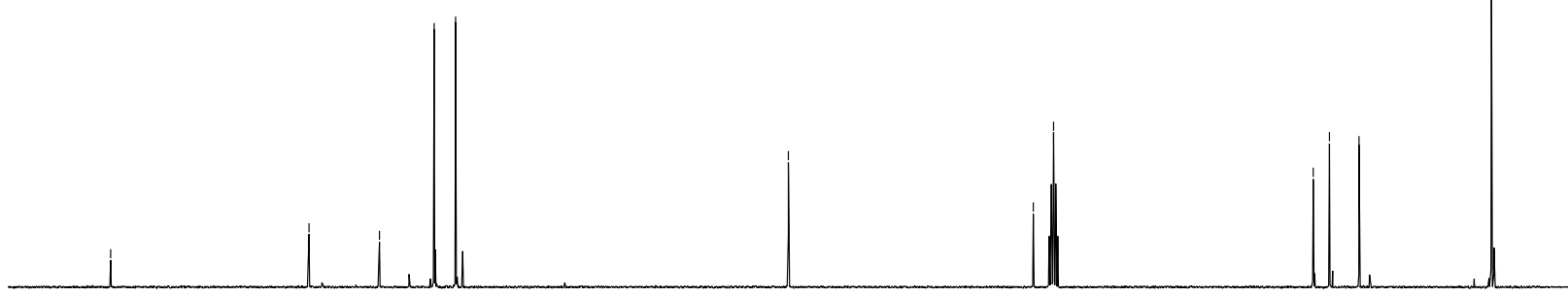


Crude reaction mixture: $1 \mathrm{c}+\mathbf{2 n}$ to give $4 \mathrm{cn}^{-} \mathrm{Na}^{+}$

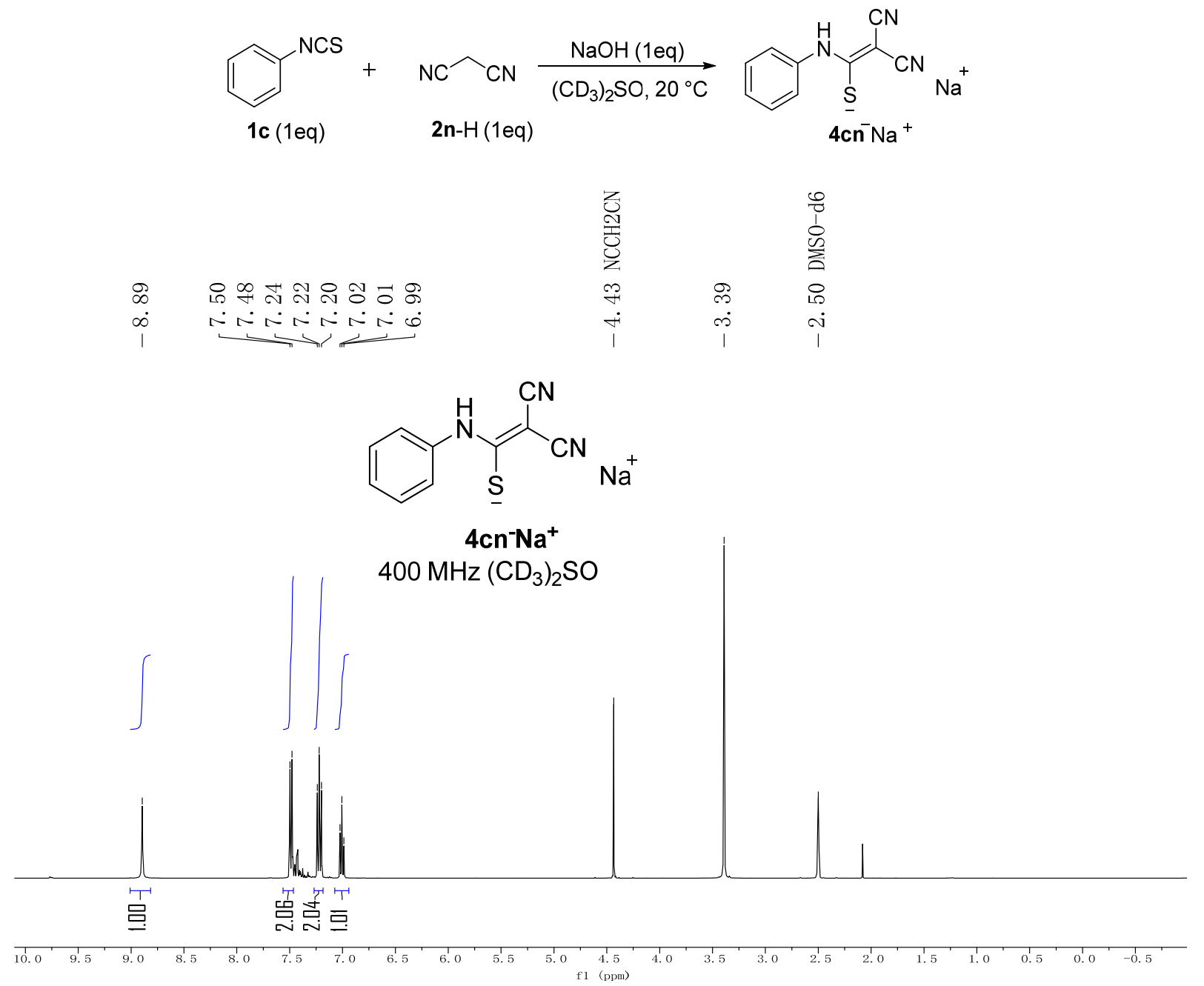


Crude reaction mixture: $\mathbf{1 c}+\mathbf{2 o}$ to give $4 \mathrm{co}^{-} \mathrm{Na}^{+}$
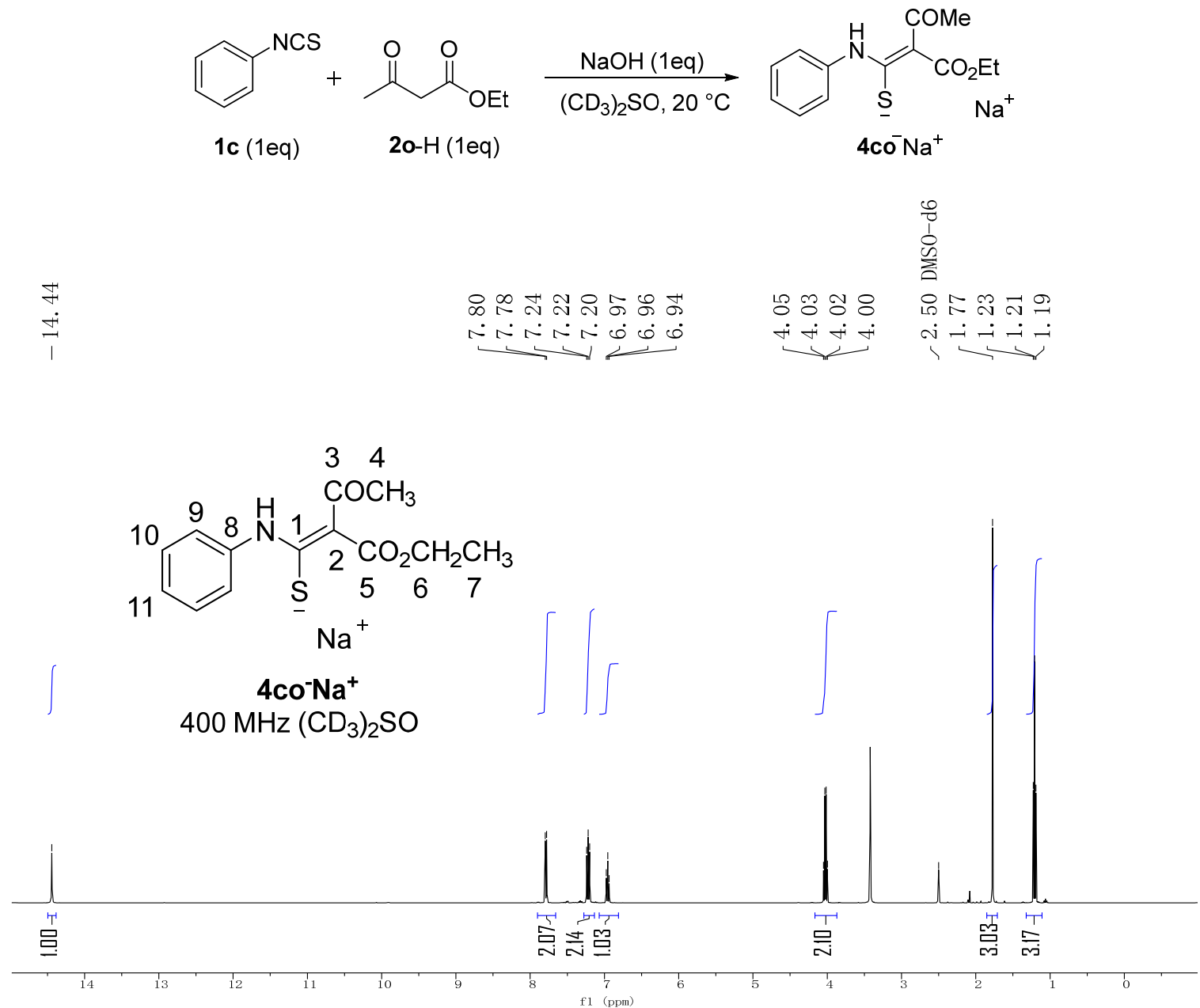

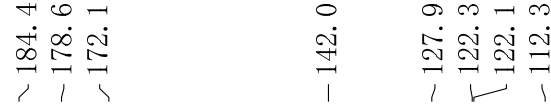
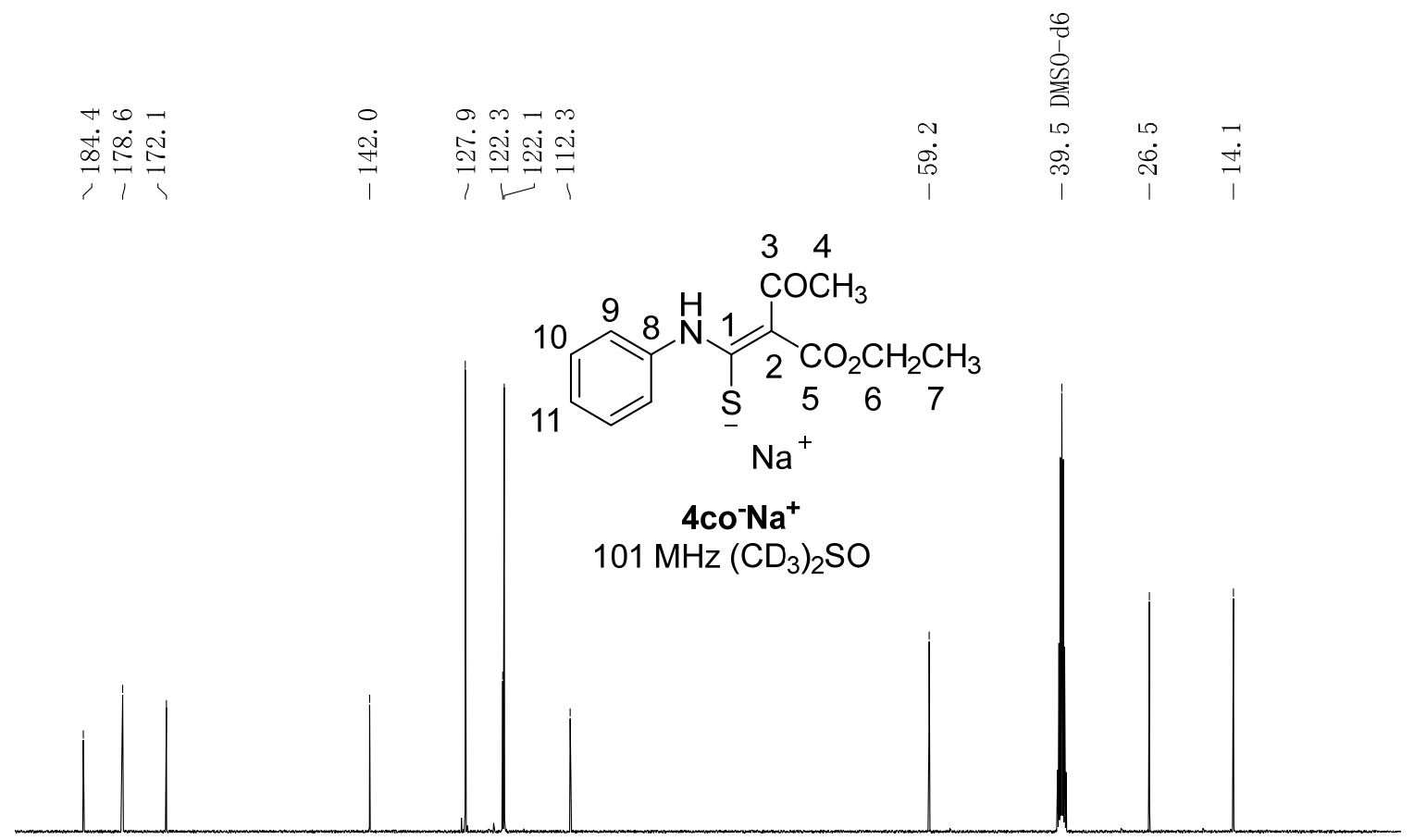
Crude reaction mixture: $\mathbf{1 c}+\mathbf{2 p}$ to give $4 \mathbf{c p} \mathrm{Na}^{+}$
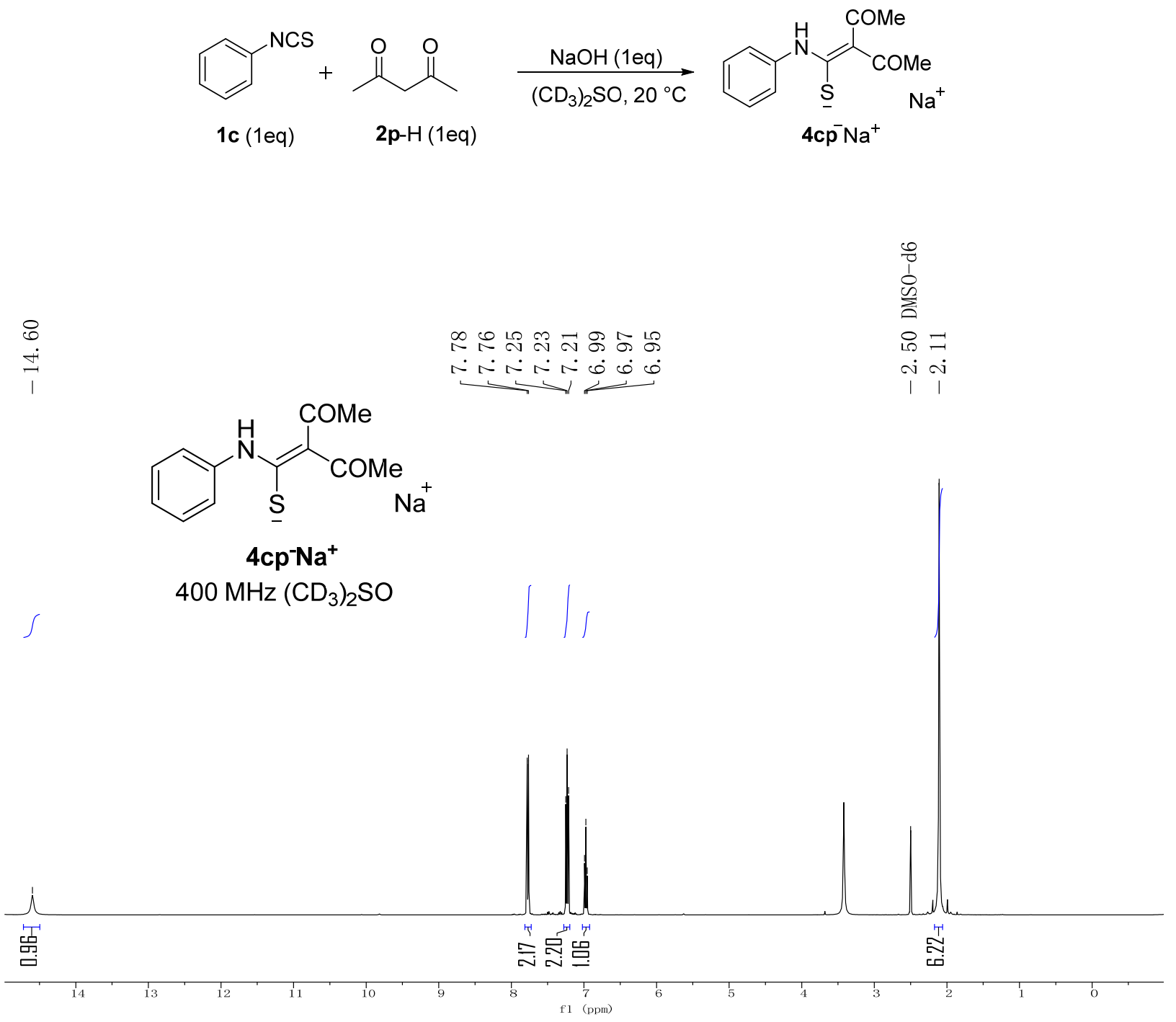
Crude reaction mixture: $\mathbf{1 d}+\mathbf{2 n}$ to give $\mathbf{4} \mathbf{d n}^{-} \mathrm{Na}^{+}$
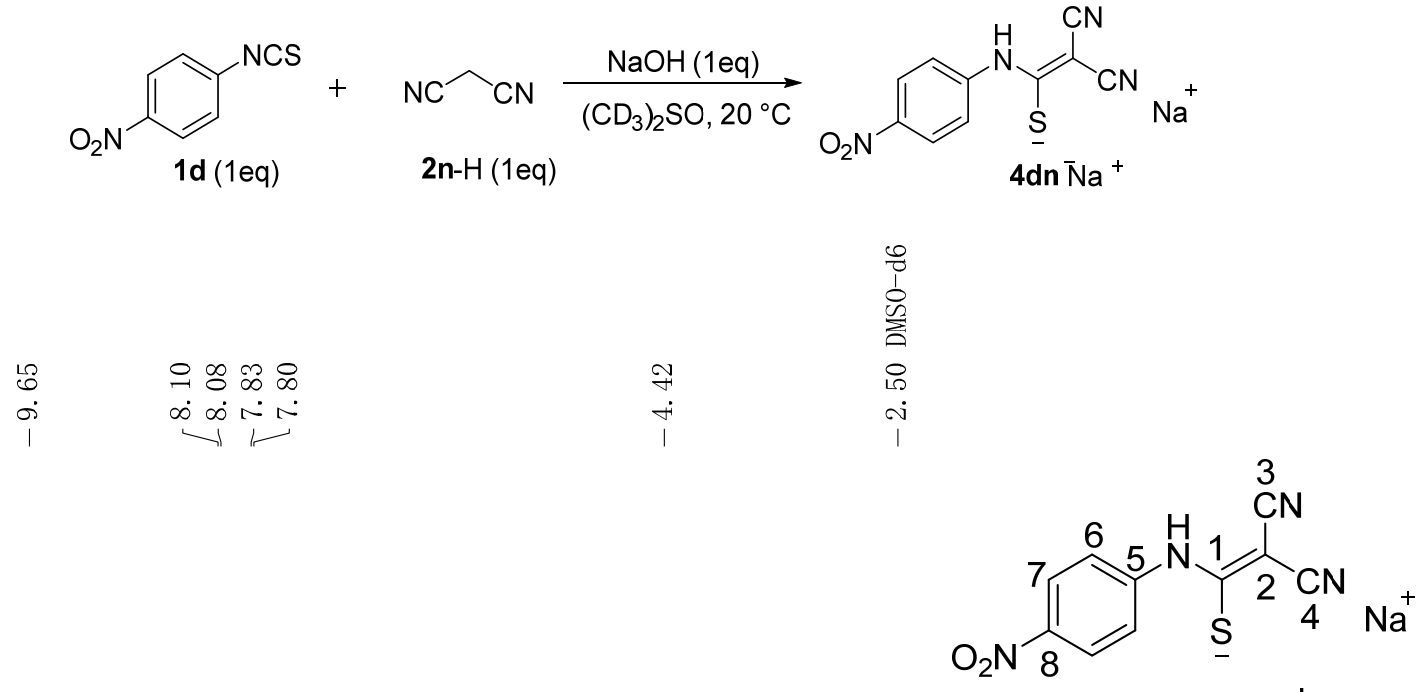

$4 \mathrm{dn}^{-} \mathrm{Na}^{+}$

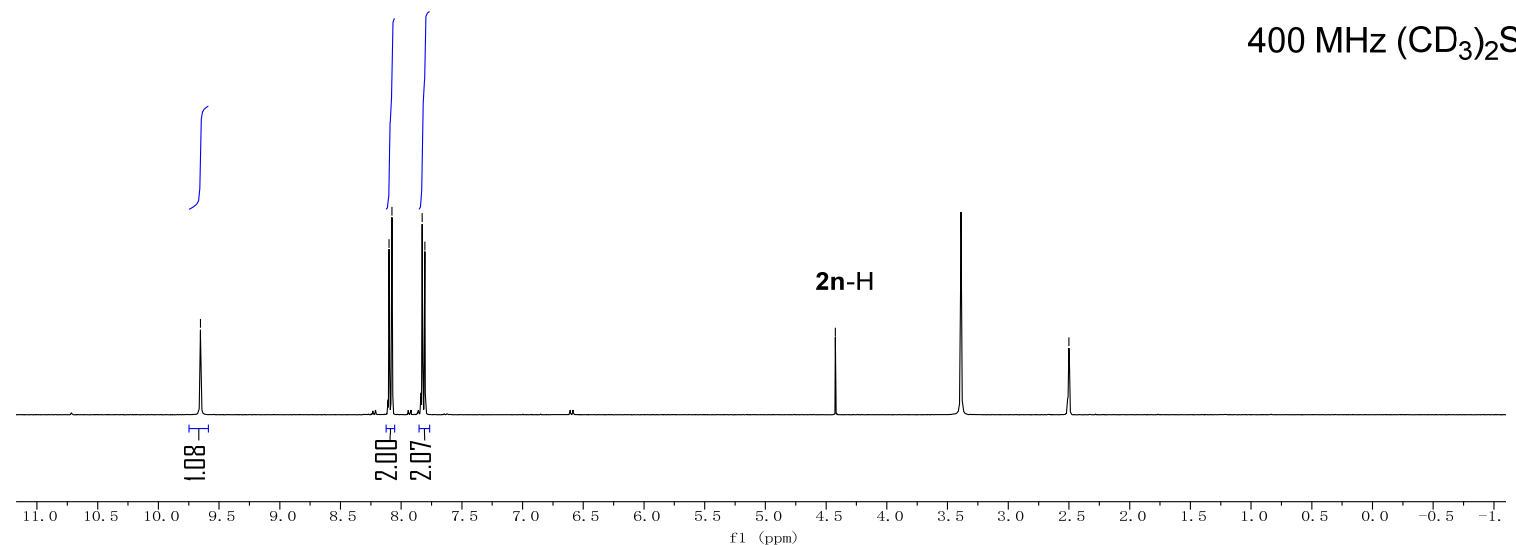

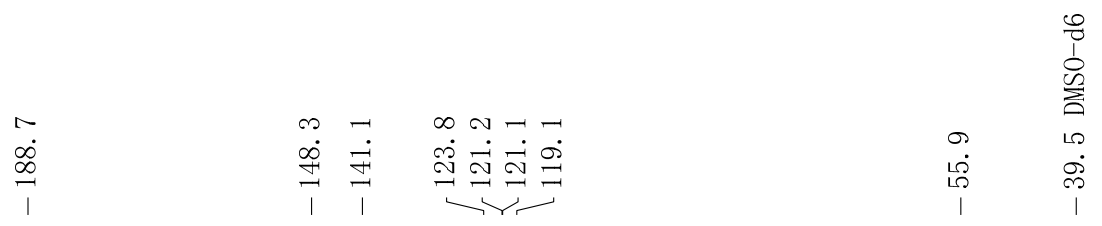

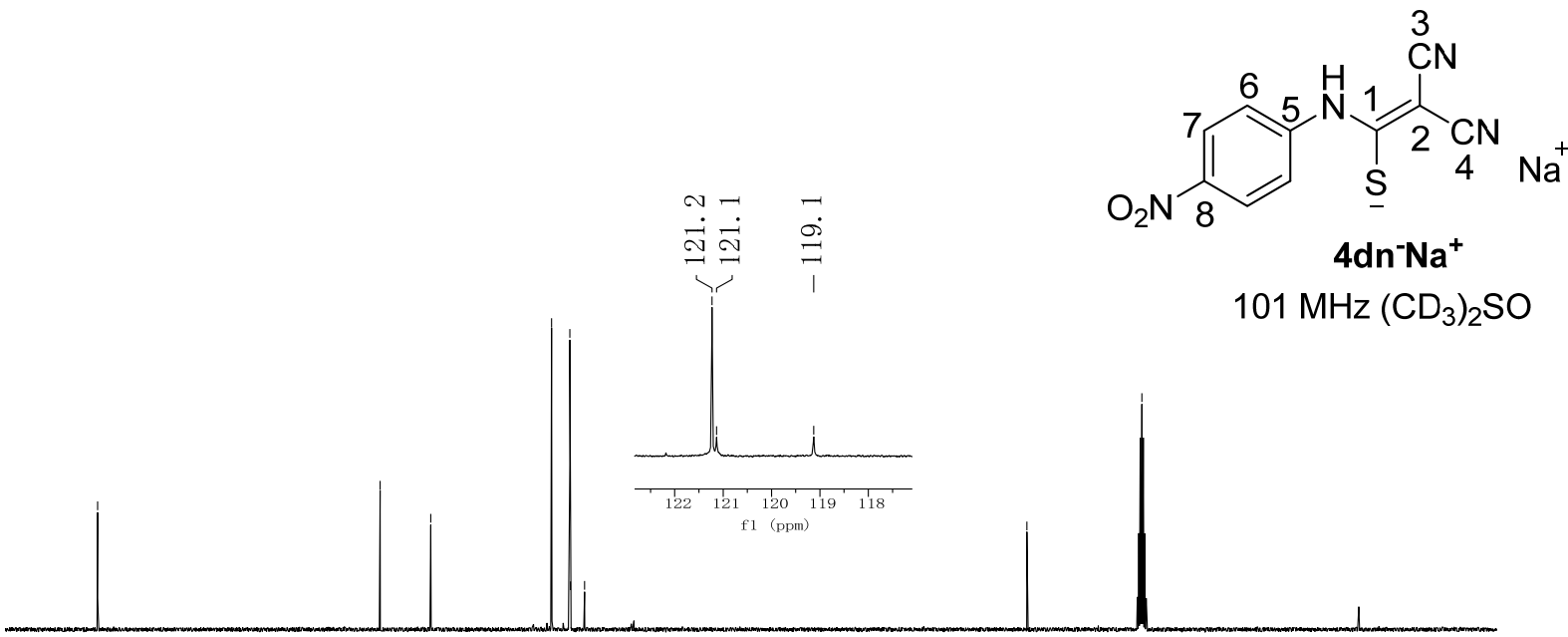

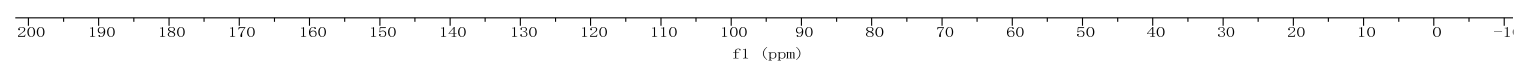


Crude reaction mixture: $\mathbf{1 d}+\mathbf{2 o}$ to give $\mathbf{4} \mathrm{do}^{-} \mathrm{Na}^{+}$

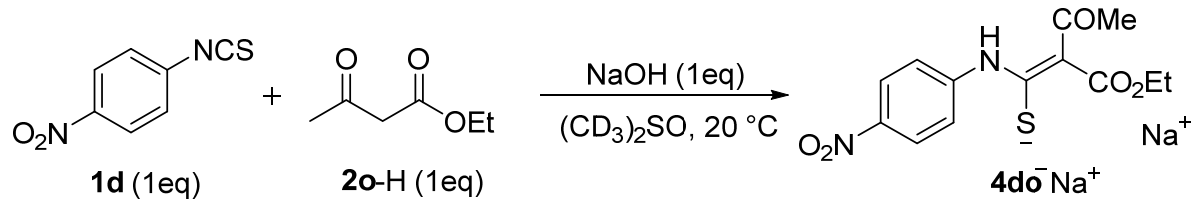
$\stackrel{8}{\stackrel{8}{\stackrel{10}{1}}}$

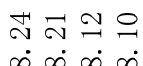
$\underbrace{\infty} \infty \infty^{\infty} \infty$

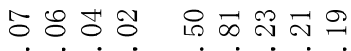

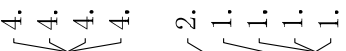

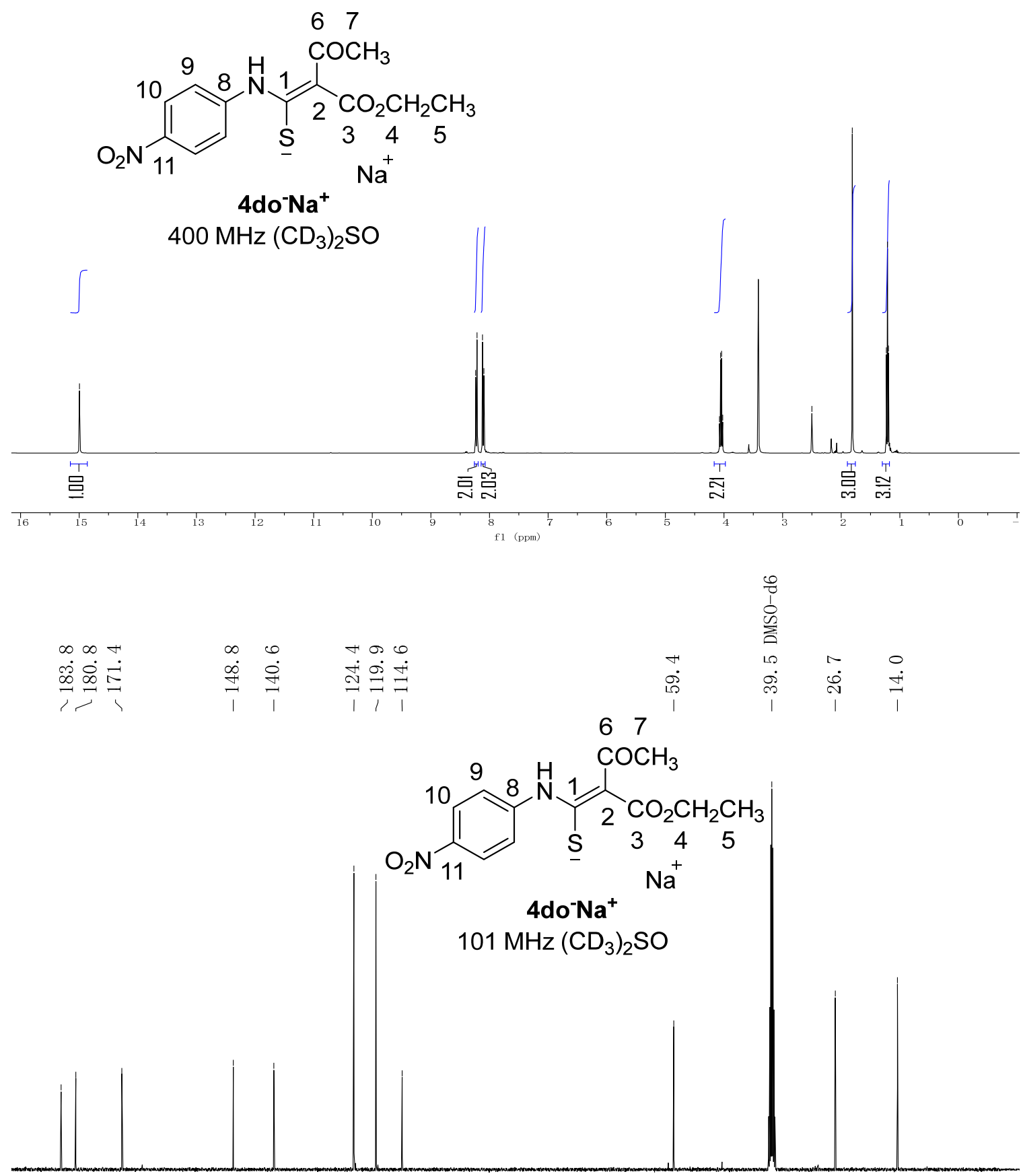

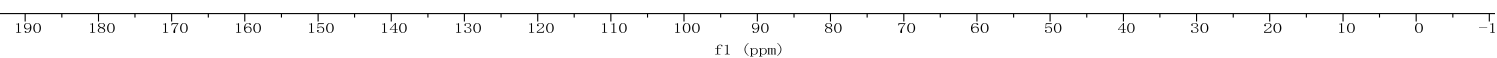




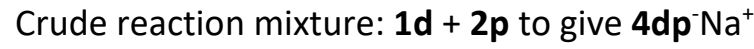<smiles>CC(=O)CC(C)=O</smiles><smiles></smiles>

$\exists$

$\stackrel{\text { 吕 }}{\text { | }}$
ลิ $\stackrel{2}{-1}$ 으

$$
\underbrace{\infty} \infty \infty^{\infty} \infty
$$

34<smiles>CC(=O)C(C(C)=O)=C(S)Nc1ccc([N+](=O)[O-])cc1</smiles>

4dp-Na+ $400 \mathrm{MHz}\left(\mathrm{CD}_{3}\right)_{2} \mathrm{SO}$<smiles>CC(=O)C(C(=[18O])S)=C(C)C</smiles>

$4 \mathrm{dp}^{-} \mathrm{Na}^{+}$

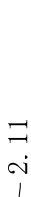

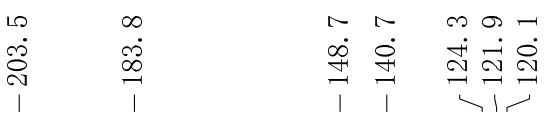

营
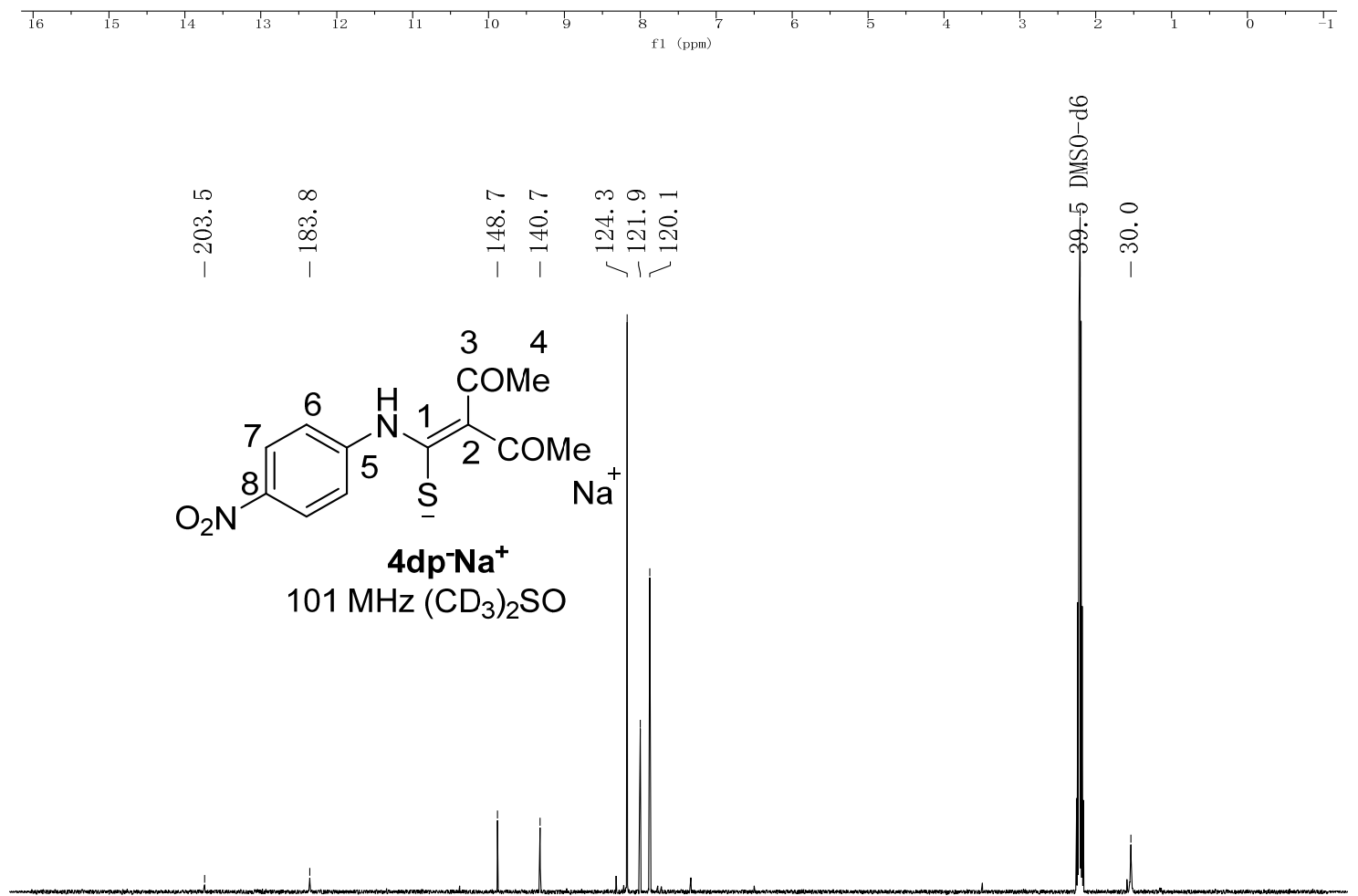

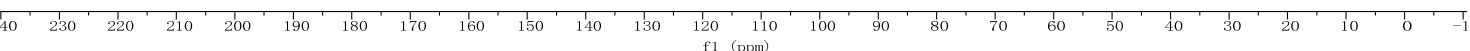




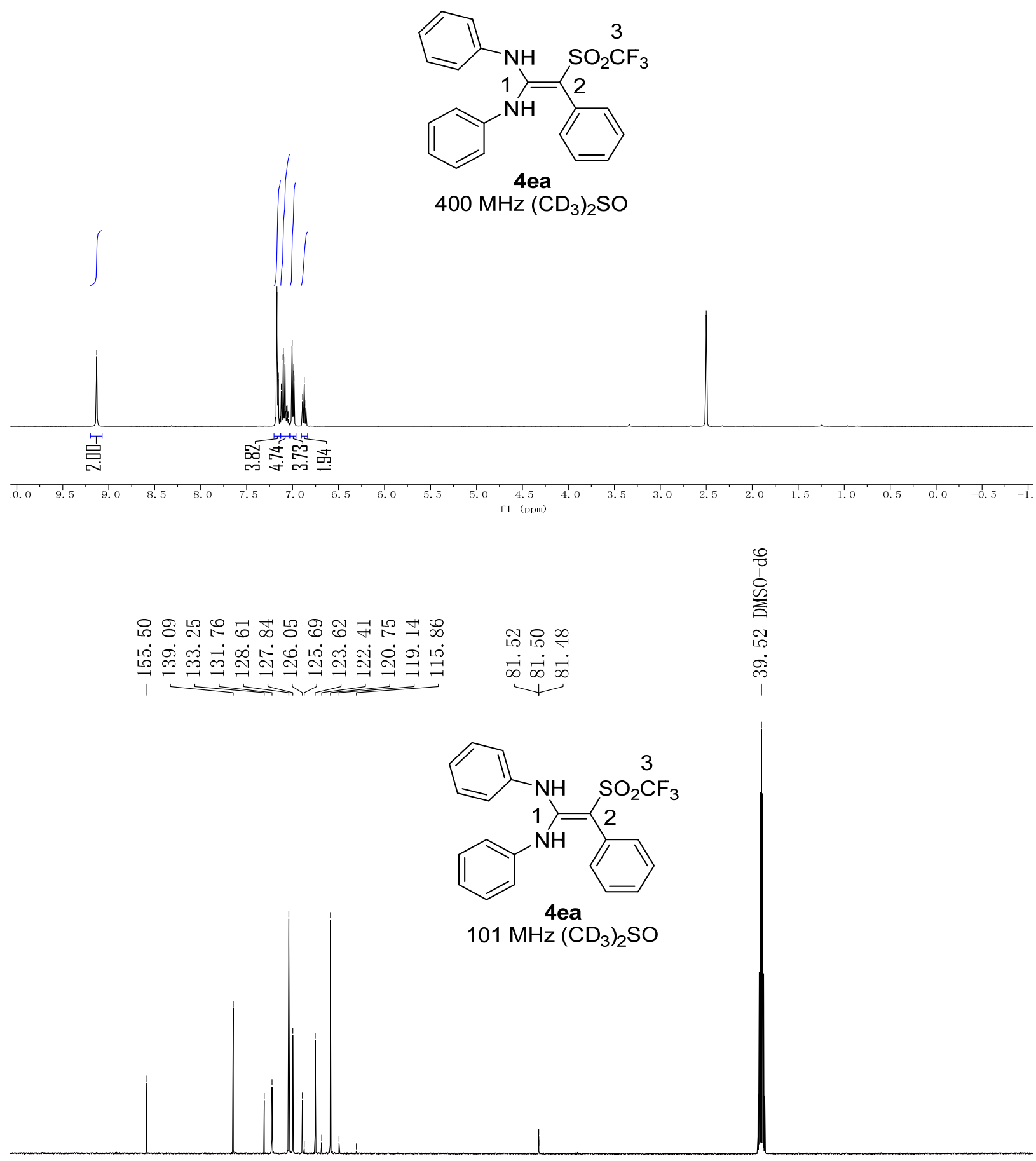

$\stackrel{7}{180}$

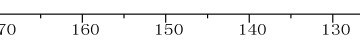

${ }_{120}^{1}+110$

f1 $\left(\begin{array}{cc}80 \\ \text { fpm) }\end{array}\right.$ 


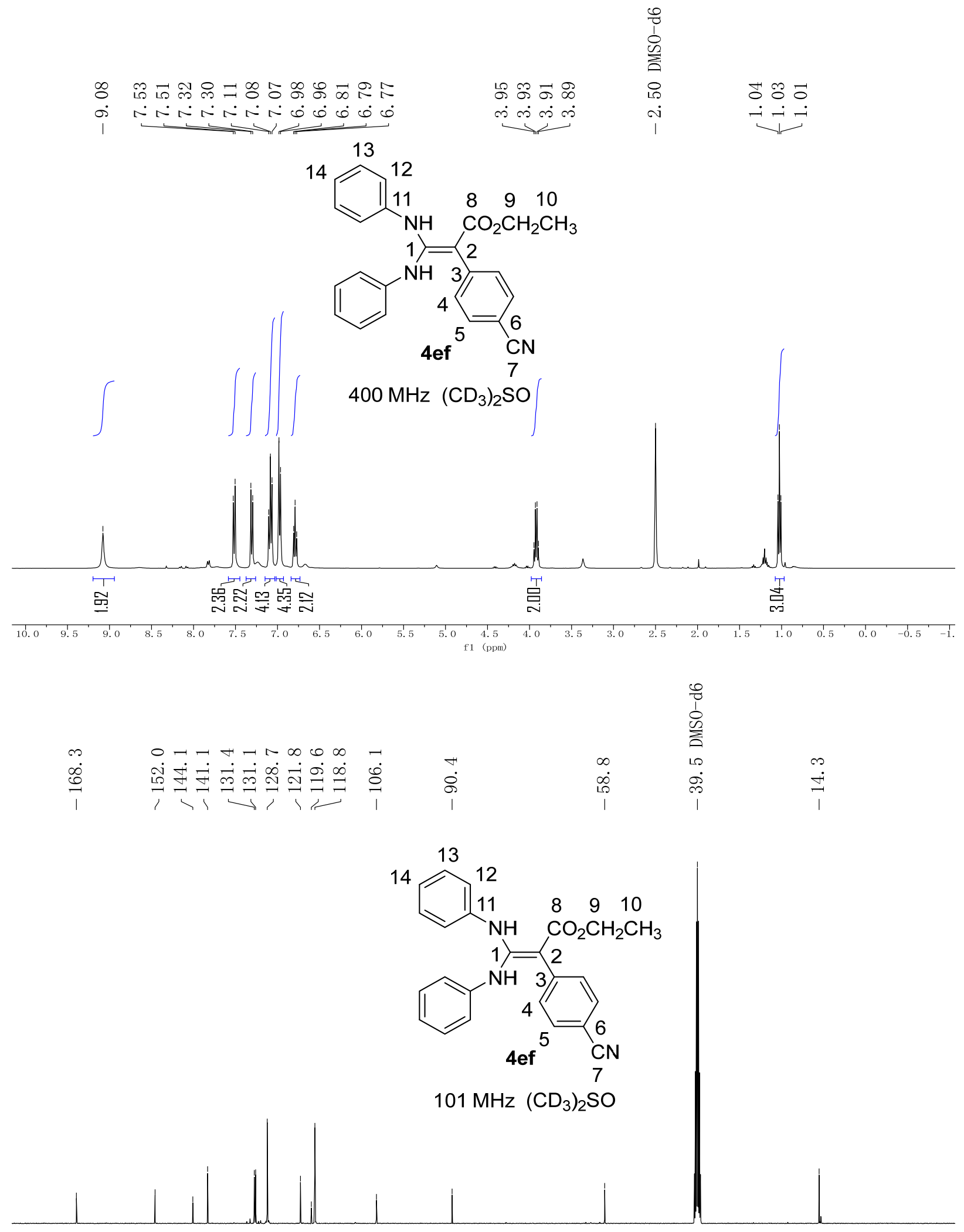




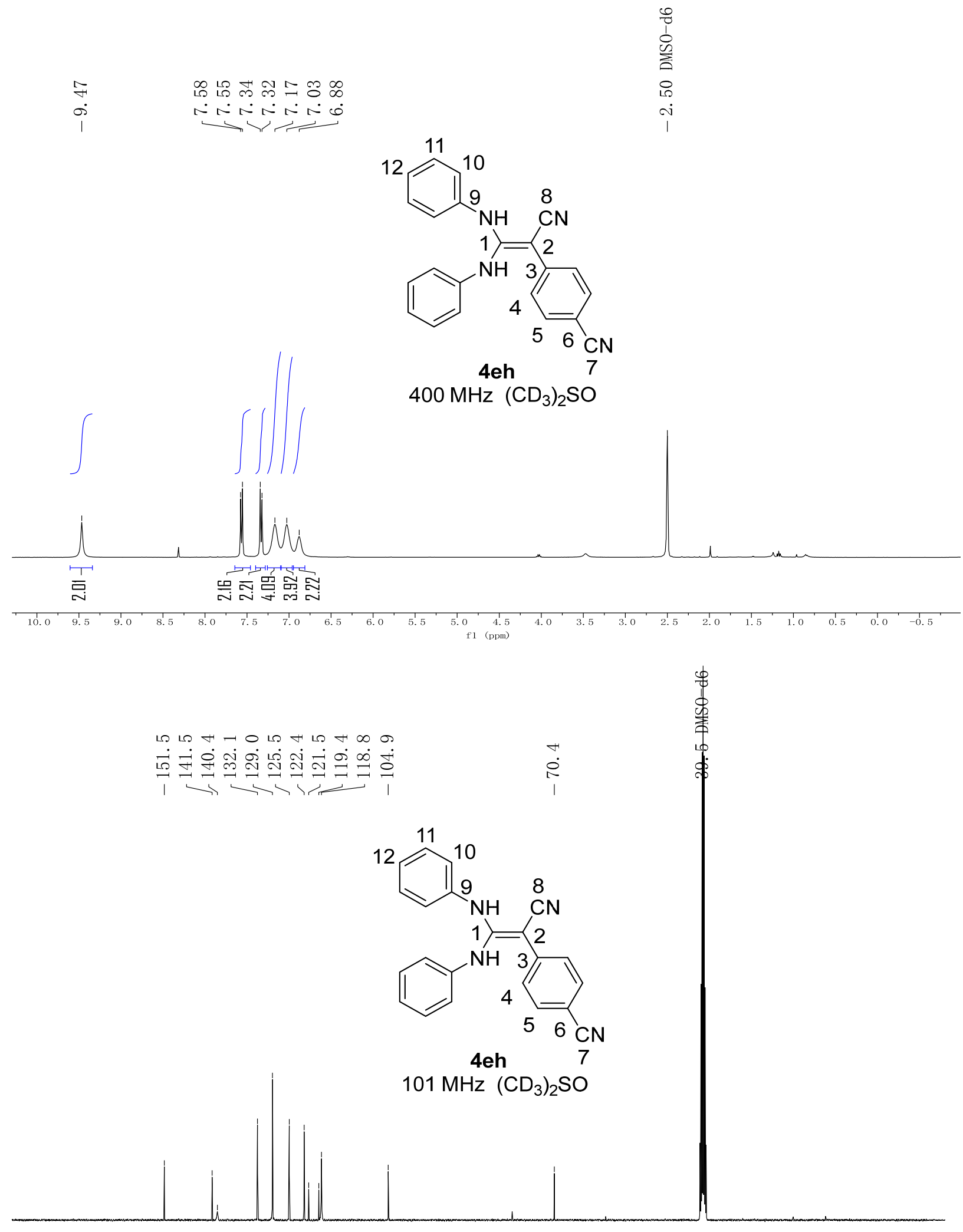

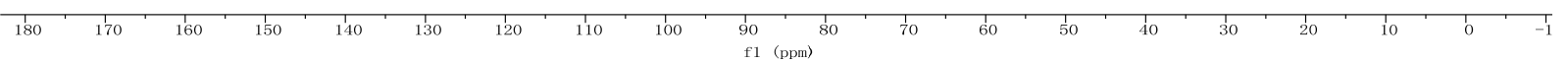




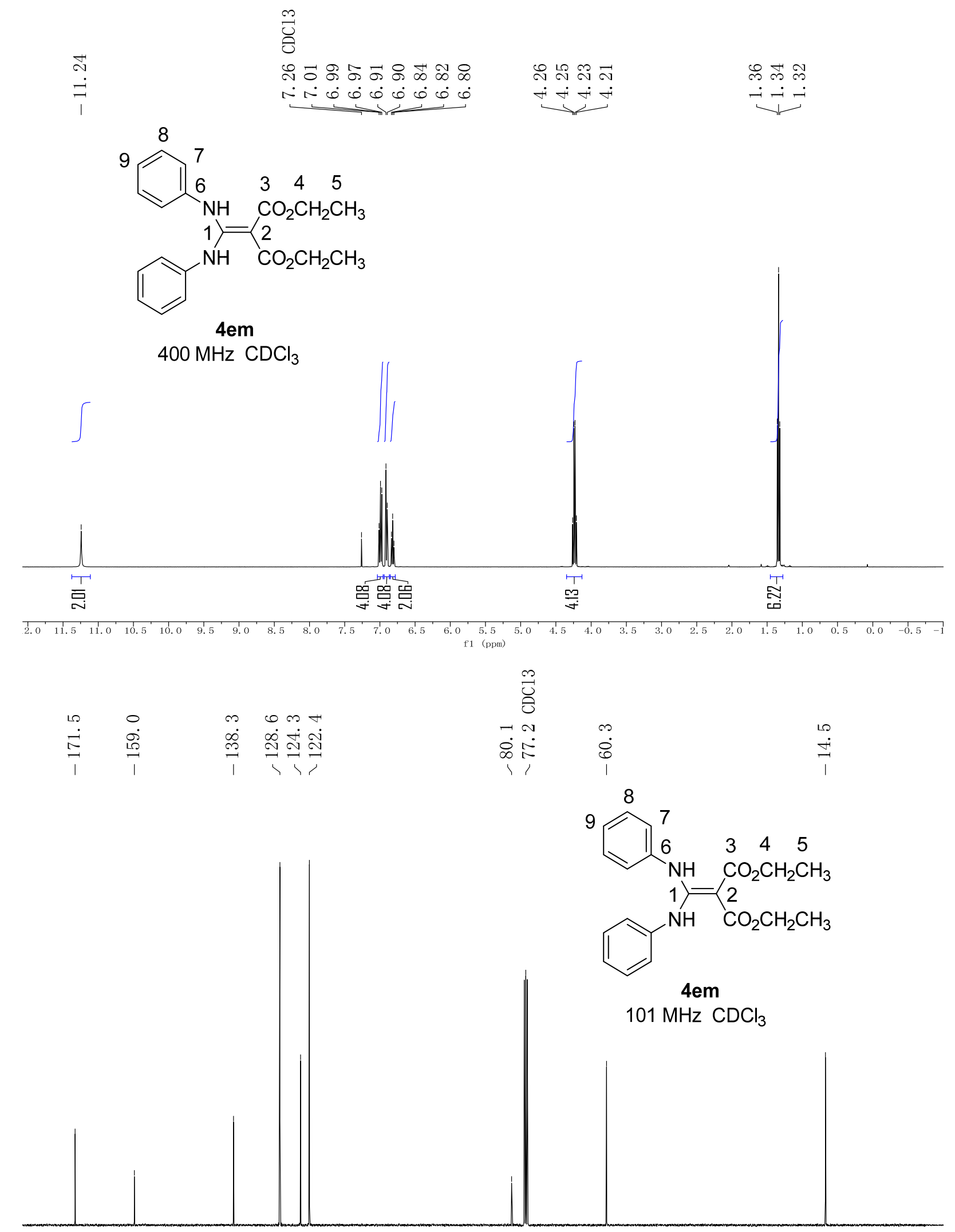

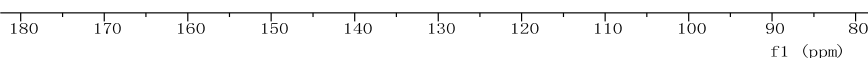

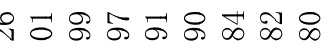

$\therefore$

ก ก

$\dot{-i} \dot{-}$

$\overbrace{}^{8}$

$\mathrm{NH} \quad \mathrm{CO}_{2} \mathrm{CH}_{2} \mathrm{CH}_{3}$

$\mathrm{NH} \quad \mathrm{CO}_{2} \mathrm{CH}_{2} \mathrm{CH}_{3}$

$4 \mathrm{em}$ 


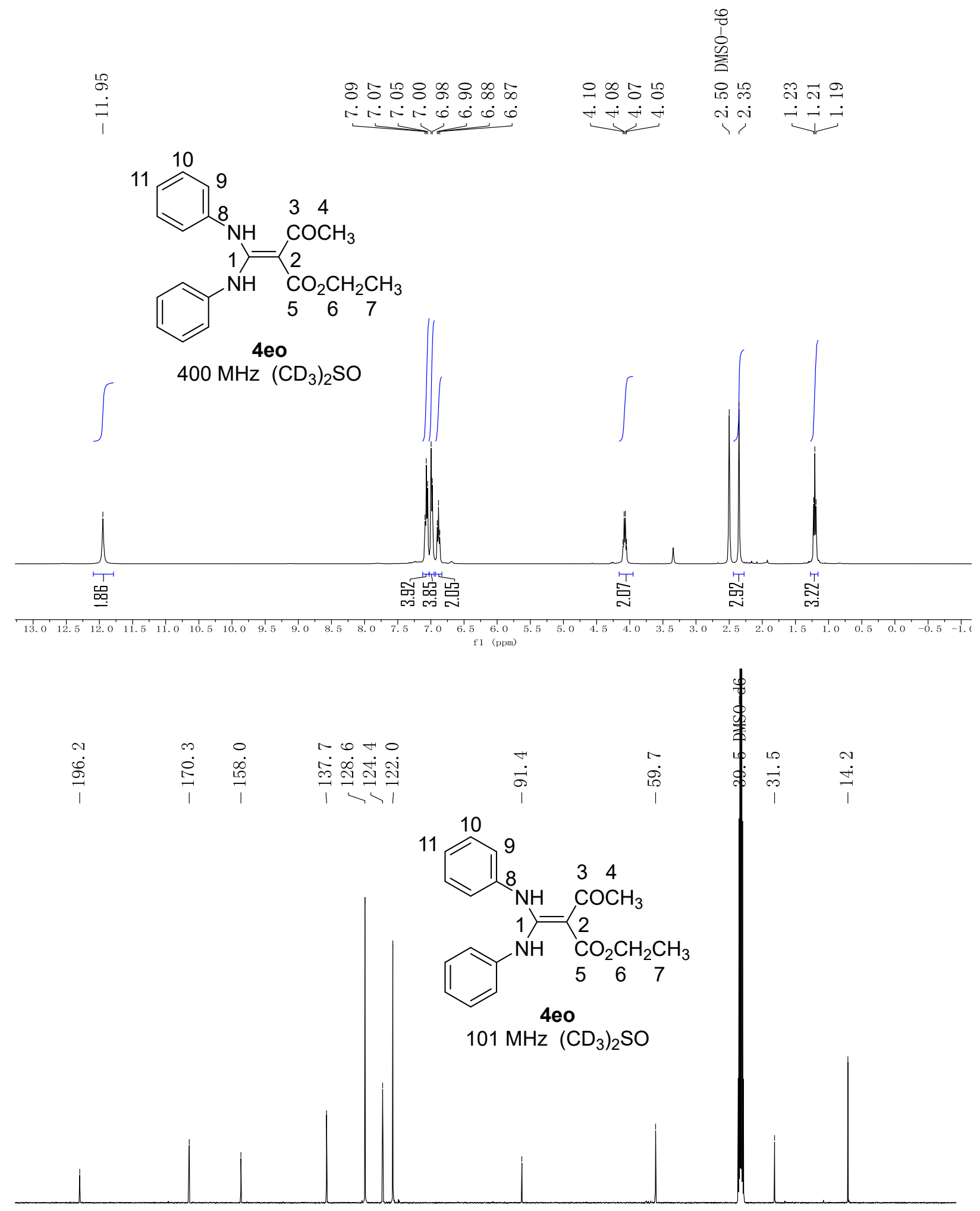

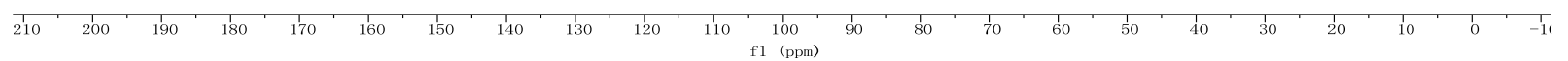


Crude reaction mixture: $\mathbf{1 g}+\mathbf{2 f}$ to give $\mathbf{4 g f} \mathrm{K}^{+}$
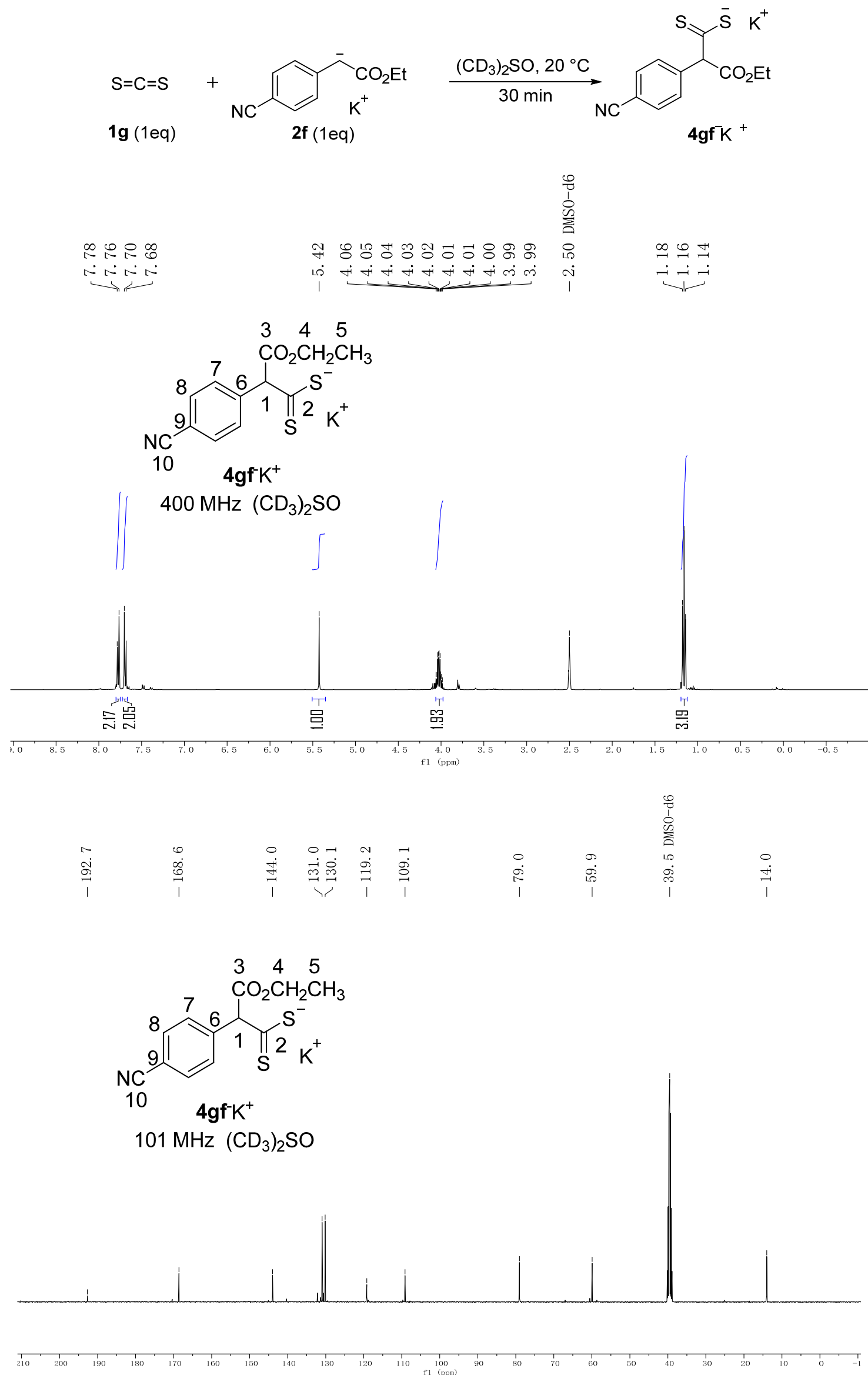


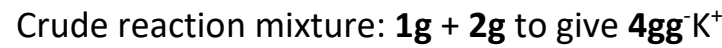

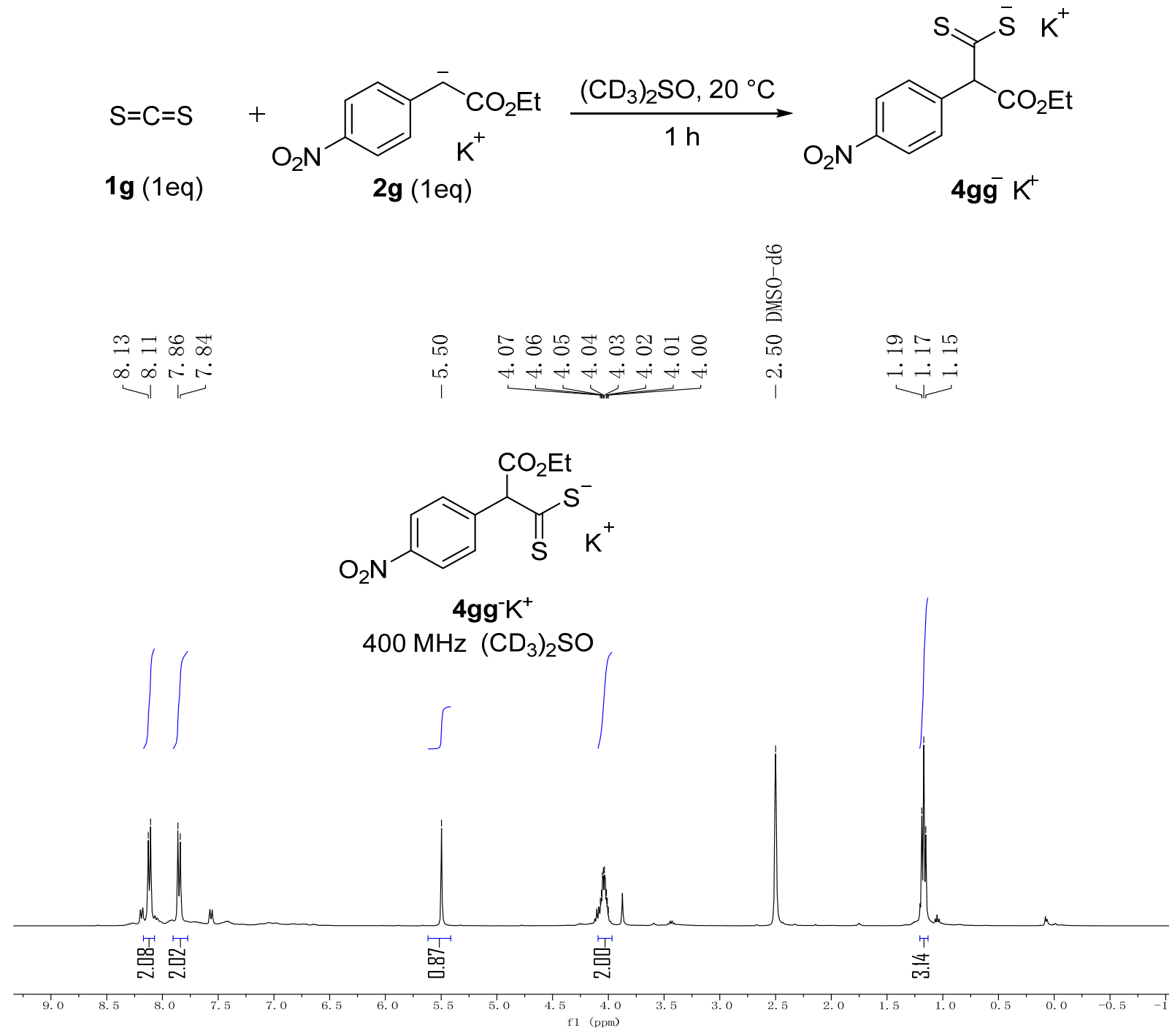


Crude reaction mixture: $\mathbf{1 g}+\mathbf{2} \mathbf{i}$ to give $\mathbf{4 g i} \mathrm{Na}^{+}$
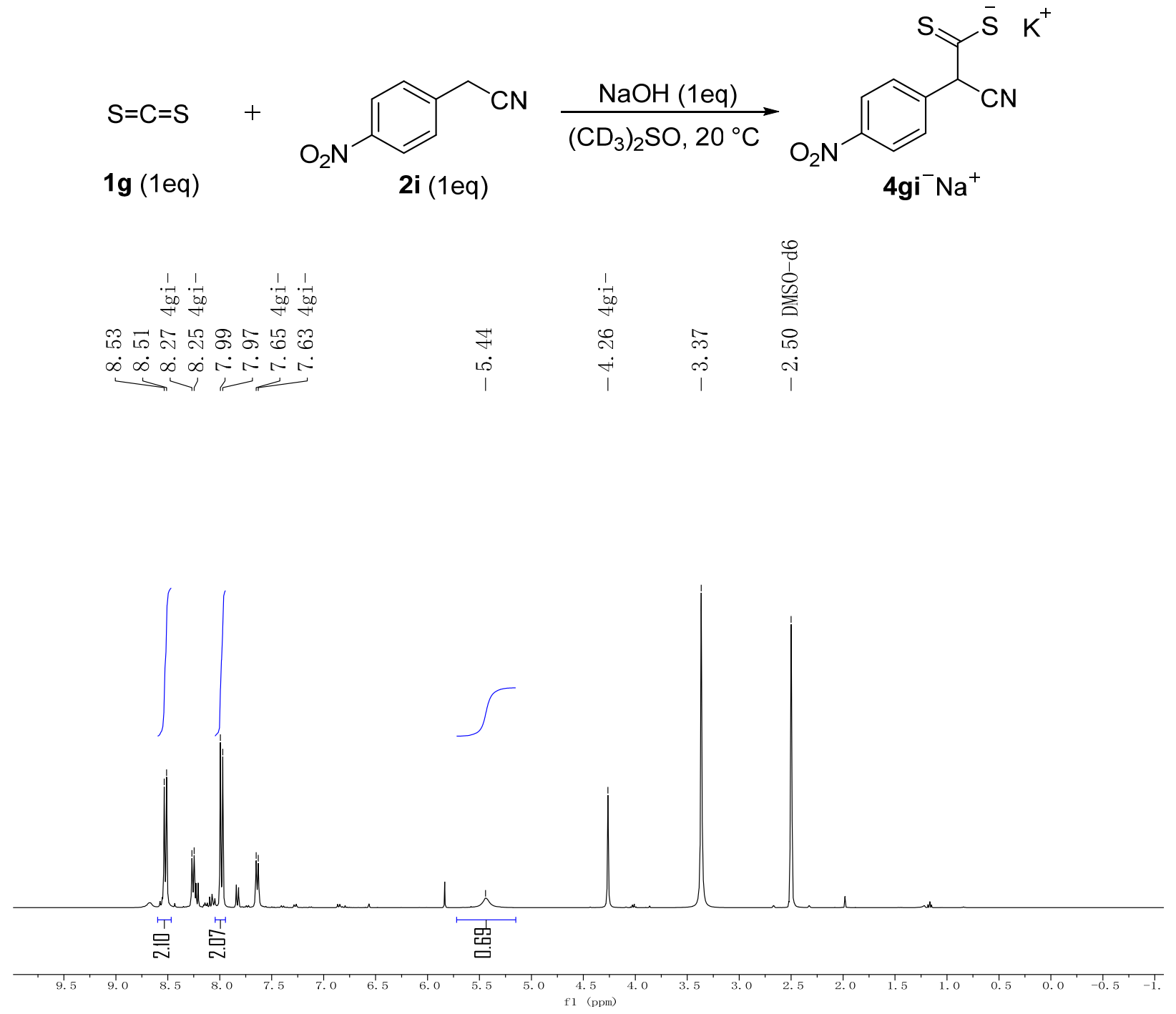

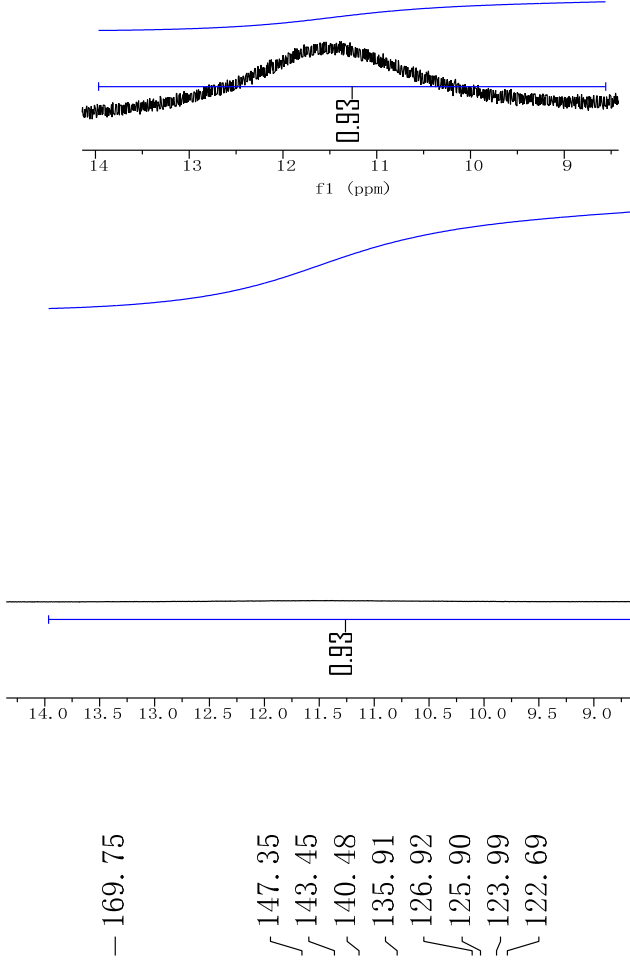

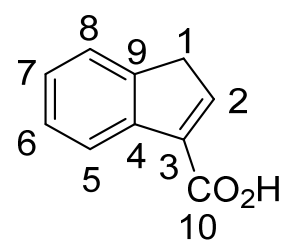

4hj

$400 \mathrm{MHz} \mathrm{CDCl}_{3}$

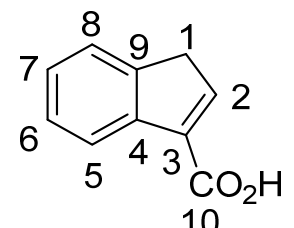

4hj

$101 \mathrm{MHz} \mathrm{CDCl}_{3}$
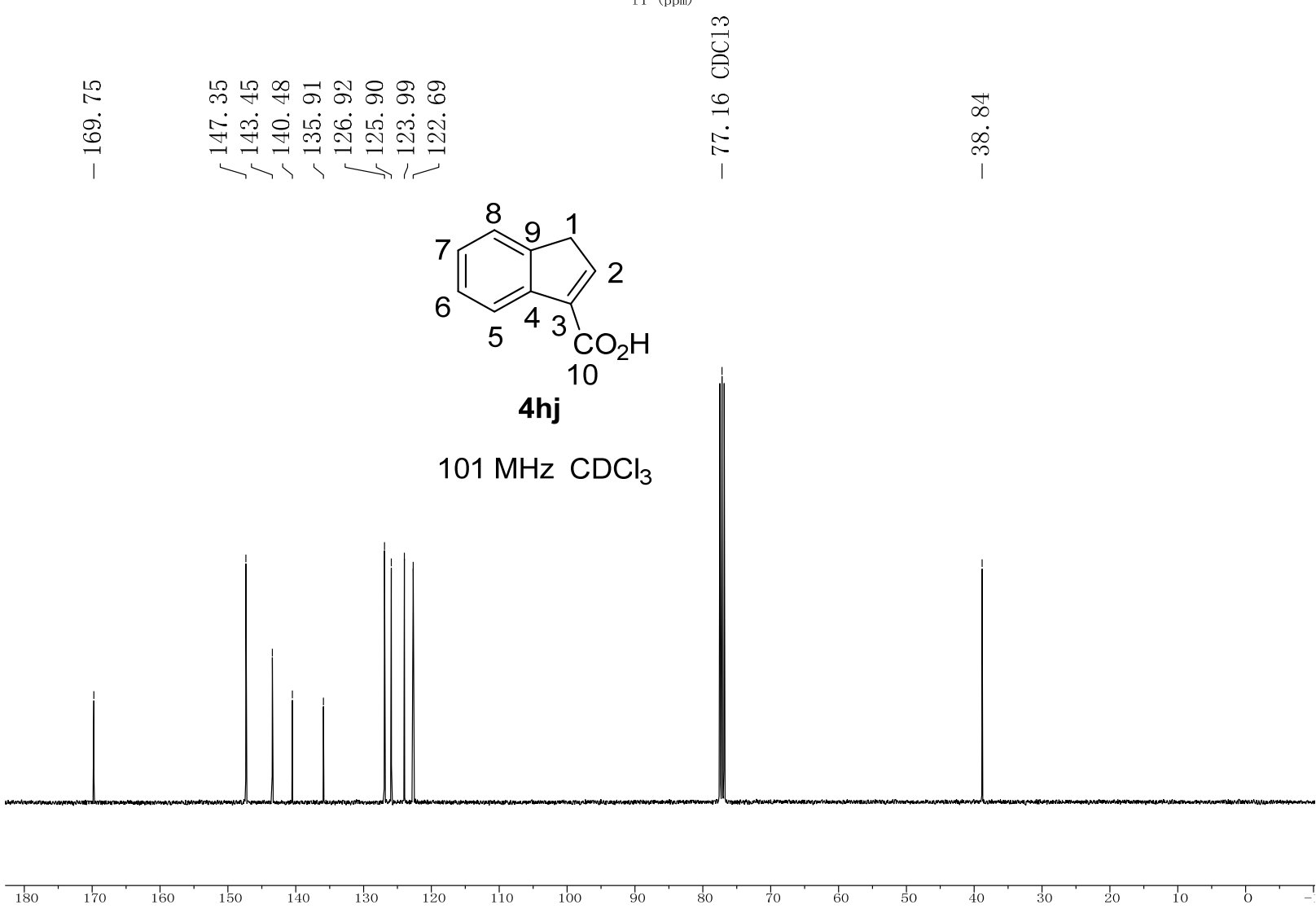


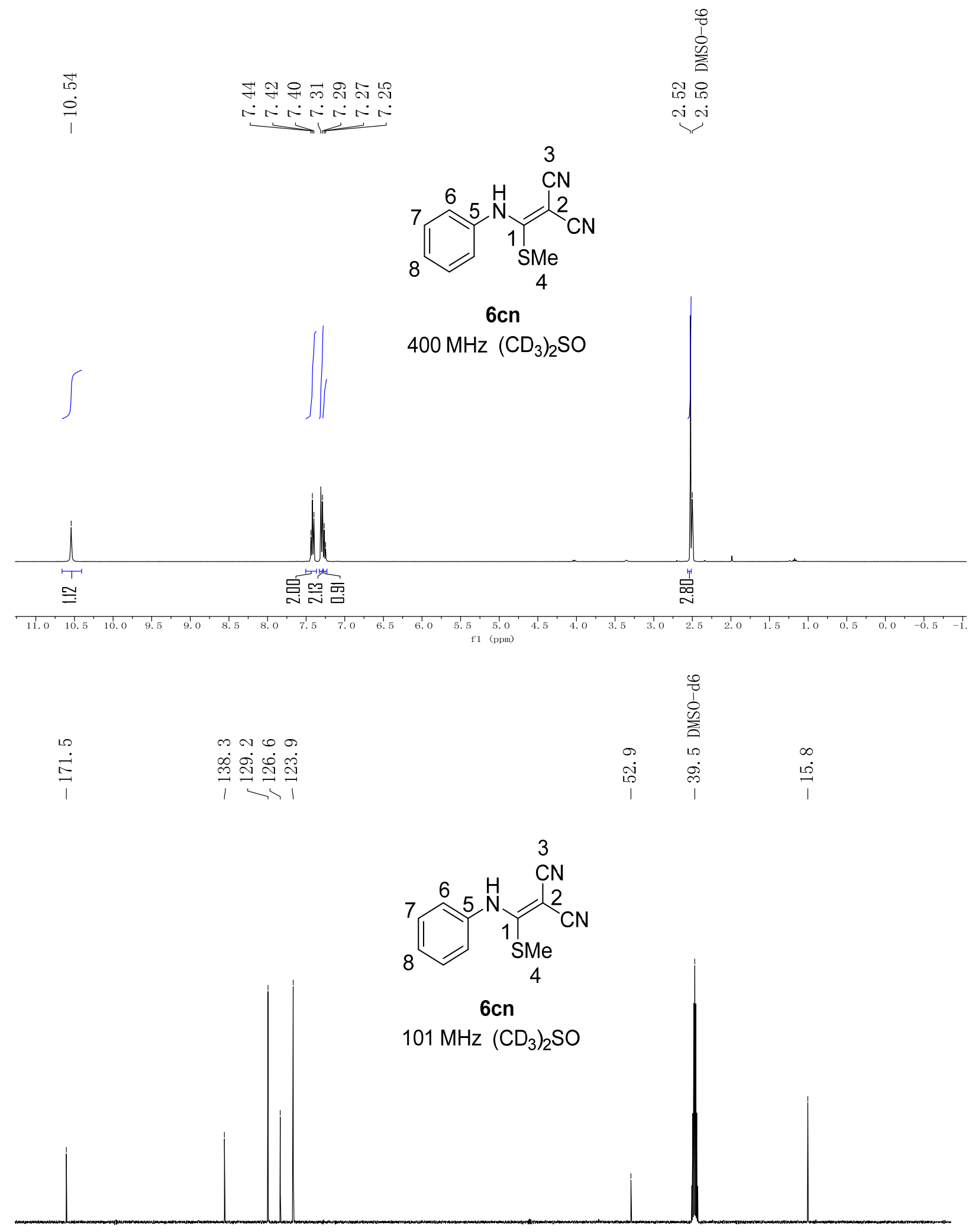

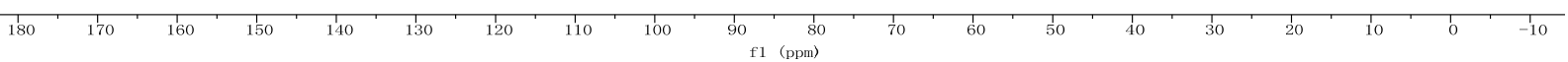




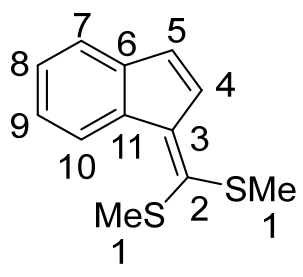

6gj

$400 \mathrm{MHz} \mathrm{CDCl}_{3}$

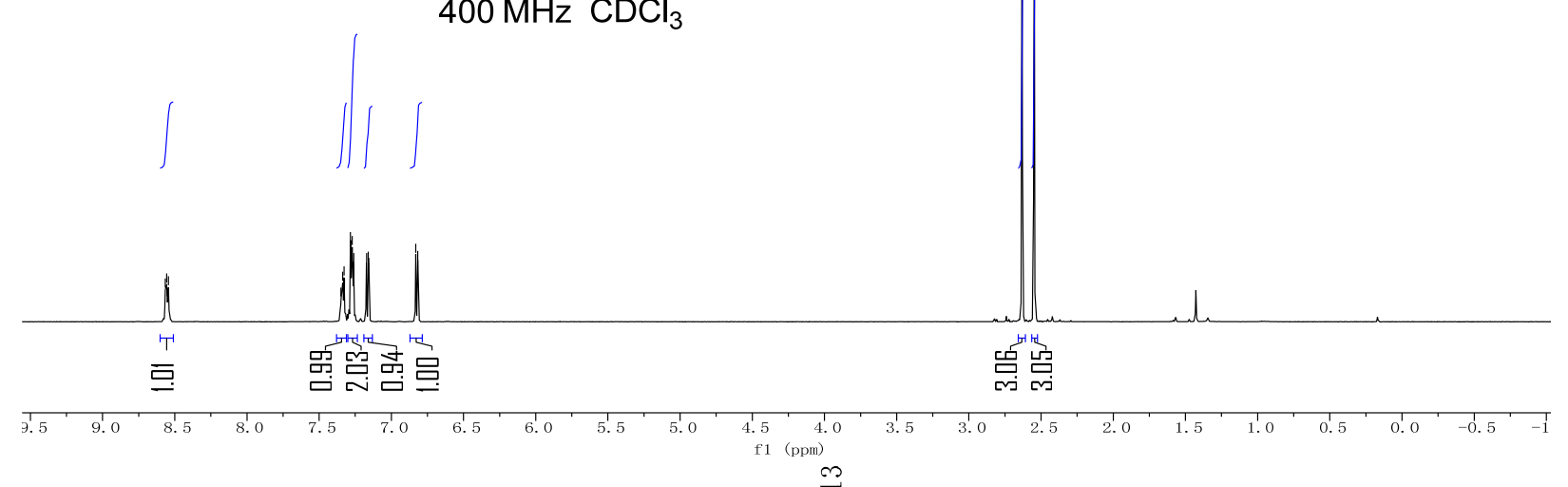

$\infty \forall m \sim a-\infty \forall \omega$

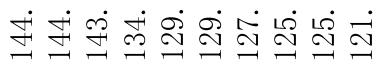
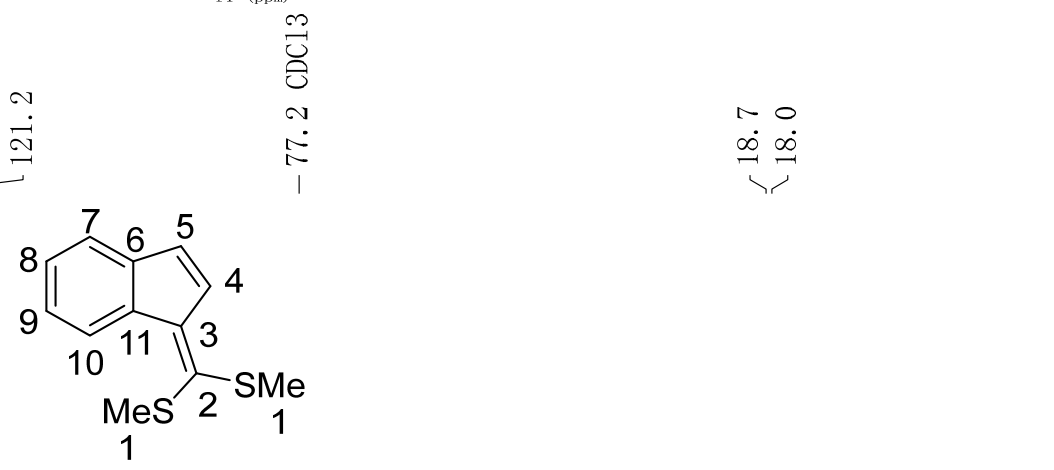

6gj
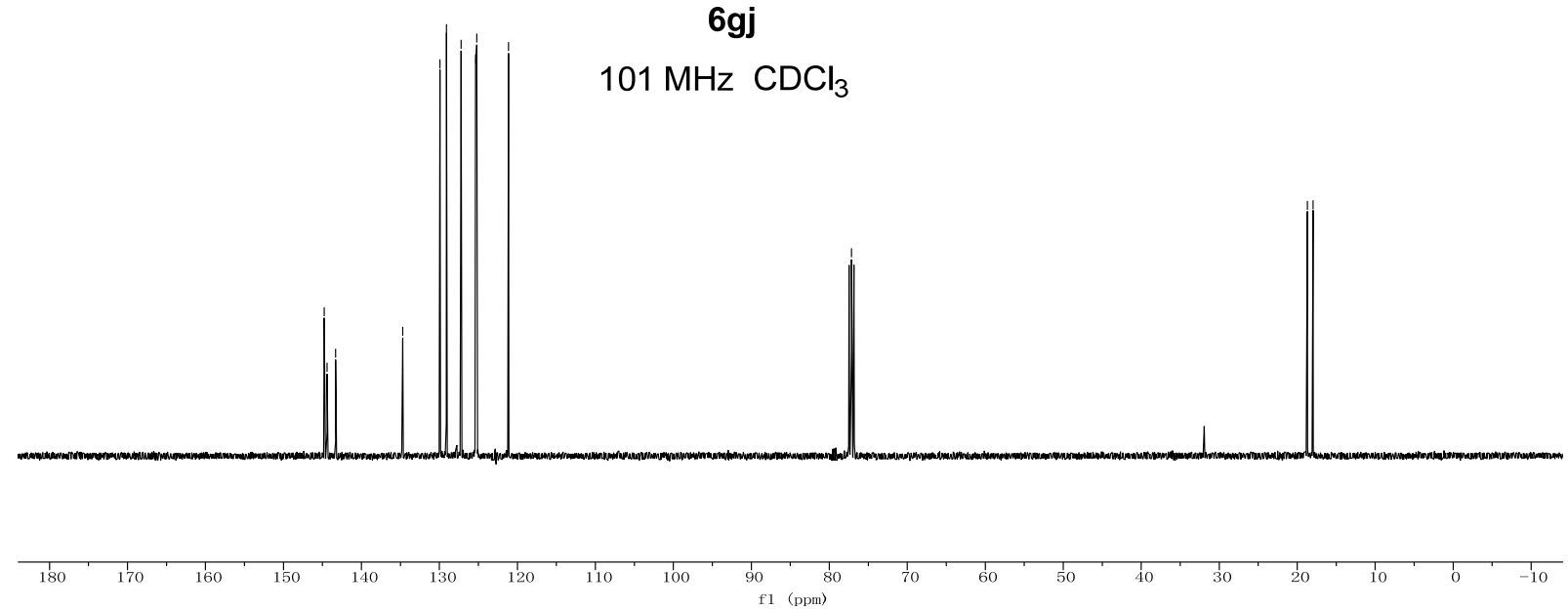
논

i i i

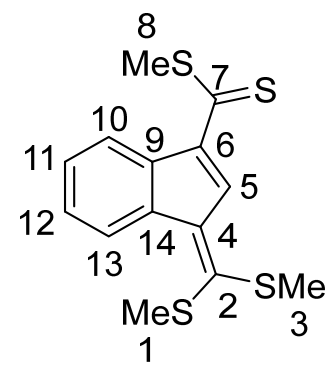

7gj

$400 \mathrm{MHz} \quad \mathrm{CDCl}_{3}$

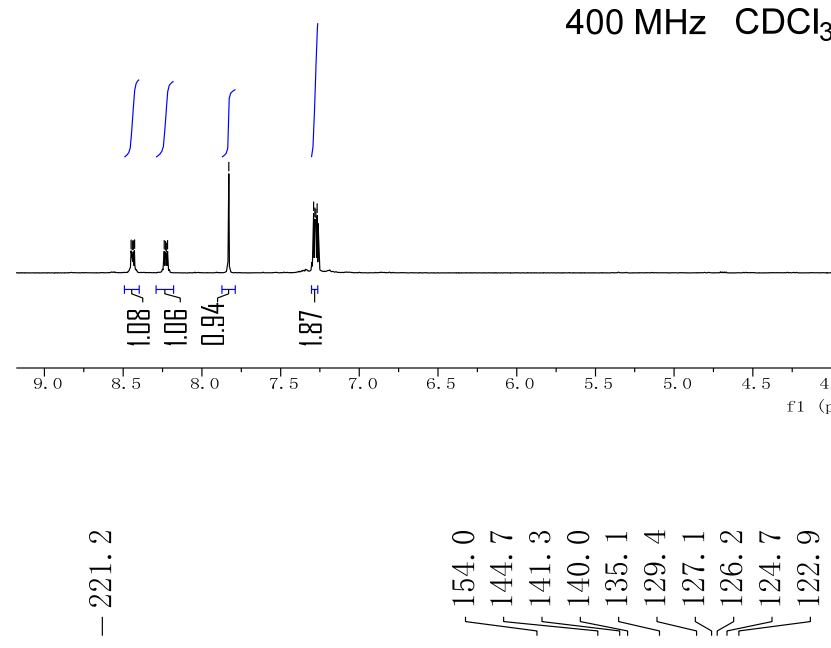

by

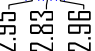

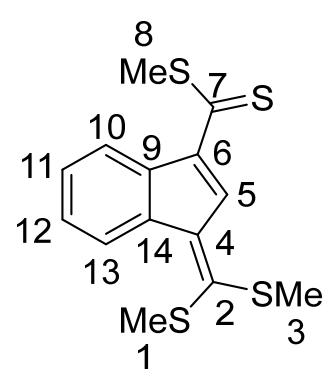

7gj

$101 \mathrm{MHz} \quad \mathrm{CDCl}_{3}$
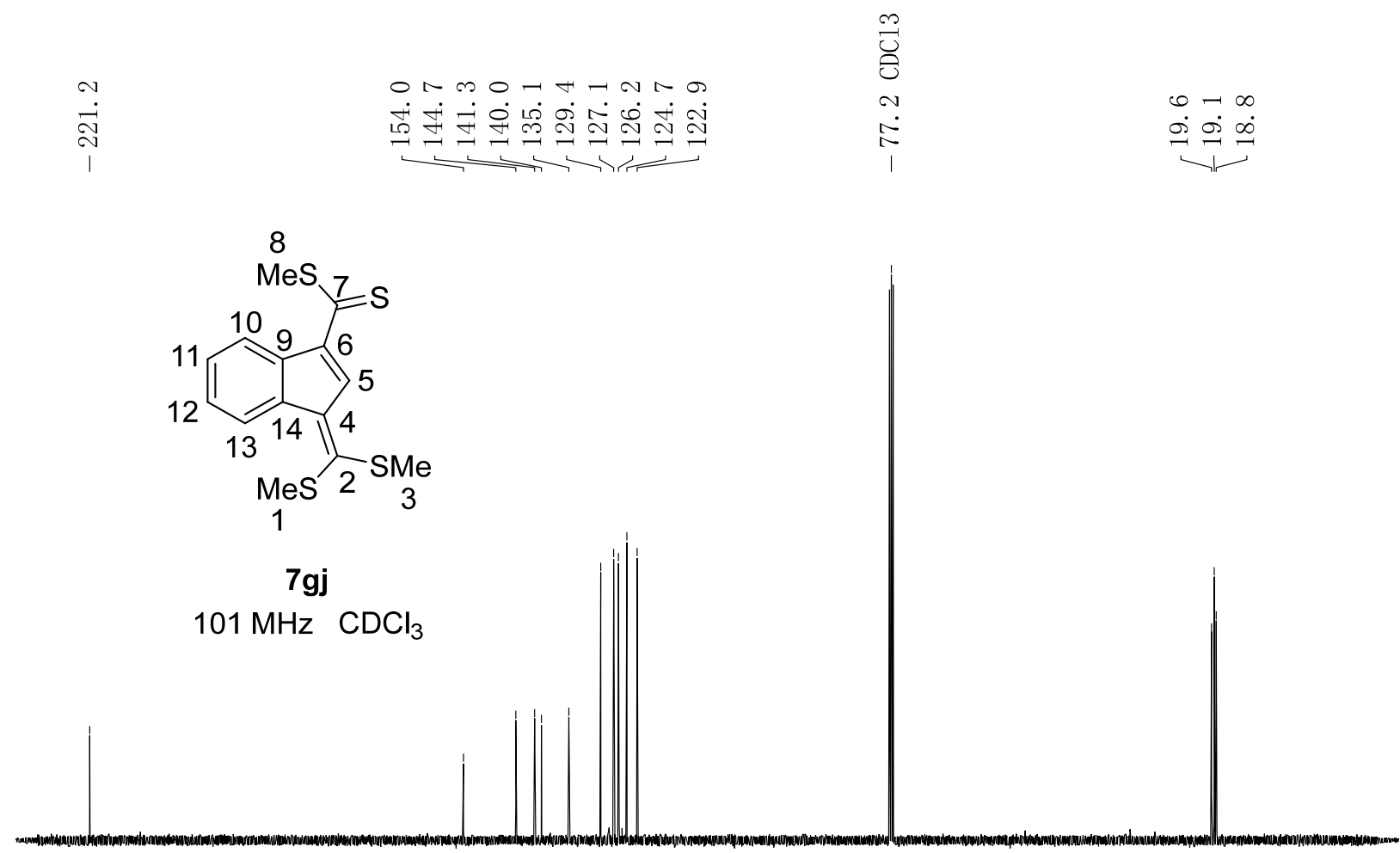


\section{References}

(S1) Fulmer, G. R.; Miller, A. J. M.; Sherden, N. H.; Gottlieb, H. E.; Nudelman, A.; Stoltz, B. M.; Bercaw, J. E.; Goldberg, K. I. Organometallics 2010, 29, 2176-2179.

(S2) Ali, A. R.; Ghosh, H.; Patel, B. K. Tetrahedron Lett. 2010, 51, 1019-1021.

(S3) Zhang, M.; Badal, M. M. R.; Koppel, I. A.; Mishima, M. Bull. Chem. Soc. Jpn. 2013, $86,813-820$.

(S4) Scott, J. P.; Hammond, D. C.; Beck, E. M.; Brands, K. M. J.; Davies, A. J.; Dolling, U. H.; Kennedy, D. J. Tetrahedron Lett. 2004, 45, 3345-3348.

(S5) Timofeeva, D. S.; Mayer, R.; Mayer, P.; Ofial, A. R.; Mayr, H. Chem. Eur. J. 2018, 24, $5901-5910$.

(S6) Tacke, M.; Allen, T.; Cuffe, L.; Gallagher, W. M.; Lou, Y.; Mendoza, O.; Müller-Bunz, H.; Rehmann, F.-J. K.; Sweeney, N. J. Organomet. Chem. 2004, 689, 2242-2249.

(S7) Schembri, L. S.; Eriksson, J.; Odell, L. R. J. Org. Chem. 2019, 84, 6970-6981.

(S8) (a) Effenberger, F.; Gleiter, R. Angew. Chem. 1963, 75, 450-451. (b) Effenberger, F.; Gleiter, R. Chem. Ber. 1964, 97, 1576-1583.

(S9) Sommen, G.; Comel, A.; Kirsch, G. Tetrahedron 2003, 59, 1557-1564.

(S 10 ) (a) Resch, S.: Schneider, A.-R.; Beichler, R.; Spera, M. B. M.; Fanous, J.; Schollmeyer, D.; Waldvogel, S. R. Eur. J. Org. Chem. 2015, 933-937. (b) Traube, W.; Eyme, A. Chem. Ber. 1899, 32, 3176-3178.

(S11) Dorokhov, V. A.; Komkov, A. V. Russ. Chem. Bull., Int. Ed. 2004, 53, 676-680.

(S12) Millar, R. W.; Neuenschwander, M. Chimia 1979, 33, 54-56.

(S13) Pietruszka, J.; Simon, R. C.; Kruska, F.; Braun, M. Eur. J. Org. Chem. 2009, 62176224.

(S14) For the reactivity parameters $N$ and $s \mathrm{~N}$ of the carbanions in DMSO, see the database at www.cup.lmu.de/oc/mayr/DBintro.html.

(S15) (a) Bordwell, F. G. Acc. Chem. Res. 1988, 21, 456-463. (b) Bordwell, F. G.; Fried, H. E. J. Org. Chem. 1981, 46, 4327-4331. (c) Bordwell, F. G.; Cheng, J-P.; Bausch, M. J. Bares, J. E. J. Phys. Org. Chem. 1988, 1, 209-223. (d) Keeffe, J. R.; Palmer, C. A.; Lee, J. C. J. Am. Chem. Soc. 1979, 101, 1295-1297. (e) Bordwell, F. G.; Harrelson, J. A. Can. J. Chem. 1990, 68, 1714-1718. (f) Matthews, W. S.; Bares, J. E.; Bartmess, J. E.; Bordwell, F. G.; Cornforth, F. J.; Drucker, G. E.; Margolin, Z.; McCallum, R. J.; McCollum, G. J.; Vanier, N. R. J. Am. Chem. Soc. 1975, 97, 7006-7014. (g) Olmstead, W. N.; Bordwell, F. G. J. Org. Chem. 1980, 45, 3299-3305. (h) Petrov, E. S.; Tsvetkov, E. N.; Mesyats, S. P.; Shatenshtein, A. N. Kabachnik, M. I. Russ. Chem. Bull. 1976, 25, 762-766. (i) Bordwell, F. G.; Drucker, G. E. J. Org. Chem. 1980, 45, 3325-3328.

(S16) (a) Mayr, H.; Bug, T.; Gotta, M. F.; Hering, N.; Irrgang, B.; Janker, B.; Kempf, B.; Loos, R.; Ofial, A. R.; Remennikov, G.; Schimmel, H. J. Am. Chem. Soc. 2001, 123, 9500-9512. (b) Richter, D.; Hampel, N.; Singer, T.; Ofial, A. R.; Mayr, H. Eur. J. Org. Chem. 2009, 3203-3211. (c) Lucius, R.; Loos, R.; Mayr, H. Angew. Chem., Int. Ed. 2002, 41, 91-95.

(S17) Hua, L. Phys. Chem. Liq. 2009, 47, 296-301. 
(S18) Harder, E.; Damm, W.; Maple, J.; Wu, C.; Reboul, M.; Xiang, J. Y.; Wang, L.; Lupyan, D.; Dahlgren, M. K.; Knight, J.L.; Kaus, J. W.; Cerutti, D. S.; Krilov, G.; Jorgensen, W. L.; Abel, R.; Friesner, R. A. J. Chem. Theory Comput. 2016, 12, 281-296. 2019.

(S19) Schrödinger Release 2019-1: MacroModel, Schrödinger, LLC, New York, NY,

(S20) Gaussian 16, Revision A.03, Frisch, M. J.; Trucks, G. W.; Schlegel, H. B.; Scuseria, G. E.; Robb, M. A.; Cheeseman, J. R.; Scalmani, G.; Barone, V.; Petersson, G. A.; Nakatsuji, H.; Li, X.; Caricato, M.; Marenich, A. V.; Bloino, J.; Janesko, B. G.; Gomperts, R.; Mennucci, B.; Hratchian, H. P.; Ortiz, J. V.; Izmaylov, A. F.; Sonnenberg, J. L.; Williams-Young, D.; Ding, F.; Lipparini, F.; Egidi, F.; Goings, J.; Peng, B.; Petrone, A.; Henderson, T.; Ranasinghe, D.; Zakrzewski, V. G.; Gao, J.; Rega, N.; Zheng, G.; Liang, W.; Hada, M.; Ehara, M.; Toyota, K.; Fukuda, R.; Hasegawa, J.; Ishida, M.; Nakajima, T.; Honda, Y.; Kitao, O.; Nakai, H.; Vreven, T.; Throssell, K.; Montgomery, J. A., Jr.; Peralta, J. E.; Ogliaro, F.; Bearpark, M. J.; Heyd, J. J.; Brothers, E. N.; Kudin, K. N.; Staroverov, V. N.; Keith, T. A.; Kobayashi, R.; Normand, J.; Raghavachari, K.; Rendell, A. P.; Burant, J. C.; Iyengar, S. S.; Tomasi, J.; Cossi, M.; Millam, J. M.; Klene, M.; Adamo, C.; Cammi, R.; Ochterski, J. W.; Martin, R. L.; Morokuma, K.; Farkas, O.; Foresman, J. B.; Fox, D. J. Gaussian, Inc., Wallingford CT, 2016.

(S21) (a) Allgäuer, D. S.; Jangra, H.; Asahara, H.; Li, Z.; Chen, Q.; Zipse, H.; Ofial, A. R.; Mayr, H. J. Am. Chem. Soc. 2017, 139, 13318-13329. (b) Li, Z.; Jangra, H.; Chen, Q.; Mayer, P.; Ofial, A. R.; Zipse, H.; Mayr, H. J. Am. Chem. Soc. 2018, 140, 5500-5515.

(S22) Marenich, A. V.; Cramer, C. J.; Truhlar, D. G. J. Phys. Chem. B 2009, 113, 63786396.

(S23) Yang, W.; Mortier, W. J. J. Am. Chem. Soc. 1986, 108, 5708-5711.

(S24) Becke, A. D. J. Chem. Phys. 1993, 98, 5648-5652.

(S25) Cances, E.; Mennucci, B.; Tomasi, J. J. Chem. Phys. 1997, 107, 3032-3041.

(S26) Grimme, S.; Antony, J., Ehrlich, S., Krieg, H. J. Chem. Phys. 2010, 132, 154104.

(S27) Svatunek, D.; Houk, K. N. J. Comput. Chem. 2019, 40, 2509-2515.

(S28) Bickelhaupt, F. M.; Houk, K. N. Angew. Chem. Int. Ed. 2017, 56, 10070-10086. 